Florida International University

FIU Digital Commons

FIU Electronic Theses and Dissertations

University Graduate School

$11-2-2015$

\title{
Prediction of Fracture Toughness and Durability of Adhesively Bonded Composite Joints with Undesirable Bonding Conditions
}

Vishal Musaramthota

Florida International University, vmusa001@fiu.edu

DOI: $10.25148 /$ etd.FIDC000242

Follow this and additional works at: https://digitalcommons.fiu.edu/etd

Part of the Materials Science and Engineering Commons

\section{Recommended Citation}

Musaramthota, Vishal, "Prediction of Fracture Toughness and Durability of Adhesively Bonded Composite Joints with Undesirable Bonding Conditions" (2015). FIU Electronic Theses and Dissertations. 2513.

https://digitalcommons.fiu.edu/etd/2513

This work is brought to you for free and open access by the University Graduate School at FIU Digital Commons. It has been accepted for inclusion in FIU Electronic Theses and Dissertations by an authorized administrator of FIU Digital Commons. For more information, please contact dcc@fiu.edu. 


\title{
FLORIDA INTERNATIONAL UNIVERSITY
}

Miami, Florida

PREDICTION OF FRACTURE TOUGHNESS AND DURABILITY OF ADHESIVELY BONDED COMPOSITE JOINTS WITH UNDESIRABLE BONDING CONDITIONS

\author{
A dissertation submitted in partial fulfillment of the \\ requirements for the degree of \\ DOCTOR OF PHILOSOPHY \\ in \\ MATERIAL SCIENCE AND ENGINEERING \\ by \\ Vishal Musaramthota
}


To: Interim Dean Ranu Jung

College of Engineering and Computing

This dissertation, written by Vishal Musaramthota, and entitled as Prediction of Fracture Toughness and Durability of Adhesively Bonded Composite Joints with Undesirable Bonding Conditions, having been approved in respect to style and intellectual content, is referred to you for judgment.

We have read this dissertation and recommend that it be approved.

Dwayne McDaniel

Jose R. Almirall

Xiangyang Zhou

Benjamin Boesl, Co-Major Professor

Norman D.H. Munroe, Co-Major Professor

Date of Defense: November 02, 2015

The dissertation of Vishal Musaramthota is approved.

Interim Dean Ranu Jung

College of Engineering and Computing

Andrés G. Gil

Vice President for Research and Economic Development and Dean of the University Graduate School

Florida International University, 2016 
(C) Copyright 2016 by Vishal Musaramthota

All rights reserved. 


\section{DEDICATION}

This dissertation is dedicated to my ever loving parents for their invaluable sacrifices and encouragements. Their words of support and encouragement during the challenges of graduate school and life will always ring in my ears. I am truly thankful for having them in my life. This work is also dedicated to my sisters, for their everlasting love and providing me with the moral support I needed without which I would have not been able to accomplish my doctoral dissertation successfully. My appreciation extends to my all my friends for their love and support.

A special feeling of gratitude and gratefulness to my dear friend mentioned below who supported me through thick and thin of my $\mathrm{PhD}$ career.

Keerthi Pattem 


\section{ACKNOWLEDGMENTS}

I take this opportunity to express my sincere gratitude towards Dr. Dwayne McDaniel (Committee Member and PI of this research project) for his support, motivation and valuable discussions during this entire research work. His continuous guidance helped me immensely in the research. I thank him for long discussion hours he dedicated just to clear the knots in this research. His ideas always gave a new dimension to the research, and proposed many different aisles of solution. He taught me of doing things efficiently and to achieve maximum output. He has taught me to challenge myself and stretch my limitations. I am thankful for his efforts, time and dedication for completion of my dissertation and developing my career.

My sincere thanks go to Dr. Norman Munroe (Major Professor) for his continuous guidance in developing me as a researcher. A simple thank you is not enough to express my gratitude towards him. His untiring support and encouragement throughout my Ph.D. has made the path very clear and easy. My deepest gratitude goes to him for providing opportunity, direction and motivation in the various research domains that he allowed me to work for. His ever-enthusiastic spirit, critical perspicacity, and energetic approach were the sole motivation of my persistence.

My gratitude also extends to Dr. Benjamin Boesl (Co-Major Professor) for the genuine interest he expressed in my research work along with serving as a co-major professor in my dissertation committee. His experience shared during my proposal defense has helped me to undertake more research strategies. He has always been very supportive and encouraging and provided a big help with his research facilities. Much learning was gained through attending his insightful discussions and his valuable suggestions.

Special thank you to Dr. Xiangyang Zhou (Committee member) for letting me utilize his research facilities at University of Miami throughout this research. He has always been very supportive and was always available to guide me through my research. I thank Dr. Jose Almirall (Committee member) for his support throughout this research and providing me with his valuable 
insights on characterization techniques that can be adopted to solve issues. This dissertation benefited greatly from his input. He always encouraged me on employing critical thinking in my analyses.

I would like to acknowledge Applied Research Center (ARC), Mechanical and Materials Engineering Department (MME), Advanced Materials Engineering Research Institute (AMERI), Florida Center for Analytical Electron Microscopy (FCAEM) and University of Miami (UM) for laboratory support. I thank the University Graduate School for extending a Doctoral Evidence Acquisition (DEA) and Dissertation Year Fellowship (DYF) fellowships to support my research. I also acknowledge the financial support from Federal Aviation Administration Cooperative Agreement 12-C-AM-FIU.

A special thank you is extended to a good friend and colleague Tomas Pribanic, research analyst (Applied Research Center), for generously imparting his technical knowledge and brilliant ideas and for his moral support throughout this research. By mimicking his personality, of constructive criticism and a tireless work ethic, I was able to conduct my research in a more efficient and systematic manner. Additionally, I would like to extend my thanks to Anthony Abrarho and Dr. Aparna Aravelli for their help and support during this research period.

I need to make a separate paragraph for my friends here. Puneet, Sushma and Chandan who have been very helpful in my early years of Ph.D. and continuing till date. Special adoration to Keerthi and admiration to Aishwharya, Sudheer, Raghavendra and Bala who are always willing to talk to me endlessly. The gratitude extends further to my dear friend Kavitha, who you cannot stop loving. She supported me through the time I've known her and an epicenter for all my food cravings. Thanks to Neha and Mouna for always being there for me and frustrating me throughout. All you guys have been amazing and very special, which I treasure always. I cannot imagine my university years or indeed the years to follow without your friendship. 
ABSTRACT OF THE DISSERTATION

PREDICTION OF FRACTURE TOUGHNESS AND DURABILITY OF ADHESIVELY

BONDED COMPOSITE JOINTS WITH UNDESIRABLE BONDING CONDITIONS

by

\author{
Vishal Musaramthota \\ Florida International University, 2016 \\ Miami, Florida \\ Professor Norman Munroe, Co-Major Professor \\ Professor Benjamin Boesl, Co-Major Professor
}

Advanced composite materials have enabled the conventional aircraft structures to reduce weight, improve fuel efficiency and offer superior mechanical properties. In the past, materials such as aluminum, steel or titanium have been used to manufacture aircraft structures for support of heavy loads. Within the last decade or so, demand for advanced composite materials have been emerging that offer significant advantages over the traditional metallic materials. Of particular interest in the recent years, there has been an upsurge in scientific significance in the usage of adhesively bonded composite joints (ABCJ's). ABCJ's negate the introduction of stress risers that are associated with riveting or other classical techniques. In today’s aircraft transportation market, there is a push to increase structural efficiency by promoting adhesive bonding to primary joining of aircraft structures. This research is focused on the issues associated with the durability and related failures in bonded composite joints that continue to be a critical hindrance to the universal acceptance of ABCJ's. Of particular interest are the short term strength, contamination and long term durability of ABCJ's.

One of the factors that influence bond performance is contamination and in this study the influence of contamination on composite-adhesive bond quality was investigated through the 
development of a repeatable and scalable surface contamination procedure. Results showed an increase in the contaminant coverage area decreases the overall bond strength significantly. A direct correlation between the contaminant coverage area and the fracture toughness of the bonded joint was established. Another factor that influences bond performance during an aircraft's service life is its long term strength upon exposure to harsh environmental conditions or when subjected to severe mechanical loading. A test procedure was successfully developed in order to evaluate durability of ABCJ's comprising severe environmental conditioning, fatiguing in ambient air and a combination of both. The bonds produced were durable enough to sustain the tests cases mentioned above when conditioned for 8 weeks and did not experience any loss in strength. Specimens that were aged for 80 weeks showed a degradation of $10 \%$ in their fracture toughness when compared to their baseline datasets. The effect of various exposure times needs to be further evaluated to establish the relationship of durability that is associated with the fracture toughness of ABCJ's. 


\section{TABLE OF CONTENTS}

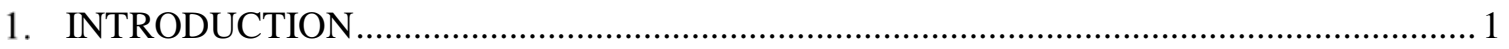

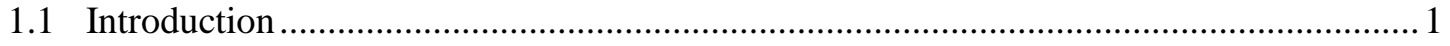

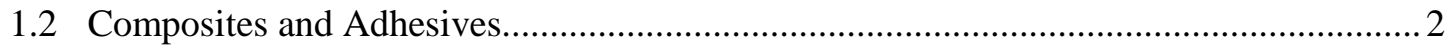

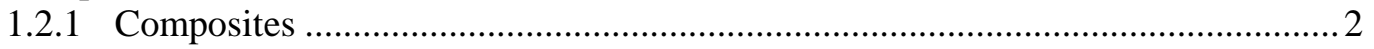

1.2.1.1 Classification of Composites .................................................................................

1.2.1.2 Economic aspects of Composites and their commercial products: .....................6

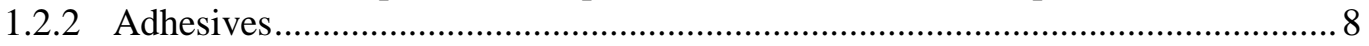

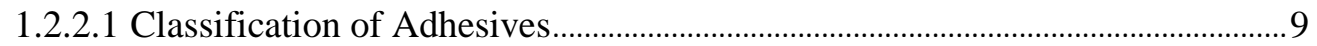

1.2.2.2 Economic aspects of Adhesives and Commercial Products .............................. 10

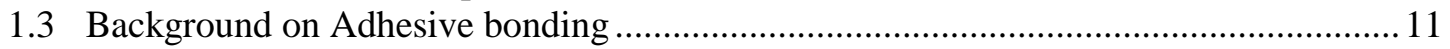

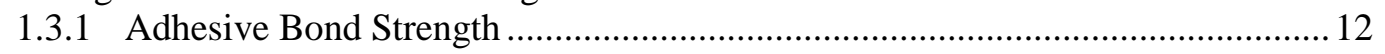

1.3.2 Mechanisms of Adhesive Bonding ................................................................. 15

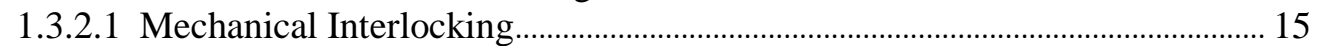

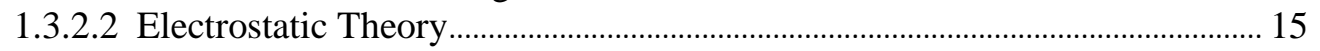

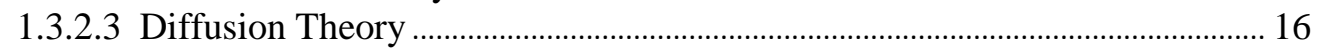

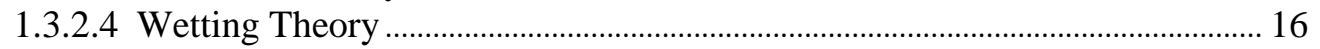

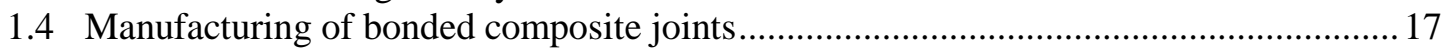

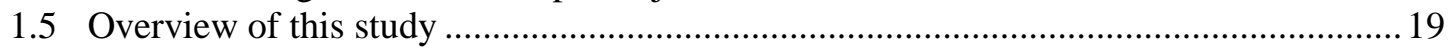

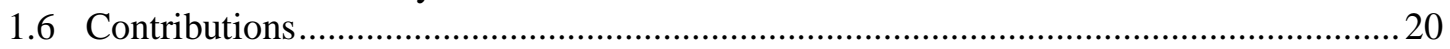

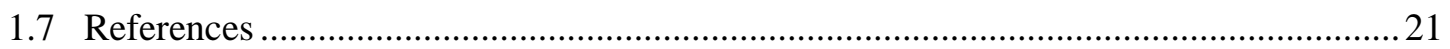

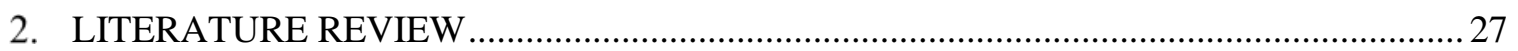

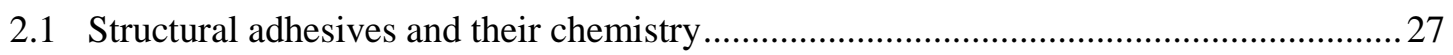

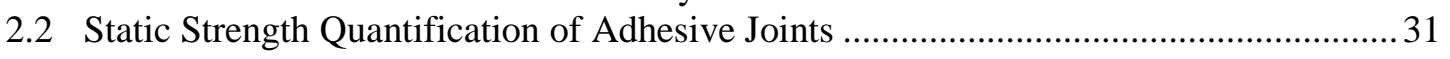

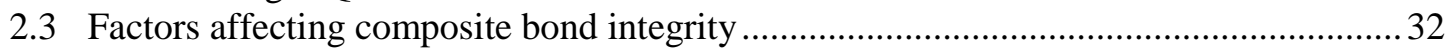

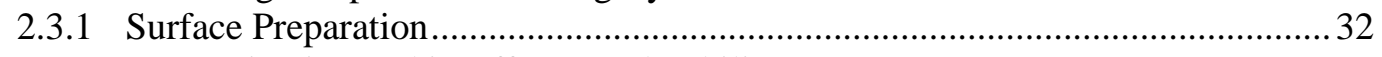

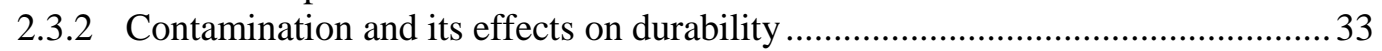

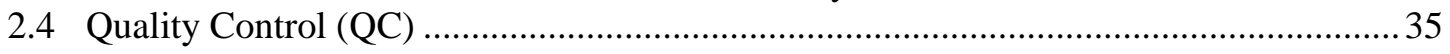

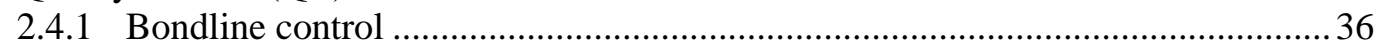

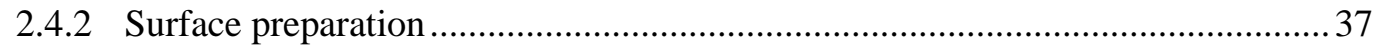

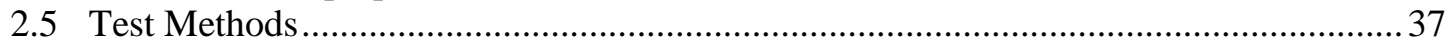

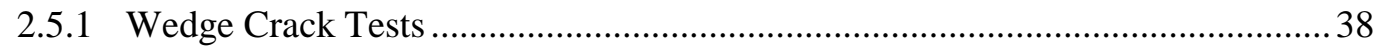

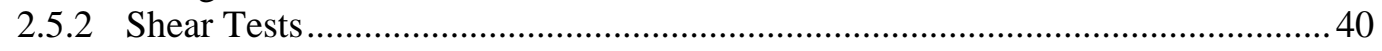

2.5.2.1 Interlaminar shear strength (ILSS) Tests ............................................................ 41

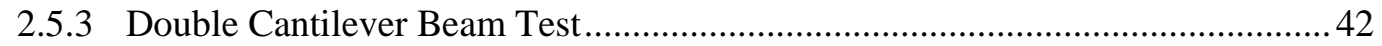

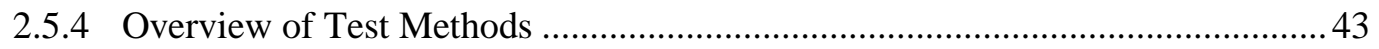

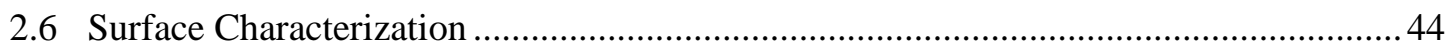

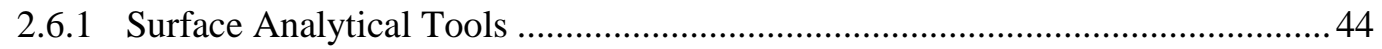

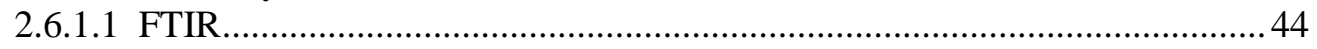

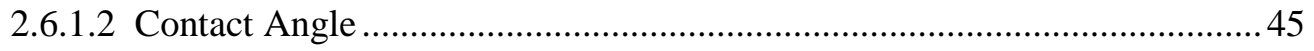

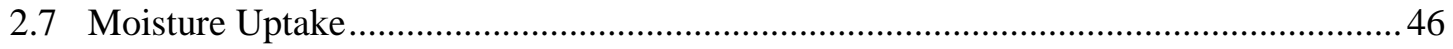

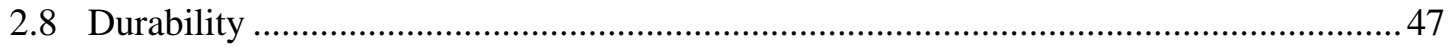

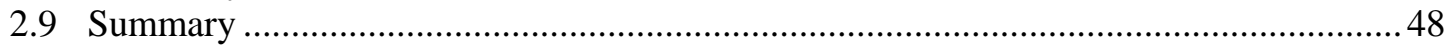

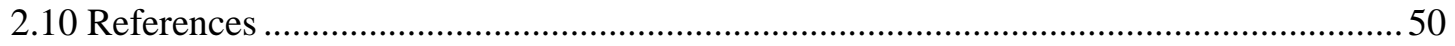




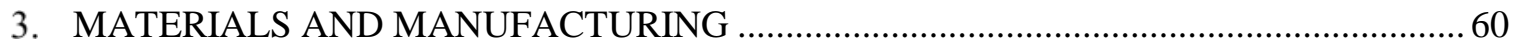

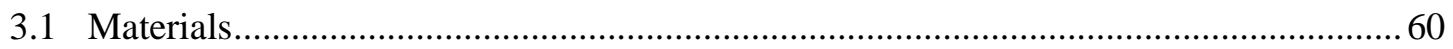

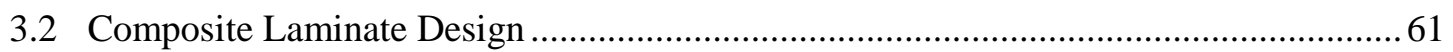

3.3 Manufacturing of Adhesively Bonded Composite Laminates ............................................61

3.4 Preparation of Contaminated Adhesively Bonded Composite Laminates .........................68

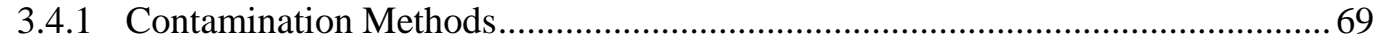

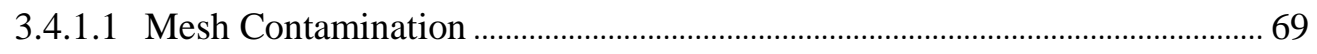

3.4.1.2 Stamp Contamination …………………………………………………. 70

3.4.2 Contaminant Materials................................................................................... 71

3.4.2.1 Aerosol Contaminant ................................................................................... 71

3.4.2.2 Frekote Contaminant ................................................................................ 72

3.4.3 Contamination Symmetry ………………………………………………..... 72

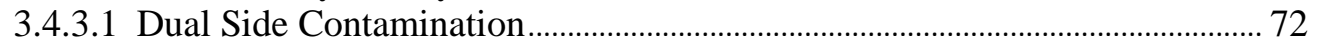

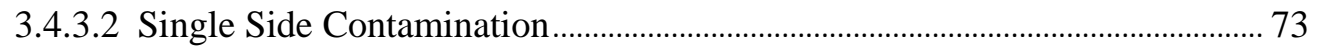

3.4.4 Contamination Levels .................................................................................. 73

3.4.4.1 Stamp with Small Area (A1) .............................................................................. 73

3.4.4.2 Stamp with Larger Area (A3) .................................................................... 73

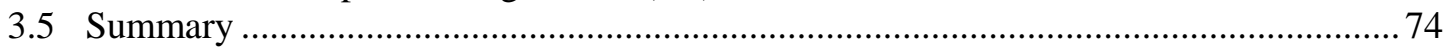

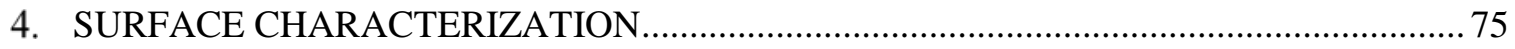

4.1 Experimental Approach......................................................................................

4.1.1 Wettability Measurements .............................................................................. 76

4.1.2 FTIR Measurements ................................................................................

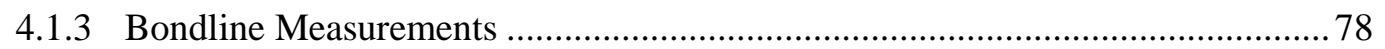

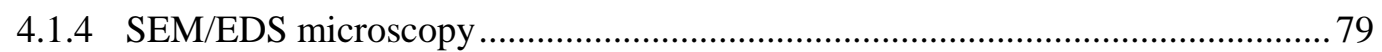

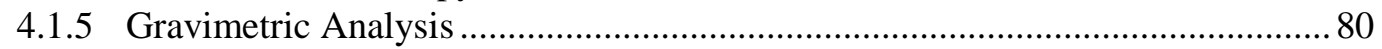

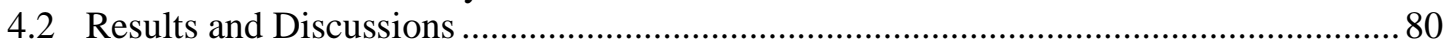

4.2.1 Wettability Measurement.................................................................................. 80

4.2.1.1 Surface Energy measurements.................................................................................. 83

4.2.2 FTIR Measurements ...................................................................................... 85

4.2.2.1 FTIR Spectroscopy on adherend................................................................. 85

4.2.2.2 FTIR spectroscopy on adhesive........................................................................ 88

4.2.2.3 FTIR spectroscopy on contaminated specimens........................................... 90

4.2.3 Gravimetric analysis .................................................................................. 92

4.2.4 Bondline Measurements ............................................................................... 94

4.2.5 SEM/EDS microscopy ……………………………............................... 95

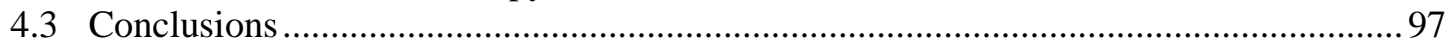

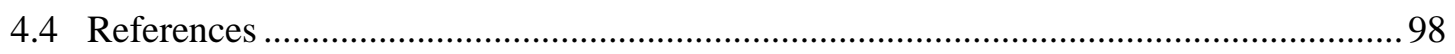

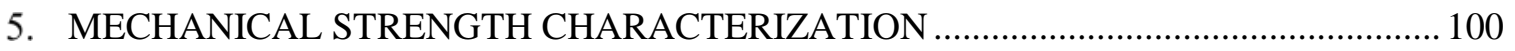

5.1 Fracture Toughness Evaluation.................................................................................. 100

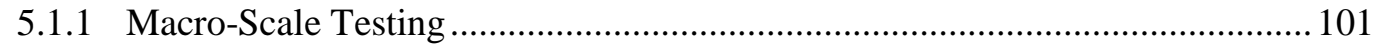

5.1.2 Micro-Scale In-Situ Testing................................................................................ 103

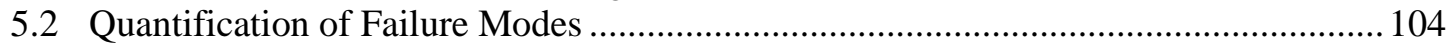

5.2.1 Modeling Procedure/Digital Image Processing ................................................... 105

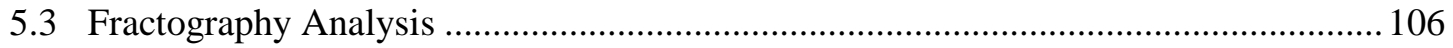

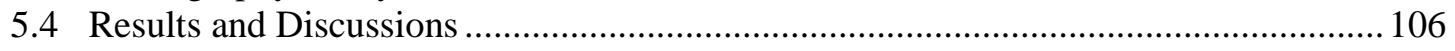

5.4.1 Micro-Scale Testing (ENF Testing/In-situ electron microscopy)....................... 107 
5.4.2 Macro-Scale Testing (DCB Testing) …....................................................... 108

5.4.2.1 Fracture Toughness Evaluations for Various Contamination Levels ..........110

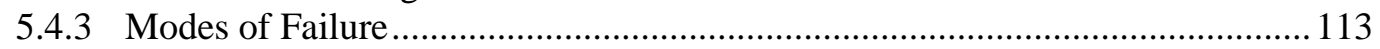

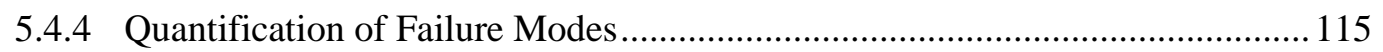

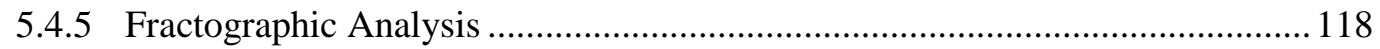

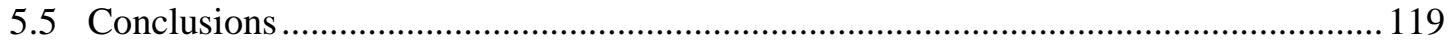

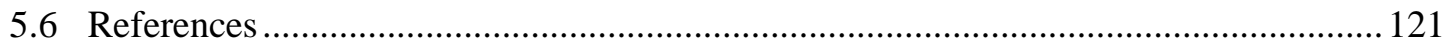

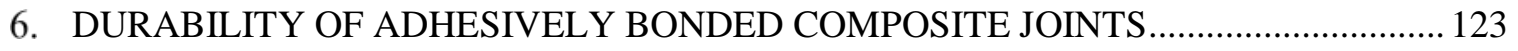

6.1 Development of Durability Test Procedure.................................................................... 123

6.1.1. Moisture Absorption Studies of Composite Laminates and ABCJ's...................125

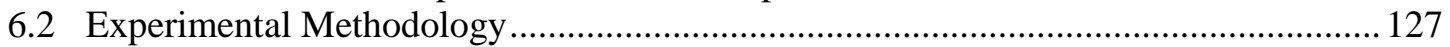

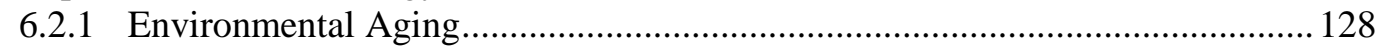

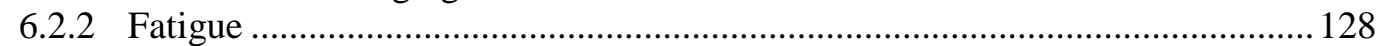

6.2.3 Combined Environmental Aging and Fatigue .................................................. 130

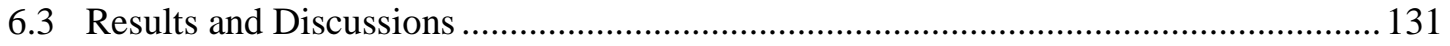

6.3.1 Mechanical Strength Characterization for Durability Specimens......................131

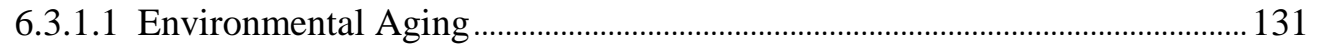

6.3.1.2 Fatigue in Ambient Air........................................................................................132

6.3.1.3 Combined Environmental Aging and Fatigue..................................................133

6.3.2 Assessing the Mode of Failure for Conditioned Specimens ............................... 135

6.3.2.1 Non-Contaminated specimens subjected to Durability..............................135

6.3.2.2 Contaminated specimens subjected to Durability ........................................137

6.3.2.2.1. A1 contaminated specimens subjected to durability............... 138

6.3.2.2.2. A3 contaminated specimens subjected to durability ..............140

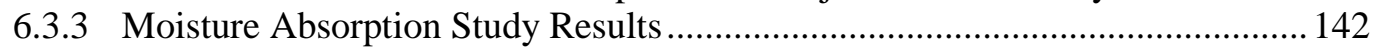

6.3.3.1. Fracture Toughness evaluations for Long Exposure specimens ................ 143

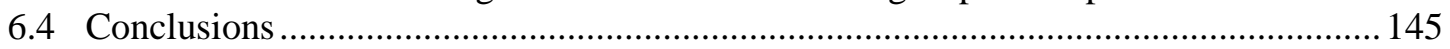

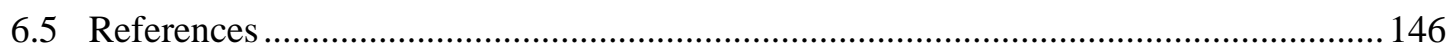

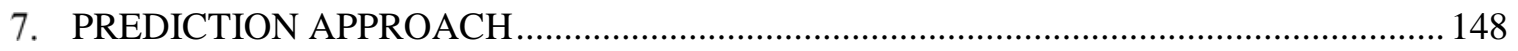

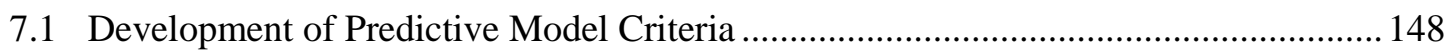

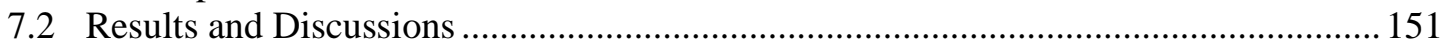

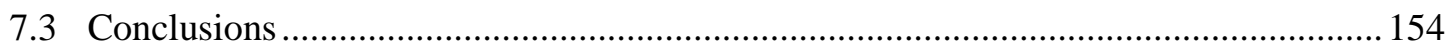

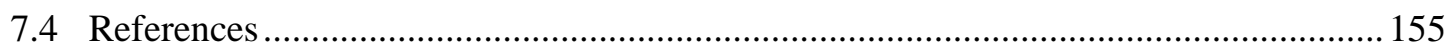

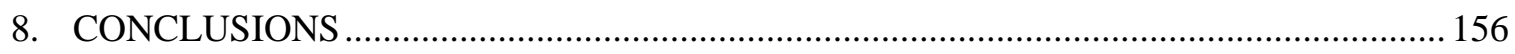

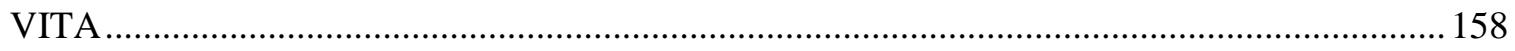




\section{LIST OF TABLES}

TABLE

PAGE

Table 3.1: List of Materials utilized during composite laminate manufacturing ........................... 65

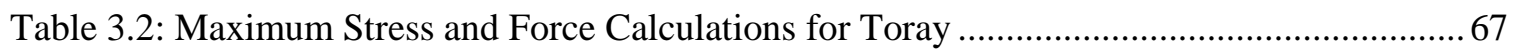

Table 3.3: Displacement and Force Dependency on Number of Plies ......................................... 68

Table 4.1: Wetting Angles with DI Water, Ethylene Glycol and Diiodomethane for Aerosol mesh and stamp contaminated specimens

Table 4.2: Wetting Angles with DI Water, Ethylene Glycol and Diiodomethane for Pristine and Frekote stamp contaminated specimens. 84

Table 4.3: Surface Free Energy components of pristine and contaminated specimens .85

Table 4.4: Assignment of the Infrared absorption of epoxy resin with and without contaminant..

Table 4.5: Percent weight gain of laminate comparison between mesh and stamp approach ....... 92

Table 4.6: Percent weight gain of contaminant on laminate between Single side and Dual side contamination.

Table 4.7: Average bondline thickness (mm) for all the test cases mentioned in section 3.4 ....... 94

Table 5.1: Standards and Protocols for Mode I, ModeII and mixed-mode fracture. 100

Table 5.2: Bondline thickness, critical energy release rate $\left(\mathrm{G}_{1 \mathrm{C}}\right)$, and cohesive failure ratio of macroscale specimen (DCB) from baseline and contaminated samples 


\section{LIST OF FIGURES}

FIGURE

PAGE

Figure 1.1: Specific Stiffness vs Specific Strength of CFRP composites [31] .............................

Figure 1.2: Comparison of conventional materials to that of composite materials [35]................ 4

Figure 1.3: Schematic of a Unidirectional Fiber Composite [37] ............................................. 5

Figure 1.4: Market growth of CFRP and its predictions in various domains [40] ........................ 6

Figure 1.5: Compound Annual Growth Rate (CAGR) of CFRP market and its predictions [40]...7

Figure 1.6: CFRP constituting majority of Airbus A380 aircraft structure [44]............................ 8

Figure 1.7: Global Structural adhesives market share by product, 2012-2022 [52] ..................... 11

Figure 1.8: Bonding fabrication process of aerostructures [61] ................................................ 12

Figure 1.9: A typical bonded joint showing adherends bonded with adhesive............................ 13

Figure 1.10: Typical bond failure occurrences dictating the type of failure a) adhesive failure;

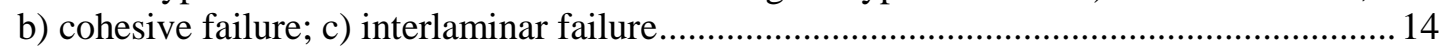

Figure 1.11: Crack Opening Modes ................................................................................... 14

Figure 1.12: Schematic showing the interlocking effect of the adhesive into the adherend.......... 15

Figure 1.13: Schematic showing the charge transfer mechanism between adhesive and

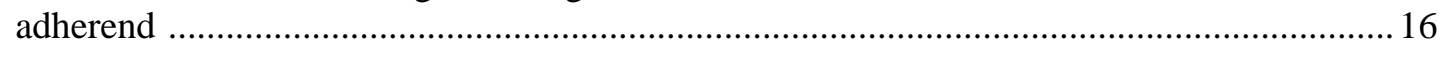

Figure 1.14: Schematic showing the interdiffusion of adhesive and adherend.............................16

Figure 1.15: Schematic showing polymeric chains interacting with the substrate i.e. Vander

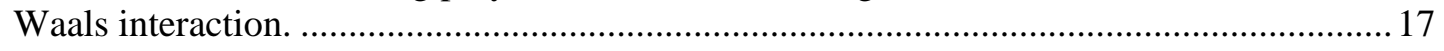

Figure 2.1: Synthesis of di-glycidyl ether of bis-phenol-A with epichlorohydrin........................ 28

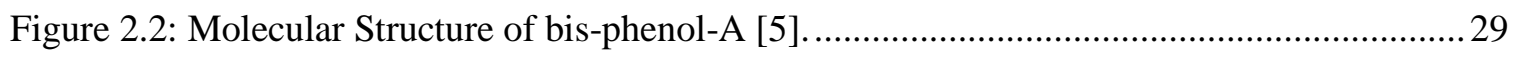

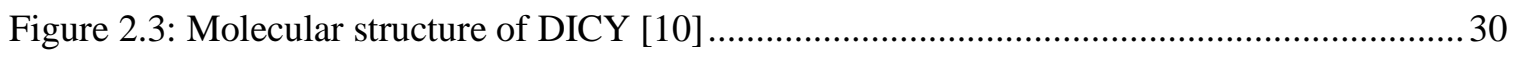

Figure 2.4: Schematic showing Wedge Crack Extension Test [65]........................................... 39

Figure 2.5: Schematic Showing Lap Shear test coupon......................................................... 41

Figure 2.6: Schematic of a Double Cantilever Beam test specimen ............................................ 43 
Figure 3.2: a) Dry prepreg laminate sheets cut to the desired dimensions and b) Stack of 10 plies of prepreg laminate.

Figure 3.3: a) Application of Peel ply fabric on to the stacked laminate; b) breather material on top of peel ply covered stacked laminate.

Figure 3.4: Vacuum applied to the vacuum bagging process ...................................................... 64

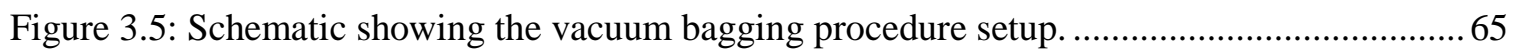

Figure 3.6: Image of an autoclave utilized for composie manufacturing..................................... 66

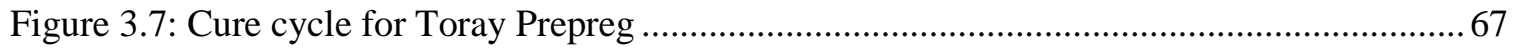

Figure 3.8: Adhesive cure cycle of bonded composite laminates ............................................... 68

Figure 3.9: Contamination Procedure- Mesh Approach ............................................................ 70

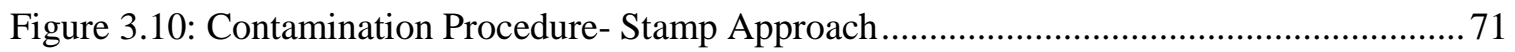

Figure 3.11: Differences in the type of approaches a) Mesh; and b) Stamp ................................. 72

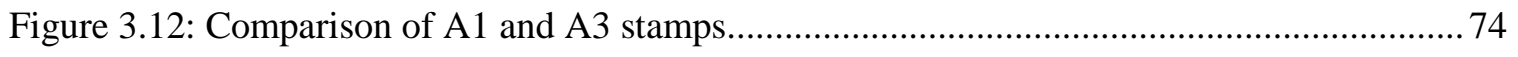

Figure 4.1: Surface characterization techniques conducted in the current research. ..................... 75

Figure 4.2: (a) Kyowa Contact angle meter and (b) Wetting angle made by a liquid over a

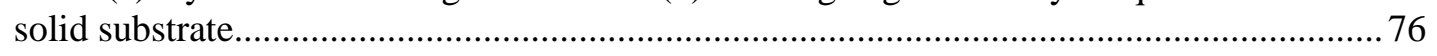

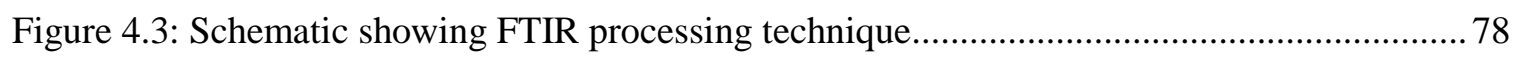

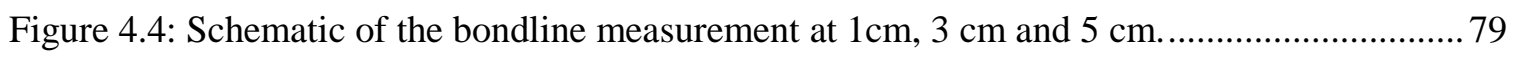

Figure 4.5: Contact Angle measurements for mesh and stamp approach using aerosol contaminant.

Figure 4.6: Contact angle measurements for baseline, aerosol (mesh and stamp) and Frekote

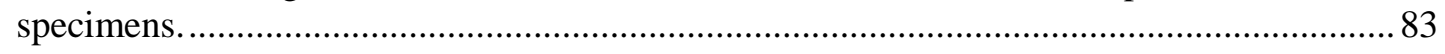

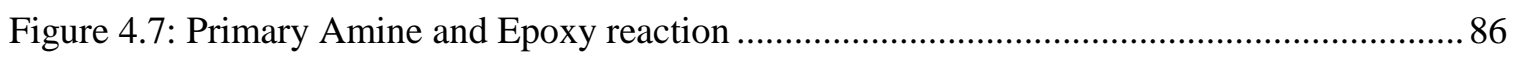

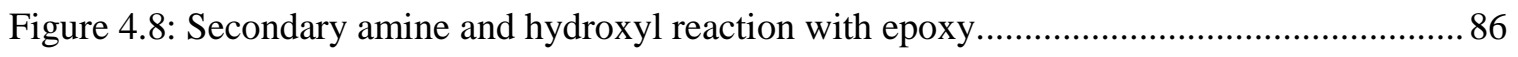

Figure 4.9: Comparison of FTIR Spectra of Toray adherend before and after cure

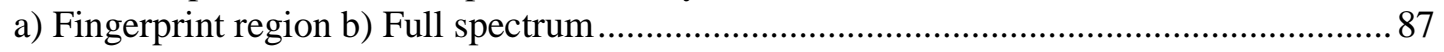

Figure 4.10: Tertiary Amine- Epoxy reactions followed by etherification..................................... 88 
Figure 4.11: Comparison of FTIR Spectra of AF555M adhesive before and after cure a)

Fingerprint region b) Full spectrum.

Figure 4.12: FTIR spectra comparing the Frekote contaminant, pristine composite surface and

Frekote contaminated composite surface

Figure 4.13: Expanded view of influencial bands in contaminated specimens in orange.

Figure 4.14: Weight gain comparisons between Aerosol and Frekote contaminant.

Figure 4.15: Bondline measurements conducted on a pristine specimen.

Figure 4.16: SEM image showing the interface of a non-contaminated specimen. 95

Figure 4.17: SEM image showing the interface of a contaminated specimen. 96

Figure 4.18: a) Elemental line scans of $\mathrm{Si}$ in the baseline/non-contaminated region and b)

Elemental line scans of $\mathrm{Si}$ in the contaminated region across the interface. 96

Figure 5.1: DCB Experimental setup with bonded specimen under load....

Figure 5.2: Complinace correction using Modified Beam Theory (MBT).

Figure 5.3 (a) Schematic of in-situ end notch fracture (ENF) test configuration (b) Low magnification SEM image of an ENF sample loaded in the 4 point bend fixture. (c) Higher magnification image of the crack tip region (false green coloring was added to delineate the region of adhesive.

Figure 5.4. Load Displacement curves for baseline (non-contaminated) and A3 contaminated specimen using in situ ENF testing.

Figure 5.5: Analysis of the damage initiation mechanisms for baseline and A3 contaminated specimens prior to loading and at peak load.

Figure 5.6: $\mathrm{G}_{\mathrm{IC}}$ values between baseline (non-contaminated), Aerosol (mesh and stamp) and Freekote (stamp).

Figure 5.7: Comparison of $\mathrm{G}_{\mathrm{IC}}$ values between baseline (non-contaminated), dual side contamination and single side contamination with Frekote.

Figure 5.8: GIC vs Cohesive Failure Ratio of Baseline, A1 Stamp and A3 Stamp.

Figure 5.9: GIC vs Cohesive Failure Ratio of Baseline, A1 Low Pressure and A1

High Pressure.

Figure 5.10: Failure Modes a) Baseline (non-contaminated); b) A1 Low Pressure;

c) A3 Stamp and d) A1High Pressure

Figure 5.11: Quantified images determining cohesive and adhesive ratios. Top row- fractured surfaces for each of the four configurations. Bottom row- Illustrated computed areas 
images using ImageJ, red coloration indicates cohesive failure, black indicates

adhesive/interlaminar failure, and green demarcates the boundaries between each.

Figure 5.12: Example cases of the effect of (a) contamination area and (b) contamination size on cohesive failure ratio and critical energy release rate values. Contaminated area plays a major role in decreasing the bond quality whereas the effects of contamination size are negligible.

Figure 5.13: Line profile analysis showing a line drawn over the fractured surface a) baseline specimen; b) A1L stamp specimen and c) A3 stamp specimen. Line profile path is shown beneath the optical image follwed by their spectral mapping.

Figure 6.1: Durability Assessment Procedure

Figure 6.2: Bonded and Non-bonded specimen placed in an environmental chamber for moisture uptake analysis.

Figure 6.3: a) Environmetal chamber on left and b) Inside an environmental chamber 128

Figure 6.4: Schematic set-up of the fatigue fixture. 129

Figure 6.5: Fatigue fixture for DCB specimens- specimen loaded with 0.5 inch a) forward amplitude; and b) backward amplitude.

Figure 6.6: Fatigue fixture inside an environmental chamber.

Figure 6.7: Non-Contaminated and Contaminated specimen subjected to Environmental Exposure

Figure 6.8: Non-Contaminated and Contaminated specimen subjected to mechanical fatigue...133

Figure 6.9: Non-Contaminated and Contaminated specimen subjected to combined loading (environmental exposure and mechanical fatigue simultaneously).

Figure 6.10: Comparison of mode of failures for non-contaminated specimens that underwent durability route.

Figure 6.11: Effect of Durability on CTOD for non-contaminated specimens......

Figure 6.12: Comparison of mode of failures for A1 contaminated specimens that are subjected to durability.

Figure 6.13: Effect of Durability on CTOD for A1contaminated specimens

Figure 6.14: Comparison of mode of failures for A3 contaminated specimens that are subjected to durability.

Figure 6.15: Effect of Durability on CTOD for A1contaminated specimens 142 
Figure 6.16: Moisture uptake \% plot for bonded composite laminate and laminate only

Figure 6.17: Effect of moisture ingression on bond strength of bonded and non-bonded specimens with varying exposure times.

Figure 6.18: Mode of Failure for long exposure specimens bonded with adhesive. 145

Figure 7.1: Schematic of the true representation of the contamination link at the 149

Figure 7.2: RVE Unit cell drawn in red with a local coordinate. 150

Figure 7.3: Dependency of unit cell cohesion area on Kc 152

Figure 7.4: GC dependency on RVE Unit cell cohesive area... 152

Figure 7.5: $\mathrm{G}_{1 \mathrm{C}}$ Experimental vs Predicted Slopes..... 153

Figure 7.6: Predicted vs Experimental Fracture Toughness plots. 154 


\section{ABBREVIATIONS}

ASTM

ABCJ

ATR- FTIR

BOPACS

CAGR

CAI

CA

CBT

CCM

CFRP

$\mathrm{CM}$

CTOD

DCB

DGEBA

DICY

DOD

EASA

ECM

EDS

ENF

EU

FAA
American Society for Testing Materials

Adhesively Bonded Composite Joint

Attenuated Total Reflectance Fourier Transform Infrared Spectroscopy

Boltless assembling Of Primary Aerospace Composite Structures

Compound Annual Growth Rate

Composites Affordability Initiative

Contact Angle

Corrected Beam Theory

Compliance Calibration Method

Carbon Fiber Reinforced Polymer

Compression Molding

Crack Tip Opening Displacement

Double Cantilever Beam

Diglycidyl ether of bisphenol-A

Dicyandiamide

Department of Defense

European Aviation Safety Agency

Experimental Compliance Method

Energy Dispersive Spectroscopy

End Notch Flexural

European Union

Federal Aviation Administration 


\begin{tabular}{|c|c|}
\hline FAW & Fiber Areal Weight \\
\hline FB & Flash Breaker \\
\hline FP & Fractional Polarity \\
\hline FTIR & Fourier transform infrared spectroscopy \\
\hline HMW & High Molecular Weight \\
\hline ILSS & Interlaminar Shear Strength \\
\hline IPSS & In-Plane Shear Strength \\
\hline LCM & Liquid Composite Molding \\
\hline LS & Lap Shear \\
\hline MCC & Modified Compliance Calibration \\
\hline MSDS & Material Safety Data Sheet \\
\hline MBT & Modified Beam Theory \\
\hline MP & Mechanical Polishing \\
\hline NDE & Non-Destructive Evaluation \\
\hline NDI & Non-Destructive Inspection \\
\hline NDT & Non-Destructitve testing \\
\hline PAN & Poly Acrylic Nitrile \\
\hline PABST & Primary Adhesively Bonded Structure Technology \\
\hline PDMS & Polydimethylsiloxane \\
\hline PTFE & Polytetrafluoroethylene \\
\hline PMC & Polymer Matrix Composites \\
\hline PSA & Pressure Sensitive Adhesives \\
\hline PVA & Poly-Vinyl Alcohol \\
\hline QC & Quality Control \\
\hline
\end{tabular}




\begin{tabular}{ll} 
ROI & Region of Interest \\
RC & Resin Content \\
RH & Relative Humidity \\
RTM & Resin Transfer Molding \\
RVE & Representative Volume Element \\
SBT & Simple Beam Theory \\
SEM & Scanning Electron Microscopy \\
SFE & Surface Free Energy \\
SRIM & Structural Reaction Injection Molding \\
TCA & Toray Composites America \\
TDCB & Tapered Double Cantilever Beam \\
UBC & Undesirable Bonding Conditions \\
UTM & Ultimate Tensile Machine \\
UV & Ultraviolet \\
VARTM & Vacuum Assisted Resin Transfer Molding \\
VIP & Vacuum Infusion Processing \\
\hline
\end{tabular}




\section{LIST OF SYMBOLS}

\begin{tabular}{|c|c|}
\hline A & Surface Area \\
\hline$\omega$ & Deflection \\
\hline $\mathrm{F}$ & Load \\
\hline 1 & Length \\
\hline $\mathrm{E}$ & Young's Modulus \\
\hline I & Inertia \\
\hline$\gamma_{\mathrm{lv}}$ & Surface Energy of liquid-vapor interface \\
\hline$\gamma_{\mathrm{sv}}$ & Surface Energy of solid-vapor interface \\
\hline$\gamma_{\mathrm{sl}}$ & Surface Energy of solid-liquid interface \\
\hline$\Theta$ & Contact angle \\
\hline $\mathrm{mJ}$ & Milli-Joule \\
\hline $\mathrm{m}$ & Meter \\
\hline $\mathrm{cm}$ & centimeter \\
\hline $\mathrm{G}_{\mathrm{I}}$ & Strain Energy Release Rate \\
\hline $\mathrm{P}$ & Load \\
\hline$\delta$ & Displacement \\
\hline $\mathrm{b}$ & Width of a DCB specimen \\
\hline $\mathrm{a}$ & Crack length \\
\hline $\mathrm{C}$ & Compliance \\
\hline$\Delta$ & Correction factor for compliance \\
\hline $\mathrm{M}_{\mathrm{i}}$ & Final weight of specimen after exposure \\
\hline $\mathrm{M}_{\mathrm{o}}$ & Initial weight of specimen after exposure \\
\hline
\end{tabular}




$\begin{array}{cl}\mathrm{Y}_{\mathrm{g}} & \text { Length scale in global coordinate system } \\ y_{l} & \text { Length scale in local coordinate system } \\ \epsilon & \text { Scaling parameter } \\ \mathrm{a}_{\mathrm{f}} & \text { Radius of the circular flaw } \\ \mathrm{d} & \text { Height of the flaw } \\ \rho & \text { Tip radius of the circular flaw } \\ \mathrm{S} & \text { Applied stress } \\ \sigma_{\mathrm{y}} & \text { Stress field } \\ \mathrm{K}_{\mathrm{c}} & \text { Stress intensity factor } \\ \sigma_{\mathrm{s}} & \text { Applied stress } \\ A_{\text {Unitcell }} & \text { Area of the Unit Cell } \\ A_{\text {cohesive }} & \text { Area of the cohesive region in a unit cell } \\ A_{\text {adhesive }} & \text { Area of the adhesive region in a unit cell }\end{array}$




\section{INTRODUCTION}

\subsection{Introduction}

Flying is considered to be the primary mode of transportation with millions of people travelling every year. There has been a 50\% increase in the number of passengers in the last ten years and is continuously increasing exponentially [1]. Hence, airlines need an expansion in their flight volume with bigger capacities such as the Airbus A380. The A380 is currently the largest aircraft in the world with a carrying capacity of greater than 800 passengers and a take-off weight of 560 tons [2] which is a significant improvement when compared to conventional aircrafts [3]. With a structure of such giant magnitude, enormous forces act on the wings as well as the aircraft by itself. Specialized steels and aluminum alloys can withstand such large forces at the expense of additional weight [4]. With diverse research and development over the past decades, the aircraft community has gained significant knowledge and understanding of the uses, strengths and limitations of these materials.

In the past, materials such as aluminum, steel or titanium have been used to manufacture complex engineering structures for support of heavy loads [4, 5]. Within the last decade or so, demand for new materials $[6,7]$ that are less dense and exhibit exceptional mechanical properties have been developed which offer significant advantages over the traditional metallic materials [8, 9]. These new materials include carbon fiber reinforced polymer (CFRP) composites. Composite materials offer advantages over conventional materials used in aerospace structures due to their high strength-to-weight ratio $[10,11,12]$. They provide material toughness, improved damage tolerance, fatigue endurance and have lower weights (50\% less than that of steel) [11]. Despite the superior material properties, there still exist challenges associated with joining of CFRP components. In the recent years, there has been an upsurge in scientific interest of using adhesively bonded composite joints in a host of fields including aircraft, automobiles etc. The optimal joining of composites is accomplished using adhesive bonding, which negates the introduction of stress 
risers that are associated with riveting or other classical techniques [11, 13]. In today's aircraft transportation market, there is a push to increase structural efficiency by eliminating secondary joining via adhesive bonding [11] and to use the latter as the primary joining technique. Adhesively bonded composite joints (ABCJs) are expected to sustain static and cyclic loads for extended periods of time without having negative effects on the load bearing capacity of the structure [14].

Recently, the use of adhesive bonding of composite structures has generated a great deal of interest in the bonding community. Of particular interest is the short term strength and long term durability of these adhesively bonded systems. Issues associated with the durability and related failures in aircrafts, continue to be a critical hindrance to the universal acceptance of ABCJs [15, 16]. Numerous studies have been conducted to determine the initial bond strength of ABCJs, yet there is still limited understanding on the assessment of their long term strength. The extrinsic factors that affect the strength and durability of ABCJs are environmental conditions [17], operating temperatures [18, 19], relative humidity [20], service loads [21] and cleanliness of bonding surface $[22,23]$.

Contamination and its sources are also a global concern in the production of durable adhesive bonds. Sources of possible contamination include peel ply residues, release agents, skin oil, dust and moisture [24]. The contamination from peel ply and their release agents have been shown to significantly degrade bond quality, which results in reduced adhesion of composite systems [25, 26, 27, 28]. Thus, there is a great need for the assessment of the effects of contamination on bond strength and its durability in ABCJs. This is the primary focus of this research.

\subsection{Composites and Adhesives}

\subsubsection{Composites}

Over the past few decades, lighter materials with improved toughness have been developed [29] where the goal was primarily to achieve weight savings for an airframe structure. CFRP 
materials show greater promise in the aforementioned properties via increased specific strength or stiffness [8], as described in Figure 1.1. The reduction in aircraft weight by itself results in fuel efficiency and lower carbon dioxide emissions along with increased range and maneuverability and higher pay load. These are usually continuous carbon fibers (filamentary phase) embedded in a polymeric epoxy (matrix phase) [30].

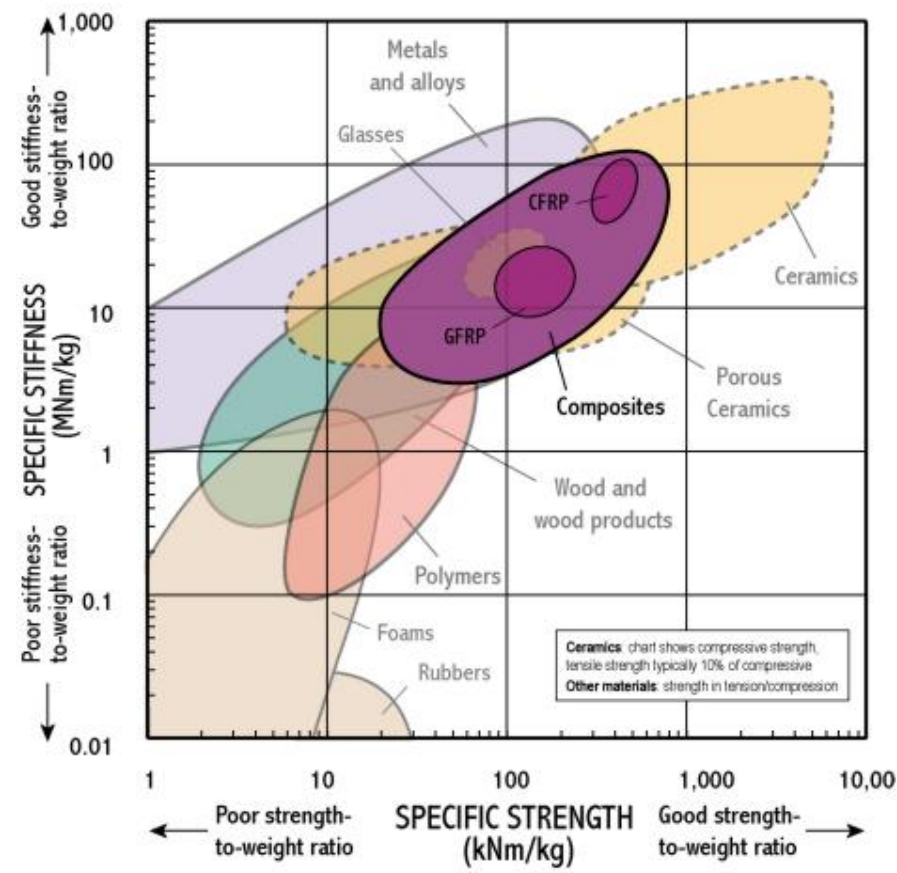

Figure 1.1: Specific Stiffness vs Specific Strength of CFRP composites [31].

Carbon fiber reinforced materials have the advantage of offering highly complex shapes, more complex than possibly using steel or aluminum [32]. CFRP materials can outperform metals in terms of their material performances, offering diverse properties that no one material is able to provide. For example, these materials are light in weight yet an improved strength and stiffness was observed when compared to metal alloys or polymers as depicted in Figure 1.1. Additionally, a CFRP material, when stacked in a preferential order, offers high structural stability in conjunction with possessing higher tensile strength and modulus in the direction of the fiber [33]. Fiber reinforced composites in aerospace applications offer improved performance i.e. smoother and 
more aerodynamic form can be achieved with special aeroelastic properties; improved damage tolerance and reduced detectability. Additionally fiber composites offer superior durability by providing resistance to fatigue, corrosion and mechanical damage [34]. Figure 1.2 below, showcases a few of the properties such as weight, thermal expansion, stiffness and strength aspects of composite materials, pictorially. As mentioned earlier, composite materials are a combination of fibers and resins. Information on various types of fibers and matrix materials used in polymer matric composites (PMC) is highlighted below:

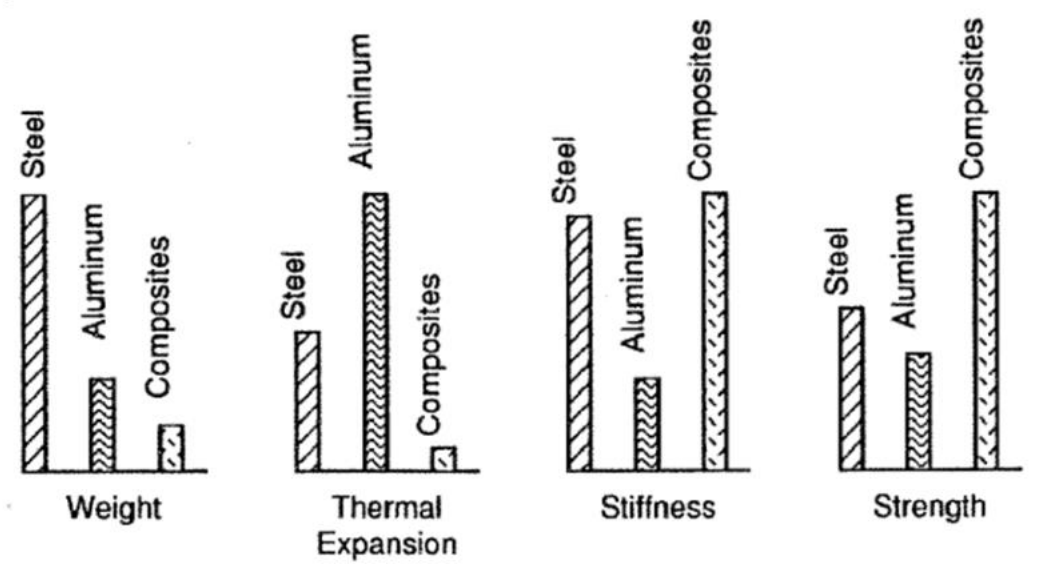

Figure 1.2: Comparison of conventional materials to that of composite materials [35]. (Courtesy of General Dynamics, Convair Division)

\subsubsection{Classification of Composites}

Fiber materials involved in structural applications are utilized as reinforcement as they provide strength to the material [36] and are typically manufactured with numerous types. Carbon fibers which are a family of Poly Acrylic Nitrile (PAN), pitch based fibers, boron fibers, silicon carbide fibers, aramid fibers and alumina fibers are few fiber materials that are commonly used. Various dry fiber forms such as rovings, tows, yarns, non-woven and woven fabrics, braided fabrics, non-crimp fabrics and tapes are also utilized as typical fiber materials. There also exists three dimensional textile preforms which are a subclass of a dry fiber form where the reinforcement is manufactured as a single product [34]. 
Matrix materials shares and transfers the load in and out from the fibers while offering rupture resistance to fibers. The contribution of the matrix phase in a composite is significant and could affect the mechanical performance of the composite. The matrix phase also serves as a protection layer for fibers and qualifies to provide resistance from severe environments operating on an aircraft. Several thermosetting resins such as epoxy resins, polyester resins, vinyl-ester resins, phenolic resins, bismaleimide resins, polyimide resins and cyanate resins are typically utilized for high temperature applications. For low temperature applications, many thermoplastic resins that are amorphous or semi-crystalline thermoplastics such as polyketones, polyphenylene sulfide, polysulfone and polyetherimide are typically used [34].

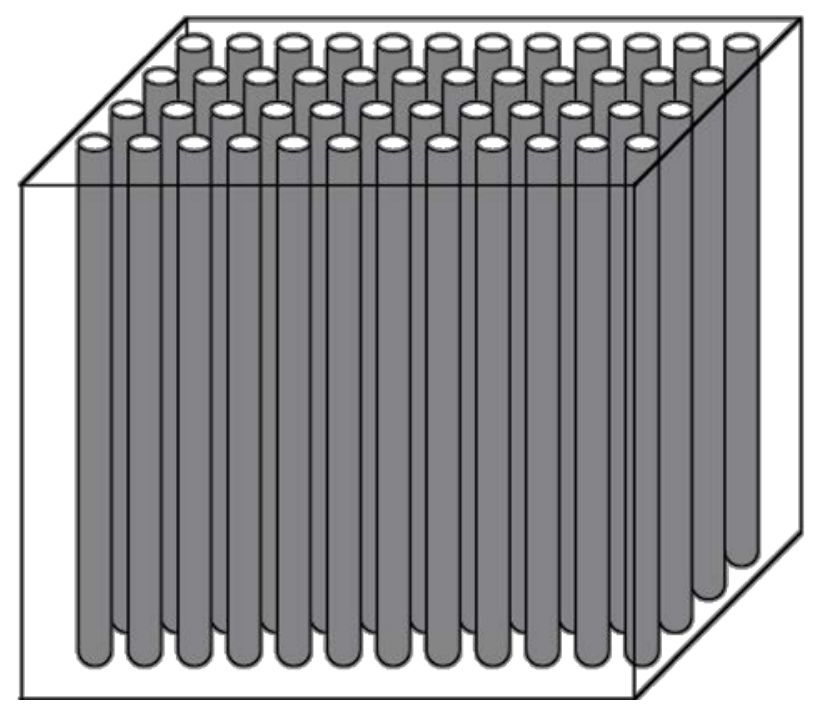

Figure 1.3: Schematic of a Unidirectional Fiber Composite [37].

Unidirectional Fiber Reinforced Composite: Any fiber-reinforced composite with all the fibers aligned in a single direction are termed as Unidirectional Fiber Reinforced Composites. These may be used in a structural member that can carry unidirectional loads where the ability to carry a tensile load is greatest in the fiber direction [33]. Consequently the tensile strength in this fiber direction will be of prime importance in the design of such structural members. A schematic of a unidirectional fiber composite is showed above in Figure 1.3.

Carbon fiber composites with the aforementioned qualities can be recognized as future 
materials that draw tremendous attention and discussion among chemists, polymer and material scientists in perceiving their imminent advancements. However, the production of such high technical materials is often expensive and only limits itself to top-tier applications. It is estimated that by the year 2020, the demand for CFRPs will reach $120 \mathrm{~K}$ tons/year, which is a significant increase from the current usage of 60Ktons/year [38, 39] as shown in Figure 1.4. This increase is expected to play a major role in its application in a wide variety of areas including energy, environment, safety, military, aerospace and sports [38].

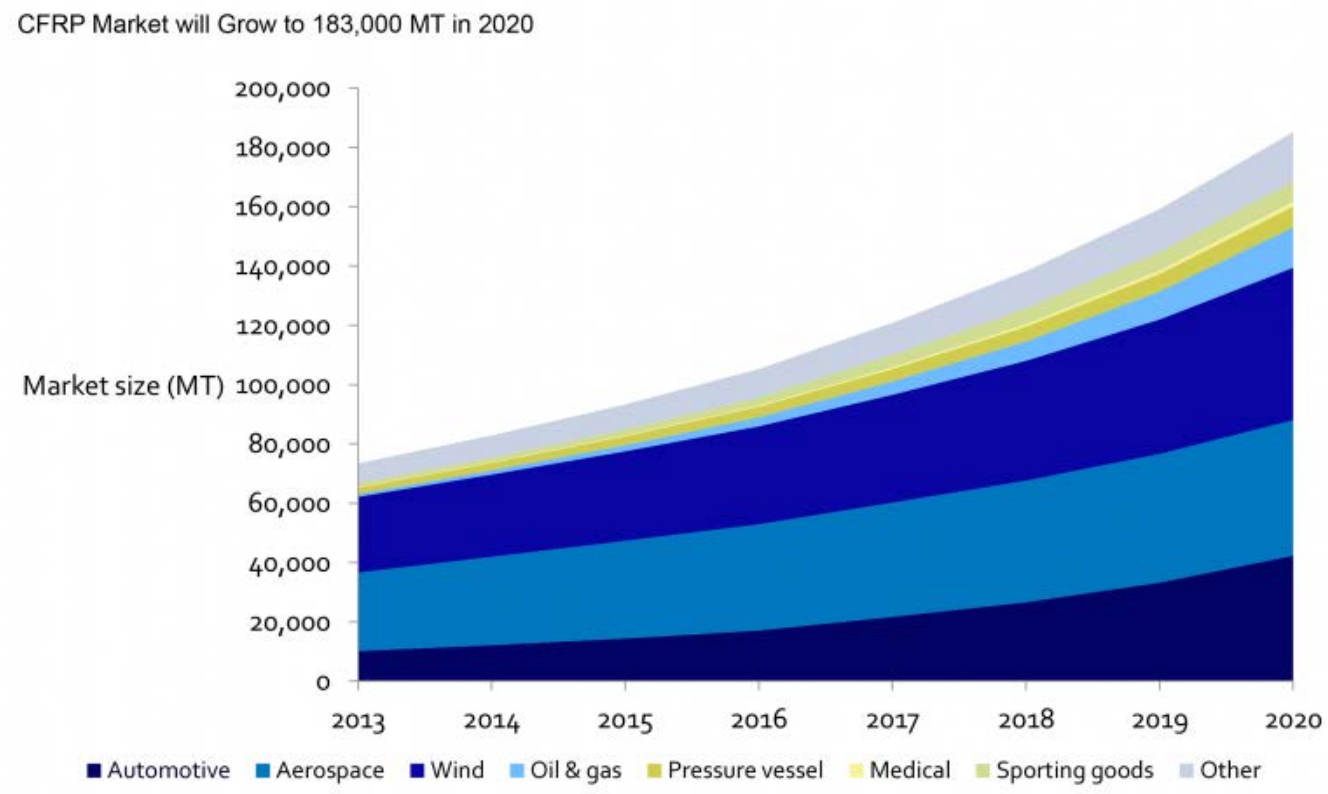

Figure 1.4: Market growth of CFRP and its predictions in various domains [40].

\subsubsection{Economic aspects of Composites and their commercial products:}

Carbon fiber composites, known for their high strength and rigidity, have changed the predilection of consumers and manufacturers. Lux Research Inc, lays out an in-depth market survey of the usage of CFRP and its needs for current as well as near term production till 2020. A 13\% compounds annual growth rate is expected from the year 2013 to 2020 with production values ranging from 36000 tons to 86000 tons respectively [40]. This equates to $\$ 15.4$ billion in 2013 to $\$ 35$ billion in 2020 as mentioned in Figure 1.5. Amongst the world production of CFRP materials, 
Europe is the fastest growing carbon fiber market due to an expanding trade in that region while the North America dominates the largest market share in global CFRP market where aerospace and defense industries are the two major sectors [41].

CFRP Market will Grow to $\$ 35$ Billion in 2020

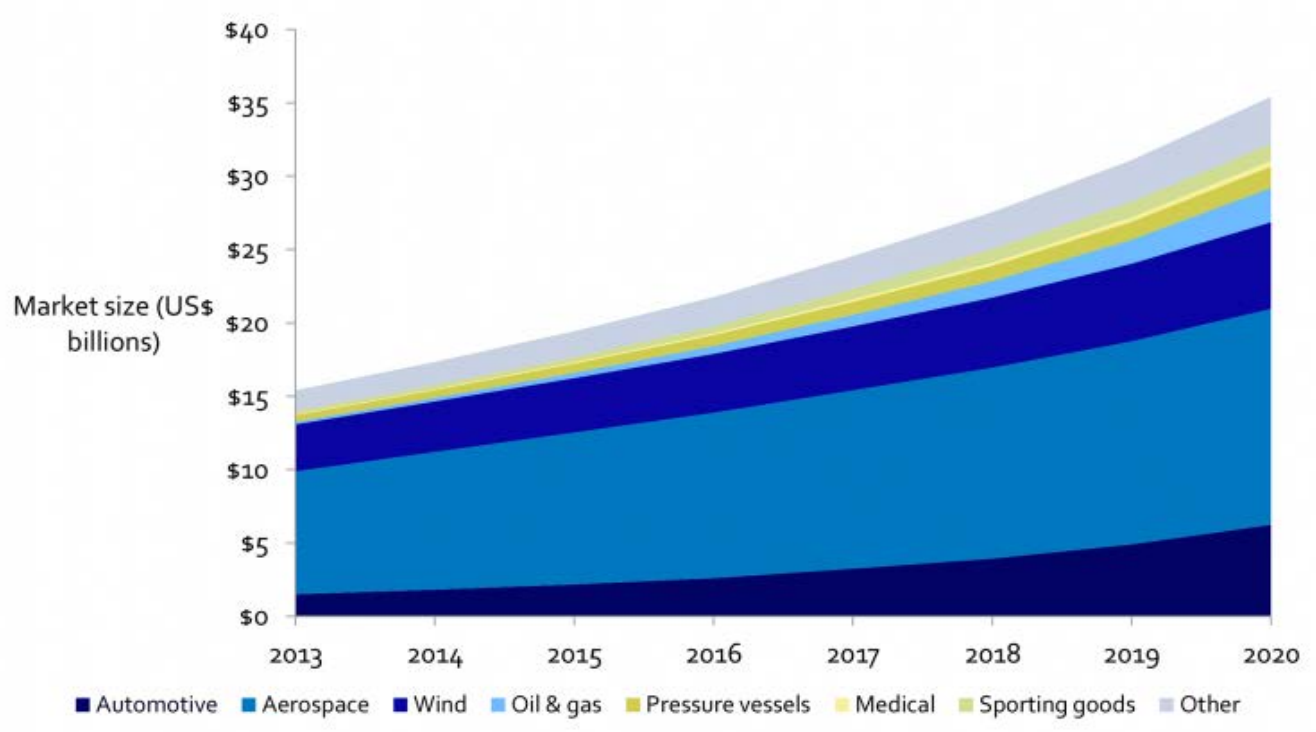

Figure 1.5: Compound Annual Growth Rate (CAGR) of CFRP market and its predictions [40].

Carbon fiber or CFRP’s primary industries include Aerospace \& Defense, Automobile, Marine, Sports Equipment, Wind Energy and Civil, of which the most promising sector being the aviation, wind farming and motor vehicles [32, 41, 42, 38]. Some of the leading players of carbon fiber and CFRP market industry are: Toray Industries (Japan), SGL-Carbon(Germany), Kringlan Composites (Switzerland), Cytec Industries (U.S.), Plasan Carbon Composites (U.S.), Teijin Ltd (Japan), Hexcel Corporation (U.S.), Mitsubishi Rayon (Japan), Hyosung Corp (South Korea), Formosa Plastic Corporation (Taiwan), Gurit Holdings (Switzerland) and Tencate (The Netherlands) [43].

Over time, aerospace structures have assisted in building confidence in composites technology, with the next-generation aircrafts such as Airbus A380 and Boeing 777 have about 50 percent composite content when compared with $8 \%$ for the previous generation of aircraft [42] as 
shown in Figure 1.6. Current composite materials possess more rigid fibers, better ductile resins and are resistant to various harsh conditions. These materials have established themselves in the construction of structural components such as wings, fuselage and substructures.

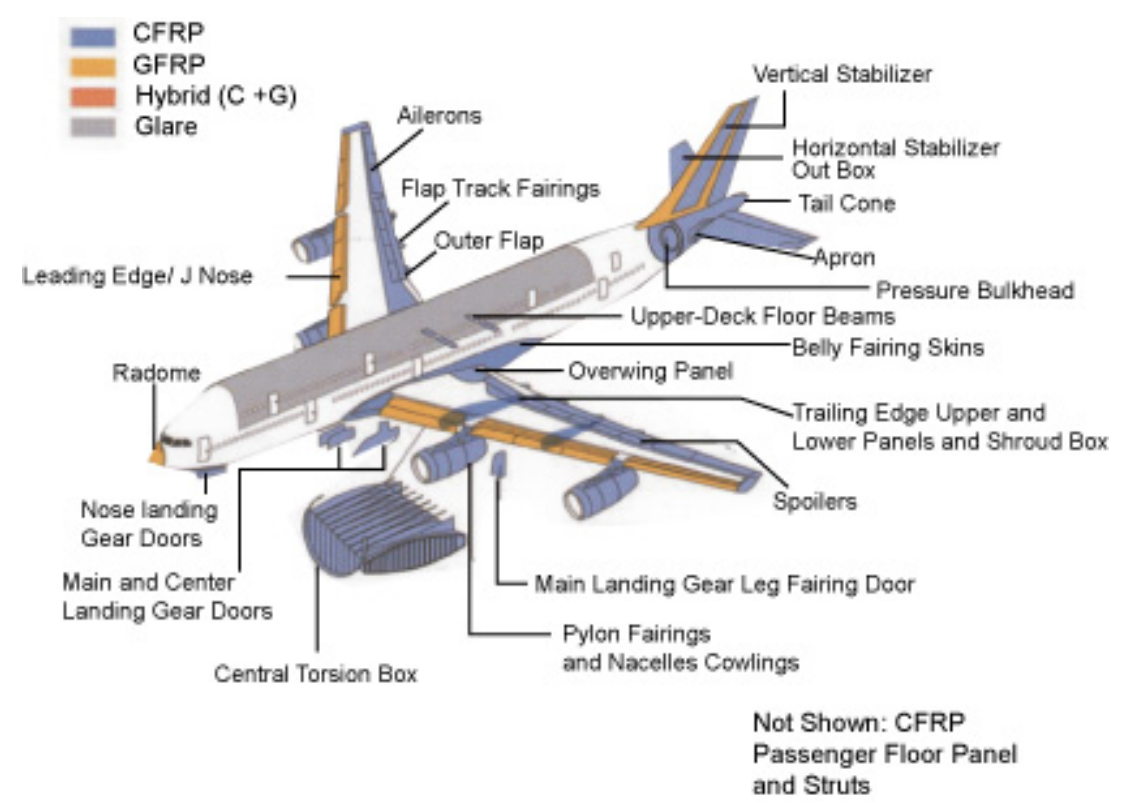

Figure 1.6: CFRP constituting majority of Airbus A380 aircraft structure [44].

\subsubsection{Adhesives}

A joining technique used to bond two dissimilar or identical materials that are fastened producing a close contact between the two materials. The joining technique could be a physical bond or a chemical bond depending on the adherend and the adhesive. Adhesives (synthetic adhesives) have been developed as early as 1900’s [45] and their evolution over time has been very progressive [46]. Conventional joining, however, does exist where nails, rivets, screws etc. are utilized to join the materials which generates a localized stress concentration, potentially becoming a primary source of failure. Most of the current engineering joining applications lean towards using adhesives, as they aid in efficiently transferring the load from the joining materials to the adhesive. The development of new materials has greatly improved their usage in a multi domain industry such as automotive, aerospace, sporting goods, wind technologies etc. Typical adhesives can be 
utilized to bond substrates such as ceramics, metals or even polymers. For highly advanced aerospace applications, lighter weight materials such as composites can often be used with adhesive bonding due to their uniform stress distribution in the joint. This allows for full utilization of the strength and rigidity of the adherends [47]. The localized stress concentrations are eliminated significantly due to the uniformity in the stress distribution across the load bearing part i.e. adhesive.

\subsubsection{Classification of Adhesives}

Adhesive bonding is primarily classified into structural and nonstructural adhesive bonding, based on the numerous emerging bonding technologies in the current market. Adherends which encounter large stresses equivalent to their yield point are termed structural adhesives. These bonds are capable of transferring loads and stresses without compromising the integrity of the design limits [47]. They also possess shear strengths greater than several orders of magnitude of a typical bond and produce a uniform bondline thickness. Additionally, the durability of such bonds are long lasting and highly resistant to aging. Film adhesives usually fall in this category. On the contrary, nonstructural adhesives such as paste adhesives, pressure sensitive adhesives (PSA) and packaging adhesives impose limitations such as non-uniformity in the bondline produce inconsistencies in load transfer mechanisms, however they maintain a considerable integrity of the adhesive and the adherend. An example of a paste adhesive bearing limitations is the super glues which are dominant with cyanoacrylates and possess a poor resistance to moisture and heat [48]. With the development of synthetic polymeric materials having superior mechanical properties, the major usage of adhesives increased over decades of time in a wide variety of domains.

Commercially available structural adhesives typically contain a dicyandiamide curative apart from various other proprietary additives including, but not limited to tackifiers, plasticizers, flow modifiers, fillers, colorants, meshes/scrims, neutralizing agents, stabilizers and antioxidants etc. These additives, when incorporated in several compositions with weight percentages (wt \%) as 
low as 0.05 to approximately 25, helps in attaining properties for a desired application. It should be noted that the additive percentage levels are truly based on the total weight of the epoxide composition used for the resin type based on the application[49]. Additionally, there exist polyurethane adhesives, epoxy adhesives, UV curable adhesives, toughened acrylics, methacrylate adhesives and cyanoacrylate adhesives to name a few, that are also utilized based on the type of application.

\subsubsection{Economic aspects of Adhesives and Commercial Products}

Adhesive materials, particularly structural adhesives known for their high strength and moisture resistant properties have changed the preference of bonding technologies, their consumers and manufacturers. The global market value of aerospace adhesives was estimated to be $\$ 1550.71$ million in 2013 and was projected to be $\$ 2189.80$ million by 2019 [50]. This accounts to a 5\% compound annual growth rate where the major driver being the aircraft passengers, deliveries and military usage [51]. As mentioned in section 1.2.2.1 about several types of adhesives, Figure 1.7 illustrates the structural adhesives market share from the year 2012 to 2022. Commercial products of structural adhesives include aerospace structures such as helicopter rotor blades, full aluminum vehicle bodies, composite roof bonding, bonded seat structures, engine mounts, bushings, transmission systems, trunks, spoilers, cladding, composite panels etc. Some of the leading worldwide adhesives and sealant manufacturers include 3M (USA), Henkel AG \& Co. KGaA (Germany), Huntsman (USA), Illinois Tool Works Inc (USA), Avery Dennison (USA), Sika AG (Switzerland), Dow Corning Corp. (USA), MACtac (USA), Momentive Specialty Chemicals Inc. (USA), RPM International Inc. (USA), MAPEI (USA), Bostik SA (France), H.B.Fuller (USA) etc. 


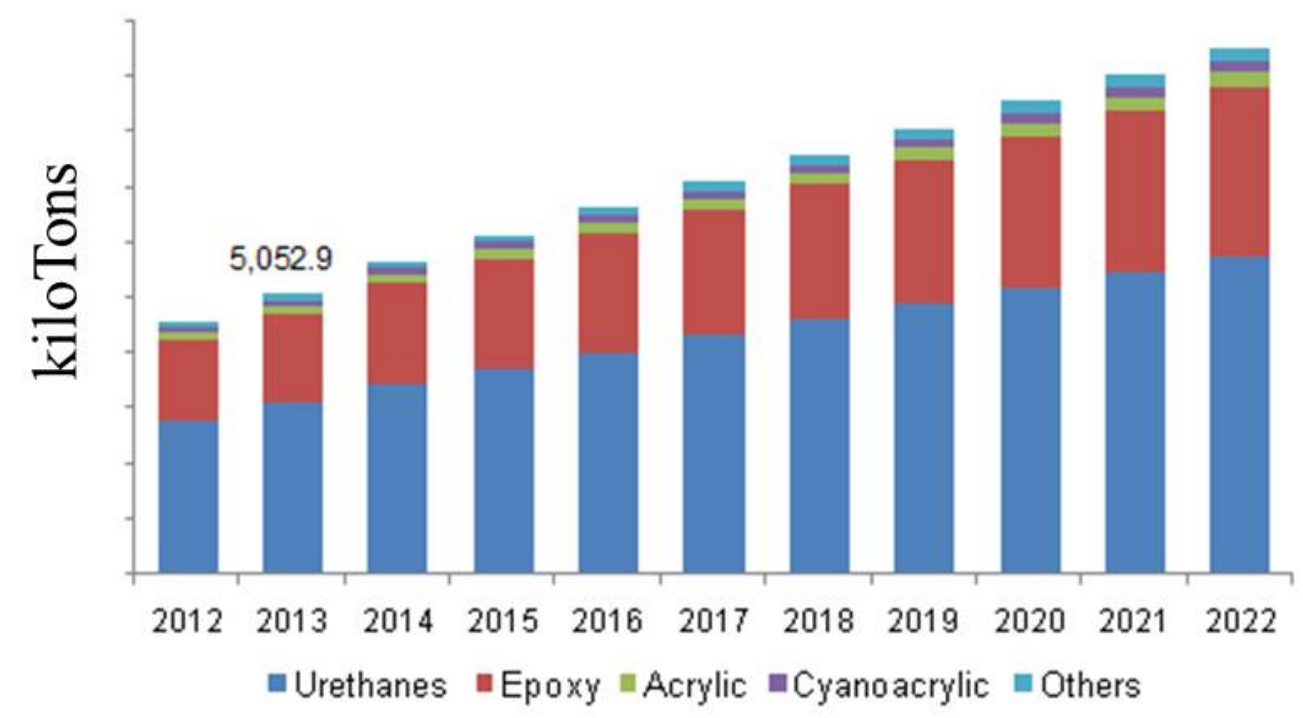

Figure 1.7: Global Structural adhesives market share by product, 2012-2022 [52].

\subsection{Background on Adhesive bonding}

Ever increasing demands from both customers and safety regulators have resulted in promoting bonding technologies where the weight savings is a key driving force. Bonded structures typically include metal-to-metal, composite-to-metal and composite-to-composite bonding with the aid of a highly efficient structural adhesive. ABCJ's are expected to sustain static and cyclic loads for extended periods of time without having negative effects on the load bearing capacity of the structure [14]. Currently, adhesive bonding has been limited to secondary bonded structures, i.e., composite repair, single or double sided doubler patches [53], scarf or stepped scarf patches [54, 55, 56] that are adhesively bonded to a damaged site. Repair methodologies typically fall under secondary bonding where the composite laminates are precured and are bonded with the help of adhesives. In contrast, co-curing techniques are utilized as a joining technique where the composite laminate and the adhesive are cured together $[57,58,59]$. In aircraft construction, a well-designed adhesive bond can actually result in a stronger structure and can provide higher ultimate strength than many metals [15] which is a huge advantage with bonded structures. Figure 1.8 shows the adoption of adhesive bonding technique in manufacturing an aerostructure member. Thus, there is 
a push to increase structural efficiency by promoting adhesive bonding [11] as primary joining technique in today's aircraft transportation market.

Under the patronage of the U.S. Department of Defense (DOD) funded, the then Primary Adhesively Bonded Structure Technology (PABST) program to its most recent Composites

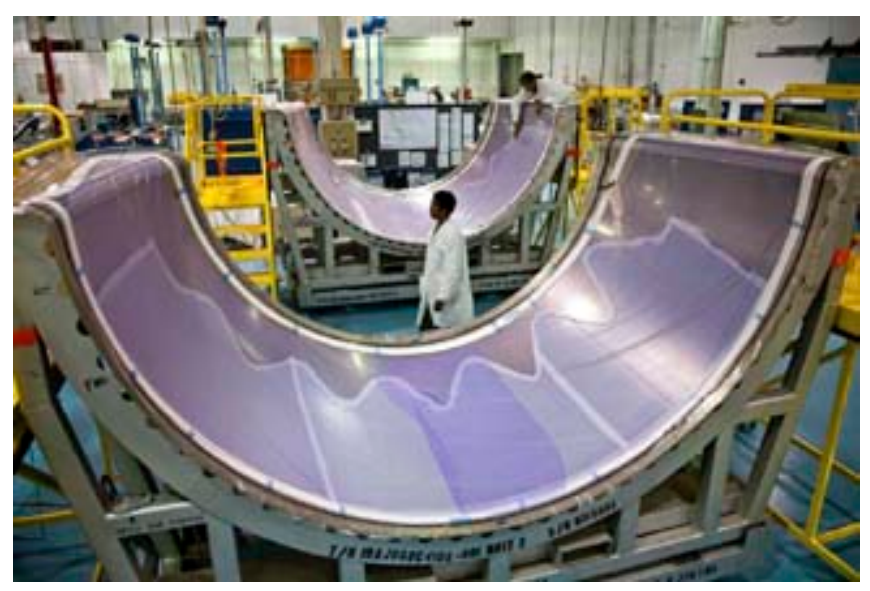

Figure 1.8: Bonding fabrication process of aerostructures [61]

Affordability Initiative (CAI) program, considerable research efforts are being undertaken to emphasize the need for increasing the structural efficiency of primary aircraft structures [57]. This advanced in conjunction with the European Union (EU) funded Boltless assembling Of Primary Aerospace Composite Structures (BOPACS) program elaborating the worthiness of adhesive bonding in aircraft structures. Therefore directing the need for investigations on the strength and durability of these bonded structures, that is of paramount importance to the manufacturers and consumers. Of particular interest is the short term strength and long term durability of these adhesively bonded systems that offer reliability in the bonding process and qualifies for the necessary certification requirements.

\subsubsection{Adhesive Bond Strength}

A general adhesive bond is shown in Figure 1.9. The primary way to measure the quality of a bond is to fail the bond which can lead to a quantification of the toughness of the bond i.e. the resistance of the adhesive to the propagation of a crack. Bond strength is usually conceptualized 
based on the loading conditions a bond experiences under tension or compression and is classified as a good or bad bond as shown in Figure 1.10. Failures are governed by the type of loading and considering that the adhesive bond is loaded in tension i.e. the applied force on the adherends is in a direction perpendicular to the bond, the mode of failure is determined.

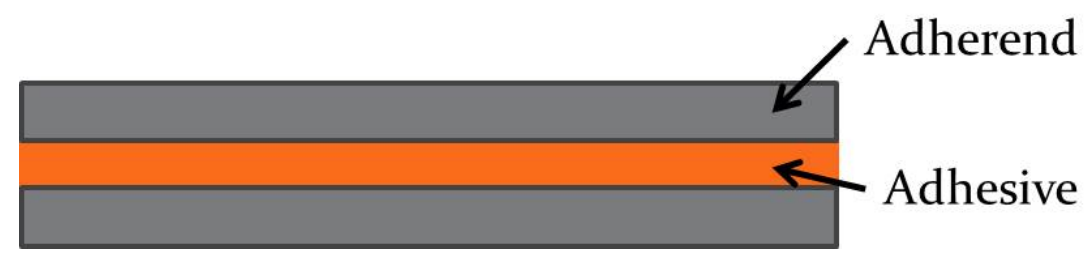

Figure 1.9: A typical bonded joint showing adherends bonded with adhesive

A failure is determined to be an adhesion failure, if the bond fails between the adhesive layer and one of the adherends as shown in Figure 1.10a. This also depicts that the bond is a pure physical bond and exhibits an interlocking mechanism than crosslinking. Figure 1.10b shows a failure occurring in the adhesive layer with the adhesive on both sides of the adherends. This is called a cohesion failure and the nature of the bond is termed acceptable as the adhesive crosslinks with the adherend and the failure occurs at the weakest site within adhesive. Figure 1.10c shows the failure in the adherend which can be termed as interlaminar failure i.e. the strength of the bond surpasses that of adherend, but it is also a bad joint design. A failure is initiated in the bond depending on the mode of loading. The fracture is subjected to occur in a definitive pattern dictating the type of failure that has occurred within the bond.

Figure 1.11 displays the three modes of loading which are used to evaluate the fracture toughness of a joint. Each of the three modes require monitoring of the crack and the failure patterns. Mode I is called a tensile or opening mode where the loads are applied perpendicular to the joint as shown in Figure 1.11a. Mode II is called the sliding or shear mode where the load is applied inwards to the plane causing it to shear as shown in Figure 1.11b. Mode III, shown in 
Figure 1.11c, is termed a tearing shear or out- of- plane shear mode as the load is applied out of the plane parallel to the

a) Bad Bond/ Adhesive failure

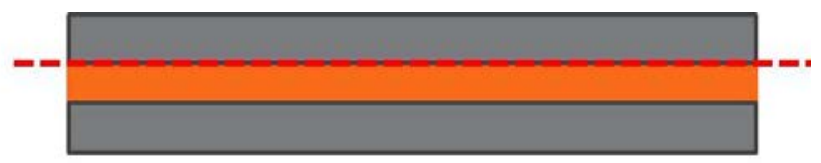

b) Good Bond/Cohesive failure

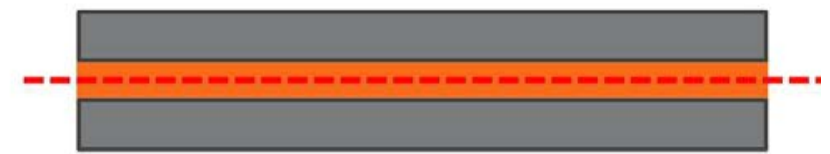

Good Bond

c) Bad Joint Design

Interlaminar failure

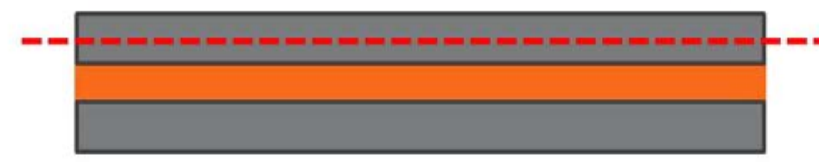

Figure 1.10: Typical bond failure occurrences dictating the type of failure a) adhesive failure; b) cohesive failure; c) interlaminar failure

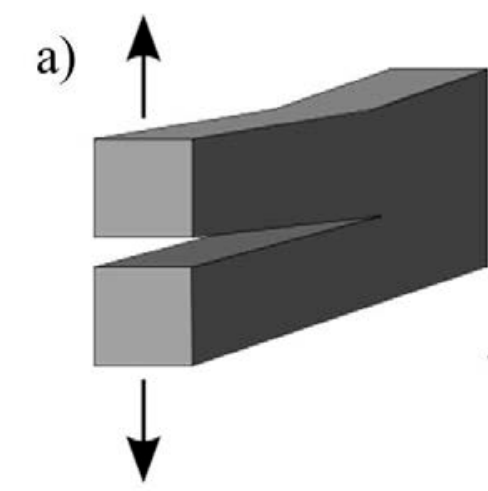

Mode 1:

Opening or Tensile mode
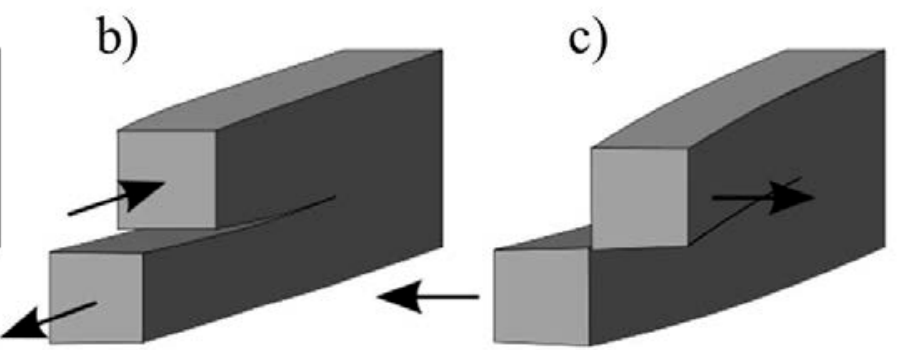

Mode II:

In-plane Shearing mode
Mode III:

Out-of-plane Shearing mode

Figure 1.11: Crack Opening Modes

beam. In conventional engineering practice, Mode I is proven to be more useful as it examines the stress state near the tip of a sharp crack and the smallest strain energy release rates can be evaluated only through Mode I. 


\subsubsection{Mechanisms of Adhesive Bonding}

In order to understand the mechanisms of adhesion, it is appropriate to understand the underlying theories of adhesion and relate them to the bond strength or to the physical properties of adhesives. Many theories have been postulated that correlates well with the adhesion mechanisms such as mechanical interlocking, electrostatic theories, diffusion and adsorption/ surface reaction theories. A good understanding of the above mentioned theories will help in discerning the adhesion mechanisms between the adhesive and adherend.

\subsubsection{Mechanical Interlocking}

With mechanical interlocking, adhesives flow into the irregularities or the surface ridges of the adherend. The surface ridges can exist in the form of pores, cavities or channels which is attributed to the surface roughness. This creates an interlocking effect as shown in Figure 1.12, for which the crack propagation path becomes tortuous and becomes difficult to grow. However, a surface with higher roughness is not always feasible for better adhesion [61] as well as surfaces with lower roughness or a smooth surface tends to propagate a crack very easily.

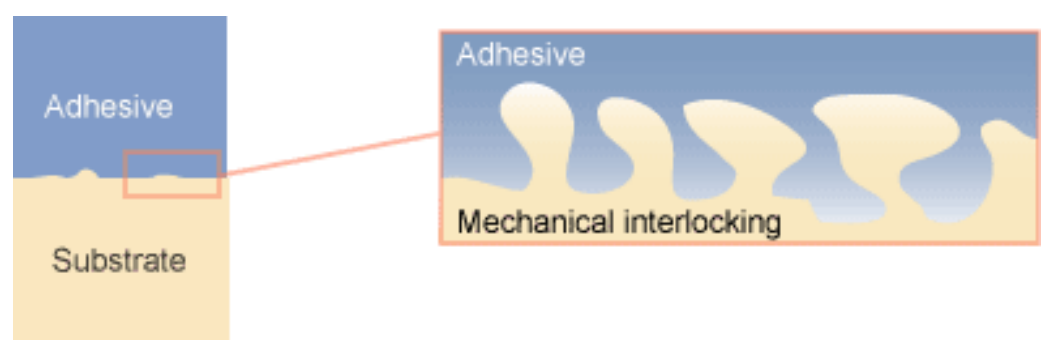

Figure 1.12: Schematic showing the interlocking effect of the adhesive into the adherend.

\subsubsection{Electrostatic Theory}

In electrostatic theory, the adhesive and adherend have different electronic band structures through which the adhesive and adherend transfer charge i.e. an electropositive material donates charge to electronegative material as shown in Figure 1.13. This theory is valid for metallic bonds, however, the electronic transfer in polymeric systems has found to be relatively small in comparison [62]. 


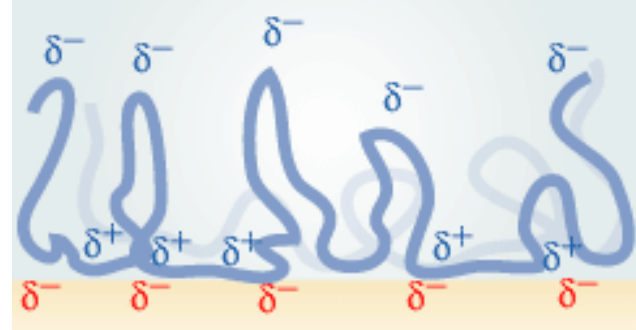

Figure 1.13: Schematic showing the charge transfer mechanism between adhesive and adherend.

\subsubsection{Diffusion Theory}

This theory states that the adhesion phenomenon is due to the interdiffusion of molecules in between the adhesive and the adherend where the interaction between the polymeric chains at the interface is prominent. Since the polymeric materials are high in their molecular weight, their relatively long-chain molecular structures help in their movement and crosslink at bonding conditions as shown in Figure 1.14. The crosslinking density or the entanglement of the polymeric chains depends on the curing time, temperature chemical as well as physical forms.

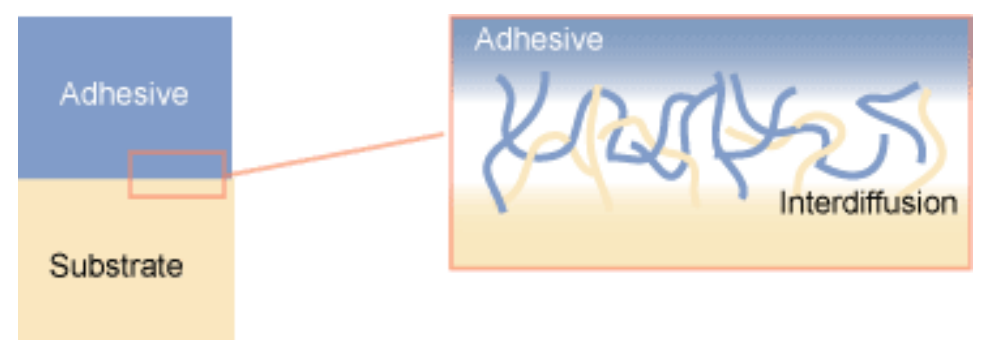

Figure 1.14: Schematic showing the interdiffusion of adhesive and adherend.

\subsubsection{Wetting Theory}

According to the wetting or adsorption theories, adhesion between an adhesive and adherend results from molecular contact i.e. interatomic forces between the atoms and molecules on the respective surfaces interact continuously, largely Vander Waals forces. Figure 1.15 illustrates the wetting mechanisms where it can be inferred that optimal wetting results when the adhesive flows into the hills and valleys of the adherend, resulting in good bond strength. It should 
be noted that adhesive bonding cannot be attributed fully to one particular mechanism but typically occurs as a combination of several mechanisms.

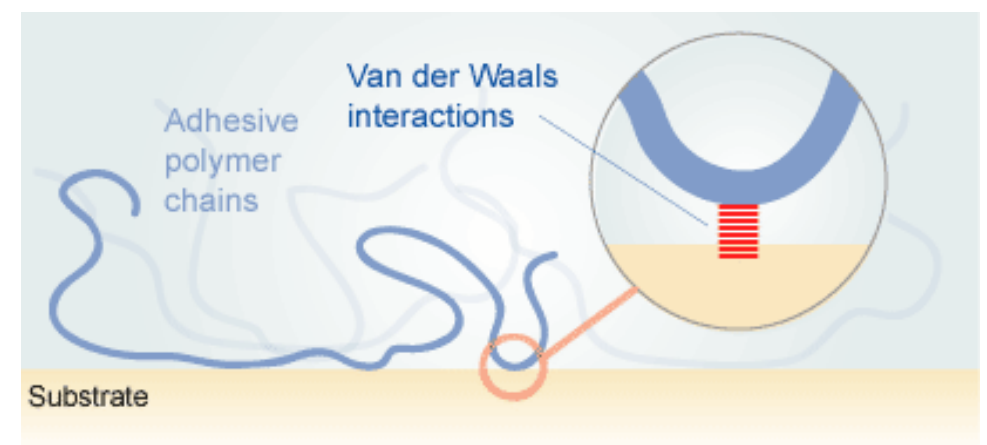

Figure 1.15: Schematic showing polymeric chains interacting with the substrate i.e. Vander Waals interaction.

\subsection{Manufacturing of bonded composite joints}

The structural components used in an aircraft specifically with composites are often directional dependent and could be unidirectional, bi-directional and tri-directional weaved fibers with the embedding matrix phase [34]. Below, manufacturing terms typically used in the fabrication of composite laminates and their bonding is provided.

Prepreg: A ready-to-mold or cure material in sheet form where the fibers are embedded at a certain volume percentage in a matric (usually epoxy). This may be tow, tape, cloth or mat impregnated with resin. Storage is always in freezing temperature as the resin may tend to react at ambient temperature. The shelf life is always preserved by keeping the length of the ambient exposure time minimal.

Peel Ply: A peel ply material is a cloth used to protect the top surface layer of a composite laminate that is to be bonded. It is co-cured with the laminate during the primary cure on the top most surface layer and does not contain transferable chemical release agents. Its easy peel off nature depends on type of cloth and the release agents embedded within the ply material as it is intended to completely remove itself from the laminate prior to subsequent bonding process. The pull-out/removal process can be troublesome as it leaves the surface with variable surface ridges which favorable or un- 
favorable for adhesion, but the surface is clean and free of contaminants with a highly textured resin fracture surface leaving a weaved pattern.

Layup: A process to stack a measured lamina (a single ply) in specified sequence and orientation while building up their successive laminae (series of plies) on top of each other in desired orientation.

Release Ply/Film: A specific film that is placed between the tool plate and the stacked composite laminae, in order to prevent the adhesion between epoxy and the tool plate during the cure cycle. With the help of the release film, it's always easy to remove the cured laminate due to the presence of a non-reactive high temperature polytetrafluoroethylene (PTFE) or its derivative which doesn't participate in the cure reaction with the overlaying composite laminate, which also leaves a smooth surface finish with minimal chemical residue on the cured component.

Breather/Bleeder cloth: A specific foamy cloth which is non-woven that allows air and volatiles to be removed from within the vacuum bag throughout the cure cycle. They are also utilized to absorb the excess resin that bleeds out of the composite lay-ups without risk of bridging.

Vacuum Bag Molding: A process in which the stacked composite laminae is cured under pressure generated by drawing a vacuum in the space between the layup and a flexible vacuum sheet placed over it and sealed at the edges using a high temperature sticky tape.

Autoclave Molding: A baking process where pressure and heat are applied at the same time to cure a specific material. The specimens are pressurized using the pressure while application of heat allows curing the specimens (initiates reaction). The output of the process renders a uniform quality as the pressure and heat are distributed precisely. Simple and complex geometries can be achieved using this technique.

Primary curing: The joining of layered composite parts for the first time where the only chemical reaction occurring is the crosslinking of the layered laminae. 
Secondary curing: The joining of two or more already cured composite parts, during which the only chemical or thermal reaction occurring is the curing of adhesive itself.

Co-curing: When both primary and secondary cures take place simultaneously i.e. curing a composite laminate and bonding together during the same cure cycle.

\subsection{Overview of this study}

This chapter focuses on providing the necessary background information related to composites and adhesive bonding, their progress over the period of time, demonstrating their classification and economic impact. Many of the insights presented in this chapter are focused on adhesive bonding of composite joints where some of the background information on structural adhesive bonding was provided. Best practice in development and substantiation of adhesive bonded structures primarily requires a good understanding of the theories associated with adhesive bonding along with conceptualizing bond strength. The reliability of a bond is indicative of its performance and hence thorough understandings of bond failures were theoretically demonstrated. The difficulty in the acceptance of a bonded structure is challenged when the service life of the bond is considered i.e. the long term durability of these adhesively bonded systems. Issues associated with the durability and related failures in aircrafts, continue to be a critical hindrance to the universal acceptance of ABCJs $[15,16]$. Numerous studies have been conducted to determine the initial bond strength of ABCJs, yet there is still limited understanding on the assessment of their long term strength. The extrinsic factors that affect the strength and durability of ABCJs are discussed in detail in Chapter 2. 


\subsection{Contributions}

This investigation will advance the knowledge and understanding of adhesively bonded composite systems and a number of major concerns associated with ABCJ's for their adoption in the aircraft industry as primary joining technique. This would be achieved via the following:

- $\quad$ The development of a contamination procedure, that not only is scalable and reliable but also repeatable, providing insight to the effects of undesirable bonding conditions on short and long term bond strength of ABCJ's. This process can serve as NDE benchmark for detecting contamination or weak bonds in ABCJ's.

- $\quad$ The development of a test methodology to access/evaluate the durability of ABCJ's (the elucidation of bond durability under elevated temperatures and humidity over extended period of time) helps in understanding their long term performance, serving as reliable basis for bonded joints and to their adoption in primary structural components.

- $\quad$ Quantification of the failure ratios through image processing technique that resulted in a direct correlation of the fracture property evaluated experimentally.

- $\quad$ Microscale testing helped in understanding the initiation and damage mechanisms that correlated well with macroscale testing providing an insight in understanding the fracture behavior of ABCJ's with undesirable bonding conditions.

- $\quad$ Correlating the bond strength with the theoretical model that assisted in predicting the fracture toughness of a bonded joint with undesirable bonding conditions. 


\subsection{References}

[1] International Air Transport Association (IATA), "New IATA Passenger Forecast Reveals Fast Growing Markets of the Future," Corporate Communications, 16 October 2014.

[2] R. Cannegieter, "Long Range vs. Ultra High Capacity: "Ranging Capabilities..."," Aerlines Magazine e-zine edition, 2011.

[3] J. Max Kingley, "Boeing's 747-8 vs A380: A titanic tussle," Flightglobal, Toulouse, 17 February 2006.

[4] Chapter 5, "Aircraft Materials, Processes \& Hardware," in Aviation Maintenance Technician Handbook, Oklahoma, OK: U.S. DOT Federal Aviation Administration, 2008, pp. 1-84.

[5] W. G. Roeseler, B. Sarh and M. U. Kismarton, "Composite Structures: The First 100 Years," in 16th International Conference on Composite Materials, Kyoto, Japan, 2007.

[6] National Research Council, "New Materials for Next Generation Commercial Transports," National Academy Press, Washington, D.C., 1996.

[7] C. Peel, "Advances in materials for aerospace.," The Aeronautical Journal of the Royal Aeronautical Society, pp. 487-503, Dec 1996.

[8] P. Mangalgiri, "Composite materials for aerospace applications," Bulletin of Materials Science, vol. 22, no. 3, pp. 657-664, May 1999.

[9] E. Barbero, Introduction to Composite Materials Design, Boca Raton, FL: CRC Press, 2011.

[10] R. Dickie, S. Labana and R. Bauer, "Chemistry, Properties and Applications," in CrossLinked Polymers, American Chemical Society: Div of Poly Mat:Sci. \& Engg., 1988.

[11] B. Jang, Advanced Polymer Composites: Principles and Applications, ASM International, 1994.

[12] H. Lee and K. Neville, Handbook of Epoxy Resins, McGraw-Hill, 1967.

[13] G. Kelly, Joining of Carbon Fibre Reinforced Plastics for Automobile Applications, Stockholm: Royal Institute of Technology, 2004.

[14] M. Banea and L. da Silva, "Adhesively bonded joints in composite materials: An overview," Journal of Materials Design and Applications, vol. 223, no. 1, 2009. 
[15] M. Davis and D. Bond, "Principles and Practices of Adhesive Bonded Structural Joints and Repairs," International Journal of Adhesion and Adhesives, vol. 19, pp. 19-105, 1999.

[16] T. Pribanic, D. McDaniel, V. Musaramthota, L. Sanchez, N. Munroe, X. Zhou, J. Zhou and S. Cai, "Development of Durability Test Procedure for Adhesively Bonded Composite Joints," in SAMPE, Baltimore, MD, 2012.

[17] S. Xu, D. Dillard and G. Dillard, "Environmental aging effects on the durability of electrically conducive adhesive joints," International Journal of Adhesion and Adhesives, vol. 23, pp. 235-250, 2003.

[18] B. Parker, "The effect of extended hot-humid exposure of adhesive bonded carbon-fiber composite joints. Part 1: 175 C cured matrix," RAE Technical Report 89001, January 1989.

[19] and B. Parker, "The effect of extended hot-humid exposure of adhesive bonded carbon-fiber composite joints. Part II: 120 C cured matrix," RAE Technical Report 89061, January 1990.

[20] N. Datla, M. Papini, J. Ulicny, B. Carlson and J. Spelt, "The effects of test temperature and humidity on the mixed-mode fatigue behavior of a toughened adhesive aluminum joint," Engineering Fracture Mechanics, vol. 78, no. 6, April 2011.

[21] H. Kim, C. Sun and T. Siegmund, "Damage Tolerance and Durability of Adhesively Bonded Composite Structures," in Advanced Materials in Transport Aircraft Structures (AMTAS), April 2007.

[22] J. Bardis and K. Kedward, "Effects of Surface Preparation on the Long-Term Durability of Adhesively Bonded Composite Joints," U.S. Department of Transportation, 2004.

[23] L. Hart-Smith, "A Peel-Type Durability Test Coupon to Asses Interfaces in Bonded, CoBonded, and Co-Cured Composite Structures," International Journal of Adhesion and Adhesives, vol. 19, pp. 181-191, 1999.

[24] D. Klapprott and J. Tomblin, "Best Practice in Adhesive Bonded Structures and Repairs," U.S. Department of Transportation, April 2007.

[25] D. Klapprott, H. Li, R. Wong and G. Geisendorfer, "Key factors of the Peel ply surface preparation process," Henkel Corporation, Long Beach, CA.

[26] G. Dillingham, B. Oakley, P. Van Voast, P. Shelley, R. Blakley and C. Smith, "Quantitative Detection of Peel Ply Derived Contaminants via Wettability Measurements," Journal of Adhesion Science and Technology, vol. 26, pp. 1563-1571 , 2012. 
[27] L. Hart-Smith, G. Redmond and M. Davis, "The Curse of the Nylon Peel Ply," in $41^{\text {st }}$ International SAMPE Symposium, 1996.

[28] P. Van Voast, P. Shelley, R. Blakley, C. Smith, M. Jones, A. Tracey, B. Flinn, G. Dillingham and B. Oakley, "Effect of Varying Levels of Peel Ply Contamination on Adhesion Threshold," in Society for Advanced Materials and Processing (SAMPE), Seattle, WA., 2010 .

[29] A. Baker, "Development and Potential of Advanced Fibre Composites for Aerospace Applications," Materials Forum, vol. 11, pp. 217-231, 1988.

[30] J. Raghavan, M. Meshi and D. Feng, "Influence of Reinforcing Continuous Carbon Fibers on the Viscoelastic Properties of Epoxy Matrix," Polymer Composites, vol. 18, no. 1, pp. 5563, February 1997.

[31] University of Cambridge, "Materials Information Interactive Charts," Cambridge, UK, 2002.

[32] Composites One LLC, "Fiber-Reinforced Plastics," Arlington Heights, IL, 2010.

[33] S. Aratama, Y. Tsumura, M. Nishikawa and M. Hojo, "Effect of Voids on Microscopic Strain Distribution in CFRP Laminates," in Proceedings of the American Society for Composites 2014-Twenty-Ninth Technical Conference on Composite Materials, La Jolla, CA, 2014.

[34] A. Baker, S. Dutton and D. Kelly, Composite Materials for Aircraft Structures Second Edition, Blacksburg, Virginia: American Institute of Aeronautics and Astonautics, Inc (AIAA), 2004.

[35] Bob Mathews, "Applied Stress Analysis- Composite Materials," General Dynamics, Convair Division.

[36] U.S. Congress, Office of Technology Assessment, "Advanced Materials by Design," U.S.Government Printing Office, Washington, DC, June 1988.

[37] G. C. Foster, Tensile and Flexure Strength of Unidirectional Fiber-Reinforced Composites: Direct Numerical Simulations and Anlytic Models, Blacksburg, Virginia: M.S. Thesis, Engineering Mechanics, Virginia Polytechnic Institute and State University, February 1998.

[38] M. Kamiura, "3rd IT Strategy Seminar on Carbon Fiber Composite Materials," Toray Industries Inc, 11 April 2010.

[39] Mark Holmes (editor), Reinforced Plastics Magazine, "Carbon fibre reinforced plastics market continues growth path (Part 1)," Materials Today, 7 January 2014. 
[40] Anthony Vicari, Advanced Materials, Lux Populi, "The Carbon Fiber Composite Market Will Reach \$35 Billion in 2020, But Still With Unmet Needs for Developers to Target," The Lux Research Analyst Blog, Boston, MA, 25 Oct 2014.

[41] Clare, "Carbon Fiber Market \& Carbon Fiber Reinforced Plastic (CFRP) Market by Type, Fiber size, \& Region; CFRP Market by (Type, Manufacturing processes/semi- finished product, Resin, Application and Region) - Global Forecast to 2019," PR Newswire, New York, October 2014.

[42] F. Monfort-Windels, Sirris, PlusComposites, "Part 4: Carbon fibre reinforced composites," Composites: Materials of the Future, pp. 1-8.

[43] Market Research, Visiongain, "Carbon Fibre Reinforced Polymers (CFRP) Composites Market Forecast 2015-2025: Prospects for Leading Companies," MarketResearch.com, March 2015.

[44] Jim Patterson, FAA Technical Center, "New Large Aircraft Composite Fire Fighting," U.S. DOT, Federal Aviation Administration, Washington, DC, May 2012.

[45] Adhesives and Sealants Council. , "Bonding- An Ancient Art," 14 April 2013.

[46] Bearing Briefs, "History of Adhesives," Bearing Specialists Association, Glen Ellyn, IL.

[47] S. Ebnesajjad, Adhesives Technology Handbook, 2nd Edition, Norwich, NY: William Andrew, 2008.

[48] S. K. Mazumdar, Composites Manufacturing: Materials, Product and Process Engineering, Boca Raton, FL: CRC Press LLC, 27 Dec 2001.

[49] Hebert, Larry S.; Jing, Naiyong; 3M Innovative Properties Company, "Fluoropolymer film with epoxy adhesive," US Patent EP2513204 A1, 24 Oct 2012.

[50] Marketsandmarkets.com, "Aerospace Adhesives \& Sealants Market by Resin Type," Markets and Markets, Washington, US, April 2015.

[51] PR Newswire, "Growth Opportunities for Adhesive in Global Aerospace Industry 20142020: Trend, Forecast, and Opportunity Analysis," PR Newswire, Newyork, 26 May 2015.

[52] Grand View Research, "Structural Adhesives Market Analysis," GVR Market Research \& Consulting, San Francisco, CA, July 2015.

[53] A. Baker, "Bonded composite repair of fatigue cracked primary aircraft structure," Composite Structures, vol. 47, no. 1, pp. 431-443, 1999. 
[54] C. Wang and A. Gunnion, "Optimum shapes of scarf repairs," Composites: Part A Applied Science and Manufacturing, vol. 40, no. 9, pp. 1407-1418, 2009.

[55] A. Gunnion and I. Herszberg, "Parametric study of scarf joints in composite structures," Composite Structures, vol. 75, no. 1, pp. 364-376, 2006.

[56] C. Soutis, D. Duan and P. Goutas, "Compressive behavior of CFRP laminates repaired with adhesively bonded external patches," Composite Structures, vol. 45, no. 4, pp. 289-301, 1999.

[57] K. Shin and J. Lee, "Tensile load bearing capacity of co-cured double lap joints," Journal of Adhesion Science and Technology, vol. 14, pp. 1539-1556, 2000.

[58] J. H. Choi and D. Lee, "Torque capacity of co-cured tubular lap joints," Journal of Composite Materials, vol. 31, pp. 1381-1396, 1997.

[59] C. Cao, Damage and Failure Analysis of Co-cured Fiber Reinforced Composite Joints, Ph.D Dissertation, Georgia Institute of Technology, November 2003.

[60] Triumph Group, Inc, "Bonding Fabrication," Triumph Aerostructures and Vought Aircraft Division, Milledgeville, 2015.

[61] Ginger Gardiner (editor), High Performance Composites, "Certification of bonded composite primary structures," Composites World, Cincinnati, OH, March 2014.

[62] K. Allen, "Some reflections on contemporary views of theories of adhesion," International Journal of Adhesion, vol. 13, no. 2, pp. 67-72, April 1993.

[63] B. Derjaguin, N. Krotova and V. Smilga, "Adhesion of Solids," Journal of Applied Physics, vol. 38, p. 4607, 1967.

[64] A. Kimiaeifar, H. Toft, E. Lund, O. Thomsen and J. Sorensen, "Reliability analysis of adhesive bonded scarf joints," Engineering Structures, vol. 35, pp. 281-287, February 2012.

[65] A. Abdullah, M. Afendi and M. Abdul Majid, "Effect of Adhesive Thickness on Adhesively Bonded T-Joint," in International Conference on Mechanical Engineering Research (ICMER), Pahang, Malaysia, July 2013.

[66] W. Broughton, L. Crocker, M. Gower and R. Shaw, "Assessment of Predictive Analysis for Bonded and Bolted T-Joints," National Physical Laboratory (NPL), West Yorkshire, UK, May 2004. 
[67] L. Crocker and G. Dean, "Tensile Testing of Adhesive Butt Joint Specimens," Crown Copyright, Middlesex, UK, May 2001.

[68] A. Chadegani and R. C. Batra, "Analysis of adhesive-bonded single-lap joint with an interfacial crack and a void," International Journal of Adhesion and Adhesives, vol. 31, no. 6, pp. 455-465, September 2011.

[69] L. Hart-Smith, "Adhesive Bonded Double Lap Joint," National Aeronautics and Space Administration (NASA), Hampton, Virginia, Jan 1973.

[70] A. Kinloch, B. Blackman and W. Teo, "The Adhesive Bonding of Polymeric Matrix Composites," in 17th International Conference on Composite Materials (ICCM), Edinburgh, UK, July 2009. 


\section{LITERATURE REVIEW}

Although some progress has been made recently in the understanding of adhesive bonding, significant efforts were made in the late 80's and early 90's to accommodate aerospace needs. While weight savings was one of the primary thrusts for this research, reduction in component thickness and efficient loading paths were also major drivers. The literature review presented in this chapter provides an overview of the research conducted to date for bonded structures and their durability and is intended to provide rationale for the suggested testing approaches discussed in later sections.

\subsection{Structural adhesives and their chemistry}

Structural adhesives are typically preferred over paste adhesives for bonding of aerospace structures due to their significant cohesive strength and does not exhibit creep under significant design loads. These adhesives are typically doped with filler materials such as alumina and glass to enhance their mechanical properties and electrical and thermal conduction abilities. Due to their specific curing procedures, structural adhesives typically exist in several forms, of which film and paste are the most widely utilized. Film adhesives are comprised of highly crosslinkable proprietary organic compounds that contain curatives necessary to obtain desired bond strength. These adhesives often contain a mesh or a scrim that can alter the mechanical properties of the adhesive layer significantly [1] but provides for uniform bondline thicknesses. Plasticizers are sometime incorporated in special applications where flexibility and elongation are needed. The paste adhesives are usually one or two part systems that require heat or room temperature cure for a complete curing. One part adhesive systems contain all the materials within the adhesive to be cured while the two part system possesses curatives in one part and crosslinkable resins in a second part. Another form of structural adhesives is in the form of liquid curable adhesives, which as the name suggests, are liquid by nature and cures/hardens when exposed to ambient air. There also exist UV curable adhesives where the curing reaction requires UV light to initiate the cure. A wide 
range of structural adhesives are used in industry based on the application need. Examples include Epoxies, Urethane, Phenolic, Polyamides and Acrylic etc [2]. The information on the structural adhesives utilized in this project are proprietary and limited information is available regarding the manufacturing and chemistry of the adhesive. Using the Material Safety Data Sheet (MSDS), marginal constituents of the adhesive have been identified i.e. epoxy resins, synthetic rubber, phenolic polymer and dicyandiamide. Since the dominant constituent is epoxy resin, it will be emphasized in detail.

\section{Epoxy resins}

Epoxy resins offer excellent adhesion to metals and thermoset composites and possess superior properties such as high strength, high stiffness, excellent creep resistance, superior fatigue resistance and high thermal resistance. The epoxy resin structures are characterized by the<smiles>CC(C)(c1ccc(OCC2CO2)cc1)c1ccc(OCC2CO2)cc1</smiles>

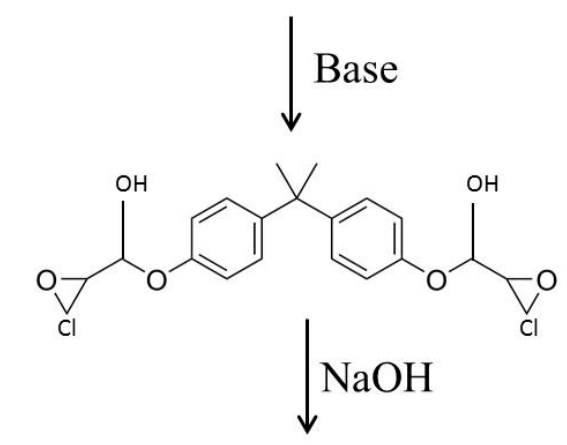

$\mathrm{NaCl}$

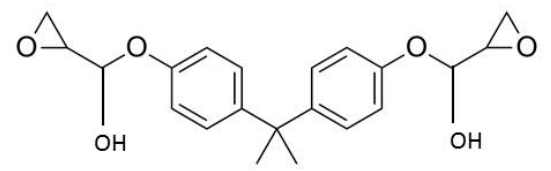

DGEBA

Figure 2.1: Synthesis of di-glycidyl ether of bis-phenol-A with epichlorohydrin. 
presence of the epoxy group i.e. an oxirane ring as their reactive moiety. The epoxy or epoxide group is usually present as a glycidyl ether, glycidyl amine or as part of an aliphatic ring system. The ring structures possess a three membered ring with two carbon atoms individually bonded to an oxygen atom. The crosslinking polymer is the product of the resin mixed with hardener or a curing agent. Epoxy adhesives can be either single component, a heat curing adhesive or multiple component adhesives that can be cured at room temperature or elevated temperatures. One of the most widely used epoxy resins for structural adhesives is produced by the reaction between diglycidyl ether of bisphenol-A (DGEBA) with epichlorohydrin leading to epoxy resin as shown in Figure 2.1.

\section{Phenolic resins}

Combinations of epoxy and phenolic resins provide superior high temperature resistance structural adhesives. These types of resins act as tackifiers or cross linking agents [3] which improvise adhesion. The cross linking ability of phenolic resins is employed to provide strength and durability to an adhesive bond. Phenolic resins can be of three types a) Vinyl phenolic resins, b) Nitrile phenolic resins and c) Epoxy phenolic resins. Of the three resins systems, epoxy resin is a preferred cross linking agent for adhesive bonding of aircraft composites due to its high thermal resistance i.e. $200^{\circ} \mathrm{C}$. On the other hand, vinyl and nitrile phenolic resins can have temperature resistance of $100^{\circ} \mathrm{C}$ and $175^{\circ} \mathrm{C}$ respectively [4].

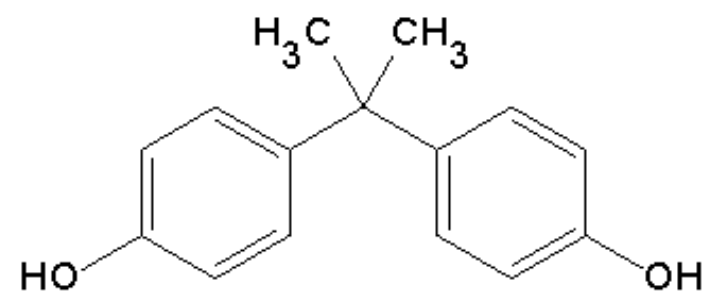

Figure 2.2: Molecular Structure of bis-phenol-A [5]. 
The phenol group of bis-phenol-A is formed when phenol reacts with acetone at a 2 to 1 mole ratio leading to bis-phenol-A of Acetone. If the same reacts with formaldehyde in the same ratio, then it yields bis-phenol-F of Formaldehyde. The molecular formula of bis-phenol- $\mathrm{A}$ is $\mathrm{C}_{15} \mathrm{H}_{16} \mathrm{O}_{2}$ with a molecular weight of $228.291 \mathrm{~g} / \mathrm{mol}$. The structure of bis-phenol-A is shown in

Figure 2.2.

\section{Dicyandiamide (DICY)}

A dimer of cyanamide or also called as cyanoguanidine is a nitrogen based molecule that is highly reactive with epoxy resins and has a molecular formula of $\mathrm{C}_{2} \mathrm{H}_{4} \mathrm{~N}_{4}$. In structural adhesives, it acts as a curing agent to cure DGEBA epoxy resins at elevated temperatures. However, both epoxy resins and DICY are stable at room temperatures. The structure of DICY is shown in Figure 2.3. Exclusive studies on epoxy-DICY reaction have been reported $[6,7,8,9]$ where DICY acts as a hardener in the reaction and plays a key role in transforming heterogeneous systems to increasingly homogenous systems.

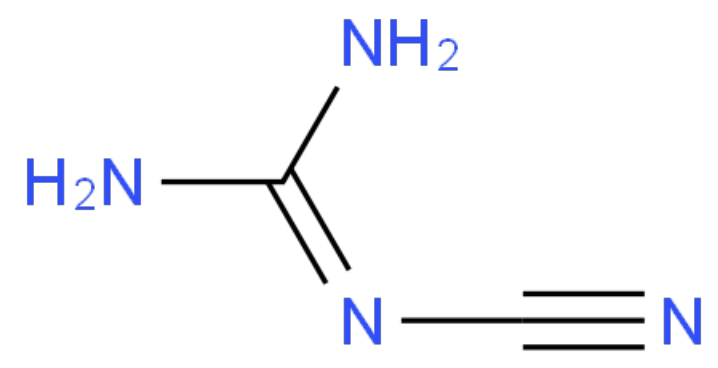

Figure 2.3: Molecular structure of DICY [10]

Some of the resin chemistries described above are used in the curing of epoxy networks which are the critical pathways for understanding adhesion between a structural adhesive and a composite laminate. The ramping rates of temperature and pressure, during the reactions also plays a critical role in the formation of a good bond. A gradual rise in temperature typically yields a favorable adhesive bond while a rapid rise in temperature could stress the bond due to severe thermal loading, 
weakening the crosslinking mechanism. Although the specimen might appear to be cured, this type of cure significantly affects the bond strength.

\subsection{Static Strength Quantification of Adhesive Joints}

Quantification of adhesive joint performance is dependent on the various modes of loading i.e. Mode I, Mode II and Mixed Mode I/II. To date, industry typically relies on Mode I (tensile failure) testing methodologies to obtain fracture toughness of the joint while Mode II and Mixed mode I/II are not fully recognized [12]. Adhesive joints offers advantages over mechanical fastening that eliminates stress concentrations [10] and restrains a joint from fiber cuts that arise due to drilling of mechanical fasteners [11]. Additionally, the increasing number of premature failures of the adhesively bonded structures has led in-depth design and strength evaluations of adhesive joints for numerous joint configurations [13]. Several varieties of joints have been investigated in the past such as scarf joints [14], T-shaped joints [15, 16], butt joints [17] and Lshaped joints due to their simple geometry, while single lap joints [18], double lap joints, stepped lap joints and stepped scarf joints [19] progressed due to the type of applied load. Lap shear (LS) and double cantilever beam (DCB) tests are most commonly used test methods to quantify the initial strength of a bonded joint.

While the static strength for joint assemblies noted above has been quantified extensively, improvements to enhance the bond strength of these bonded joints have been a focus area for many researchers. Sun [20] developed advanced hybrid joint configurations with stepped attachments that provided superior performance over conventional L- shaped joints. While all the tests are largely accomplished on flat surfaces, Chiu et al. [21] investigated on several non-flat surfaces and reported a distinguishing difference in structural property by changing the topology of the interface. Several comprehensive reviews on the strength of joints in fiber reinforced plastics [22], overviews on adhesively bonded joints in composite materials [23]; scientific challenges and opportunities of bonded repair of composite aircraft structures [24] have been well addresses to emphasize the static 
strength quantification of adhesively bonded composite joints. Significant research efforts have been focused on characterizing the initial bond strength of joints assemblies where metal-to-metal bonding have been by and large, the most prominent joint assembly approaches while a fewer studies have been reported on composite bonded assembly [10]. An in-depth understanding of the identification of key processing parameters, important material characteristics are of prime importance that could lead to an avenue of acceptable criteria for bonded assemblies, which is yet to be established.

\subsection{Factors affecting composite bond integrity}

A notable limitation of an adhesive joint is the lack of reliability in providing absolute consistency in bond integrity i.e. a joint free of defects, voids or any potential contamination. A few of the factors that affect composite bond integrity are described in the following sections.

\subsubsection{Surface Preparation}

An adhesive joint can surpass the qualification requirements at the time of testing in terms of bond strength and fracture resistance, but little has been known about the longevity of the bond. In some situations, poor practices and manufacturing processes can actually produce adequate bonds when tested immediately after bonding. However, only high quality manufacturing procedures will produce durable, long lasting adhesive bonds. Having a non-homogenous/nonuniform bond, across the bondline will raise concerns over the quality of the bond and subsequently its durability performance.

Surface preparation of the composite surface is one of the key factors that drive the bond quality $[25,26]$. Proper surface preparation requires precise attention to detail in process selection, validation, specification and performance of adhesives and composite surfaces. Bond strength can be significantly enhanced by surface treating the substrates (metal surfaces/ composite laminates) such as abrasion, use of peel ply [27, 28], grit blasting [29], surface functionalization (for thermoplastics) and anodization in case of metal adherends [30, 31]. 
Peel ply fabrics are often used in the manufacture of composite laminates to protect the surface and improve chemical activity upon removal. Additionally, when the ply is removed, the roughness of the surface is increased [32] which can improve mechanical interlocking of the adhesive with the substrate. However, the peel ply fabric can react with the laminate during fabrication and upon removal, leave residues on the surface [33, 34] which can affect the bond strength of a joint significantly. Additionally, the type of peel ply used has also been known to significantly reduce the bond strength [35] including, nylon or several grades of polyester. With these peel plies, proper surface preparation after the peel ply removal is required in order to eliminate the residues that remain from peel ply.

Techniques such as solvent cleaning for metals, sanding, and gritblasting for composites can enhance the ability of a surface to bond with the adhesive and have been shown to be effective $[36,29]$. However, their reliability and variations in application have yet to be established for certification. Alternatively, atmospheric plasma treatment on metal adherends are known to change the surface chemistry of the metal resulting in an efficient surface favorable for bonding [37, 38]. Laser ablation has shown promise in terms of reliability by creating surfaces free of peel ply residues through a controlled resin removal process from the surface of a composite via a laser beam [39, 40]. This provides superior control and reproducing clean surface topologies.

\subsubsection{Contamination and its effects on durability}

Contamination is termed as a foreign material that interferes with the bonding between an adherend and adhesive. Sources of possible contamination include peel ply residues, release agents, skin oil, dust and moisture [41]. Moisture can arise from the environment or from curing systems that involves steam at elevated temperatures. There have been several studies on moisture absorption in composites [42], particularly relating to pre-bond moisture [43]. Presence of all the aforementioned sources could be treated as contamination and are a global concern in the production of durable adhesive bonds. The contamination from peel ply and their release agents as 
discussed in section 2.2.1 is known to significantly degrade bond quality, which results in reduced adhesion of bonded composite systems [44]. Another type of defects from contamination could be non bonding surfaces that does not allow the adhesive to chemically bond to the adherend due to surface asperities. These are also known as kissing bonds or zero volume bonds. Presence of kissing bonds is in itself an in-homogeneity or a discontinuous bond which could significantly affect bond strength and can deteriorate entire structure over time. Therefore, there is a great need for the assessment of the effects of contamination on bond strength and its durability in ABCJ's. One of the major drawbacks in bonded composites is the detection of the presence of kissing bonds.

Several inline quality control tools such as non-destructive inspection (NDI) [45] and nondestructive testing (NDT) [46] have emerged to validate composite surface and also to detect bond flaws or voids or any possible contamination. Inspection methods such as a manual tap hammer [47], automated tap hammer [48], mechanical impedance analysis [49], C-Scan inspection via ultrasonic testing $[50,51]$ etc., have been developed to detect bond flaws such as a void or foreign material in a macro scale, however, these tools cannot determine if a weak bond (i.e. kissing bond) exists due to the resolution (submicron range) of the resultant acquired information being poor. These weak interface bonds could further deteriorate and lead to debonding at the bonded site over time and compromise the mechanical integrity and structural efficiency during service. With these limitations, NDI cannot reliably ensure the quality of a bond and cannot provide an assessment of the overall strength. Therefore, detecting and testing bond flaws post bonding have been one approach to address the weak bond strength issue. Another approach is to create a weak link such as a foreign material or contamination on the composite surface prior to bonding and investigate its effect on the overall bond strength.

Contamination on surfaces has been significantly reported in the literature as a critical factor that affects adhesion. Recently, Jeenjitkaew et al. [52] contaminated the interface between the metal and adhesive on a double lap joint and observed a $27 \%$ reduction in the bond strength. 
Critchlow et al. [53] also observed a reduction in bond strength with frekote contaminant. Markatos et al. [54] observed a 70\% reduction in fracture toughness after composite panels were dipped into frekote (4 at \% Si) followed by thermal treatments and bonding. Van Voast et al. [55] proposed a methodology to contaminate the peel ply at various levels during its manufacture. In this study, the contaminant was directly applied on the composite laminate, unlike the study conducted in [55], complicating the quantification of the contamination level. Although some research has been conducted on contamination effects, there is a lack of repeatability in the evaluation of the effect of contamination on an adhesive joint. The repeatability and scalability of a contaminants effect on bond strength are of primary importance for understanding the acceptable level of bond degradation without compromising the overall bond performance.

\subsection{Quality Control (QC)}

Obtaining continuous bond homogeneity across the bondline is a primary concern in adhesively bonded joints as mentioned in section 2.3.1. For example, the tests conducted on specimens should be a true representation of the actual material utilized to validate the integrity of the joint. This is non-conventional in adhesive bonding, as there are several other factors that govern the bonding process such as temperature and pressure rates, cooling rates, no foreign material contamination etc. A little variation in the process control could significantly alter the property stability of the specimen. Thus, QC processes should be utiilized in order to ensure the performance of the final product. This enables the progression of a product to meet the performance standards that are set during qualification of the bonded structure. Processes such as accurate layup control, consistent surface preparation methods and precise cure control are a few QC methods industry typically utilizes. Few of outcomes that could result upon improper QC are not utilized are listed below:

- Inadequate pressure applied during lay-up process leads to air channels within the layered laminae that could severely affect the volume fraction of material and introduces porosity. 
- Foreign materials that are trapped in between laminae could affect adhesion of laminate by itself. These could typically arise from the incomplete removal of backing film that a prepreg is covered with.

- Mis-orientation of plies will alter the final product significantly and sometime forgotten plies. Care has to be taken to always record the number of plies that are layered one by one.

- Any potential contamination on the to-be bonded surface acts as a foreign material and could affect adhesion.

- Material cuts and fiber breaks could lead to inconsistent adhesion.

- Controlling the length of the cure time, heat rate, temperature boundaries at the maxima and minima and the cooling rate should be strictly followed.

The degree of detail that needs to be followed for these advanced manufacturing techniques and the associated QC processes will ensure repeatable products with reliable performance. As explained in section 2.3.2 several inline quality control tools have been developed to inspect finished parts for a potential defect or a damaged site. As a result, QC procedures are needed to ensure the continued airworthiness of the bonded structures.

\subsubsection{Bondline control}

Properly designed adhesive joints are never critical in the adhesive layer and rather fail in the adherend i.e. failure shifts towards adherend or the weakest link in the bonded system. To achieve this, the adhesive thickness effects needs to be considered when characterizing the bondline. Several investigations on adhesive thicknesses reported a bondline range between 0.05$0.5 \mathrm{~mm}$ for large joint configurations resulting in superior static strength [56, 57, 58]. Gleich et al. [56] evaluated adhesive stresses for various bondline thicknesses and concluded that they decrease with increasing bondline thickness. Although Goland and Reissner [59] predicted similar behaviour, their prediction was limited to thickness aspects and the stresses at the interface along the bondline was not addressed. However, Lee et al. [60] found an interface damage zone with 
adhesive layer less than $1.5 \mathrm{~mm}$ with metal adherends while the damage was in the near field of precrack with the adhesive layers of $0.1 \mathrm{~mm}$. Additionally, the effect of interfacial crack in adhesive bonded joints with composite substrates was investigated by Yang et al. [61] where the bonded joint was assumed to have no flaws and voids. While, Yang et al. addressed the interfacial crack in a bonded joint assuming no flaws, Goh et al.'s numerical models predicted the damage behaviour of a composite scarf joint at a bondline flaw size of around $3 \mathrm{~mm}$. The data from these articles indicate that bondline thickness is usually controlled or optimized based on the nature of loading, type of the joint and property of adhesive. A need for consistent QC techniques, however, is essential to maximize the bond strength.

\subsubsection{Surface preparation}

Surface preparation on composite laminates require a strict QC methodology that can identify critical process parameters and eliminate improper practices such as

- Inappropriate sequence of surface preparation approaches followed during sanding or grit blasting.

- Non-maintenance of manufacturers recommended ambience while surface preparations.

- Improper solution concentrations during cleaning.

- Not changing abrasives periodically if sanding or grit blasting.

- Elongated periods of exposure after surface preparation.

All the above mentioned QC guidelines are documented based on experience at the field stations and could lead to non-homogeneous surfaces which adversely affects the bonded system.

\section{$2.5 \quad$ Test Methods}

Designers of composite bonded structures need significantly more information and material property measurements in comparison to working with metals such as independent measurements of tension, compression and in-plane shear properties. Metal bonds or Composite bonds have their distinctive failure modes relating to their respective loading conditions. 
Additionally, the mechanical properties of composite materials and laminates tend to have higher variability than their metallic counterparts. The advisory circular issued jointly by Federal Aviation Administration (FAA) and the European Aviation Safety Agency (EASA) details out the design, manufacturing and maintenance acceptable certification requirements for composite aircraft structures which is one of the most cited articles in relation to light weight structures for aviation safety [62]. Toughness of the adhesive is universally determined as a measure of a materials ability to absorb work done on it. Also defined as the actual work per unit volume or unit mass of material that is required to rupture it.

As mentioned in section 2.2, there are numerous experimental studies that have been conducted to evaluate bond strength and there is little agreement on the best practices for the durability assessment of bonded joints. This subject is probably the major challenge that the adhesion community faces today. The following test methods described provides an overview on the research conducted on the durability of adhesive bonds. Some initial static strength evaluations are also mentioned which serve as the building blocks in the design and development of advanced structures for aircrafts and for their universal acceptance and certification.

\subsubsection{Wedge Crack Tests}

One of the most popular methods to evaluate the durability of a bond is the wedge crack test [64]. In these tests, adhesively bonded metal specimens are loaded by forcing a wedge into one end of the laminate resulting in a tensile stress in the region near the crack tip (Mode I) as shown in Figure 2.4. This represents a typical wedge crack test. The initial crack arrests when the tensile stresses are just below tensile ultimate for the adhesive. That leaves the interface under extreme stresses and any degradation of the interface at this point, such as hydration, could result in interfacial failure [63]. Results from the wedge test provide measurements of crack growth rate and mode of failure (cohesion, adhesion, or adherent failure). It is typically used to evaluate durability by subjecting the wedged specimens to an accelerated aging environment. A significant 
advantage is the fact that multiple specimens can be easily fabricated and subsequently subjected to a variety of environmental conditions. Another advantage stems from the concept that the most important aspect of durability testing is the mode of failure. With the wedge test, the force to drive in the wedge is neither measured nor recorded [64]. The disadvantage being, the varying loading condition that is created as the crack propagates in the bondline. Because of its potential to evaluate durability of adhesive bonds, previous studies have adapted the wedge test to evaluate bond strength durability in composite joints. To prevent diversion of the delamination, unidirectional $\left(0^{\circ}\right)$ tape plies oriented in the longitudinal direction of the specimen was proposed by Hart-Smith [64].

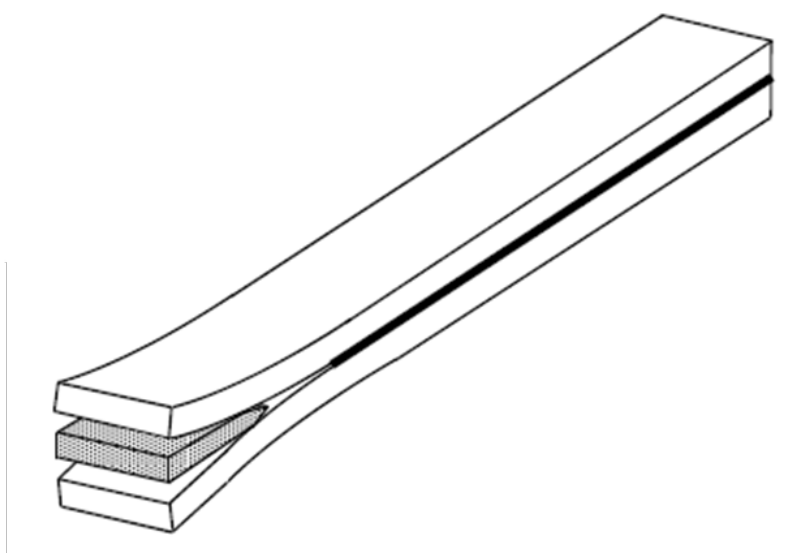

Figure 2.4: Schematic showing Wedge Crack Extension Test [65]

Numerous studies have been conducted using the wedge test as the basis for durability evaluation. Some of those include Adams et al. [66], who used the wedge test with metal adherend specimens in high temperature water to evaluate various durability test methods including DCB tests and variations of the wedge test (forced wedge test). Bardis and Kedward [67] conducted static wedge tests with partially wedged composite samples in water, sulfuric acid/water solution and sodium hydroxide solution. Overall wedge test method is a well suited test for assessing adhesive 
bond durability however, wedge insertion rates and their effects on initial crack length need to be well established.

\subsubsection{Shear Tests}

Lap Shear tests are one of the most widely utilized shear testing methods for assessing initial adhesive bonds strength loaded in shear. However, due to the ease of manufacturing, lap shear test specimens have been extended to evaluate durability as well. This test consists of two substrates bonded together to create a specimen that is tested under tension loading as shown in Figure 2.5. For a single lap, the tension force results in a shear (Mode II) between the substrates and the adhesive. Details of typical testing procedures and dimension requirements are provided in ASTM D1002.

The use of lap shear tests for evaluating bond quality has generated controversy among authors. Davis and Tomblin [14] conducted a survey of 20 organizations that indicated 77 percent of designers use lap-shear test results to establish design allowables. However, the authors indicate that its use is not considered best practice. Furthermore, Bardis and Kedward [67] found that "lap shear tests provided limited verification of bonded assembly reliability, especially when considering prolonged loading and environmental conditions”. They also note that a pure shear loading condition is rarely achieved in practice. Regardless of the disadvantages, the lap shear test remains a popular test approach due to its simplicity and ease of implementation. The following review covers a few of the lap shear and slight variations of the lap shear test. The effect of test temperature and prebond moisture on carbon fiber reinforced polyester composites was investigated by Parker $[68,69,70]$ using single lap joints. Cyclic environmental ageing was studied by $\mathrm{Xu}$ et al. [71], using metal to composite adherends in lap shear tests. Other researchers have extended the preconditioning to include mechanical loading in addition to exposure to harsh environments. Briskham and Smith [72] evaluated both metal to metal adherends and metal to composite adherends using lap joints with cyclic mechanical loads in a hot water environment. 
Smith and Pothakamuri [73] studied the effects of creep loading in a hot wet environment using various peel ply and moisture conditions. It should be noted that their tests were conducted using thick wide lap shear coupons. Knox and Cowling [74] used a thick metal adherend that was conditioned with environmental aging in a lap shear test. Their efforts focused on understanding the effects of various surface preparation techniques. Other variations of the lap shear tests include work by Ashcroft et al. [75], who used double lap joints with composite adherends to evaluate the effect of test temperatures. They also studied durability by combining the effect of environment aging and fatigue on lap-strap joint specimens.

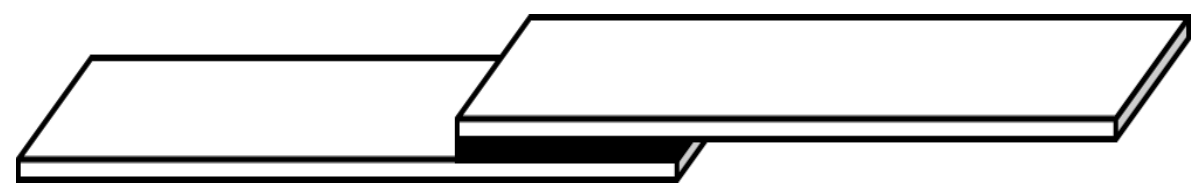

Figure 2.5: Schematic Showing Lap Shear test coupon

\subsubsection{Interlaminar shear strength (ILSS) Tests}

Interlaminar shear strength type of tests provide apparent shear strength of the material where the stress distribution is assumed to be isotropic. Chaterjee et al. [76] evaluated several test methods to determine shear properties of composite materials consisting of high modulus, high strength fibers in organic matrix materials where an extensive source of information can be evaluated and test methods reliable to application can be chosen from. Yokoyama et al. [77] evaluated the ILSS and In-Plane Shear Strengths (IPSS) of unidirectional carbon/epoxy lamianted composites using a standard Hopkinson pressure bar and found that ILSS is lower than IPSS by $29 \%$ at low and high rates of deformation. No failures were evidenced and only shear strengths were considered. Vodicka et al. [78] investigated the effects of moisture uptake, ultraviolet (UV) degradation, temperature on the strength of graphite/epoxy composites for 468-520 weeks and 
found a marginal increase in the ILSS with absorbed moisture and attributed it to the combined effects of stress relief and plasticization of the matrix.

\subsubsection{Double Cantilever Beam Test}

A number of studies have been performed in which DCB tests were adapted for adhesively bonded composites. The double cantilever beam (DCB) test is a convenient testing method for the study of crack propagations and arrest, and is a standardized experimental method that was established to determine the interlaminar fracture toughness and delamination growth onset resulting from a Mode I loading condition (ASTM D5528, D6115, D3433) as shown in Figure 2.6. This test provides data for the energy release rate, crack growth length, and also provides the dominant mode of failure. To determine the effects of durability, on bonded systems, the specimens are often conditioned via environmental exposure prior to testing. A few of these studies that address durability are briefly described in this section.

Although lap-shear type tests are the most popular test for assessing adhesive bond strength, Bardis and Kedward [67] reported that mode I tests are best for durability. This is due to the inability of lap-shear tests of metal substrates to duplicate service disbond failures over a range of temperatures and environments. Hart-Smith noted that a limitation to DCB testing is that only one specimen can be tested at a time, yielding inefficient testing [64].

DCB tests can also be used to evaluate a number of adhesive bonding process parameters including surface preparation methods, precure moisture in peel ply [33] and contamination effects [79]. While, the above mentioned parameters influence the initial bond strength of a bonded joint, a number of researchers have used DCB testing on specimens preconditioned with environmental aging of adhesive bonds. Adams et al. [66], Xu et al. [71], and Datla et al. [80], all studied the effects of temperature and humidity of metal or conductive laminates on strain energy release rates obtained using DCB tests or slight variations of DCB testing. Lee [81] evaluated composite laminates in cryogenic temperatures using DCB testing. Other researchers have studied the 
combined effects of fatigue loading and environmental aging. Johnson et al. [82], used DCB specimens to evaluate bond durability for composite/metal joints by exposing specimens to hot/wet environments prior to fatigue testing. Kinloch et al. [83], used aluminum substrates in tapered DCB specimens to evaluate simultaneous mechanically loading and environmental aging. Smith and Pothakamuri [73] created a frame that allowed for similar testing conditions and evaluated the durability of adhesively bonded composite substrates.

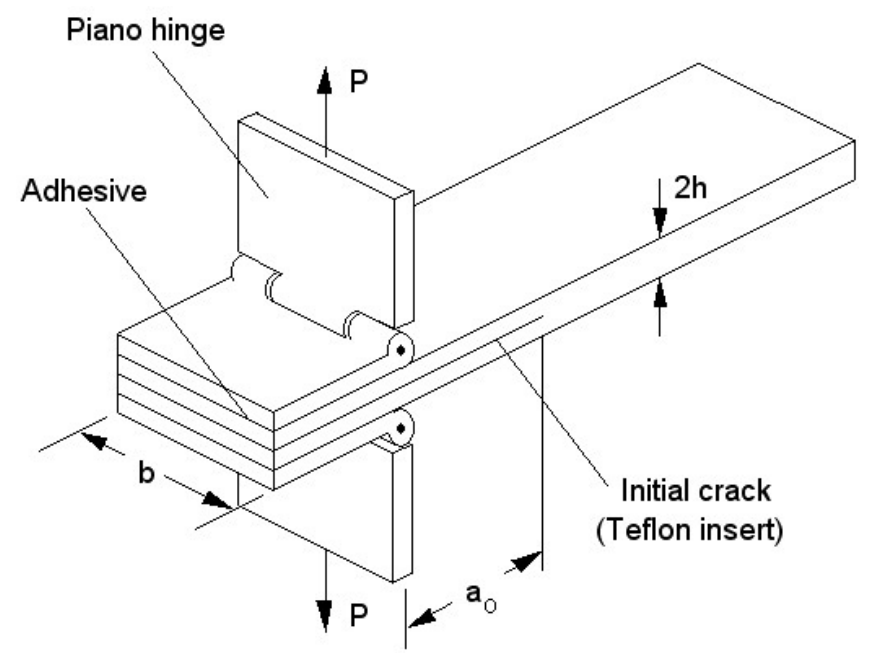

Figure 2.6: Schematic of a Double Cantilever Beam test specimen

\subsubsection{Overview of Test Methods}

The fracture mechanics test methodologies described above are determined based on their modes of loading and their test specimen geometry. The approaches mentioned determine the propagation characteristics of existing cracks/ delamination's within a bonded assembly and how durability affects the bonded joint based on the joint configuration. A common practice in testing ABCJ's is typically loading the specimens in Mode I, Mode II and Mixed mode I/II but due to their simple geometry and uniformity, DCB testing is recognized and the preferred testing methodology for bonded joints in adhesion community. The main advantage of a DCB test is its wide utilization of test specimen i.e. it permits measurements in Mode I, Mode II or mixed mode. Mode II and Mode I/II can typically possess identical geometry but the fracture and its mode of failure depends on the remotely applied loads. The durability of the composite can be at compromise with the 
conditions mentioned and hence the durability studies on the composites and especially bonded composites have to be well understood prior to their qualification or being well recognized for their usage in the aircraft structures.

\subsection{Surface Characterization}

In aerospace community, adherends and adhesives for composite materials fall under the category of epoxy resins. Characterization of these polymeric compounds is critical in understanding the chemistry associated with the surfaces. This will be able to qualify the characterization technique utilized as a QC method for producing reliable and durable surfaces. A major challenge with the use of adhesively bonded composites is the lack of understanding of the surface chemistry of substrates prior to bonding and its effect on bond strength. Since adherends and adhesives involved in adhesively bonded composite systems undergo various surface preparation methods, surface characterization studies will aid in understanding and quantifying differences in the preparation methods adopted. This characterization becomes extremely complex for in-situ monitoring processes of epoxy resins such as curing and ageing processes where the changes are monitored as a function of cure/time.

\subsubsection{Surface Analytical Tools}

\subsubsection{FTIR}

An FTIR uses a transmission sampling technique that involves passing the infrared energy through the sample and detecting that portion of the beam that is transmitted, i.e. not absorbed. The infrared beam passes through the sample and the energy that comes through the sample is measured versus the respective wavelength to generate a spectrum. Numerous studies on infrared characterized polymeric systems exist in the analytical community that can clearly distinguish between the chemical composition and the microscopic constituent structure of a polymer composite [84, 85, 86]. Chen et al. [87] studied the fiber matrix interface of carbon-fiber epoxy composite and concluded that the size of carbon fiber is important in governing the performance of 
composite as the fiber and matrix result in the formation of a double interface which improves adhesion. In relation to surface preparation approaches Tracey and Flinn [88] compared current state of the art handheld FTIR instruments from two different sources and understood the effects of surface preparation on IR spectra. They reported potential applications of FTIR as a QA technique in adhesive bonding process and how FTIR can quantify the changes associated with surface preparation. Fata et al. [89] investigated on the aging behavior of epoxy-DICY network and characterized critical chemical and structural changes in the epoxy-DICY network during aging. Flinn et al. [90] mapped composite surfaces (ply by ply) via FTIR to detect thermal damages and tried to correlate the mapped surfaces to their mechanical strength. However, no clear correlation was obtained at that point in time.

\subsubsection{Contact Angle}

To understand the wetting characteristics of the composite surface prior to adhesive bonding, their surface energy needs to be evaluated in order to determine the wettability of the adhesive on adherend. This is one of the critical surface properties that govern the adhesion phenomenon of an adhesive apart from its bulk properties [91]. A significant change in the wetting behavior of the composite was reported by Hameed et al. [92] where poly(styrene-co-acrylonitrile) was blended with epoxy resin system. Hydrophobic nature was observed in the blended composite with an increase in contact angle when compared to pristine resin with no blend. This suggests that contact angle could detect changes associated with the surface with and without foreign materials that aid in quantifying the changes. Although water interactions with polymer surfaces were studied earlier [93], very little has been researched on the effect of surface types on bond performance in ABCJ's. Dillingham et al. investigated on abraded and non-abraded composite surfaces and correlated their surface free energy with the bond strength [94]. In another study, Dillingham et al. [95] was able to distinguish the detrimental levels to acceptable levels of siloxane contamination 
(residue of peel ply) via wettability measurements prior to affecting the bond strength. This also serves as a potential QA method on qualifying the preparedness of a surface prior to bonding.

\subsection{Moisture Uptake}

The amount of moisture in a material determined under prescribed condition and expressed as a percentage of the mass of the moist uptake in a specimen is termed as moisture content. Moisture uptake criteria in epoxy systems have been widely investigated [96, 97, 98], however, their water absorption characteristics have not been very well understood. Their response can be quite unexpected depending on the environment i.e. if the epoxy systems are subjected to critical temperatures or unusual pressure history [99]. Several researches generated material property data for varying levels of saturation in order to quantify the effects of moisture ingression on mechanical properties [100, 101]. Diffusion of moisture is the only route for moisture to be absorbed in a composite matrix or a fiber following Fick’s law. Parker B.M. [100] investigated single lap joints that were cured at $120^{\circ} \mathrm{C}$ and $175^{\circ} \mathrm{C}$ and environmentally conditioned them for an extensive period of 1000 , 4000 and 9000 hours of exposure to over saturated $\mathrm{K}_{2} \mathrm{SO}_{4}$ solution at $50^{\circ} \mathrm{C}$ and $96 \% \mathrm{RH}$. Joints cured at $120^{\circ} \mathrm{C}$ were affected more than joints cured at $175^{\circ} \mathrm{C}$, showing that moisture affects on bond strength is dependent on cure temperature. Obrien [104], suggested that the integrity of a bond can be preserved, if the interface is strong and reported that presence of moisture at the interface could deteriorate the bond significantly. It was also attributed that at the localized site of the strongest interface, the rate limiting step for failure is more pronounced with the presence of moisture and the role of interfacial diffusion becomes the least important factor. Efforts were made by Nguyen et al. [104], to measure the water concentration at an epoxy/SiO ${ }_{2}$ interface using infrared spectroscopy. The weak adhesion obtained was attributed to the accumulation of water which was well correlated. These studies showcase the diffusion of water at the interface and its deleterious effects on the integrity of the bond. An in-depth study by Bowden and Throssell [106] 
on diffusion of water inside metal adherends such as aluminum, iron and $\mathrm{SiO}_{2}$ surfaces revealed 20 molecular layers thick of water layer at ambient temperatures and humidity.

While most of the literature indicated the degradation of mechanical properties of an epoxy composite systems upon moisture ingression, there is a contradictory agreement revealing an enhancement in mechanical properties with increasing moisture to a certain level followed by its degradation. Selzer and Friedrich [101] investigated the moisture absorption properties on epoxy systems by immersing specimens in water at $23^{\circ} \mathrm{C}, 70^{\circ} \mathrm{C}$ and $100^{\circ} \mathrm{C}$ for different crack opening modes and observed an increase in $G_{1 C}$ values for fully saturated specimens when compared to the dry specimens.

In a bonded system, the material surface that is in direct contact with the moist environment absorbs moisture rapidly, while moisture transport to the inner layers of a composite is relatively slow. The diffusion rate is dependent on the thickness of the system and is of many orders of magnitude slower.

Some composite systems exhibit matrix contraction when exposed to elevated temperatures [104], introducing stress concentrations around the fibers. An similar effect is observed in composite materials upon physical aging. This research investigates typical materials used in aircraft grade bonded composite joints that are subjected to long term exposure. This will aid in providing information about the saturation limit of the bonded system and the time taken for it to reach moisture equilibrium. Thereby, determining the effects of ageing, time dependent phenomenon that is related to durability on the overall bond performance. This will aid in assessing the changes in mechanical behavior as a consequence of ageing.

\subsection{Durability}

The current testing and environmentally conditioning of adhesive bonds is not fully understood with little to no agreement of common practices in the bonding community. Silva [95] notes, "Durability is a general term that is related to the residual strength of the joint when subjected 
to water or temperature”. Environmental conditioning is also referred to as aging where the materials to be tested are exposed to specific environments for a definitive period of time. This is often conducted in conjunction with elevated temperatures and relative humidity. Issues associated with the durability and related failures in aircrafts, continue to be a critical hindrance for the universal acceptance of ABCJs $[109,110]$. The assessment of bond strength and durability (long term performance) of aircraft structures is affected by extrinsic factors such as environmental conditions [71], operating temperatures [68, 69], relative humidity [111], service loads [112] and cleanliness of bonding surface $[67,64]$. Several in-house test procedures have been developed and put forward to work in accordance with own custom set standards. This may be due to a lack of knowledge on test methodologies or appropriate standards that needs to be adopted or due to the failure in mimicking the durability standard that represents actual service conditions. To date, methods to evaluate durability have focused on the environmental conditioning of specimens in an unstressed condition. However, a bonded structure in service experiences stress as well as environmental conditioning at the same time. Failure to account for the combined effect of stress and environment will frequently result in an overestimation in strength and fatigue life [114]. Thus, standard test methods need to incorporate appropriate conditions and be capable of represent actual service conditions.

\subsection{Summary}

This literature review covers a wide spectrum of aspects related to adhesively bonded composite joints including; a) structural adhesives used in adhesive bonding and the chemistry associated with it; b) factors that affect composite bond integrity such as surface preparation and contamination; c) use of surface analytical tools in characterization of ABCJ's; d) quality control methods and e) issues related to durability and their effects on ABCJ's. These are the important 
factors that the bonding community must understand and in particular, understanding how contamination influences bond strength and durability. The utilization of materials, properties and chemistry in relation to the adhesion theories enlightens the mechanism of bonding processes that needs to be well understood. This knowledge will serve as the foundation for the level of quality control that needs to be adopted in bonding of composite joints. The pros and cons of surface analytical tools such as FTIR and Contact angle measurements will assist researchers in utilizing suitable techniques that determines the preparedness of the surfaces that are to be bonded. Finally, without the knowledge of durability of ABCJ's, there is no statistical basis for the reliability of bonded joints especially in primary structures and hence intelligent design methodologies needs to be adopted to gain confidence in the reliable and repeatable performance of bonded joints, thus providing a platform for their certification and qualification. This summary is an effort to provide a necessary background information needed for the study of adhesively bonded composite joints, the work that has been accomplished in this area and the critical issues associated with bonded joints. 


\subsection{References}

[1] H. Parvatareddy and D. Dillard, "Effect of mode-mixity on the fracture toughness of Ti6Al-4V /FM-5 adhesive joints," International Journal of Fracture, vol. 96, no. 3, pp. 215228, 1999.

[2] A. Kinloch, Adhesion and Adhesives: Science and Technology, London: Chapman and Hall, 1987.

[3] G. B. Schneberger, Adhesives in Manufacturing (Manufacturing Engineering and Materials Processing) 1st Edition, CRC Press, August 1983.

[4] Y. a. Tancrede, "Phenolic Adhesives and Modifiers," in Adhesives in Manufacturing (Manufacturing Engineering and Materials Processing) 1st Edition, CRC Press, August 1983, pp. 237-268.

[5] P. W. Vittum and G. H. Brown, "Indoaniline Dyes. III. Coupling of para-Substituted Phenols with Oxidized p-Aminodimethylaniline," Journal of American Chemical Society, vol. 71, no. 7, pp. 2287-2290, 1949.

[6] U. Muller, M. Philipp, J. C. Gaukler, W. Possart, R. Sanctuary and J. K. Kruger, "Dissolution, Transport and Reaction at a DICY/DGEBA Interface," The Journal of Adhesion, vol. 88, no. 3, pp. 253-276, 2012.

[7] J. C. Gaukler, "Oligomer Formation in Epoxy-Dicyandiamide Systems," The Journal of Adhesion, vol. 88, no. 8, pp. 720-750, Feb 2012.

[8] S. R. Mousavi and I. A. Amraei, "Toughening of dicyandiamide-cured DGEBA-based epoxy resin using MBS core-shell rubber particles," Journal of Composite Materials, vol. 0, no. 0, pp. 1-7, 2014.

[9] M. D. Gilbert, Mechanism and kinetics of the dicyandiamide cure of epoxy resins, Amherst: PhD Dissertation, University of Massachusetts-Amherst, 1988.

[10] A. Kinloch, B. Blackman and W. Teo, "The Adhesive Bonding of Polymeric Matrix Composites," in 17th International Conference on Composite Materials (ICCM), Edinburgh, UK, July 2009.

[11] A. Parashar and P. Mertiny, "Adhesively bonded composite tubular joints: Review," International Journal of Adhesion and Adhesives, pp. 58-68, 2012. 
[12] A. Kinloch, "Industrial applications of Adhesive fracture mechanics," The Adhesion Society 2008, Austin, TX, 2008.

[13] S. Pantelakis and K. Tserpes, "Adhesive bonding of composite aircraft structures: Challenges and recent developments," Science China: Physics, Mechanics \& Astronomy , vol. 57, no. 1, pp. 2-11, January 2014.

[14] A. Kimiaeifar, H. Toft, E. Lund, O. Thomsen and J. Sorensen, "Reliability analysis of adhesive bonded scarf joints," Engineering Structures, vol. 35, pp. 281-287, February 2012.

[15] A. Abdullah, M. Afendi and M. Abdul Majid, "Effect of Adhesive Thickness on Adhesively Bonded T-Joint," in International Conference on Mechanical Engineering Research (ICMER), Pahang, Malaysia, July 2013.

[16] W. Broughton, L. Crocker, M. Gower and R. Shaw, "Assessment of Predictive Analysis for Bonded and Bolted T-Joints," National Physical Laboratory (NPL), West Yorkshire, UK, May 2004.

[17] L. Crocker and G. Dean, "Tensile Testing of Adhesive Butt Joint Specimens," Crown Copyright, Middlesex, UK, May 2001.

[18] A. Chadegani and R. C. Batra, "Analysis of adhesive-bonded single-lap joint with an interfacial crack and a void," International Journal of Adhesion and Adhesives, vol. 31, no. 6, pp. 455-465, September 2011.

[19] L. Hart-Smith, "Adhesive Bonded Double Lap Joint," National Aeronautics and Space Administration (NASA), Hampton, Virginia, Jan 1973.

[20] C. Sun, "Adhesively Bonded Joints, Part I: Bondline Thickness Effects and Hybrid Design of Adhesively Bonded Joints," U.S. Department of Transportation, Federal Aviation Administration (FAA), Renton, WA, Feb 2014.

[21] S.-H. Chiu, Adhesively bonded joints with non-flat interfaces, Mechanical Engineering Master's Thesis, Northeastern University, Jan 2011.

[22] F. Mathews, P. Kilty and E. Godwin, "A reveiw of strength of joints in fiber-reinforced plastics 2: adhesively bonded joints," Composites, vol. 13, no. 1, pp. 29-37, 1982.

[23] M. Banea and L. da Silva, "Adhesively bonded joints in composite materials: An overview," Jounrla of Materials Design and Applications, vol. 223, no. 1, pp. 1-18, 2009. 
[24] K. Katnam, L. da Silva and T. Young, "Bonded repair of composite aircraft structures: A review of scientific challenges and opportunities," Pregress in Aerospace Sciences, vol. 61, pp. 26-42, 2013.

[25] L. da Silva, N. Ferreira, V. Richter-Trummer and E. Marques, "Effect of grooves on the strength of adhesively bonded joints," International Journal of Adhesives and Adhesion, vol. 30, no. 7, pp. 35-43, 2010.

[26] L. da Silva, R. Carbas, G. Critchlow, M. Figueiredo and K. Brown, "Effect of material, geometry, surface treatment and environment on the shear strength of single lap joints," International Journal of Adhesion and Adhesives, vol. 29, no. 6, pp. 621-632, September 2009.

[27] B. D. Flinn, B. K. Clark, J. Satterwhite and P. Van Voast, "Influence of Peel Ply Type on Adhesive Bonding of Composites," in Society for Advancement of Materials and Process Engineering (SAMPE), Baltimore, MD, June 3-7, 2008.

[28] J. M. Gardner, J. P. Wolbert, L. R. Holmes Jr. and D. D. Pappas, "Evlauation of Peel Ply Surface Preparation of Composite Surfaces for Secondary Bonding," in SAMPE Conference Proceedings, Baltimore, MD, 2012.

[29] A. Harris and A. Beevers, "The effects of grit-blasting on surface properties for adhesion," International Journal of Adhesion and Adhesives, vol. 19, no. 6, pp. 445-452, Dec 1999.

[30] A. Rudawska, "Selected aspects of the effect of mechanical treatment on surface roughness and adhesive joint strength of steel sheets," International Journal of Adhesion and Adhesives, vol. 50, pp. 235-243, 2014.

[31] P. Molitor, V. Barron and T. Young, "Surface treatment of titanium for adhesive bonding to polymer composites: a review," International Journal of Adhesion and Adhesives, vol. 21, no. 2, pp. 129-136, 2001.

[32] K. Meine, K. Klob, T. Scheider and D. Spaltmann, "The influence of surface roughness on the adhesion force," Surface and Interface Analysis, vol. 36, pp. 694-697, 2004.

[33] M. Phariss and B. Flinn, "The Effect of Peel-Ply Surface Preparation Variables on Bond Quality," U.S. DOT, Federal Aviation Administration (FAA), Washington, 2006.

[34] L. Hart-Smith, G. Redmond and M. Davis, "The Curse of Nylon Peel Ply," in 41st SAMPE International Symposium and Exhibition, 1996.

[35] B. Flinn, B. Cark, J. Satterwhite and P. Van Voast, "Influence of Peel Ply Type on Adhesive Bonding of Composites," in SAMPE 2007, Baltimore, MD, 2007. 
[36] D. J. J. Varacalle, G. P. Donna, D. M. Douglas, W. Rhodaberger and E. Sampson, "Effect of Grit Blasting on Substrate Roughness and Coating Adhesion," Journal of Thermal Spray Technology, vol. 15, no. 3, pp. 348-355, 2006.

[37] A. C. Tracey, M. A. Belcher, K. Y. Blohowiak and B. D. Flinn, "Improving Adhesive Bonding through Surface Characterizations: Reverse the Curse of Nylon Peel Ply?," in Joint Advanced Materials and Structures- AMTAS meeting, Seattle, WA, 2014.

[38] D. Harris and D. Pappas, "Confocal Microscopy Studies for Plasma Surface Modified Films and Fibers," U.S. Army Research Laboratory, Aberdeen Proving Ground, MD, Dec 2006.

[39] F. L. Palmieri, M. A. Belcher, C. J. Wohl, K. Y. Blohowiak and J. W. Connell, "Supersonic Retropulsion Surface Preparation of Carbon Fiber Reinforced Epoxy Composites for Adhesive Bonding," NASA, 2009.

[40] M. A. Belcher, C. J. Wohl, J. W. Hopkins and J. W. Connell, "Laser surface preparation for bonding of aerospace composites," Proceedings of the ICE - Engineering and Computational Mechanics, vol. 164, no. 3, pp. 133-138, Sep 2011.

[41] M. Davis, J. Tomblin and ., "Best Practice in Adhesive bOnded Structures and Repairs," U.S. DOT, Federal Aviaiton Administration (FAA), Seattlte WA., April 2007.

[42] L. Asp, "The Effects of Moisture and Temperature on the Interlaminar Toughness of a Carbon/Epoxy Composite," Composites Science and Technology, vol. 58, pp. 967-977, 1998.

[43] B. Parker, "The effect of composite prebond moisture on adhesive-bonded CFRP-CFRP joints," Composites, vol. 14, no. 3, pp. 226-232, July 1983.

[44] D. Klapprott, H. Li, R. Wong and G. Geisendorfer, "Key Factors of the Peel Ply Surface Preparation Process," Henkel Corporation, Long Beach, CA, 2004.

[45] J. S. Tomblin, K. Raju, J. Acosta, B. Smith and N. Romine, "Impact Damage Characterization and Damage Tolerance of Composite Sandwich Airframe StructuresPhase II," National Technical Information Service (NTIS), Springfield, October 2002.

[46] R. Adams and B. Drinkwater, "Nondestructive Testing of Adhesive-Bonded Joints," NDT \& E International, vol. 30, no. 2, pp. 93-98, 1997.

[47] J. S. Tomblin, L. Salah, J. M. Welch and M. D. Borgman, "Bonded Repair of Aircraft Composite Sandwich Structures," National Technical Information Service (NTIS), Springfield, 2004. 
[48] G. E. Georgeson, L. Scott and H. Jeff, "Electronic Tap Hammer for Composite Damage Assessment," in Proceedings of the Society of Photo-Optical Instrumentation Engineers (SPIE), November 1996.

[49] D. Roach, K. Rackow and R. Duvall, "Innovative Use of Adhesive Interface Characteristics to Non-Destructively Quantify the Strength of Bonded Joints," in 10th European Conference on Non-Destructive Testing, Moscow, 7-11 June 2010.

[50] M. P. Souza, J. M. Rebello and S. D. Soares, "Ultrasonic Inspection of Adhesive Joints on Fiberglass Reinforced Epoxy Pipes," in 16th International Conference on Composite Structures (ICCS 16), Porto, 2011.

[51] M. Wood, P. Charlton and D. Yan, "Ultrasonic Evaluation of Artificial Kissing Bonds in CFRP Composites," The e-Journal of Nondestructive Testing, vol. 19, no. 12, Dec 2014.

[52] C. Jeenjitkaew, Z. Luklinska and F. Guild, "Morphology and surface chemistry of kssing bonds in adhesive joints produced by surface contamination," International Journal of Adhesion and Adhesives, vol. 30, pp. 643-653, June 2010.

[53] G. Critchlow, R. Litchfield, I. Sutherland, D. Grandy and S. Wilson, "A review and comparative study of release coatings for optimised abhesion in resin transfer moulding applications," International Journal of Adhesion \& Adhesives, vol. 26, pp. 577-599, 2006.

[54] D. Markatos, K. Tserpes, E. Rau, S. Markus, B. Ehrhart and S. Pantelakis, "The effects of manufacturing-induced and in-service related bonding quality reduction on the mode-I fracture toughness of composite bonded joints for aeronautical use," Composite: Part B, vol. 45, pp. 556-564, 2013.

[55] P. Van Voast, P. Shelley, P. Blakley, A. Tracey, B. Flinn, G. Dillingham and B. Oakley, "Effect of Varying Levels of Peel Ply Contamination on Adhesion Threshold," in Society for the Advancement of Material Process and Engineering (SAMPE), Seattle, WA, 2010.

[56] D. Gleich, M. Van Tooren and A. Beukers, "Analysis and evaluation of bondline thickness effects on failure load in adhesively bonded structures," Journal of Adhesion Science and Technology, vol. 15, no. 9, pp. 1091-1101, 2001.

[57] H. Adin, "The investigation of effect of adherend thickness on scarf lap joints," Materialwissenschaft und Werkstofftechnik, vol. 44, no. 10, pp. 839-846, 2013.

[58] A. Objois , Y. Gilibert and B. Fargette, "Theoretical and Experimental Analysis of the Scarf Joint Bonded Structure: Influence of the Adhesive Thickness on the Micro-mechanical Behavior," The Journal of Adhesion , vol. 70, no. 1-2, pp. 13-32, 1999. 
[59] M. Goland and E. Reissner, "The Stresses in Cemented Joints," Journal of Applied Mechanics, vol. 11, pp. A17-A27, 1944.

[60] D. B. Lee, T. Ikeda, N. Miyazaki and N. S. Choi, "Effect of Bond Thickness on the Fracture Toughness of Adhesive Joints," Journal of Engineering Materials and Technology, vol. 126, no. 1, pp. 14-18, Jan 2004.

[61] C. Yang, A. Chadegani and J. Tomblin, "Strain energy release rate determination of prescribed cracks in adhesibely bonded single lap composite joints with thick bondlines," Composites Part B: Engineering, vol. 39, pp. 863-873, 2008.

[62] Advisory Circular AC20-107B, "Composite Aircraft Structure," U.S. Department of Transportation Federal Aviation Administration, 2009.

[63] M. Davis and A. McGregor, "Assessing Adhesive Bond Failure: Mixed Mode Bond Failures Explained," in ISASI Australian Safety Seminar, Canberra, June 2010.

[64] L. Hart-Smith, "A Peel-Type Durability Test Coupon to Asses Interfaces in Bonded, CoBonded, and Co-Cured Composite Structures," International Journal of Adhesion and Adhesives, vol. 19, pp. 181-191, 1999.

[65] D. Adams, L. DeVries and C. Child, "Durability of Adhesively Bonded Joints: Revising the Wedge Crack Durability Test," in AMTAS Autumn 2012 Meeting, Seattle, WA, Oct 2012.

[66] R. Adams, J. Cowap, G. Farquharson, G. Margary and D. Vaughn, "The relative merits of the Boeing wedge test and the double cantilever beam test for assessing the durability of adhesively bonded joints, with particular reference to the use of fracture mechanics," International Journal of Adhesion and Adhesives, vol. 29, pp. 609-620, 2009.

[67] J. Bardis and K. Kedward, "Effects of Surface Preparation on the Long-Term Durability of Adhesively Bonded Composite Joints," U.S. Department of Transportation FAA. Report No: DOT/FAA/AR-03/53, Washington, WA, 2004.

[68] B. Parker, "The effect of extended hot-humid exposure of adhesive bonded carbon-fibre composite joints, Part 1: 175 C cured Matrix," RAE Technical Report 89001, Jan 1989.

[69] B. Parker, "The effect of extended hot-humid exposure of adhesive bonded carbon-fibre composite joints, Part II: 120 C cured Matrix," RAE Technical Report 89061, Jan 1990.

[70] B. Parker, "The effect of composite pre-bond moisture on adhesive-bonded CFRP-CFRP joints," Composites, vol. 14, no. 3, pp. 226-232, 1983. 
[71] S. Xu, D. Dillard and G. Dillard, "Environmental aging effects on the durability of electrically conductive adhesive joints," International Journal of Adhesion and Adhesives, vol. 23, pp. 235-250, 2003.

[72] P. Briskham and G. Smith, "Cyclic stress durability of lap shear joints exposed to hot-wet conditions," International Journal of Adhesion and Adhesives, vol. 20, pp. 33-38, 2000.

[73] L. Smith and P. Pothakamuri, "The Effect of Surface Treatment of the Degradation of Composite Adhesives," in Joint Advances Materials and Structures (JAMS) Technical Review Meeting, Wichita, KS., 2005.

[74] E. Knox and M. Cowling, "A rapid durability test method for adhesives," International Journal of Adhesion and Adhesives, vol. 20, pp. 201-208, 2000.

[75] I. Ashcroft, D. Hughes and S. Shaw, "Adhesive Bonding of Fibre Reinforced Polymer Composite Materials," Assembly Automation, vol. 20, no. 2, pp. 150-161, 2000.

[76] S. Chaterjee, D. Adams and D. Oplinger, "Test Methods for Composites- A Status Report Volume III: Shear Test Methods," U.S. Department of Transportation DOT/FAA/CT-93/17, *III, Atlantic City International Airport, NJ, June 1993.

[77] T. Yokoyama and K. Nakai, "Interlaminar and In-Plane Shear Strengths of a Unidirectional Carbon/Epoxy Laminated Composite under Impact Loading," in Society for Experimental Mechanics (SEM), St. Louis, MO, 2006.

[78] R. Vodicka, B. Nelson, J. van den Berg and R. Chester, "Long-Term Environmental Durability of F/A-18 Composite Material," DOD Defence Science \& Technology Organization (DSTO) AMRL, Melbourne, Australia, 1999.

[79] M. Jastrezebski, A. Sinclair, J. Raizenne and J. Spelt, "Development of Adhesive Bonds with Reduced Fracture Strength as NDE Benchmarks," International Journal of Adhesion and Adhesives, vol. 29, pp. 372-379, 2009.

[80] N. Datlaa, J. Ulincy, B. Carlson, M. Papini and J. Spelt, "Mixed mode fatigue behavior of degraded toughened epoxy adhesive joints," International Journal of Adhesion and Adhesives, vol. 31, pp. 88-96, 2011.

[81] K. Lee and D. Lee, "Smart cure cycles for the adhesive joint of composite structures at cryogenic temperatures," Composite Structures , vol. 86, pp. 37-44, 2008.

[82] W. Johnson, L. Butkus and R. Valentin, "Applications of Fracture Mechanics to the Durability of Bonded Composite Joints," U.S. Department of Transportation DOT/FAA/AR-97/56, Washington, DC, May 1998. 
[83] A. Kinloch, M. Little and J. Watts, "The Role of the Interphase in the Environmetnal Failure of Adhesive Joints," Acta Materialia, vol. 48, pp. 4543-4553, 2000.

[84] R. Bhargava, S.-Q. Wang and J. L. Koenig, "FTIR Microspectroscopy of Polymeric Systems," Journal of Advanced Polymer Science, vol. 163, pp. 137-191, 2003.

[85] M. G. González, J. C. Cabanelas and J. Baselga, "Applications of FTIR on Epoxy Resins Identification, Monitoring the Curing Process, Phase Separation and Water Uptake," in Infrared Spectroscopy-Materials Science, Engineering and Technology, Croatia, European Union, InTech, April 2012, pp. 261-284.

[86] S. Cotugno, G. Mensitieri, P. Musto and L. Sanguigno, "Molecular interactions in and transport properties of densely cross-linked networks: A time-resolved FT-IR spectroscopy investigation of the epoxy/H2O system," Macromolecules, vol. 38, no. 3, pp. 801-811, Feb 2003.

[87] W. Chen, Y. Yu, P. Li, C. Wang, T. Zhou and X. Yang, "Effect of new epoxy matrix for T800 carbon fiber/epoxy filament wound composites," Composite Science and Technology, vol. 67, pp. 2261-2270, Feb 2007.

[88] A. Tracey and B. Flinn, "Infrared Spectroscopy: A Potential Quality Assurance Method for Composite Bonding Surface Preparation," in Joint Advances Materials and Structures (JAMS), Baltimore, MD, 2012.

[89] D. Fata and W. Possart, "Aging Behavior of a Hot-Cured Epoxy System," Journal of Applied Polymer Science, vol. 99, no. 5, pp. 2726-2736, 2006.

[90] B. Flinn, A. Tracey and T. Howie, "Composite Thermal Damage Measurement with Handheld FTIR," in Joint Advanced Materials and Structures (JAMS) Presentation, Mukilteo, WA, April 2013.

[91] F. Boerio, B. Roby and R. Dillingham, "Effect of Surface Engineering Processes on Surface Properties and Adhesive Bonding of Graphite/Epoxy Composites," in Proceedings of the 39th International SAMPE Technical Conference, 2007.

[92] N. Hameed, S. Thomas, R. Abraham and S. Thomas, "Morphology and contact angle studies of poly(styrene-co-acrylonitrile) modified epoxy resin blends and their glass fibre reinforced composites," Express Polymer Letters, vol. 1, no. 6, pp. 345-355, 2007.

[93] T. Yasuda, T. Okuno and H. Yasuda, "Contact Angle of Water on Polymer Surfaces," Langmuir, vol. 10, pp. 2435-2439, 1994. 
[94] G. Dillingham, B. Oakley, E. Dan-Jumbo, J. Baldwin, R. Keller and J. Magato, "Surface treatment and adhesive bonding techniques for repair of high-temperature composite materials," Journal of Composite Materials, vol. 0, no. 0, pp. 1-7, April 2013.

[95] G. Dillingham, B. Oakley, P. Van Voast, P. Shelley, R. Blakley and C. Brent Smith, "Quantitative Detection of Peel Ply Derived Contaminants via Wettability Measurements," Journal of Adhesion Science and Technology, vol. 26, no. 10-11, pp. 1563-1571, 2012.

[96] K. Armstrong, "Effect of absorbed water in CFRP composites on adhesive bonding," International Journal of Adhesion and Adhesives, vol. 16, pp. 21-28, 1996.

[97] P. Hintikka, M. Wallin and O. Saarela, "The effect of moisture on the interlaminar fracture toughness of CFRP laminate," in 27th International Congress of the Aeronautical Sciences, Nice, France, 2010.

[98] A. Ameli, N. Datla, M. Papini and J. Spelt, "Hygrothermal Properties of Highly Toughened Epoxy Adhesives," The Journal of Adhesion, vol. 86, no. 7, pp. 698-725, 2010.

[99] X. Jiang, H. Kolstein and F. S. Bijlaard, "Moisture Diffusion in FRP Adhesively-Bonded Joints under Hot/Wet Environments," in CICE 2010 - The 5th International Conference on FRP Composites in Civil Engineering, Beijing, China, Sep 2010.

[100] J. Ryan, R. Adams and S. Brown, "Moisture Ingress Effect on Properties of CFRP," in 17th Int. Conf. on Composite MaterialS, Edinburgh, UK, 2008.

[101] C. Shen and G. Springer, "Noisture Absorption and Desorption of Composite Materials," Journal of Composite Materials, vol. 10, pp. 2-20, 1976.

[102] B. Parker, "Some Effects of Moisture on Adhesive Bonded CFRP-CFRP Joints," Composite Structures, vol. 6, pp. 123-139, 1986.

[103] E. O'Brien, Durability of adhesive joints subjected to environmental stress, Blacksburg, VA: Dissertation for Doctor of Philiosophy; Virginia Polytechnic Institute, 2003.

[104] T. Nguyen, E. Byrd, D. Alsheh, W. McDonough and J. Seiler, "Interfacial Water and Adhesion Loss of Polymer Coatings on a Siliceous Substrate," Materials Research Society, vol. 385, pp. 57-63, 1995.

[105] F. Bowden and W. Throssell, "Adsorption of Water Vapour on Solid Surfaces," Nature, vol. 167, pp. 601-602, April 1951. 
[106] R. Selzer and K. Friedrich, "Influence of water up-take on interlaminarfracture properties of carbon fibre-reinforced polymer composites," Journal of Materials Science, vol. 30, pp. 334-338, 1995.

[107] Y. Arao, J. Koyanagi, H. Hatta, Y. Aoki and H. Kawada, "Effect of moisture absorption on dimensional stability in carbon/epoxy composites," in 16th International Conference on Composite Materials, Kyoto, Japan, 2007.

[108] L. Silva, "Special Issue on Durability of Adhesive Joints," International Journal of Adhesion and Adhesives, vol. 29, pp. 593-594, 2009.

[109] M. Davis and D. Bond, "Principles and practices of adhesive bonded structural joints and repairs," International Journal of Adhesion and Adhesives, vol. 19, pp. 19-105, 1999.

[110] T. Pribanic, D. McDaniel, V. Musaramthota, L. Sanchez, N. Munroe, X. Zhou, J. Zhou and S. Cai, "Development of Durability Test Procedure for Adhesively Bonded Composite Joints," in SAMPE, Baltimore, MD, May 22-24, 2012.

[111] N. Datla, M. Papini, J. Ulicny, B. Carlson and J. Spelt, "The effects of test temperature and humidity on the mixed-mode fatigue behavior of a toughened adhesive aluminum joint," Engineering Fracture Mechanics, vol. 78, no. 6, April 2011.

[112] H. Kim, C. Sun and T. Siegmund, "Damage Tolerance and Durability of Adhesively Bonded Composite Structures," in Joint Advanced Materials and Structures (JAMS), Seattle, WA, 2006.

[113] H. Liu, A. Uhlherr, R. Varley and M. Bannister, "Influence of substituents on the kinetics of epoxy/aromatic diamine resin systems," Journal of Polymer Science Part A: Polymer Chemistry, vol. 42, no. 13, pp. 3143-3156, July 2004.

[114] R. J. Varley, W. Liu and G. P. Simon, "Investigation of the Reaction Mechanism of Different Epoxy Resins Using a Phosphorus-Based Hardener," Journal of Applied Polymer Science, vol. 99, no. 6, pp. 3288-3299, Jan 2006.

[115] J. Mijovic, A. Fishbain and J. Wijaya, "Mechanistic modeling of epoxy-amine kinetics. 1. Model compound study," Macromolecules, vol. 25, no. 2, pp. 979-985, 1992. 


\section{MATERIALS AND MANUFACTURING}

This chapter provides all the detailed information on the materials utilized for experimental analysis such as adhesives, adherends and contaminants. A brief set of manufacturing procedures adopted will also be explained.

\subsection{Materials}

Specimens for this study have been manufactured with composite prepregs. The initial studies utilized unidirectional carbon epoxy prepreg that was acquired from Toray Composites America (TCA). The material was a unidirectional prepreg T800 (P2362U-19-304) with a fiber areal weight (FAW) of $190 \mathrm{~g} / \mathrm{m}^{2}$ and resin content (RC) of approximately 35\%. The adhesive utilized was 3M Scotch-Weld (AF 555 U \& AF 555 M). The U indicates unsupported tape and the M indicates that the tape includes a supported mesh. The Toray prepreg is a semi toughened proprietary epoxy prepreg system which cures at $350^{\circ} \mathrm{F}$ with a young's modulus of $22.3 \times 10^{6} \mathrm{psi}$ and $60 \%$ fiber volume. Both AF 555 adhesives are designed to cure at $350^{\circ} \mathrm{F}$ for bonding composites. This adhesive can be co-cured, and co-bonded with composite prepreg, or used to bond cured composites.

Peel ply materials acquired from FibreGlast consisted of both and nylon and polyester 583 peel plies. Additional polyester ply, P60001, was obtained from Precision Fabrics. These peel plies are used to protect the composites prior to bonding and to generate an active surface when removed. Peel plies are often used in secondary bonding applications.

Various types of contamination were used in this study including an aerosol from CBS Aerosol \& Paint, Inc. and Frekote 710-NC from Henkel Corporation. The aerosol is a 100\% Silicone spray and the Frekote is a semi-permanent proprietary release agent. As per the data sheet, the Frekote contains alkanes, octanes, naphtha, dibutyl ether and polydimethylsiloxane (PDMS) which is one of the most common contaminants that can effectively release substrates. Initial testing 
with the aerosol proved to be unreliable so efforts focused on using the Frekote due to its popularity as a release agent. The PDMS molecular structure within Frekote is given in Figure 3.1 below.

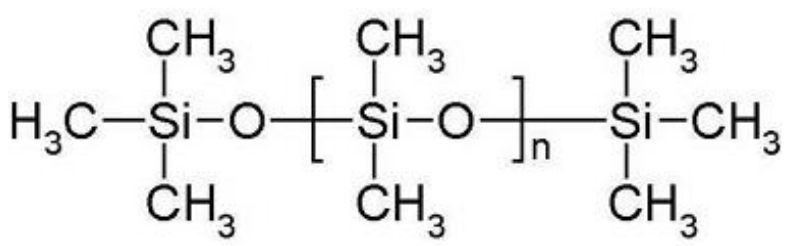

Figure 3.1: Molecular Structure of PDMS [1]

\subsection{Composite Laminate Design}

A well designed composite laminate will exhibit characteristics such as excellent strength and stiffness to weight ratios, good fatigue properties and tuning the stiffness and strength to meet the end result of design requirements. To simplify analysis, testing and manufacturing, the primary design suggests that a composite laminate be made symmetric. Non-symmetric laminates introduces coupling and can affect the stiffness of the laminate. Any asymmetries that are essential should be kept at the middle surface where the adhesive is introduced. The database for composite materials is fiber and matrix specific and extrapolating them to new materials is risky. The material system that was utilized here is designed based on the factors aforementioned and described in section 3.3.

\subsection{Manufacturing of Adhesively Bonded Composite Laminates}

In this research, DCB coupons are manufactured as per the ASTM D5528 standard via a sequence of steps that are explained in detail below. An end product of a composite system depends on its manufacturing processes adopted. The process is based on the applicability and economic value. A wide variety of manufacturing techniques exist such as compression molding (CM), liquid composite molding (LCM), injection molding (IM), reinforced reaction injection molding (RRIM), structural reaction injection molding (SRIM), resin transfer molding (RTM), vacuum assisted resin transfer molding (VARTM), vacuum infusion processing (VIP) and hand layup to name a few [2]. 
Advanced composite materials typically comprise of thermosets and require several cure steps to attain desired properties on an end product. The manufacturing of these composite laminates primarily starts with a hand lay-up process. A hand layup process was preferred in this research as our production requirements are minimal. Dry prepreg plies are cut into desired dimensions as shown in Figure 3.2 (a) and are layed by hand on to a smooth finished plate to form a laminate stack as shown in Figure 3.2 (b). The continuous roll of prepreg sheet/ply is protected by a backing material which is subsequently removed as each layup step progresses. The fiber layers or plies in a laminate are arranged unidirectionally which is the primary loading
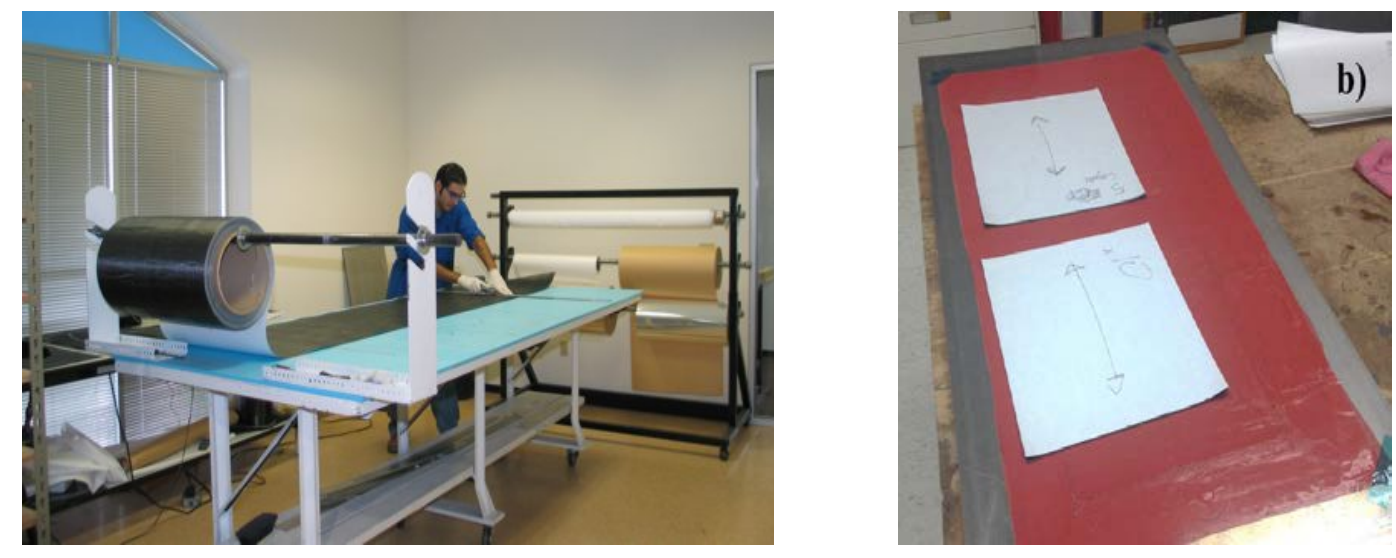

Figure 3.2: a) Dry prepreg laminate sheets cut to the desired dimensions and b) Stack of 10 plies of prepreg laminate.

direction. The sequence and symmetry has to be maintained in order to avoid misalignment of the layers post cure. Unidirectional fibers typically offer higher stiffness and are stronger in the fiber direction (here 0 degree). Stacking of the prepreg plies to a desired number, based on the thickness needed for of final laminate is then conducted. The red sheet shown in Figure 3.2b underneath the stacked laminates is the release ply which acts as a barrier between the laminate and the tool plate. This layer usually extends beyond the edges of the layup and is sealed with the help of a high temperature tape or a flashbreaker (FB) tape.

After the laminate stack is arranged, they are covered with the peel ply. The dimensions of peel ply are usually an inch over the actual laminate dimension as shown in Figure 3.3(a). The FB 
tape utilized is a high temperature tape that can sustain maximum temperatures of $400^{\circ} \mathrm{F}$ as well as holding down vacuum bagging materials. The peel ply fabric is applied on both sides of the laminate and sealed with the FB tape as shown in Figure 3.3. The breather material is then covered across the entire area of cure. The breather material absorbs excessive resin from the laminate and also maintains a path throughout the cure, so that air and volatiles can escape.

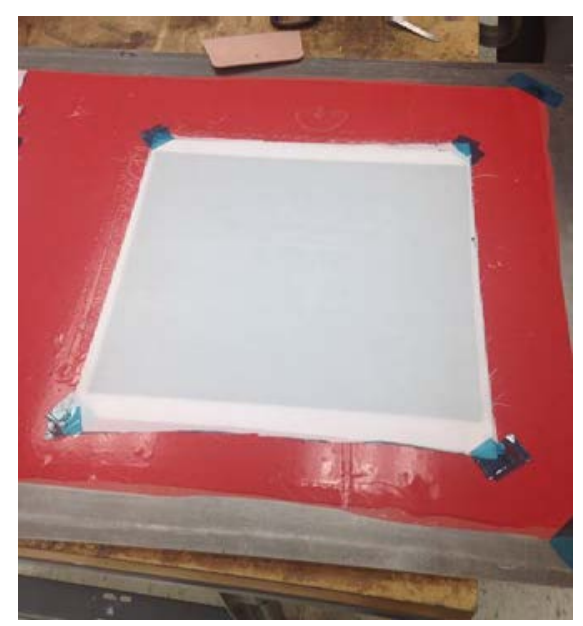

(a)

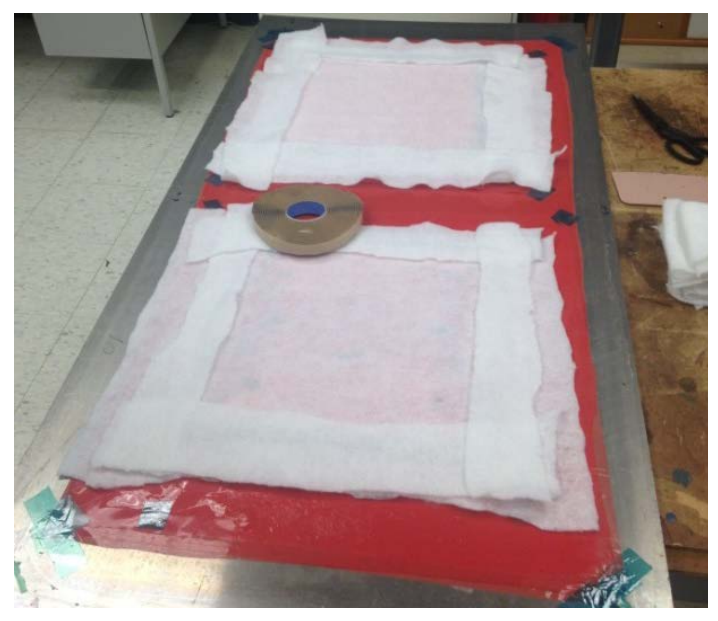

(b)

Figure 3.3: a) Application of Peel ply fabric on to the stacked laminate; b) breather material on top of peel ply covered stacked laminate.

Several layers of breather material can be utilized in case of heavy resin bleeds. This is shown in Figure 3.3(b). A rubberized sealant tape is utilized to cover the periphery of the tool plate so that it creates a circumferential boundary. Vacuum valves, ports and thermocouples are placed accordingly to monitor the vacuum levels inside the bag and to record temperatures of the part. A vacuum bag is then placed on top of the sealant tape to seal the edges and ensuring no leaks. These are generally nylon or polyvinyl alcohol (PVA) materials and is shown in Figure 3.4. The compaction pressure provided by the vacuum bag governs the quality of the composite laminates that are to be cured. In autoclave curing, although higher pressures can be attained to provide sufficient compaction pressure, vacuum bagging is still needed to outgas the available gases in the 
clave as well as reduce porosity that can be achieved in the absence of vacuum bag. Figure 3.4 provides and an image of the composite prior to being placed in an autoclave.

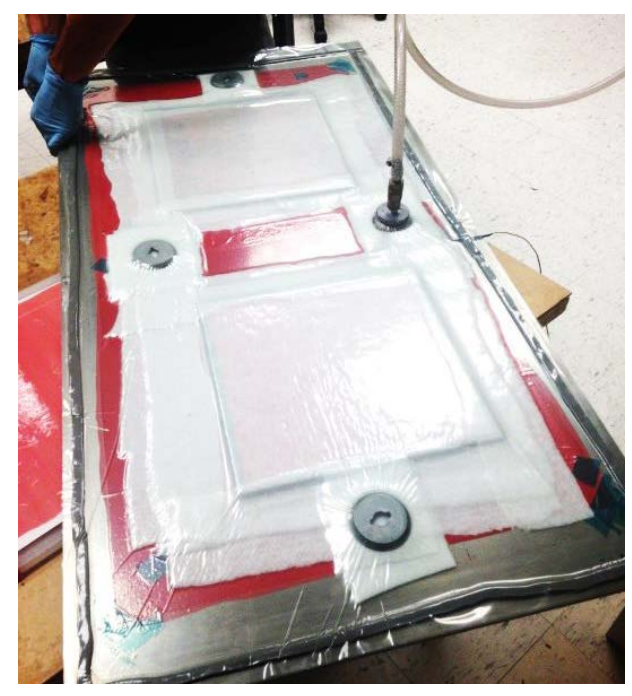

Figure 3.4: Vacuum applied to the vacuum bagging process

A schematic showing a cross section of the set-up is provided in Figure 3.5 and the list of items that are utilized are shown in Table 3.1. After the bagging is completed, the sealed plate is placed in an autoclave for curing. Autoclave is widely used in aerospace industry for the manufacturing of aircraft components, spacecraft and missiles. An autoclave is a pressurized chamber used to heat treat specimens under various conditions of temperature and pressure. This is conducted in accordance with cure cycles recommended by the manufacturer. With the high temperatures and pressures that can be achieved in the autoclave, a denser, composite part with minimal voids can be obtained. The autoclave used for our manufacturing is shown in Figure 3.6. The interior of an autoclave has many thermocouple ports, vacuum source ports and vacuum transducer ports. The cure process is typically provided by the prepreg manufacturer and must be followed to obtain high quality parts. The heating rate, pressure application rate, part temperature along with the cooling rate is usually specified with tolerance limits. Deviations from these requirements will lead to lower quality parts. Figure 3.7 shows the cure cycle of panels made with the T800 prepreg. The chart shows measurements 


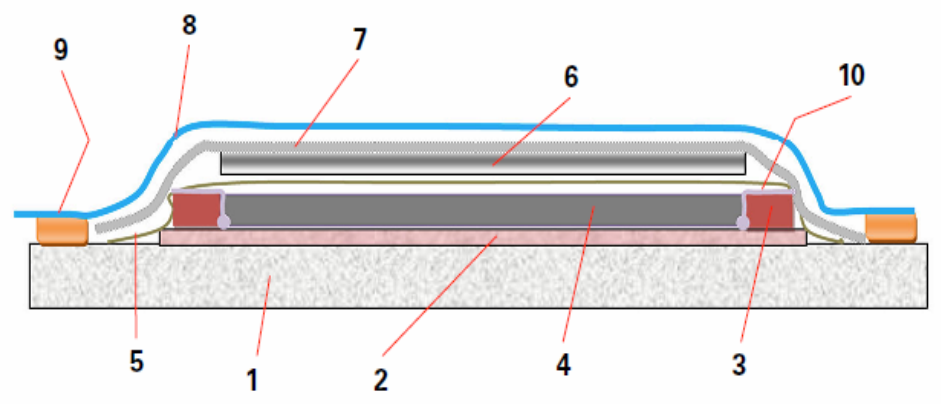

Figure 3.5: Schematic showing the vacuum bagging procedure setup.

Table 3.1: List of Materials utilized during composite laminate manufacturing

\begin{tabular}{|c|c|c|}
\hline & \multicolumn{2}{|c|}{ List of Materials } \\
\hline 1 & Tool & Aluminum (release film covered) \\
\hline 2 & Release film & FibreGlast \\
\hline 3 & Silicon edge dams & Thicker than laminate \\
\hline 4 & Laminate & As fabricated \\
\hline 5 & Release film & FibreGlast \\
\hline 6 & Caul plate & Aluminum, Steel or Invar \\
\hline 7 & Breather & 2.2 osy polyester breather \\
\hline 8 & Vacuum bag & FiberGlast \\
\hline 9 & Vacuum sealant & \\
\hline 10 & Glass yarn string & NA \\
\hline & & \\
\hline
\end{tabular}

such as temperature, pressure, vacuum, part temperature, vessel temperature etc, as a function of time for the cure cycle. The vacuum lines and the pressure lines overlap each other in the cure cycle chart which indicates that there was no major leak. The autoclave air temperature leads the part temperature which is expected, and the air temperature always overshoots at the end of the ramp which was done intentionally and automatically by the autoclave controller, so that the parts can reach the cure temperature more quickly. The parts are cured for the desired time and temperature for an efficient bonding process and cooled down. In the process of optimizing parameters, effects of vacuum were investigated where vacuum was applied throughout the cure for one set while 
vacuum was switched off after the vessel pressure attained 12 psi. The parts are maintained under compression on the composite parts from here on and result in the desired thickness. Hence, for the manufacturing of our parts, the vacuum was removed at a vessel pressure of 12 psi.

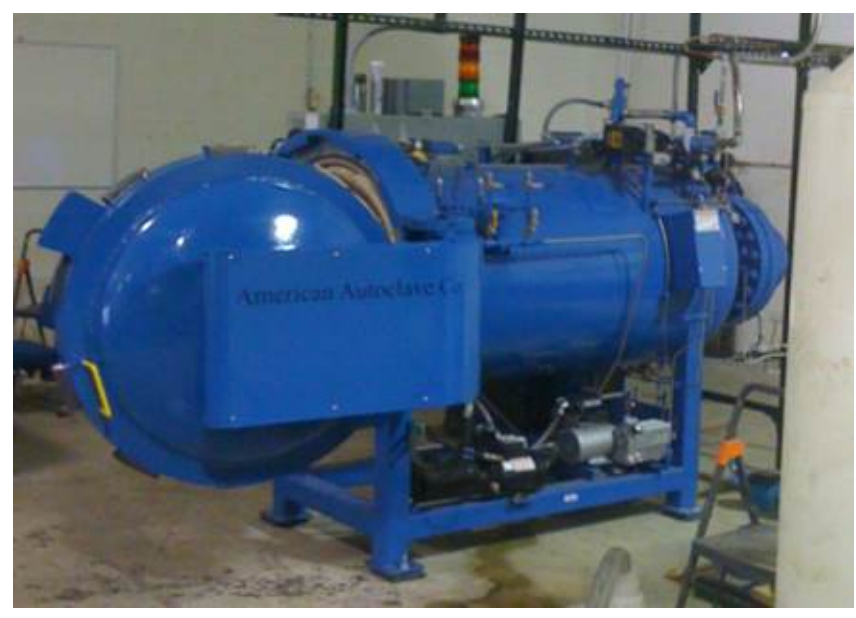

Figure 3.6: Image of an autoclave utilized for composie manufacturing

\section{Toray- AF 555M bonded system}

The laminates were cured at $350^{\circ} \mathrm{F}$ in accordance with the manufacturer's specifications. Secondary bonding was performed at $350^{\circ} \mathrm{F}$ for 2 hours. The laminates measured approximately 13.5 inches in length by 12 inches in width. Two 10 ply laminates with all $0^{\circ}$ ply orientation and two supported mesh adhesive films were used as the adhesive to bond the panels (the choice of number of plies and adhesives are detailed later in this section). A precrack of 2.5 inches for easy crack initiation was embedded with a release film in between the adhesive layers. After the bonding process was accomplished, 8 specimens were cut from each panel and measured 11.5 inches in length by 1 inch in width. Remaining material from each panel was utilized in the surface characterization studies. The number of plies was determined based on the mechanical properties of the adhesive. Table 3.2 shows the fatigue properties of AF 555. Maximum loads at the surfaces and required forces to cause the desired shear stress at the bond line were calculated. 


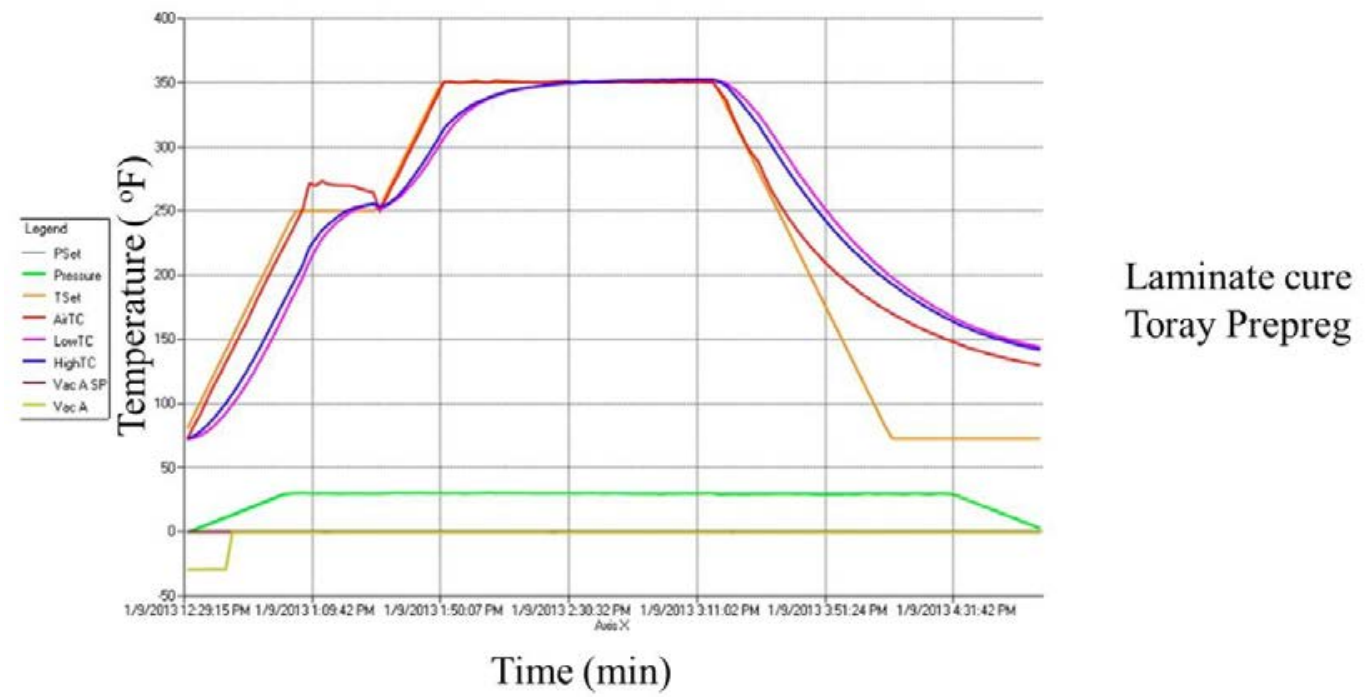

Figure 3.7: Cure cycle for Toray Prepreg

Table 3.2: Maximum Stress and Force Calculations for Toray

\begin{tabular}{|c|c|c|}
\hline Max Stress (psi) & Average Life (cycles) & $\begin{array}{c}\text { Force required for a 20 ply 9 inch } \\
\text { long specimen }\end{array}$ \\
\hline 4500 & $1.58 \times 104$ & - \\
\hline 4000 & $5.28 \times 104$ & - \\
\hline 3500 & $4.75 \times 105$ & 420 \\
\hline 3000 & $2.67 \times 106$ & 360 \\
\hline 2200 & $1.03 \times 107+$ (No failure) & 264 \\
\hline
\end{tabular}

Table 3.3 provides the displacement and force requirements as a function of number of plies. These parameters were obtained by assuming a 3000 psi shear load at the bondline. Based on this table, the specimen design resulted in 20 plies of a unidirectional laminated ( 0.15 in thick + adhesive) with a reasonable force load of $300 \mathrm{lbs}$ and a displacement of 1.07 inches without failure. Deflections were determined using Castigliano’s theorem,

$$
\omega=\frac{F l^{3}}{3 E I}
$$

where F is the load, $\mathrm{l}$ is the length, $\mathrm{E}$ is the Young's Modulus and I is the Inertia. Specimens were tested to deflections of $0.25,0.5$ and 0.75 inches with $80 \%$ of tested specimens fracturing at the 0.75 in deflection. Hence, the 0.5 inch of single amplitude was selected as the appropriate 
Table 3.3: Displacement and Force Dependency on Number of Plies

\begin{tabular}{|c|c|c|c|c|c|}
\hline 3000 & \#Plies & Thickness (t) & Inertia (I) & Force on Piston (F) & Stress at Surface $(\sigma)$ \\
\hline & 2 & 0.015 & $2.81 \mathrm{E}-07$ & 30 & 1800 \\
\hline & 4 & 0.03 & $2.25 \mathrm{E}-06$ & 60 & 900 \\
\hline & 6 & 0.045 & $7.59 \mathrm{E}-06$ & 90 & 600 \\
\hline $\mathrm{T}=3000$ & 8 & 0.06 & $1.80 \mathrm{E}-05$ & 120 & 450 \\
\hline & 10 & 0.075 & $3.52 \mathrm{E}-05$ & 150 & 360 \\
\hline & 12 & 0.09 & $6.08 \mathrm{E}-05$ & 180 & 300 \\
\hline & 14 & 0.105 & $9.65 \mathrm{E}-05$ & 210 & 257 \\
\hline & 16 & 0.12 & $1.44 \mathrm{E}-04$ & 240 & 225 \\
\hline & 18 & 0.135 & $2.05 \mathrm{E}-04$ & 270 & 180 \\
\hline & 20 & 0.15 & $2.81 \mathrm{E}-04$ & 300 & 164 \\
\hline & 22 & 0.165 & $3.74 \mathrm{E}-04$ & 330 & 150 \\
\hline & 24 & 0.18 & $4.86 \mathrm{E}-04$ & 360 & \\
\hline
\end{tabular}

deflection parameter. The curing time of the AF 555 adhesive is 2 hours at $350^{\circ} \mathrm{F}$ as per manufacturer's recommendations for bonding to composite. The adhesive cure profile is shown in Figure 3.8.

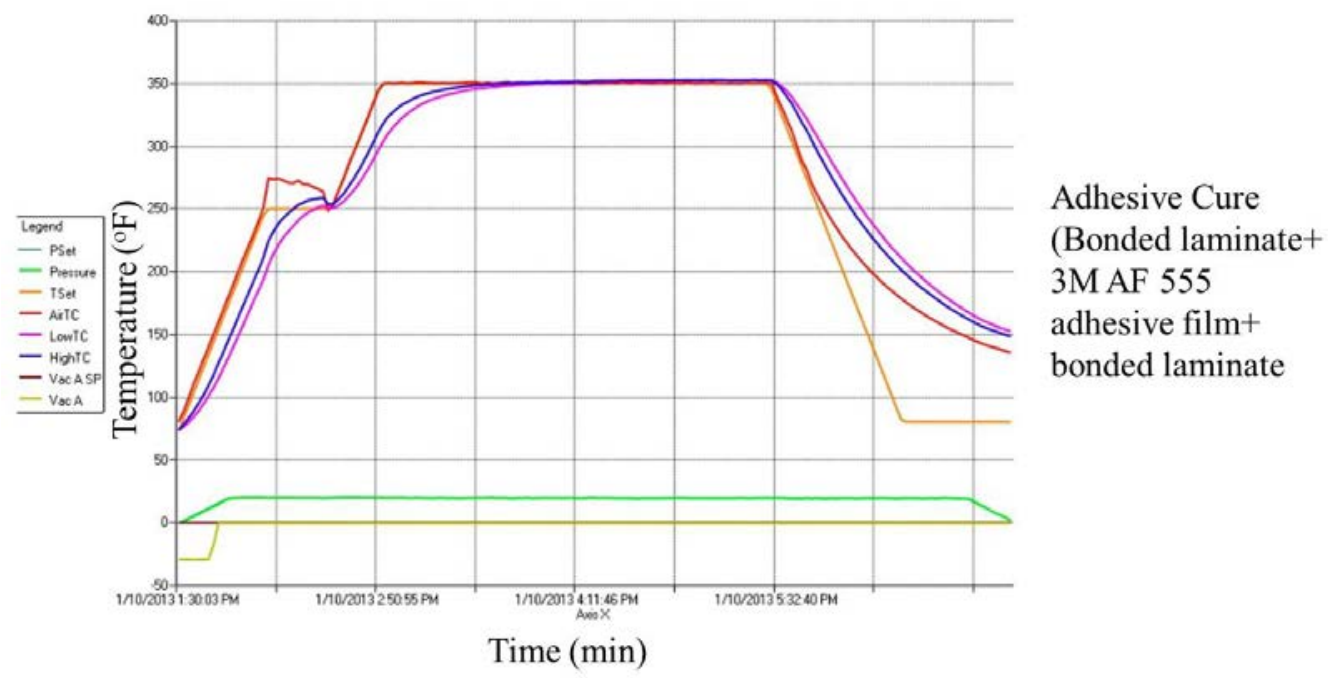

Figure 3.8: Adhesive cure cycle of bonded composite laminates

3.4 Preparation of Contaminated Adhesively Bonded Composite Laminates 
Durability of the ABCJ's can potentially be affected from contamination on the laminate surfaces, which can degrade the bond quality/bond strength over time. To study the effect on durability a procedure for contaminating the laminate surfaces prior to bonding is needed. The objective is to create weaker links between the adherend and adhesive and create a means for studying the effects of contamination on bond durability. Several materials were evaluated including grease, vacuum oils, silicon gels and release agents. It was found that release agents were more effective in providing repeatable and reliable results. Release agents are lubricants that are often applied to mold surfaces to facilitate release of the molded article. This creates a controlled adhesion that can generate scalable and repeatable bonding conditions. This was shown by systematically controlling the amount of contamination created or deposited on the laminate surface.

\subsubsection{Contamination Methods}

The aim of developing a procedure to contaminate the laminate surfaces prior to bonding is to create weaker links between the adherend and adhesive and determine the effect of contamination on bond durability in ABCJ's. Several approaches were explored at FIU to create scalable and repeatable bonding conditions. This was exercised by systematically controlling the amount of contamination created or deposited on the laminate surface. The contaminant employed initially was $100 \%$ pure Silicone spray (CBS Aerosol \& Paint, Inc). Two approaches were finalized to investigate their effect on bond strength. They are detailed below.

\subsubsection{Mesh Contamination}

For this approach, a perforated stainless steel mesh $(0.305 \mathrm{~m}$ x $0.305 \mathrm{~m})$ containing equipatterned holes with a diameter of $3.2 \mathrm{~mm}$, thickness of $0.91 \mathrm{~mm}$ and a stagger of $22.2 \mathrm{~mm}$ was utilized. The mesh was vertically placed at a $90^{\circ}$ angle and the contaminant was sprayed in the perpendicular direction towards the mesh as shown in the Figure 3.9. Care was taken to create a uniform layer of contaminant all across the perforated mesh. The mesh containing the contaminant 
side is brought in contact with the laminate surface to be bonded to replicate the exact same pattern as that of mesh. This creates a contaminant imprint on the laminate surface. It should be noted that gravimetric analysis was conducted on the laminates before and after contamination in order to record the amount of contaminant deposited.
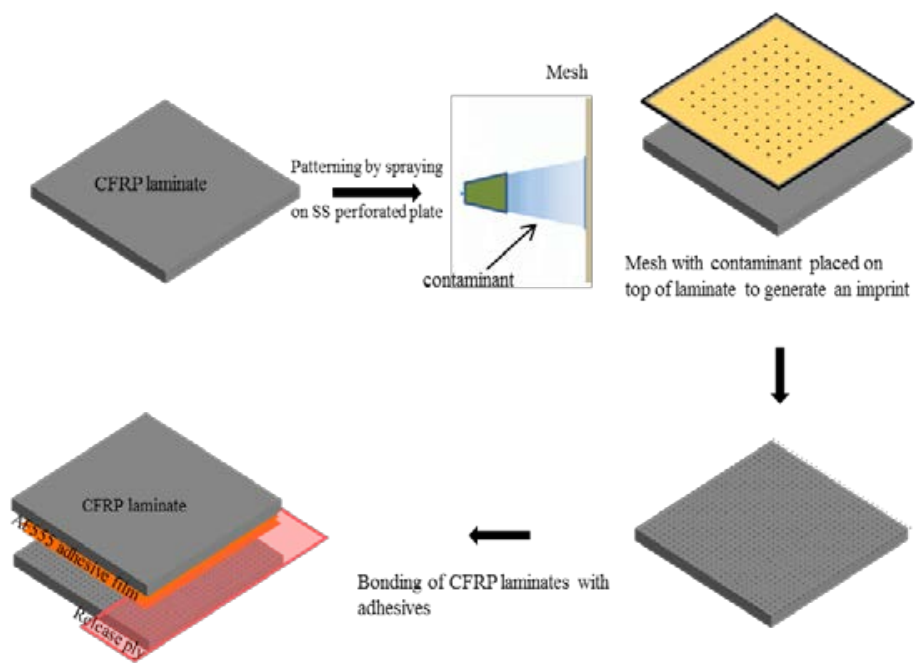

Figure 3.9: Contamination Procedure- Mesh Approach

\subsubsection{Stamp Contamination}

In the stamp approach, a rubber stamp with a dotted pattern is brought in contact with the contaminant. Arbitrary arrays of spatially ordered patterns of macroscopic feature size are created on the surface of the laminate. The contaminant is transferred onto the stamp following which the inked contaminant stamp is brought in contact with the laminate surface as shown in Figure 3.10. This generates an imprint on the laminate surface with a high throughput than mesh approach. As mentioned above, gravimetric analysis was performed on laminates before and after contamination in order to monitor the weight gains. The patterned imprint on a composite surface can be viewed in the bottom portion of the Figure 3.10. This results in the generation of a contaminant pattern that creates the same surface information as of its stamp. This technique is versatile and can become increasingly useful as it enables the fabrication/manufacturing process 


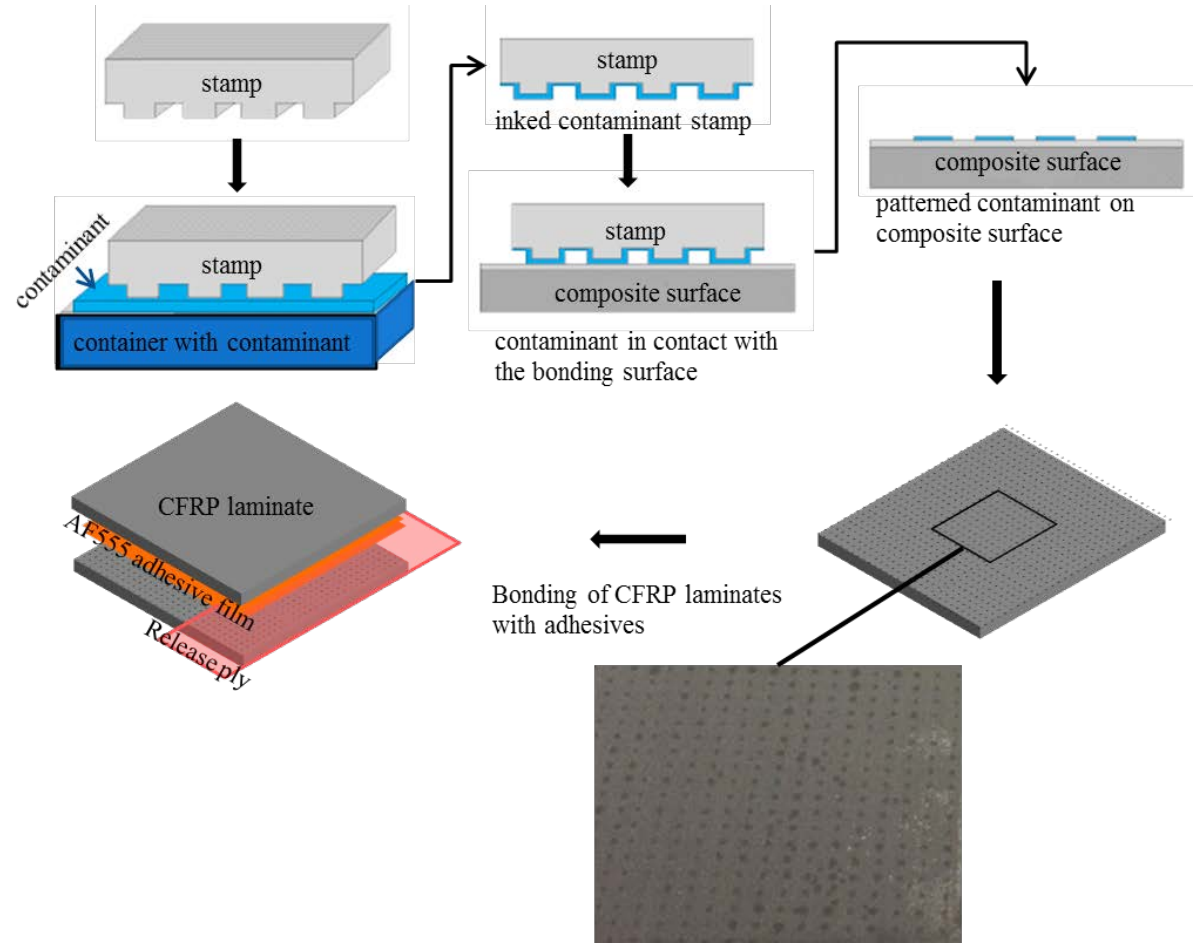

Figure 3.10: Contamination Procedure- Stamp Approach

very cost effective and by creating localized contaminant sites efficiently. In both the approaches, the spatially ordered patterns can be varied by altering the diameter of the holes on the mesh or varying the dotted pattern on the stamp. Once the approach is optimized, we then followed the utilization of different contaminants to observe their effect on bond strength.

\subsubsection{Contaminant Materials}

The two contamination procedures were evaluated in conjunction with contaminant type in an effort to optimize a contaminant that will successfully create a weak link at the interface or between the adhesive and the composite surface. Several contaminants were utilized to contaminate the composite surface and evaluations of its effect on bond strength were conducted. The contaminant types used are mentioned as follows:

\subsubsection{Aerosol Contaminant}

The $100 \%$ Silicone spray was used to evaluate both the mesh and stamp approaches for contaminating the laminates. The approaches were investigated to determine which approach 
provided more consistent contamination patterns. As shown in Figure 3.11, the mesh approach was significantly less consistent in depositing the contamination. Subsequent contamination in this study was done with the stamp approach.
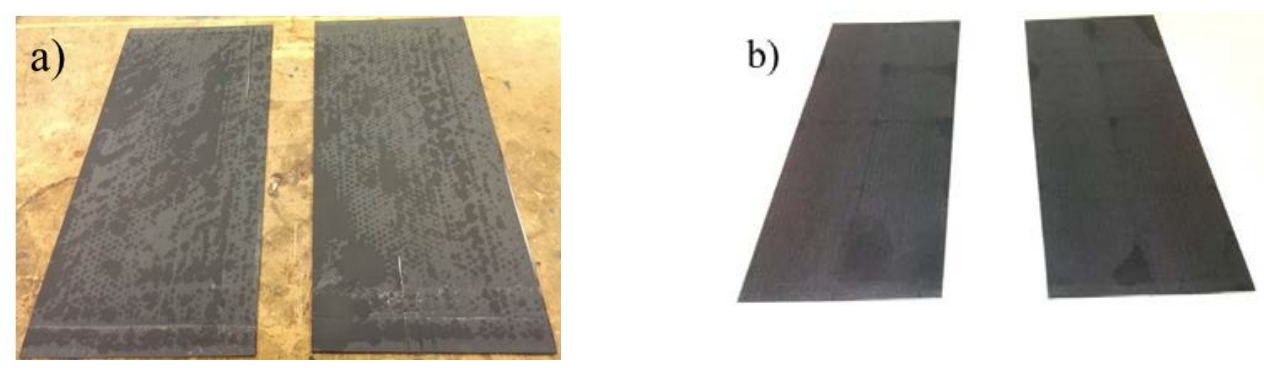

Figure 3.11: Differences in the type of approaches a) Mesh; and b) Stamp

\subsubsection{Frekote Contaminant}

Frekote, another contaminant utilized to study the effect of contamination on bond strength. Frekote is a semi-permanent mould release agent and no research on the effect of bond strength using composite materials has been reported so far. The company data sheet reveals the presence of polydimethylsiloxane (PDMS), dibutyl ether, light aliphatic solvent naphtha and isoparaffinic hydrocarbon. Upon researching, frekote contains alkanes, octanes, naphtha, dibutyl ether and polydimethylsiloxane (PDMS). It was also reported as one of the most common contaminants that can effectively release substrates from any adherends. Additionally frekote has gained popularity as an effective release agent. Frekote contaminant via a stamp approach yielded a better consistence in comparison to aerosol.

\subsubsection{Contamination Symmetry}

Efforts were also made to observe the effect of contamination by applying contaminant stamped on both sides of the composite laminate prior to bonding and only on a single side. The aim of creating weak bonds is to drive the fracture towards the weakest link.

\subsubsection{Dual Side Contamination}

The method of application of contaminant on dual sides followed the exact stamp approach 
mentioned in section 3.4.1.2 and the contaminant was applied on both sides of the composite laminate. This case will tend to progress the crack in both directions having considering the symmetrical plies i.e. a jaggered pattern between one laminate to another. The distribution of weak sites might not be uniform with each laminate and this complicates the understanding of crack propagation.

\subsubsection{Single Side Contamination}

The development of single side contamination approach over dual side was motivated to simplify the crack propagation. The crack path always tends to follow in a direction towards the weakest link where the failure occurs in a no adhesion region, which is the contaminant side whereas the other side is pure cohesive. This scenario will aid in monitoring the crack propagation with ease discerning in a no adhesion region on single side in comparison to monitoring crack propagation in both sides.

\subsubsection{Contamination Levels}

With the single side stamp contamination technique, it is proposed that monitoring of fracture can easily be identified and simplifies the analysis. In this investigation, understanding how varying levels of contamination effect crack dependency on fracture toughness is critical. One

of the major obstacles arising in this technique is the control of the resultant feature size and how that dictates the non-bondability of an adhesive bond.

\subsubsection{Stamp with Small Area (A1)}

Stamp with the dotted pattern in which each raised imprint dot is $1 \mathrm{~mm}$ in diameter with a separation distance of $4.5 \mathrm{~mm}$ in the horizontal direction and $3 \mathrm{~mm}$ in the vertical direction was procured. A1 stamp measured $7 \mathrm{~cm}$ x $7.5 \mathrm{~cm}$ in dimensions. Upon contaminating with Frekote, this geometry results in a ratio of 5.43 percent contaminated area per unit cell. The A1 stamp is shown in Figure 3.12a.

\subsubsection{Stamp with Larger Area (A3)}


Stamp with the dotted pattern in which each raised imprint dot is $3 \mathrm{~mm}$ in diameter and an $8 \mathrm{~mm}$ of separation distance in both horizontal and vertical direction was procured. A3 stamp measured $6.5 \mathrm{~cm} \times 13 \mathrm{~cm}$ in geometry. After contamination, this geometry results in a ratio of 5.58 percent contaminated area per unit cell. The A3 stamp is shown in Figure 3.12b.

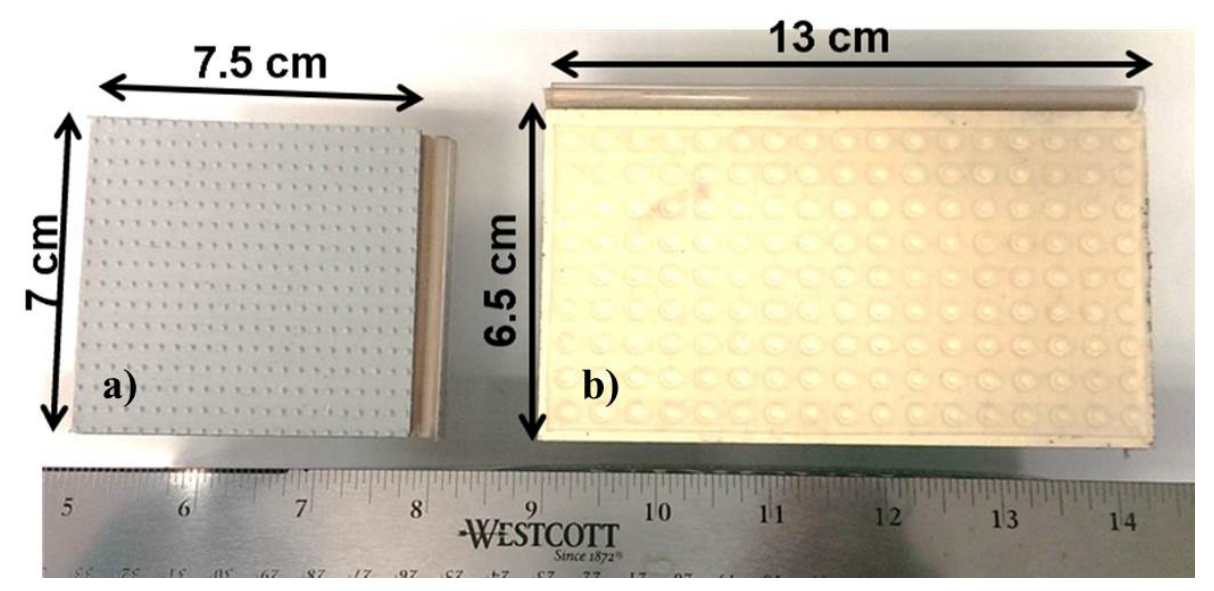

Figure 3.12: Comparison of A1 and A3 stamps

Using this approach, contaminant locations will create adhesion failure (failure at the composite-adhesive interface) that is intermixed with areas of cohesion failure (failure within the adhesive itself). By varying the size and application pressure of the stamp an improved understanding of the influence of each type of failure on overall bond strength can be assessed.

\subsection{Summary}

The contamination methods, materials and levels optimized would reveal a comprehensive understanding of weak links within an adhesively bonded joint. The primary advantage of the contamination method adopted would assist in creating a repeatable and scalable undesirable bonding conditions that are expected to serve as NDE benchmarks to further the understanding of effects of contamination in ABCJ's. 


\section{SURFACE CHARACTERIZATION}

Surface characteristics of any material are often controlled by the surface chemical and physical properties. Characterizing the surface features or any properties that are associated with the surface is of great importance because these surfaces are a primary mode of contact for the adhering material. Assessing the surface can reveal information such as activity, available chemical groups and surface features such as topography. This chapter describes available surface characterization techniques which can provide surface information which might serve as a benchmark in predicting the adhesion mechanisms involved with the bonded joints. Figure 4.1 illustrates the surface characterization techniques and tools that were utilized in the current research to analyze the properties and surface chemistry associated with composite laminates.

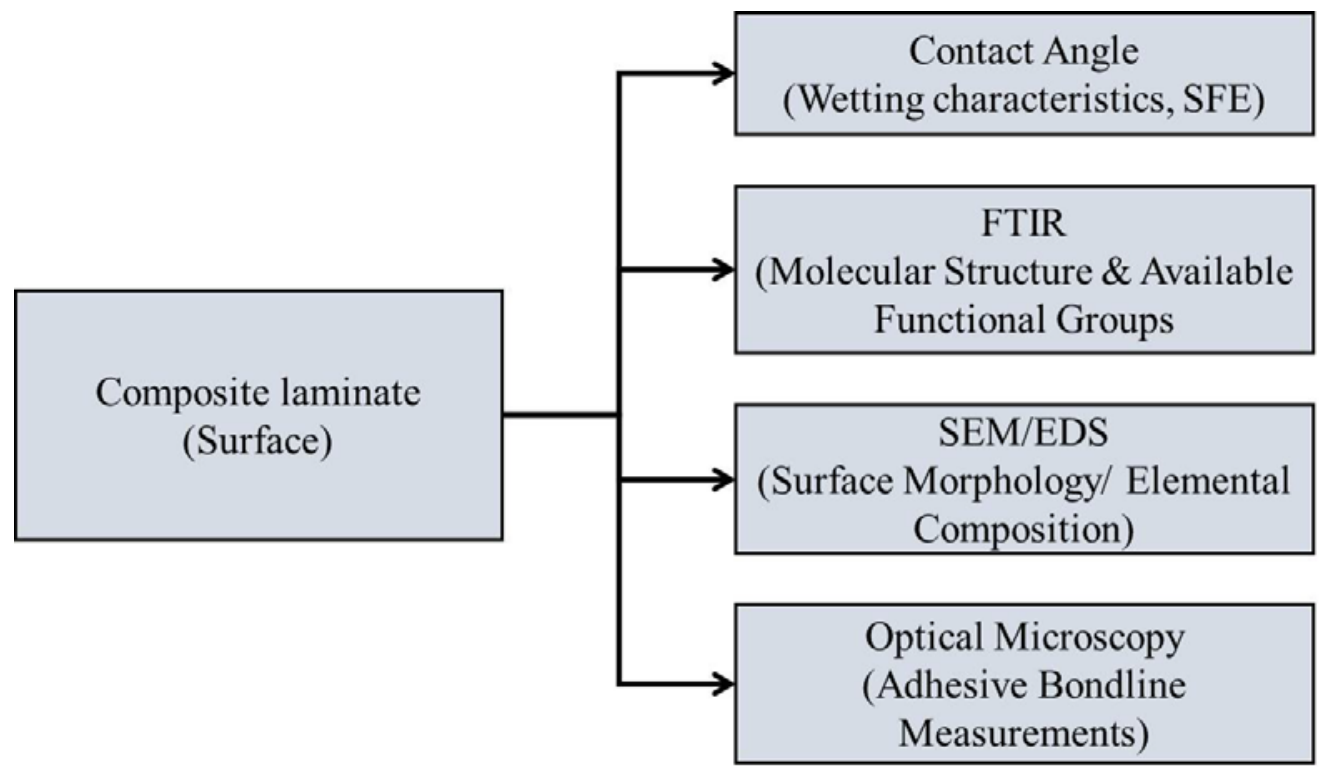

Figure 4.1: Surface characterization techniques conducted in the current research.

The information that can be acquired from utilizing these surface techniques are explained in detail in the following sections. It should be noted that many routes were adopted for contamination, of which a comparison is drawn between the pristine and the optimized contaminated set only in order to observe the effect of contamination on bond performance. 


\subsection{Experimental Approach}

\subsubsection{Wettability Measurements}

Contact angles were obtained using the sessile drop method with a KYOWA Contact angle meter (model no: DM-CE1) equipped with a dispensing needle as shown in Figure 4.2(a). Three liquids were utilized for measuring the contact angles: DI water which is mildly polar, ethylene glycol-neutral in nature and diiodomethane-highly polar in nature. An ideal probe liquid would be the adhesive itself which would give comprehensive information about the kinetics of the contact mechanism but the viscosities of the adhesive is extremely high and does not allow its use as probe liquids. Additionally, the polarity of the adhesive is unknown. This aids in the selection of known liquids having high, neutral and lower polarity as an alternative. A $2 \mu$ droplet (sessile) was generated by rotating the needle and approaching the substrate perpendicular to the needle direction with a gentle feed rate of a few micrometers per minute. All the tests were carried out in ambient air at room temperature. 10 drops for each liquid were analyzed at 10 different locations separated by sufficient spacing $(\sim 0.5 \mathrm{~mm})$ to prevent the potential influence of previous tests on the substrate. With the aid of FAMAS, an image analysis software, the shape
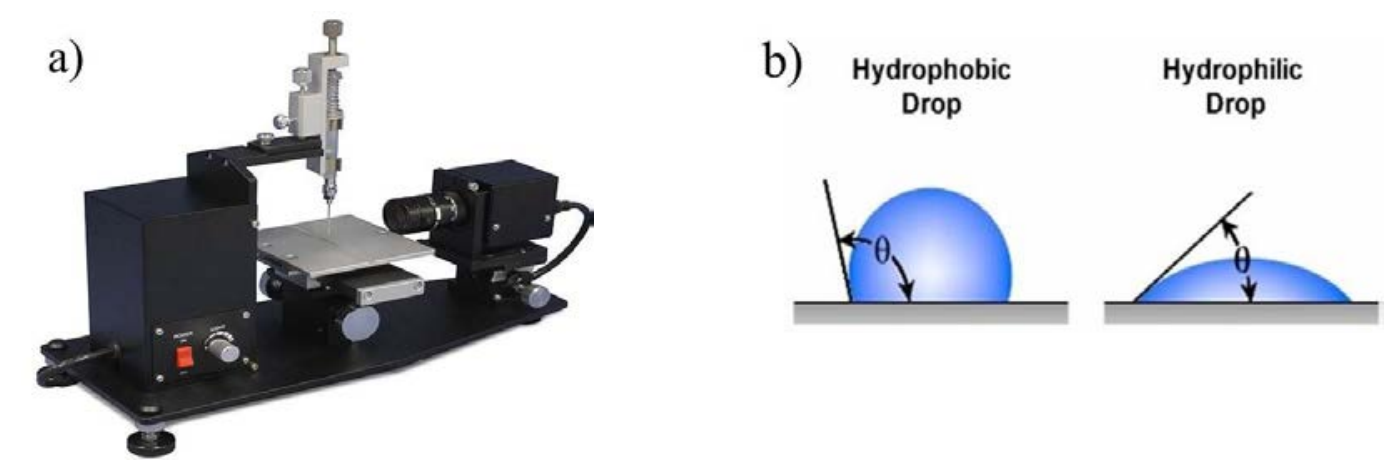

Figure 4.2: (a) Kyowa Contact angle meter and (b) Wetting angle made by a liquid over a solid substrate.

of the dropped image was determined for its contact angle $\left(^{\circ}\right)$ (Figure 4.2b) and surface free energy parameters of the sample were evaluated. From a scientific perspective, the understanding of liquid solid interactions is essential to understand adhesion, as this represents an ideal case of adhesive 
corrugating on a solid composite surface while curing. Duncan et al. [1] investigated solid-liquid interactions of adherends and adhesives and found that an adhesive will wet the adherend when its surface energy is lower than the adherend. According to Young's Dupre equation for wetting angles greater than zero, the force balance at a solid-liquid boundary is given as

$$
\gamma_{\mathrm{lv}} \cos \theta=\gamma_{\mathrm{sv}}-\gamma_{\mathrm{sl}}
$$

where $\theta$ is the contact angle, and $\gamma_{s v}$ is the surface energy of the solid-vapor, $\gamma_{s l}$ is the surface energy of solid-liquid interface and $\gamma_{l v}$ is the surface energy of liquid-vapor interface. As the adhesive tends to wet the adherend, lower contact angles are obtained.

In this chapter, contact angles are first determined for baseline or pristine samples. These angles are then compared to contact angles measured from the contaminated specimens. Differences in these angles will then be correlated with bond strengths from both specimen types.

\subsubsection{FTIR Measurements}

Infrared spectra were collected using the JASCO 4100 series bench-top infrared spectrometer using the ATR-PRO450-S accessory. The ATR measures the change in the internally reflected infrared beam when the beam comes in contact with the sample. The changes in the energy due to absorption of the incident beam is detected which provides information about available functional groups on the sample surface. Information related to the available functional groups helps to better understand and identify the surface chemistry associated with the adhesive and can provide insights on its behavior. Care was taken to ensure full and intimate contact between the composite surface and the ATR germanium crystal with the application of moderate pressure over the surface as shown in Figure 4.3. A few FTIR systems purge Argon gas into the compartment to eliminate moisture and $\mathrm{CO}_{2}$. The JASCO 4100 series utilized does not possess this capability and hence, moisture and $\mathrm{CO}_{2}$ peaks were subtracted from the resultant spectra. The FTIR spectrum of the material can be compared for "best matches" with libraries of spectra that have been cataloged 
for known materials. The spectra were collected in $600 \mathrm{~cm}^{-1}$ to $1800 \mathrm{~cm}^{-1}$ region with $2 \mathrm{~cm}^{-1}$ resolution, 128 scans with scanning speed of $2 \mathrm{~mm} / \mathrm{sec}$.

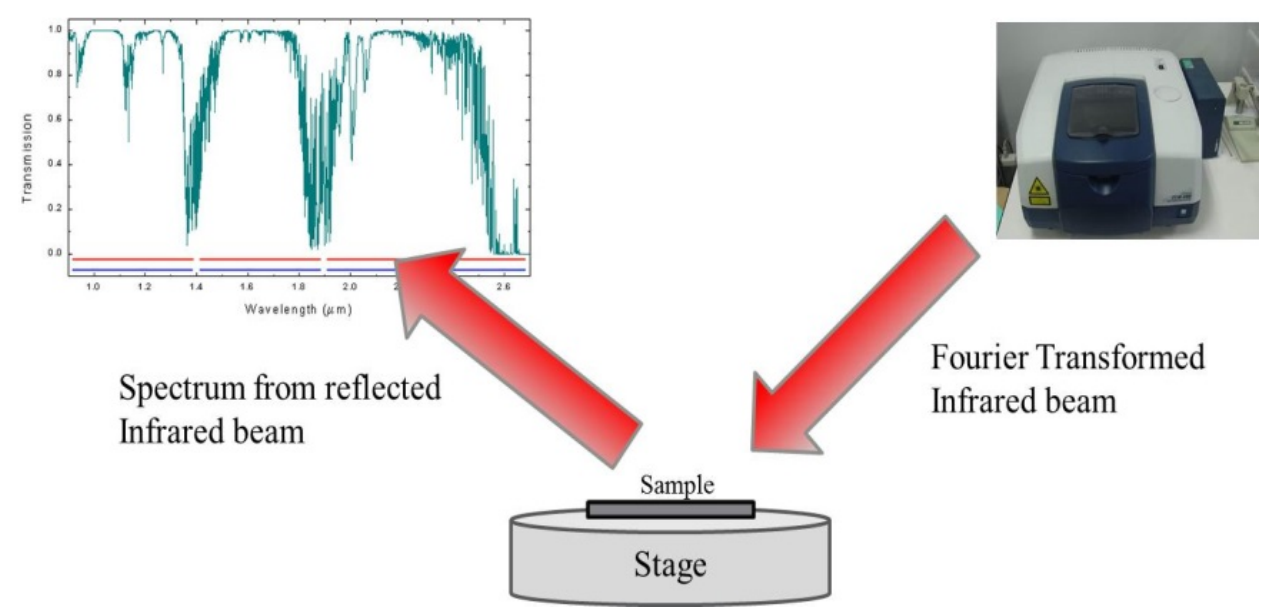

Figure 4.3: Schematic showing FTIR processing technique.

Samples from Toray prepreg and AF 555M adhesive were investigated before and after cure to identify the structural changes that occur in the material system. It should be noted that both the prepreg and adhesive are proprietary and very little information is known pertaining to their chemistry. The changes that are associated before and after cure can suggest the cure mechanism related to the materials and possibly assist in identifying dominant constituents of the material system. Prior to analysis, the peel ply was removed from the cured baseline samples and IR spectroscopy was conducted on the sample surface. IR spectroscopy was also conducted on the surfaces of the contaminated specimens and on the contaminant alone.

\subsubsection{Bondline Measurements}

Joint performance has been shown to be dependent on the bondline thickness and consistency of the bondline [2, 3]. When evaluating adhesive bond, bondline thickness measurement provide a means for assessing small variances in thickness and their effect on bond strength. The Optical Microscope utilized in this research was a petrographic Olympus BH2 and SZ9 scope with a camera Q-Imaging Micropublisher 3.3 RTV. The software used to analyze the 
acquired images was Q-Capture Pro. To provide a consistent bondline in our samples, a high finish aluminum plate was placed on top of the laminate to apply even pressure throughout the laminate. After secondary cure, the bondline thickness was evaluated using an Optical Microscope to determine the variability in the bondline. The measurements are read at $1 \mathrm{~cm}, 3 \mathrm{~cm}$ and $5 \mathrm{~cm}$ from the crack initiation point of the DCB coupons as shown in Figure 4.4. The red region is the precrack region and the black region is the bonded region of a DCB coupon. Bondline measurements help in validating the thickness of a bondline and determines the variability associated with the it.

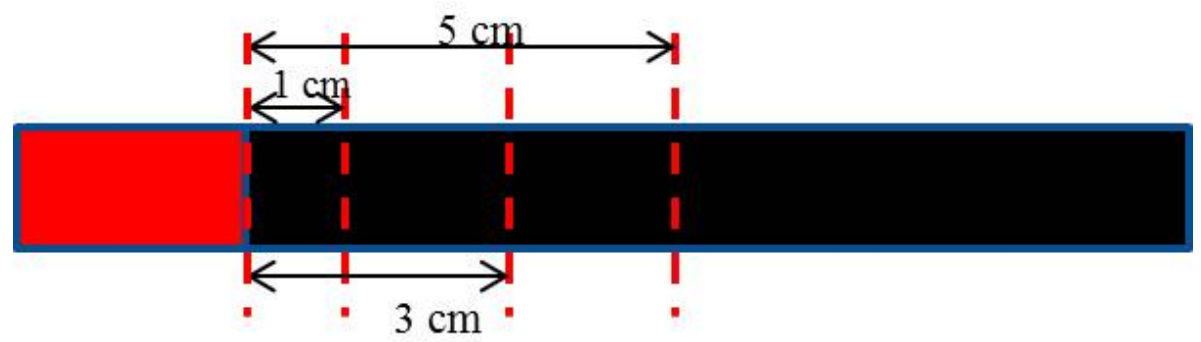

Figure 4.4: Schematic of the bondline measurement at $1 \mathrm{~cm}, 3 \mathrm{~cm}$ and $5 \mathrm{~cm}$.

\subsubsection{SEM/EDS microscopy}

Understanding the surface morphology of the composite laminate by itself as well as the adhesive to a nanometer resolution and their elemental composition will provide a significant information of the role of surface characteristics for adhesive bonding. A JEOL JSM 5900LV Scanning Electron Microscope (SEM) equipped with Energy Dispersive Spectrometer (EDS) was utilized to analyze the surface morphology and quantify the chemical composition of pristine and contaminated ABCJ's specimens. An accelerating voltage ranging from 15 to $30 \mathrm{kV}$ was used to investigate the surface characteristics which aided in distinguishing the elemental constituents or the chemical difference between the pristine and contaminated set. Specimens were sputter coated with gold for 2 minutes to avoid charging. Additionally, qualitative information on chosen elements and their distribution was analyzed using EDS Line scan methods. In line-scanning method, the electron probe moves linearly along the ROI on the sample. As the probe moves, the detector 
records the counts of the signal, which is an indicator of the number of X-ray quanta being detected or counted. The sample was ensured to be on a perfect horizontal stage to minimize different emergence angles arising of an uneven plane. To maximize the detector output, the line scan was set to long dwell times which aided in collecting a reasonable density of counts within the profile.

\subsubsection{Gravimetric Analysis}

Assessing the weight changes in the laminate before and after contamination is of key importance when quantifying the contamination. Since contamination impacts the bond strength, it is worthwhile to establish/evaluate a relationship between the weight gain and the bond strength associated to that processing. A Metler Toledo AB304-S weighing system was utilized to demonstrate differences in the weight gain before and after contamination.

\subsection{Results and Discussions}

\subsubsection{Wettability Measurement}

The contact angles were measured on all the specimens which included baseline (noncontaminated), mesh contaminated and stamp contaminated. Initially, the contamination used was the aerosol and as discussed in Chapter 3. However, subsequent analysis was conducted using the stamped approach with frekote. Contact angle measurements were made with each set and are discussed below.

The aerosol mesh contaminated specimens had slightly higher angle measurements than

the aerosol stamp contaminated specimens for water and ethylene glycol liquids. The variation of contact angle for mesh and stamp approach is shown in Figure 4.5. Results show that the contact angles are lower using the stamp approach when compared to mesh approach. The increase in the contact angle for the mesh approach specimens is attributed to poor wetting characteristics [4] 


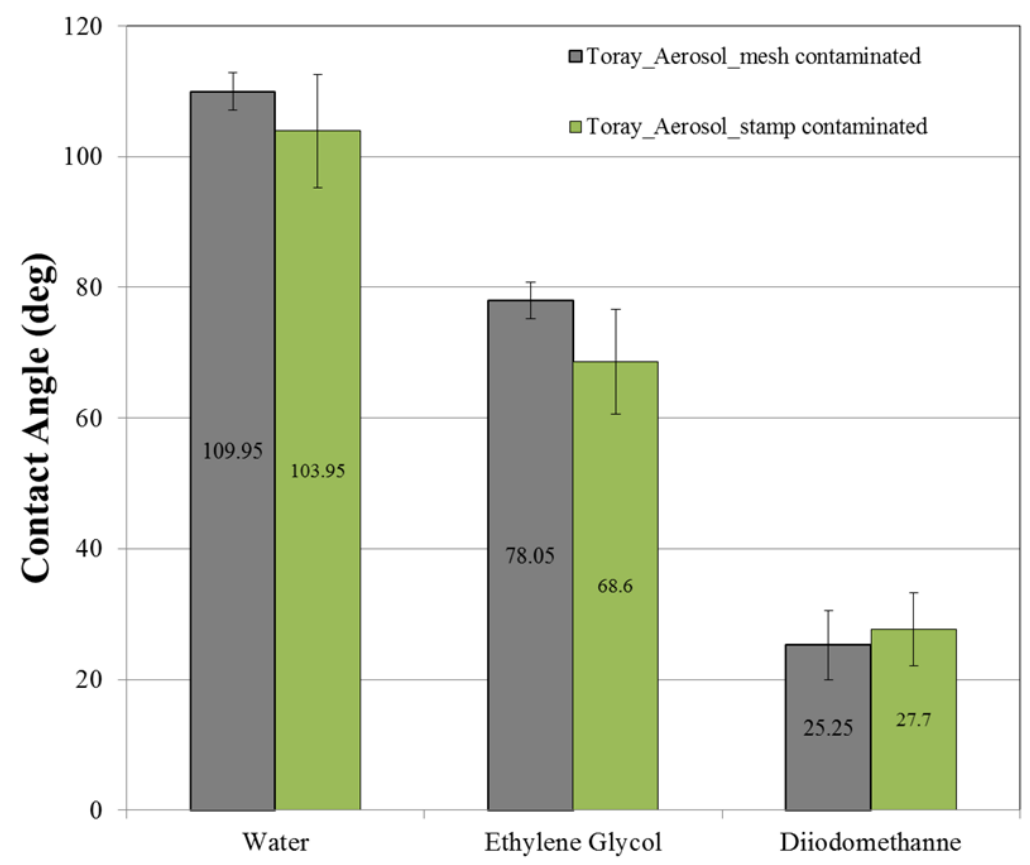

Figure 4.5: Contact Angle measurements for mesh and stamp approach using aerosol contaminant.

due to the inhomogeneity in the distribution of Aerosol through a mesh. Table 4.1 shows the wetting behavior of different fluids with the composite surfaces. Careful inspection reveals that the mesh contaminated specimens displayed a non-uniform spread or inhomogenous spread on the composite surfaces. On the other hand, when the contamination was distributed using the stamp approach a very consistent pattern and a homogenous distribution pattern was obtained. This is one of the many reasons in adopting the stamp approach largely due to its consistent patterning. Figure 4.6 shows a comparison between the non-contaminated (baseline) specimens to contaminated specimens i.e. aerosol (mesh and stamp) and Frekote contaminant. Lower angles were found for the baseline specimens as they are pristine and free of contaminants exhibiting a hydrophilic nature. Lower contact angle surfaces have better wetting characteristics, so that the adhesive wets out completely on a composite surface and mechanically interlocks itself on a 
Table 4.1: Wetting Angles with DI Water, Ethylene Glycol and Diiodomethane for Aerosol mesh and stamp contaminated specimens.

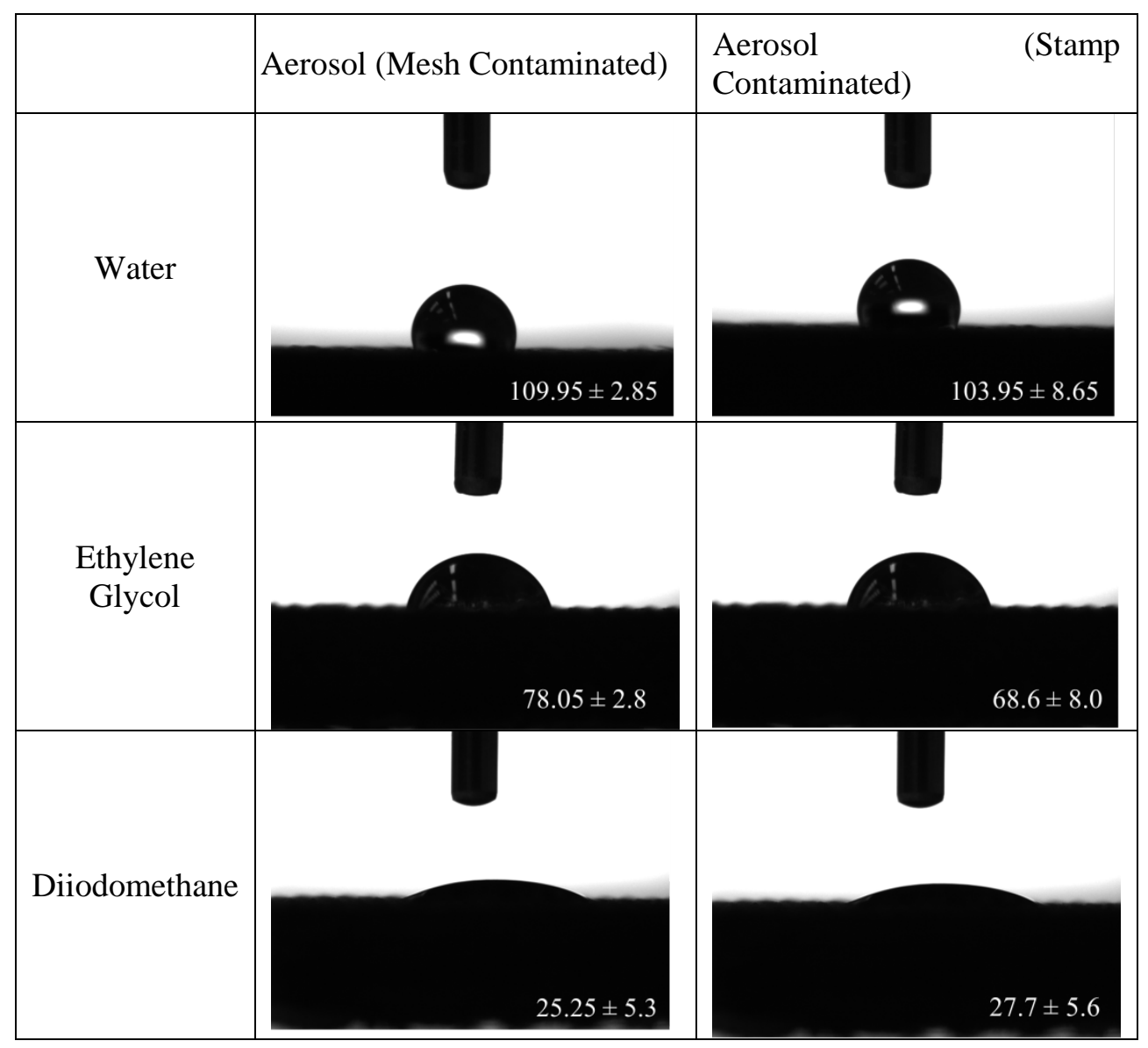

composite surface resulting in a better wettability. This mechanism can be correlated with the bond strength as pristine specimens have lower contact angles and the can possess greater strengths when compared with contaminated specimens. Table 4.2 shows their respective wetting angles. 


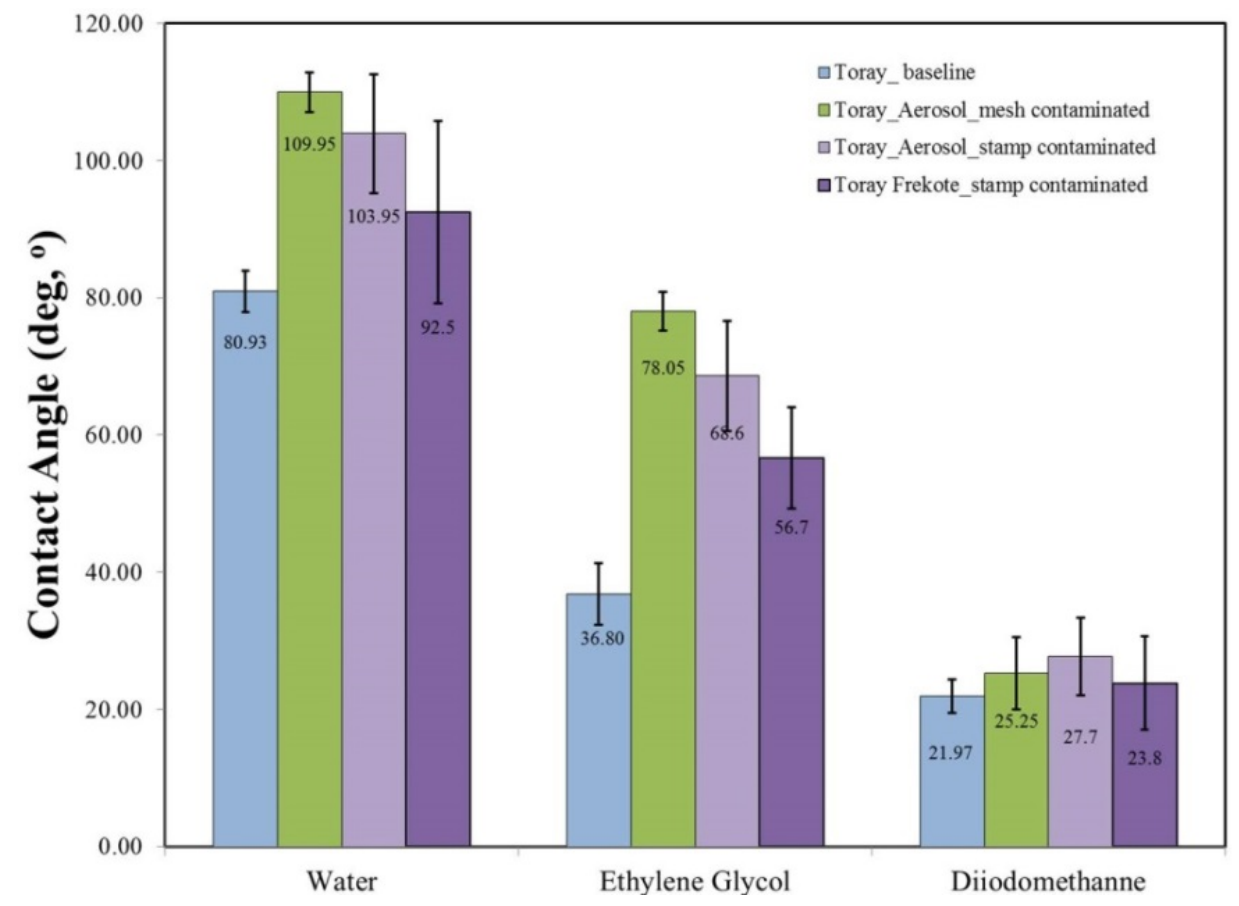

Figure 4.6: Contact angle measurements for baseline, aerosol (mesh and stamp) and Frekote specimens.

\subsubsection{Surface Energy measurements}

Surface free energy components have been evaluated using the dispersive, polar and hydrogen components of the energy balance equation. Under careful observation, the hydrogen component of the pristine specimen has a value of $3.3 \mathrm{~mJ} / \mathrm{m}^{2}$ while the toray frekote stamped specimens had hydrogen component of value $0.1 \mathrm{~mJ} / \mathrm{m}^{2}$. It should be noted that few specimens that were fully contaminated with frekote and conducting energy measurements on them revealed the hydrogen bonding component to be zero. This suggest that the pristine specimens have available hydrogen bonds to share with the adhesive while the contaminated specimens have marginal or nothing to share as hydrogen component. Surface Free Energies (SFE) were also calculated based on the contact angles obtained for the specimens and can be found in Table 4.3. The total surface free energy (SFE) of the baseline specimens and the aerosol mesh contaminated specimens did not 
vary significantly and the deviation is large due to non-homogeneity of the contaminant across the composite surface.

Table 4.2: Wetting Angles with DI Water, Ethylene Glycol and Diiodomethane for Pristine and Frekote stamp contaminated specimens.

\begin{tabular}{|c|c|c|}
\hline & Toray baseline (non-contaminated) & Frekote (Stamp Contaminated) \\
\hline \multicolumn{3}{|l|}{ Water } \\
\hline & $80.93 \pm 3.03$ & $92.5 \pm 13.3$ \\
\hline \multicolumn{3}{|l|}{ Ethylene Glycol } \\
\hline & $36.80 \pm 4.50$ & $56.7 \pm 7.4$ \\
\hline \multicolumn{3}{|l|}{ Diiodomethane } \\
\hline & $21.97 \pm 2.43$ & $23.8 \pm 6.8$ \\
\hline
\end{tabular}

However, the aerosol stamp controlled specimen did exhibit a large reduction in SFE when compared to frekote stamp controlled specimen. In general, a surface with low surface energy has poor adhesiveness i.e. large contact angles. This data correlates well with the contact angles obtained when compared to baseline specimen. 
Table 4.3: Surface Free Energy components of pristine and contaminated specimens

\begin{tabular}{|c|c|}
\hline Sample & Surface Free Energy, mJ/m2 \\
\hline Toray_Baseline (non-contaminated) & $51.0 \pm 4.5$ \\
\hline Toray_Aerosol mesh contaminated & $51.3 \pm 28.1$ \\
\hline Toray_Aerosol stamp contaminated & $36.2 \pm 10.9$ \\
\hline Toray_Frekote stamp contaminated & $46.2 \pm 3.9$ \\
\hline Toray_Frekote_all contaminated & $17.1 \pm 2.3$ \\
\hline
\end{tabular}

\subsubsection{FTIR Measurements}

FTIR Spectroscopic studies were conducted on the adherend and adhesive materials before and after cure i.e. as received and post cure. Since the materials are proprietary and company trade secrets are involved in it, it is difficult to identify exact constituents of the material but a close estimation can be made based on the IR spectra and literature. The changes that are associated with the spectra will reveal the cure mechanism.

\subsubsection{FTIR Spectroscopy on adherend}

The base polymeric compositions or the reactions involved in Toray prepreg system are first evaluated. Figure 4.9 shows the entire spectrum of the adherend (prepreg) material that is compared pre and post cure to identify the changes that occur during cure. Epoxy resin is one of the dominant constituent in a CFRP system and is identified as the characteristic peak occurring at $916 \mathrm{~cm}^{-1}$ for the before cure sample. The decrease of this band post cure reveals the consumption of epoxy group [5]. The peak disappearance in the cured composite is an indicative that the curing process is complete and has occurred fully. The curing mechanism generally involves amine addition to epoxy [6]. Epoxy crosslinks with amide of DICY involving in the ring opening of the epoxide is shown in Figure 4.7, forming a hydroxyl mechanism generally involves amine addition to epoxy [6]. Epoxy crosslinks with amide of DICY involving in the ring opening of the epoxide is 
shown in Figure 4.7, forming a hydroxyl and secondary amine. The epoxy concentration is decreased as the reaction gradually progresses which is seen in the IR spectrum in Figure 4.9 a.

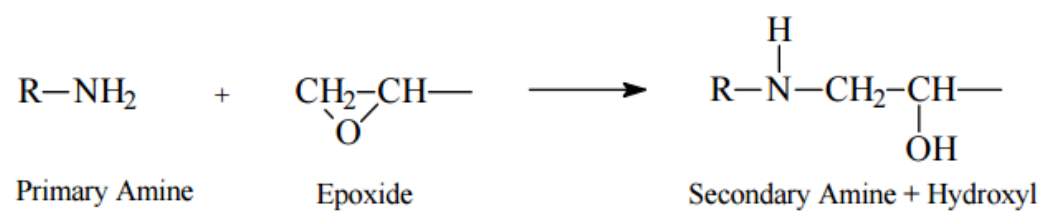

Figure 4.7: Primary Amine and Epoxy reaction

Conventionally, amine stretch (N-H) occurs with two strong bands between 3300 and 3500cm-1 while secondary produces only one band between 3350-3310 cm-1 while tertiary has no bands in this region [7]. After the attack of amine on epoxide group, the reaction progresses with the secondary amine and its hydroxyl group reacting with another epoxy group to form secondary hydroxyl group and a tertiary amine [8] as shown in Figure 4.8. This is clearly seen in FTIR spectrum where the before cure sample has two strong primary amine peaks between 3300-3500 $\mathrm{cm}^{-1}$ and are not visible in the after cure sample.

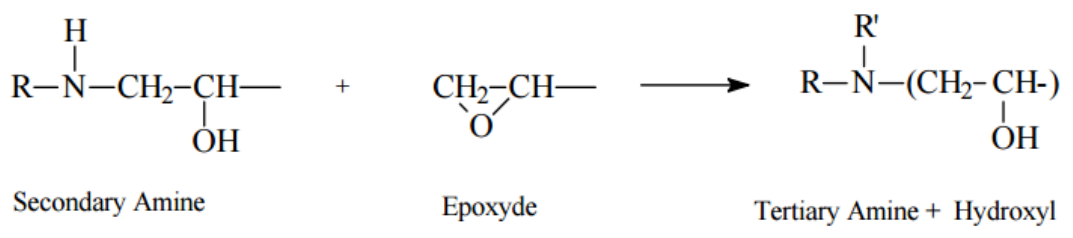

Figure 4.8: Secondary amine and hydroxyl reaction with epoxy.

Last, the etherification reaction follows [8, 9] between hydroxyl groups and remaining epoxide groups as shown in Figure 4.10. The leftover hydroxyl groups are seen in Figure 4.9 b) as a broad stretch in between $3500-4000 \mathrm{~cm}^{-1}$. The chain of reactions mentioned above gives us a clear understanding of the reaction mechanisms in an epoxy cured systems for high strength applications and serving as a good validation tool for the cure cycles. 

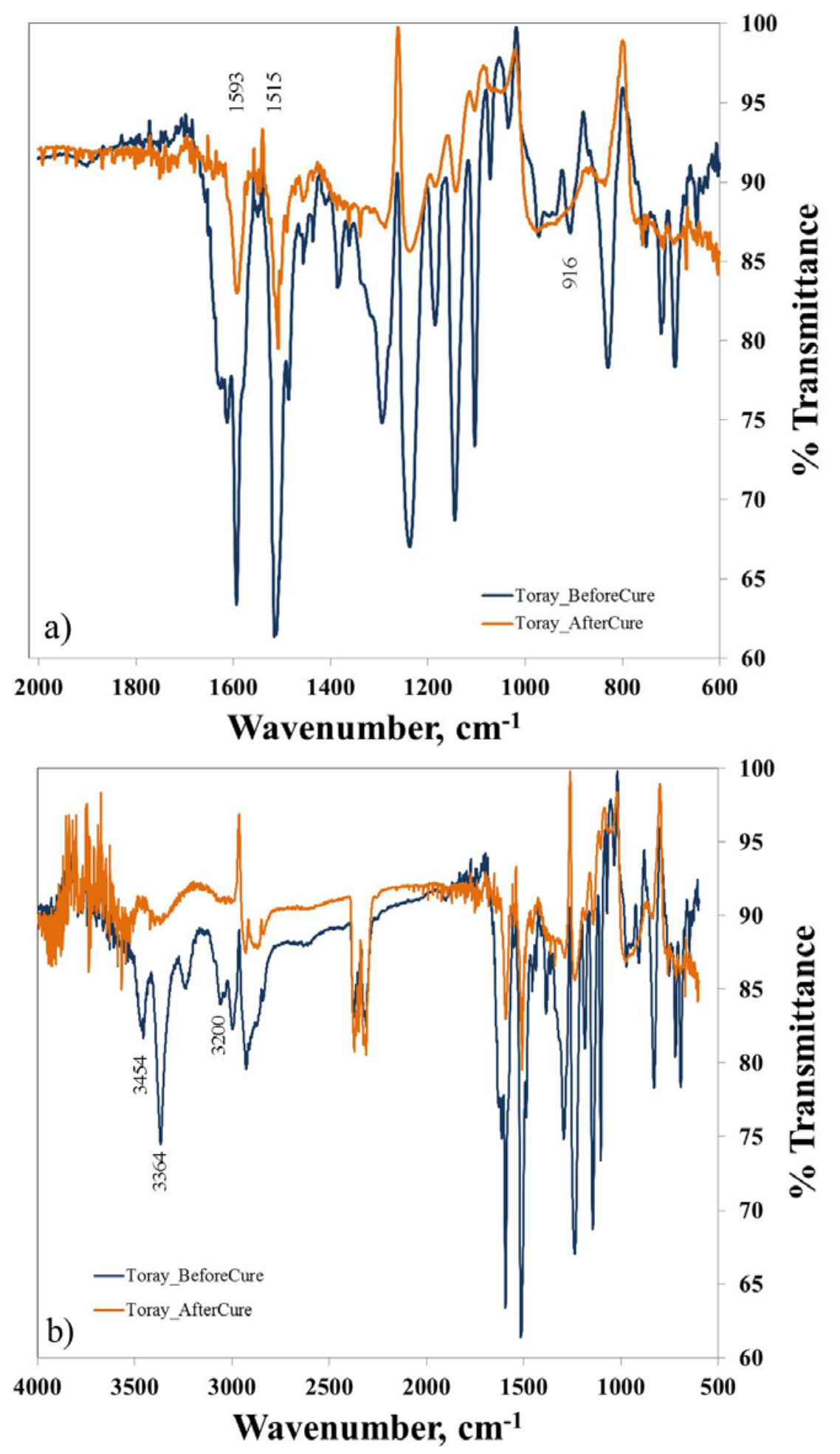

Figure 4.9: Comparison of FTIR Spectra of Toray adherend before and after cure a) Fingerprint region b) Full spectrum 


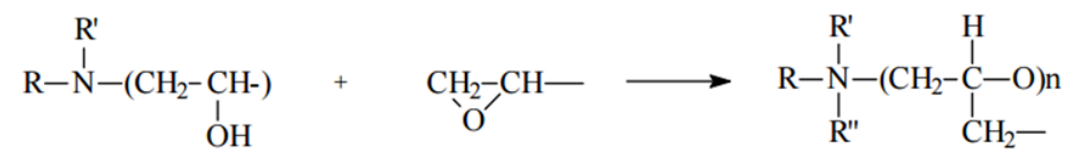

Tertiary Amine Epoxyde Q Quaternary Ammonium polyether

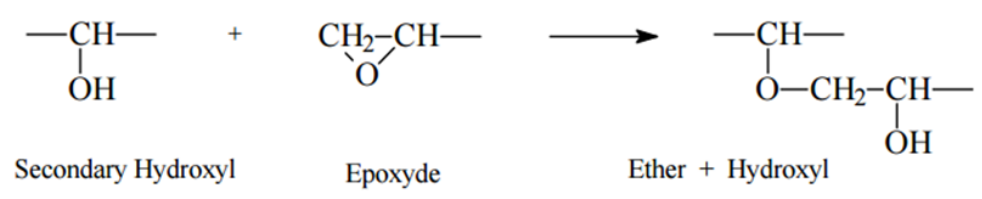

Figure 4.10: Tertiary Amine- Epoxy reactions followed by etherification

\subsubsection{FTIR spectroscopy on adhesive}

The base polymeric compositions or the reactions involved in AF 555M adhesive system are evaluated here. Figure 4.11a shows the entire spectrum of the adhesive material that is compared pre and post cure to understand the changes that occur during cure. The adhesive system by itself is an epoxy based adhesive possessing major constituent as epoxy and DICY as another curing agent which acts as a hardener. The curing of epoxy is very pronounce in adhesive at $916 \mathrm{~cm}^{-1}$ in before cure sample while has a decrease in its peak intensity in post cure sample. The characteristic doublet peaks between 1550- $1650 \mathrm{~cm}^{-1}$ are C-C stretches [7] where the hydroxyl groups of epoxy react totally and the peak disappearance is evident in post cure sample. At $2927 \mathrm{~cm}^{-1}$ a broad OH stretch band is visible in before cure sample while three small peaks at 300, 3038 and $3058 \mathrm{~cm}^{-1}$ are seen in post cure which represent C-H stretching of an epoxy group. This is associated with C$\mathrm{H}$ stretching of carboxylic acid as it usually exists as hydrogen bonded dimers [5]. Also a distinctive nitrile peak of DICY is seen at 2206 and $2161 \mathrm{~cm}-1$ which is the hardener and a curing agent used in the adhesive. This is consumed totally as the reaction proceeds which is validated by its absence in post cure samples. This dictates the strength of an adhesive. Uncured DICY's reactions can weaken the bond strength. Thus, FTIR assists in validating the cure mechanisms of uncured and cured adherends and adhesives. 

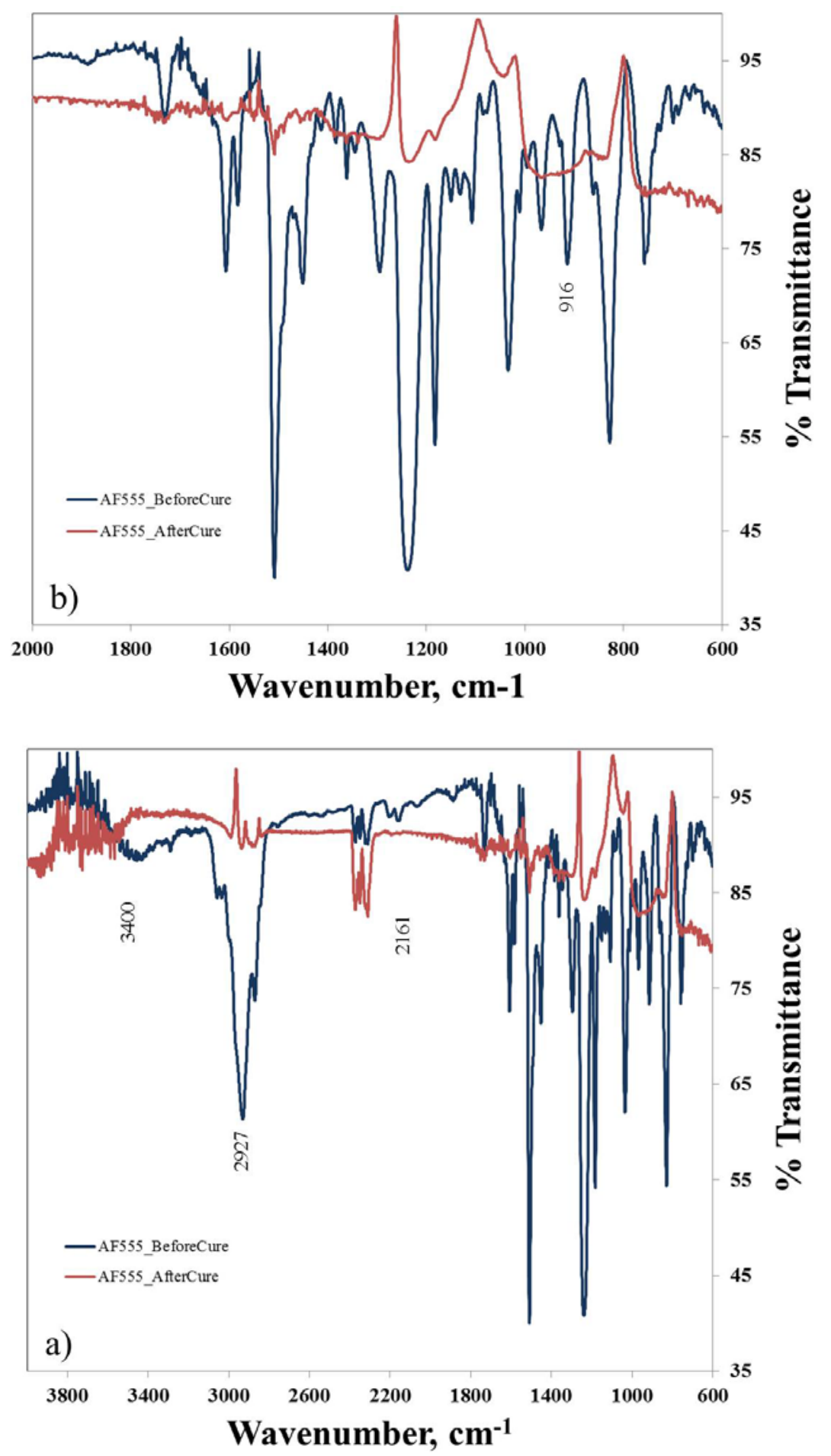

Figure 4.11: Comparison of FTIR Spectra of AF555M adhesive before and after cure a) Fingerprint region b) Full spectrum. 


\subsubsection{FTIR spectroscopy on contaminated specimens}

FTIR spectra of pristine composite laminates, contaminated laminates and contaminant alone were investigated for their surface chemical changes as shown in Figure 4.12. The frequencies and assignments of FTIR absorption bands are summarized in the Table 4.4. The broad stretching bands in the range $3364 \mathrm{~cm}^{-1}$ and $3454 \mathrm{~cm}^{-1}$ present in pristine and contaminated laminates correspond to O-H (Hydroxyl) group, and $1109 \mathrm{~cm}^{-1}$ is the characteristic peak for the primary amine $(\mathrm{N}-\mathrm{H})$ group. The presence of the $\mathrm{OH}$ group and $\mathrm{NH}$ group is indicative of the completion of the epoxy cure reaction during manufacturing as explained in section 4.2.2.1. It should also be noted that this identification of peaks validates the crosslinking process of epoxy and the curing process. A very intense band of Frekote contaminant in the range of $2928 \mathrm{~cm}^{-1}$ and $2856 \mathrm{~cm}^{-1}$ was observed that corresponds to $\mathrm{CH}_{2}$ and $\mathrm{CH}_{3}$ stretching vibrations $[10,11]$ which are associated with Polydimethyl-siloxane (PDMS) network [12], a major constituent of Frekote.

Table 4.4: Assignment of the Infrared absorption of epoxy resin with and without contaminant.

\begin{tabular}{|c|c|}
\hline Band (cm-1) & Assignment \\
\hline 3364,3454 & O-H stretch \\
\hline 2928 & CH3 symmetrical bend of Frekote \\
\hline 2856 & CH2 symmetrical bend of Frekote \\
\hline 1090 & Siloxane (Si-O-Si) \\
\hline $1020-1110$ & Siloxane (Si-O-Si) \\
\hline 864 & Methyl in Si-(CH3)3 \\
\hline
\end{tabular}

This is the strong intensity peak representative of Frekote i.e. methyl groups attached to Si. In

Figure 4.13 a, an expanded view of the IR spectra between wavenumbers 2800 and 3000 clearly shows the presence of strong broad bands of $\mathrm{CH}_{3}$ and $\mathrm{CH}_{2}$ in contaminated specimen revealing the 
cause for non-adhesive nature of the adhesive at the contaminated site due to their presence. Additionally, a detailed spectrum was further evaluated in the region 600 to $2000 \mathrm{~cm}^{-1}$, which is known as fingerprint region, to characterize the available functional groups. As shown in Figure $4.13 \mathrm{~b}$, the band corresponding to $1102 \mathrm{~cm}^{-1}$ has been identified as Si-O-Si in siloxane [11] [13, 10] in a PDMS cross linked network observed only in contaminated specimen. Peaks with identical similarities in both contaminated and non-contaminated specimens revealed that the contaminant frekote did not react with composite surface and no chemical changes on the surface were detected. The qualitative and quantitative similarity between the non-contaminated and contaminated specimen that are chemically identical, varied in their transmittance percentage signifying a very marginal or no change associated with the absorption of contaminant on composite surface.

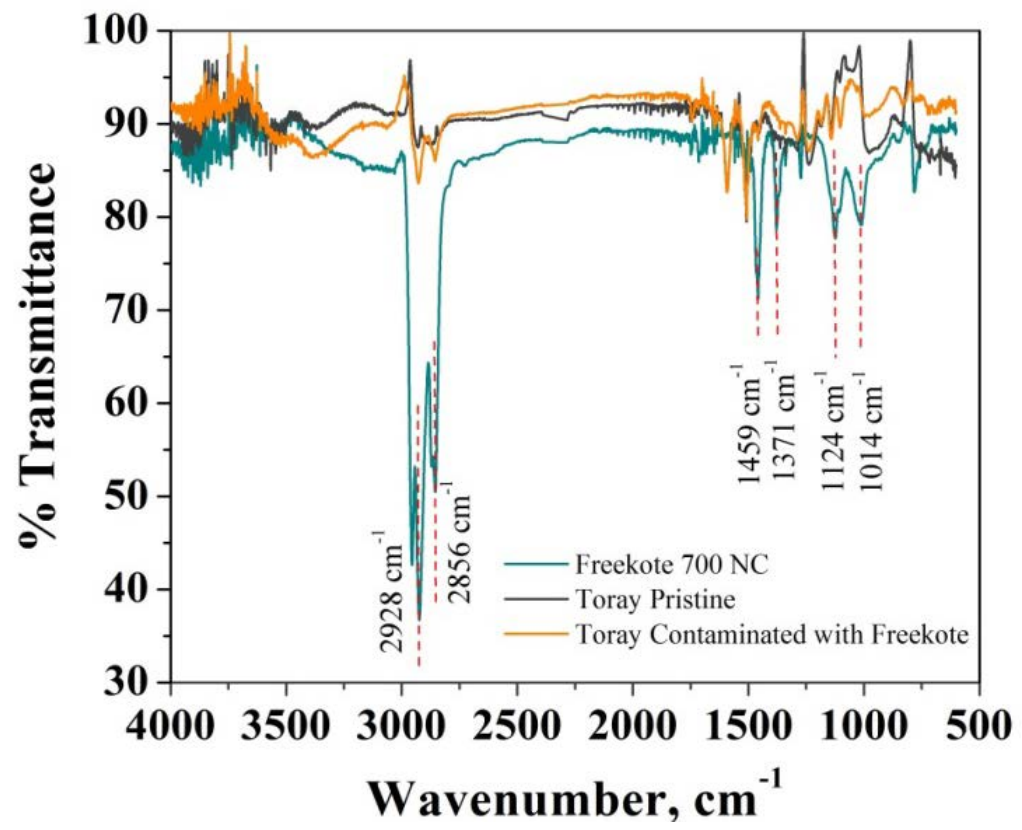

Figure 4.12: FTIR spectra comparing the Frekote contaminant, pristine composite surface and Frekote contaminated composite surface 

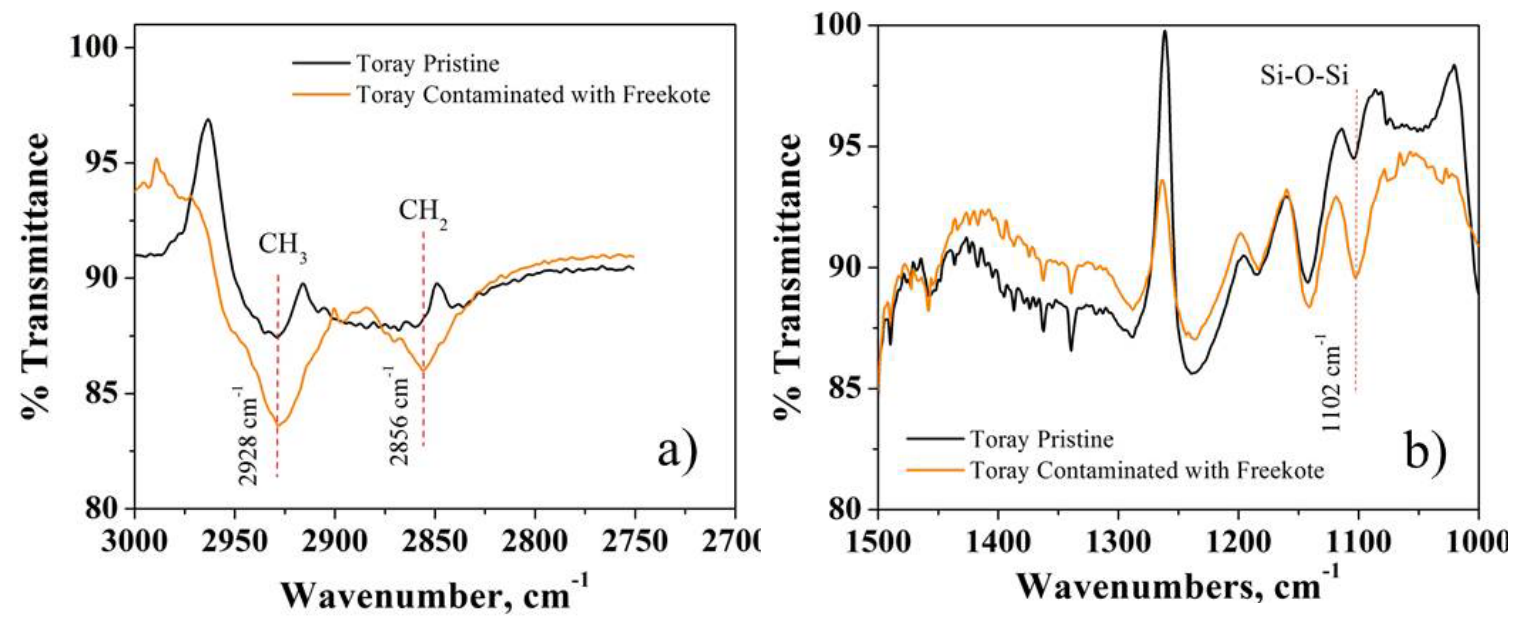

Figure 4.13: Expanded view of influencial bands in contaminated specimens in orange.

\subsubsection{Gravimetric analysis}

Normally when a polymer is subjected to any liquid, it tends to absorb the liquid due to its sorption characteristics. For the optimization of contamination route adopted in Chapter 3, the changes that are associated with their weights were recorded. This was also yet another reason to optimize stamp approach. The gain \% is shown in table and their respective charts below.

Table 4.5: Percent weight gain of laminate comparison between mesh and stamp approach

\begin{tabular}{|c|c|c|c|c|}
\hline \multicolumn{5}{|c|}{ Contaminant deposited-Aerosol } \\
\hline \multicolumn{2}{|c|}{} & Before (gms) & After (gms) & Gain \% \\
\hline $\begin{array}{c}\text { Aerosol Si } \\
\text { spray } \\
\text { (mesh) }\end{array}$ & Laminate 1 & 123.59 & 123.63 & 0.032 \\
\cline { 2 - 5 } & Laminate 2 & 122.52 & 122.53 & 0.008 \\
\hline $\begin{array}{c}\text { Aerosol Si } \\
\text { spray } \\
\text { (stamp) }\end{array}$ & Laminate 1 & 123.04 & 123.06 & 0.022 \\
\cline { 2 - 5 } & Laminate 2 & 124.18 & 124.21 & 0.024 \\
\hline
\end{tabular}

The distribution was non-uniform even after numerous trials via mesh approach as shown in Figure 3.11 with a significant variation in weight changes. The stamp approach showed promising results with a very low deviation in the gain percent and a uniform distribution. Stamp approach was 
henceforth adopted for future trails. The next task was to try and optimize other contaminants such as Frekote 710 -NC for further investigation. Figure 4.14 showcases Frekote contaminated specimen comparing aerosol and Frekote, both stamped.

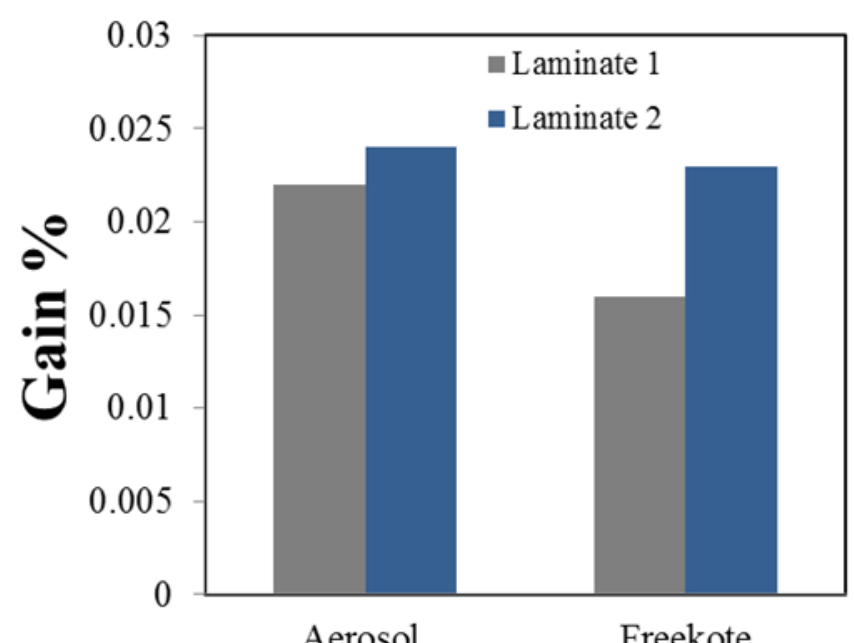

Figure 4.14: Weight gain comparisons between Aerosol and Frekote contaminant.

No significant change in weight gains were obtained between both the contaminants i.e. Aerosol and Frekote. Frekote resulted in creating weak bonds in comparison to Aerosol (see bond strength results), thus Frekote was adopted to be the ideal contaminant. Table 4.6 shows the weight gain \% for contamination done on single side (only one panel is contaminated while the other is left pristine) and dual side (two panels are contaminated as shown in Figure 3.12). No significant changes in weight have been identified in both single and dual contamination and are near uniform.

Table 4.6: Percent weight gain of contaminant on laminate between Single side and Dual side contamination.

\begin{tabular}{|c|c|c|c|c|}
\hline \multicolumn{7}{|c|}{ Contaminant deposited-Freekote } \\
\hline \multicolumn{2}{|c|}{} & Before (gms) & After (gms) & Gain \% \\
\hline $\begin{array}{c}\text { Freekote } \\
\text { 700NC }\end{array}$ & Laminate 1 & 121.35 & 121.37 & 0.016 \\
\hline
\end{tabular}




\begin{tabular}{|c|c|c|c|c|}
\hline (Dual side) & Laminate 2 & 125.46 & 125.49 & 0.023 \\
\hline $\begin{array}{c}\text { Freekote } \\
700 \text { NC } \\
\text { (Single side) }\end{array}$ & Laminate 1 & 122.41 & 122.44 & 0.024 \\
\cline { 2 - 5 } & Laminate 2 & NA & NA & NA \\
\hline
\end{tabular}

*Laminate 2 is designated as not applicable (NA) as only one side is contaminated

Moisture uptake specimens are being monitored to evaluate the net water ingress \% (absorption) into the specimen (bonded and non-bonded). The measurements are accurate up to four decimal points.

\subsubsection{Bondline Measurements}

Bondline measurements on the specimen post bonding have been determined for baseline specimen and contaminated specimen. 8 specimen for each set recording 18 data points on both sides of the coupons to evaluate an average bondline thickness. Baseline specimen possessed a 0.34 mm thick bondline while recorded a bondline of $0.26 \mathrm{~mm}$ and A3 recorded a bondline of $0.28 \mathrm{~mm}$. The values ranged between $0.26 \sim 0.34 \mathrm{~mm}$. Typical bondline for a specimen is showed in Figure 4.15. While the bondline thickness obtained for the adopted route in optimizing contaminant are as follows. Overall the bondline thickness varied between $0.23 \mathrm{~mm}$ to $0.34 \mathrm{~mm}$ for the manufacturing procedures adopted and the levels of optimization that was done on contaminant.
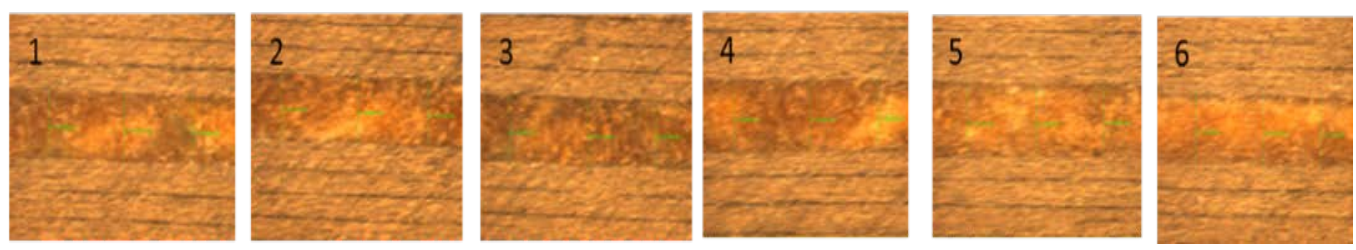

Figure 4.15: Bondline measurements conducted on a pristine specimen.

Table 4.7: Average bondline thickness $(\mathrm{mm})$ for all the test cases mentioned in section 3.4

\begin{tabular}{|c|c|}
\hline Specimens $(\mathbf{n}=\mathbf{4})$ & Bondline (mm) \\
\hline Toray_Aerosol (mesh) contaminated & 0.23 \\
\hline
\end{tabular}




\begin{tabular}{|c|c|}
\hline Toray_Aerosol (stamp) contaminated & 0.25 \\
\hline Toray_Freekote (stamp) contaminated-dual side & 0.32 \\
\hline Toray_Freekote (stamp) contaminated-single side & 0.33 \\
\hline
\end{tabular}

\subsubsection{SEM/EDS microscopy}

SEM/EDS analysis was conducted to investigate the contaminated interface and compare it to the non-contaminated interface to understand the influence of contaminant at the interface. Line scans across the interface were examined and care was taken that the contaminated region was sectioned exactly across the locus of contaminant region. This implies, at a microscopic level, near the loci of contaminant, there should exist a debonded interface. While the interface of a noncontaminated specimen or a region will be bonded cohesively to the adherend. Figure 4.16 shows a pristine interface of a non-contaminated specimen and no traces of debonding is seen whereas in Figure 4.17 which belongs to a contaminated specimen, a visible debonded area in white is seen at the interface.

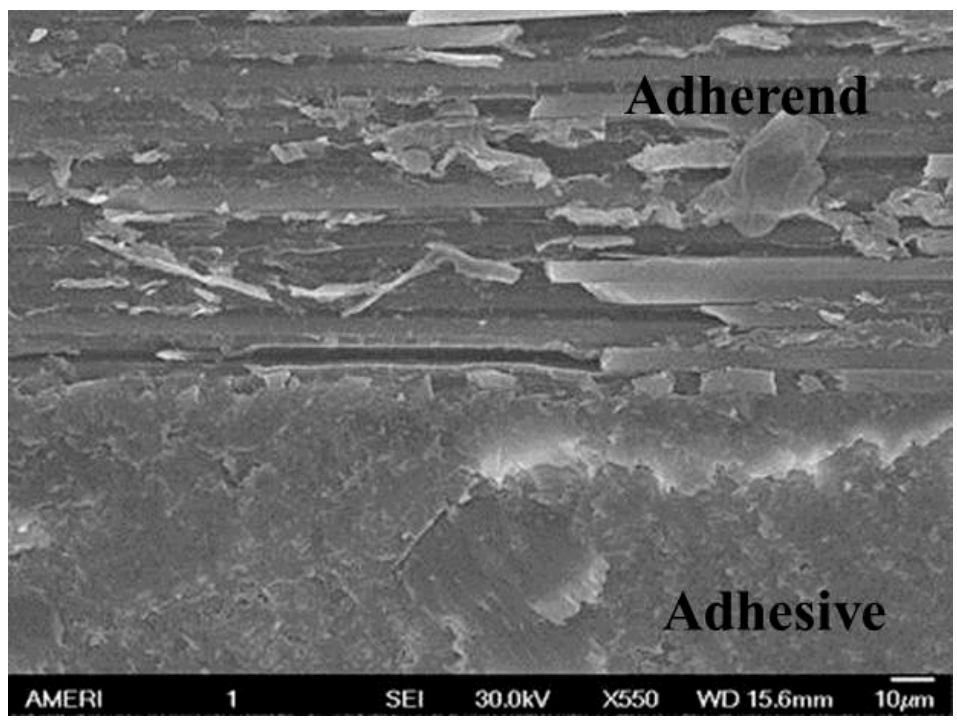

Figure 4.16: SEM image showing the interface of a non-contaminated specimen. 


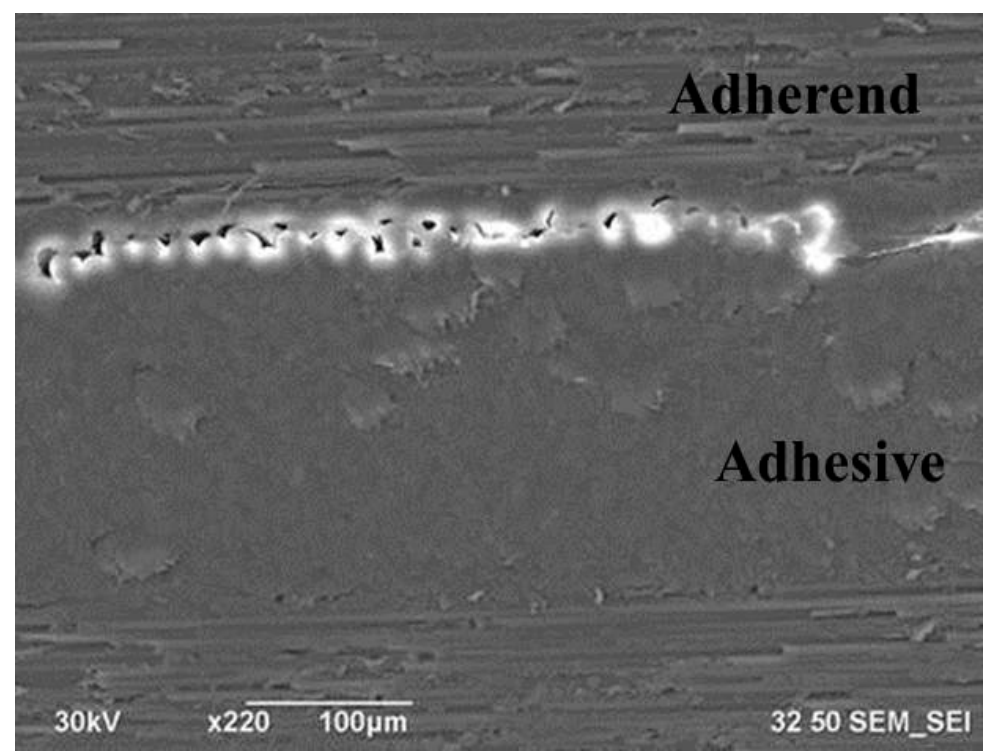

Figure 4.17: SEM image showing the interface of a contaminated specimen.

Line scans were performed in vertical directions on the same images to identify the chemistry of the contaminant as shown in Figure 4.18. Since the contaminant is dominant in PDMS, only Si element distribution across the line was scanned. In Figure 4.18b, a significant increase in Si content occurs at the debonded region where a large peak in counts occurred as the scan reached the crack region indicating the presence of Frekote at the interface. In Figure 4.18a, no Si elemental peak was observed in the region or at the interface. Distribution across the line was less than marginal and within noise due to longer dwell times.
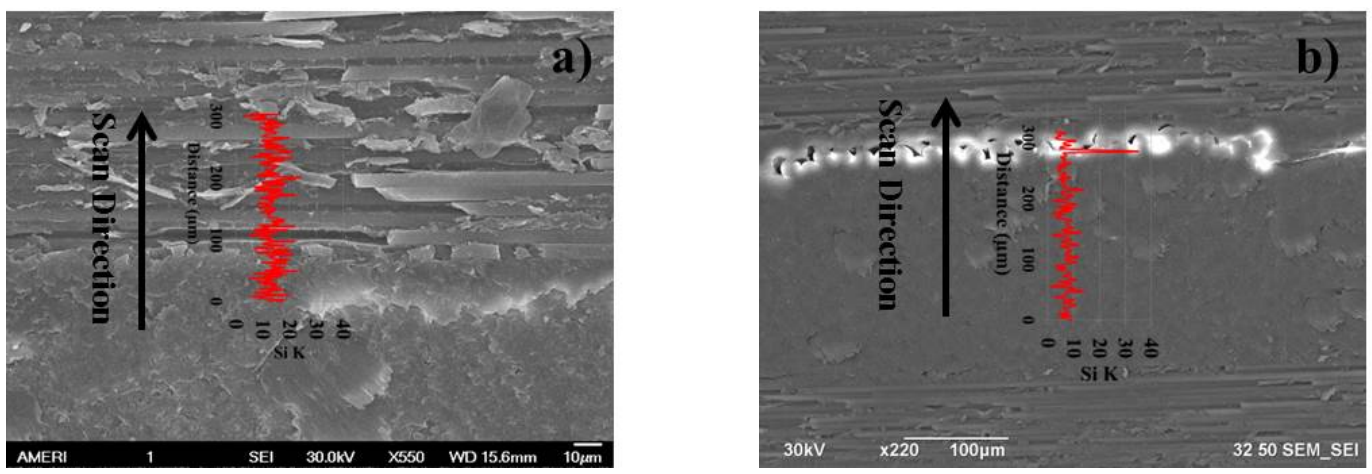

Figure 4.18: a) Elemental line scans of Si in the baseline/non-contaminated region and b) Elemental line scans of $\mathrm{Si}$ in the contaminated region across the interface. 


\subsection{Conclusions}

The results of this surface characterization study have been efficient in determining the surface response of an adherend or an adhesive in a bonded joint with superior performance. Wettability measurements were able to distinguish surfaces with contamination with the variation in the contact angle and their surface energy parameters that dictates the adhesive characteristics and predict its performance. FTIR measurements were able to discern the complex cure mechanisms by revealing the surface chemistry associated with adherend and adhesive. Additionally, a distinguishable difference in the FTIR peak intensities between pristine and contaminated specimens revealed the adhesion characteristics of a bonded joint in the presence of contamination such as Frekote. With the aid of gravimetric analysis, quantification of surface contamination was possible with the gain \%. For the manufacturing procedures adopted, bondline thickness varied between $0.23 \mathrm{~mm}$ to $0.34 \mathrm{~mm}$ which was consistent throughout. SEM/EDS line scans revealed the presence of siloxane at the interface in a contaminated specimen that can influence the strength of a bonded joint. The surface characterization tools utilized necessitates the need for characterizing surfaces of a composite material where the information obtained can be intrinsically linked to a composite structural performance. The contamination study can serve as a possible nondestructive evaluation (NDE) benchmark in predicting the adhesion characteristics involved with the bond quality prior to its implementation. For instance, hand-held FTIR scans can be used at various stages of composite processing/composite bonding to determine the presence of contaminate locations and overall contaminated area. 


\subsection{References}

[1] B. Duncan, R. Mera, D. Leatherdale, M. Taylor and R. Musgrove, "Techniques for characterising the wetting coating and spreading of adhesives on surfaces," National Physical Laboratory, Middlesex, UK, 2005.

[2] D. Gleich, M. Van Tooren and A. Beukers, "Analysis and evaluation of bondline thickness effects on failure load in adhesively bonded structures," Journal of Adhesion Science and Technology, vol. 15, no. 9, pp. 1091-1101, 2001.

[3] H. Qian and C. Sun, "Effect of Bondline Thickness on Mode-I Fracture in Adhesive Joints," in 49th AIAA/ASME/ASCE/AHS/ASC Structures. Structural Dynamics and Materials Conference, Schaumburg, IL, 7-10 April 2008.

[4] R. Dillingham and B. Oakley, "Surface Energy and Adhesion in Composite-Composite Adhesive Bonds," The Journal of Adhesion, vol. 82, pp. 407-426, 2006.

[5] C. Chihani, M. Mouzali and M. Abadie, "Study of Crosslinking Acid Copolymer/DGEBA Systems by FTIR," Journal of Applied Polymer Science, vol. 87, pp. 2033-2051, 2003.

[6] S. Chada, S. Adamala, M. Lu, W. Chian, C. Jenkins and R. Winter, "Combined FTIR/IFM Analysis of Bonded Joints," in SEM X International Congress \& Exposition on Experimental \& Applied Mechanics, Dearborn, MI, 2004.

[7] Organic Chemistry, University of Colorado, Boulder, "IR Spectroscopy Tutorial on Amines".

[8] G. Nikolic, S. Zlatkovic, M. Cakic, S. Cakic, C. Lancnjevac and Z. Rajic, "Fast Fourier Transform IR Chracterization of Epoxy GY Systems Crosslinked with Aliphatic and Cycloaliphatic EH Polyamine Adducts," Sensors, vol. 10, pp. 684-696, 2010.

[9] Q. Wang, B. Storm and L. Houmoller, "Study of the Isothermal Curing of an Epoxy Prepreg by Near Infrared Spectroscopy," Journal of Applied Polymer Science, vol. 87, no. 14, pp. 2295-2305, Jan 2003.

[10] G. Critchlow, R. Litchfield, I. Sutherland, D. Grandy and S. Wilson, "A review and comparative study of release coatings for optimised abhesion in resin transfer moulding applications," International Journal of Adhesion \& Adhesives, vol. 26, pp. 577-599, 2006.

[11] C. Jeenjitkaew, Z. Luklinska and F. Guild, "Morphology and surface chemistry of kssing bonds in adhesive joints produced by surface contamination," International Journal of Adhesion and Adhesives, vol. 30, pp. 643-653, June 2010. 
[12] T. T. Velan and M. I. Bilal, "Aliphatic amine cured PDMS-epoxy interpenetrating network system for high performance engineering applications- Development and Characterization," Bulletin of Material Science, vol. 23, no. 5, pp. 425-429, 2000.

[13] J. Coates, "Interpretation of Infrared Spectra, A Practical Approach," Encyclopedia of Analytical Chemistry, 2000. 


\section{MECHANICAL STRENGTH CHARACTERIZATION}

This chapter explains the mechanical performance of the bonded joints for pristine (noncontaminated) and contaminated specimens using the stamp approach. The dependency of mechanical strength on the size of contaminated sites has been determined at multi-length scales i.e. micro scale and macro scales to better understand the crack propagations and damage initiation mechanisms in an adhesively bonded joint. Double Cantilever Beam (DCB) specimens were used to evaluate fracture toughness at the macro scale while End Notch Flexural (ENF) specimens were utilized to evaluate fracture toughness at the micro scale. Modes of failure were investigated via fractographic analysis and quantified using an Image Analysis technique which will be detailed in later sections of this chapter.

\subsection{Fracture Toughness Evaluation}

Evaluating fracture toughness based on fracture mechanics assists in efficient design practices and is largely determined by material properties such as resistance to crack, stress intensity factors, elastic modulus and testing parameters such as crack tip opening displacement (CTOD), loading rate etc. In other words, the materials response to mechanical deformation is determined in terms of fracture toughness, which can be evaluated based on the mode of loading and critical strain energy release rates that characterizes the mode. Table 5.1 provides standards and protocol comprehensively summarized by Zhang et al. [1] that evaluate various modes of testing bonded joints.

Table 5.1: Standards and Protocols for Mode I, ModeII and mixed-mode fracture.

\begin{tabular}{|c|c|c|c|c|}
\hline $\begin{array}{c}\text { Standards and } \\
\text { Protocols }\end{array}$ & Fracture & Specimen type & G Calculation method & Ref \\
\hline ASTM D3433-99 & Mode I & DCB, TDCB & SBT & {$[2]$} \\
\hline ASTM D5528-13 & Mode I & DCB & SBT, CBT, ECM & {$[3]$} \\
\hline ISO 15024:2000 (E) & Mode I & DCB & CBT, MCC & {$[4]$} \\
\hline
\end{tabular}




\begin{tabular}{|c|c|c|c|c|}
\hline ESIS TC4 Protocol & Mode II & ELS, NF & CBT, ECM, CCM & [5] \\
\hline
\end{tabular}

*TDCB- Tapered Double Cantilever Beam

*SBT-Simple Beam Theory

*CBT-Corrected Beam Theory

*ECM-Experimental Compliance Method

*MCC-Modified Compliance Calibration

*CCM- Compliance Calibration Method

Several investigations that utilized Mode I type fracture to evaluate fracture toughness either observe crack propagation visually or within $5 \%$ maximum compliance increase $[6,7,8]$. However the dynamics of recording crack propagation should be instantenous and it complicates the situation with the aforementioned technique. Hecker et al. [9] monitored the crack propagations using a camera assisted DCB technique where a crack is detected via an optical techniques. Mode II static and dynamic loading conditions were investigated by Mall et al. [10] while Martin et al. [11] investigated on laminated composites via a four point bend, end notch flexure test to evaluate fracture toughness. However, the current research has explored Mode II type of loading in bonded joint at a different length scale (micro-scale) to understand the deformation mechanism while following the ASTM D5528 for macro scale.

\subsubsection{Macro-Scale Testing}

DCB testing was conducted on an Ultimate Tensile Machine (UTM), an MTS 858 Table Top System manufactured by MTS. DCB tests are conducted to determine the interlaminar fracture toughness and provides information such as energy release rate, crack growth and load-material specific failure mode. Due to its simple configuration and loading in tension, Mode I methods of testing have received much attention and is widely accepted and recognized for bonded composite joints [12]. For this investigation, the deflection applied was at a rate of 2.5 to $5 \mathrm{~mm} / \mathrm{min}$. With the increments in the deflection/loading rate, the delamination length (crack length) was measured on one side of test specimen with the aid of a travelling microscope. The delamination onset was marked with the help of a silver marker starting from the end of crack insert and gradations were 
made for every $1 \mathrm{~mm}$ for the first $5 \mathrm{~mm}$ and continuing the vertical gradation for every $5 \mathrm{~mm}$ from thereon as shown in Figure 5.1. The DCB specimens measured $292.1 \mathrm{~mm}$ in length and $25.4 \mathrm{~mm}$ in width with a precrack of $63.5 \mathrm{~mm}$. The results of all the DCB tests are calculated as per ASTM D 5528 [3]. The calibration method adopted was Modified Beam Theory (MBT) method where the expression of strain energy release rate is given as follows:

$$
G_{I}=\frac{3 P \delta}{2 b a}
$$

where $\mathrm{P}$ is the load, $\delta$ is the load point displacement, $b$ is the specimen width and $a$ is the crack length. In practice, this will overestimate $\mathrm{G}_{I}$ as the beam rotation effects will take place due to bad clamping or non-symmetric glueing of piano hinges. Correcting for rotation will introduce a factor $\Delta$ letting us to consider a slightly longer crack length i.e. a $+|\Delta|$, where $\Delta$ is calculated experimentally by generating a least squares plot of the cube root of compliance, $C^{1 / 3}$, as a function of crack length. The compliance, $\mathrm{C}$, is given by

$$
C=\frac{\delta}{P}
$$

i.e. ratio of the load point displacement and applied load.
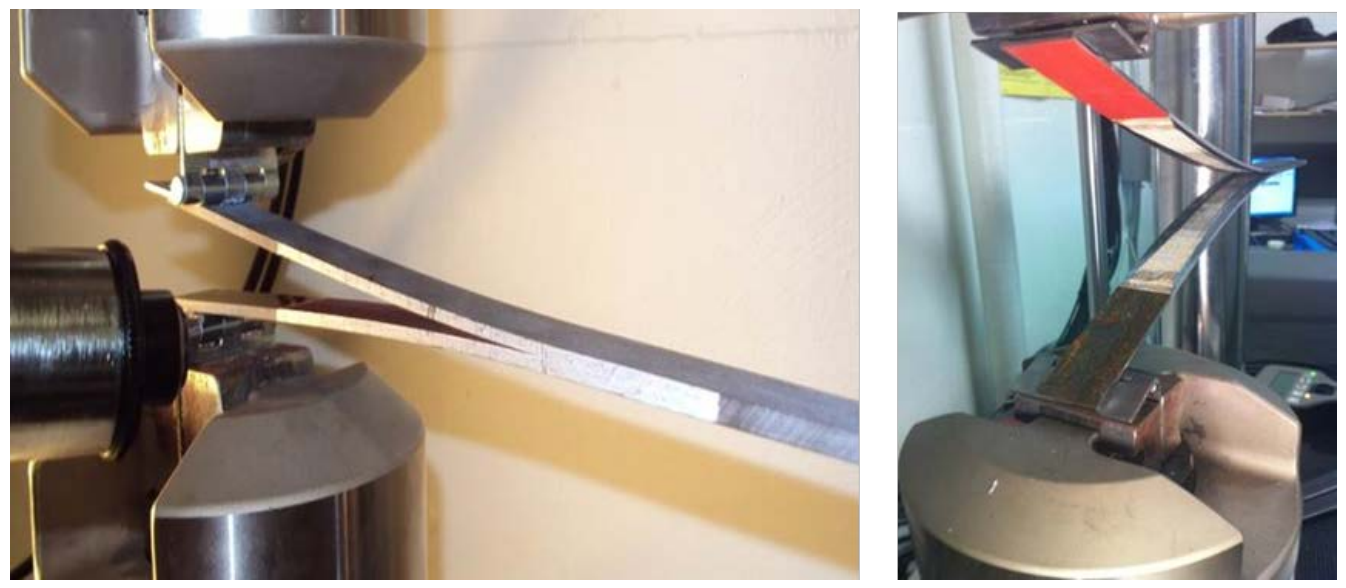

Figure 5.1: DCB Experimental setup with bonded specimen under load 
Compliance can be plotted as a function of crack length as shown in Figure 5.2. The values thus obtained correspond to visually observed crack propagation on the edges and serve as an input parameter to calculate energy release rate.

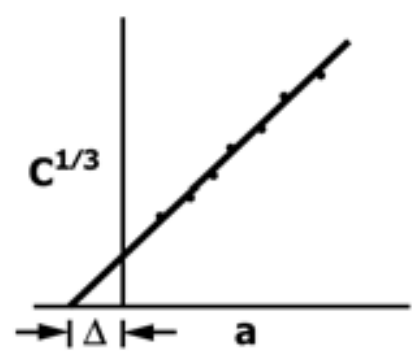

Figure 5.2: Complinace correction using Modified Beam Theory (MBT).

With the compliance correction factor, the energy release rate is:

$$
G_{I}=\frac{3 P \delta}{2 b(a+|\Delta|)}
$$

\subsubsection{Micro-Scale In-Situ Testing}

In practice, the micromechanical interaction of fiber and matrix at ply boundaries is a complex nonlinear problem, so efforts were made to understand the damage initiations in an adhesive layer at a micro scale on a bonded composite joint. A micro load frame (MTI Instruments SEM Tester 1000) mounted within the chamber of an electron microscope (JEOL JIB-4500 SEM/FIB) was utilized for micro-scale evaluation. A schematic of the setup is shown in Figure 5.3 a. Samples were cut from fabricated baseline and A3 contaminated panels using a low speed diamond saw measuring $40 \mathrm{~mm}$ in length and $10 \mathrm{~mm}$ in width with a precrack length of $10 \mathrm{~mm}$ in length. A comparison of the load bearing capacity of the baseline sample and A3 contaminated samples was conducted using a pre-cracked sample loaded in a four-point bend configuration. The pre-crack length and bondline thickness of both samples were measured using electron microscopy prior to testing. Testing was completed under load control at a rate of $200 \mathrm{~N} / \mathrm{min}$ with simultaneous imaging using the electron beam and the initiation of damage was monitored within the chamber 
in real time. SEM chamber pressure was maintained at $7.0 \times 10^{-4} \mathrm{~Pa}$ throughout testing. Samples were coated prior to testing with a thin Au layer to ensure conductivity.
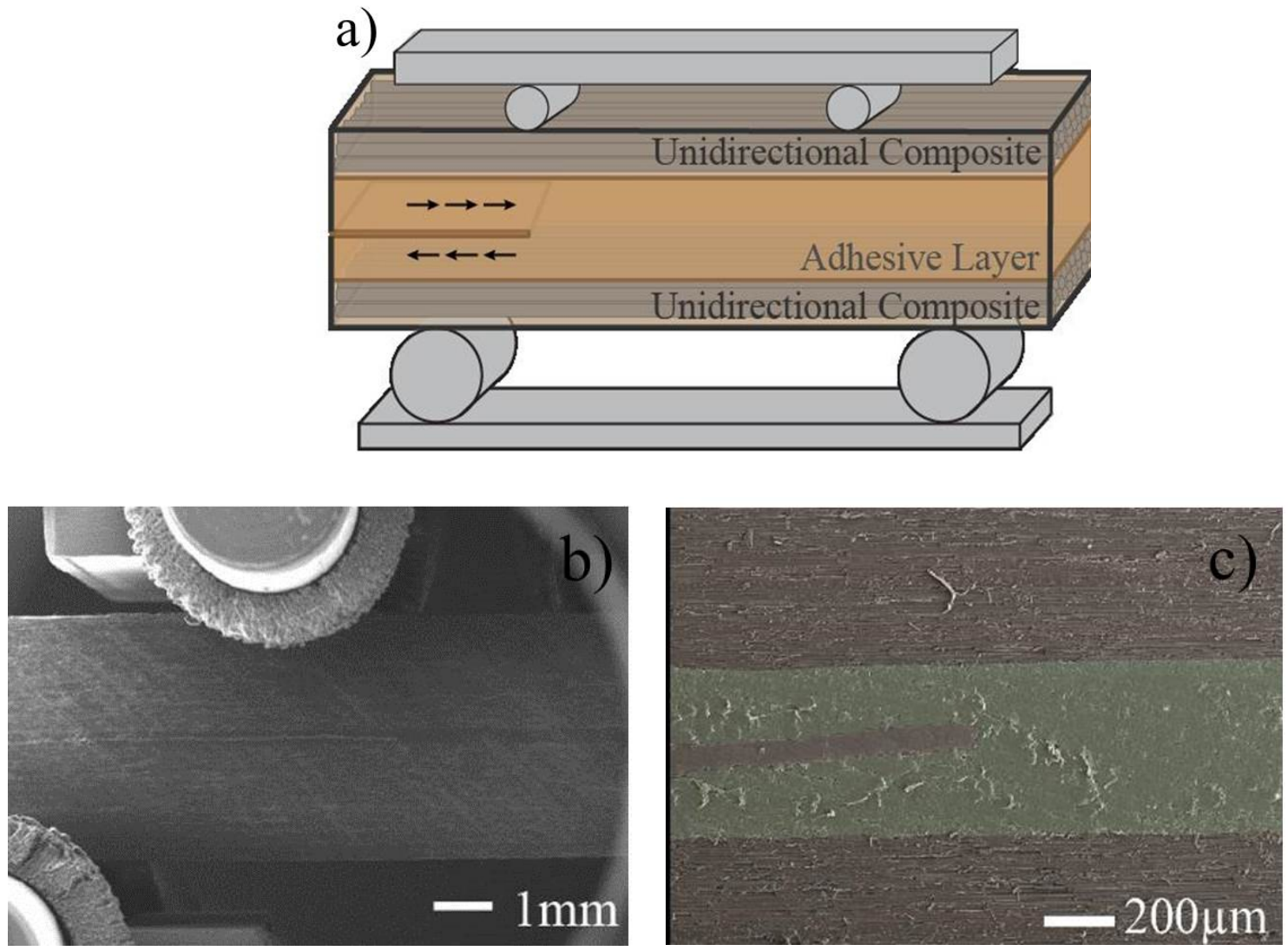

Figure 5.3 (a) Schematic of in-situ end notch fracture (ENF) test configuration (b) Low magnification SEM image of an ENF sample loaded in the 4 point bend fixture. (c) Higher magnification image of the crack tip region (false green coloring was added to delineate the region of adhesive.

\subsection{Quantification of Failure Modes}

Adhesive bonded joints can demonstrate considerable variation in their performance [13]. It is of prime importance to identify the key failures points that led to damage in a structural member [14], so that the failures can be understood and minimized in future. Further, the current state of understanding failures or failure modes in ABCJ's is limited to identification only, i.e. adhesive, cohesive or mixed mode failure, and not precisely quantify the modes. Attempts to establish a 
quantified relationship between the failure modes and bond performance have been emphasized in this research.

As mentioned in Chapter 2, contamination is an important but poorly understood component of ABCJ's. There is a derth of information on the effects of contamination on bond performance and its response to fracture. A low-cost methodology for quantification of failure mode geometry and its coverage area using image data captured via commercially available digital cameras is presented for the first time in bonded composites that could accurately quantify the failure modes and establish a relationship that can predict the bond strength that can be equally comparable to experimental datasets.

\subsubsection{Modeling Procedure/Digital Image Processing}

The tool utilized for image analysis quantification was a software known as ImageJ. ImageJ reads all graphic files such as JPEG, PNG, TIFF, BMP etc, and any specific region of interest (ROI) can be analyzed accurately. The advantage of ImageJ processing is its ease of evaluating ROI's and deselecting the unwanted areas. The software offers a unique pixel based architecture that can discern pixel ratios from digitized images, where each pixel within an image constitutes a single data point associated to certain brightness or color intensity. This translates to a color representation of the image or dataset captured. The digitally acquired image is processed through ImageJ software where a custom plugin is utilized to determine areas. The processed image then undergoes scaling/sizing to the relative pixel ratios. This ensures the length scale evaluations are appropriate and comparable. The user is then asked to identify the ROI followed by thresholding where the user iteratively adjusts the image pixels until a best map that fits the failure mode (ROI) is attained. The thresholded pixels are then visually enhanced to translate into the color maps that identify the areas of cohesive and adhesive failure. The relative area that is associated with the scale is thus quantified and the computed image is collated with the true mode of failures. 
Regions in which cohesive failure occurs are colored in red and the areas colored with black correspond to adhesive failure or interlaminar failure. Green areas are isolated marker components between the red and black areas and can be neglected for this analysis. Cohesive ratios and Interlaminar/Adhesive ratios are derived from the areas of each respective colored region as shown below:

$$
\begin{gathered}
\text { Cohesive ratio }=\frac{\text { Cohesive area of the specimen }}{\text { Total area of the specimen }} \\
\text { Interlaminar or Adhesive ratio }=\frac{\text { Non-Cohesive area of the specimen }}{\text { Total area of the specimen }}
\end{gathered}
$$

It should be noted that only the first $50 \mathrm{~mm}$ of damaged area starting from the precrack is considered for all the specimens according to ASTM D5528.

\subsection{Fractography Analysis}

A unique and advanced technique in optical microscopy was utilized to identify the crack propagation path called Line Profile analysis. In this method a line is drawn across the ROI and a profile is created based on the measurement of the macroscopic feature heights/depths. This aids in understanding the failure mechanism by revealing the topographic profile of the line showing the hils and valleys that represent the path of the crack. The failed surfaces from tested joints were analyzed to inspect the failure modes of both non-contaminated and contaminated specimens. The specimens were examined for any defects on the failure surface. For localized contaminated and non-contaminated site access, a line profile was rastered between two chosen points that was selected to cover both contaminated and non-contaminated sites measuring the surface profile of fractured adhesive. This aids in assessing the hills and valleys of the fractured adhesive.

\subsection{Results and Discussions}




\subsubsection{Micro-Scale Testing (ENF Testing/In-situ electron microscopy)}

The mechanism of failure and damage initiation were investigated for mode II propagation using ENF testing and in situ microscopy, with the resulting load-displacement data from in situ ENF testing shown in Figure 5.4. For the same loading conditions, the crosshead displacement was monitored and the final displacement is shown in the Figure 5.4. A representative baseline specimen and A3 contaminated specimen had a final displacement of $0.78 \mathrm{~mm}$ and $0.96 \mathrm{~mm}$, respectively. The increased bulk sample compliance ( 23\% increase after contamination) was likely a result of accretion of microscale damage in the specimen, with a reduction in the localized modulus due to microcracking as the sample was loaded. As seen in the macroscale DCB testing, application of the contaminate can simulate an occurrence of a weak bond or kissing bond, reducing the load bearing capacity within the region and ultimately stabilizing the crack opening leading to a reduced compliance. This hypothesis is confirmed in the concurrent microscopy, as shown in Figure 5.5. Prior to loading, both the baseline and contaminated samples show no signs of damage in regions directly in front of the crack tip. During loading, the damage evolution is significantly different in the baseline sample, as the damage is confined to the crack tip region with very marginal damage within the adhesive layer. During loading of the A3 contaminated sample, damage in the contaminated region initiates first and the crack begins to open and propagate (leading to the increased compliance seen in the load displacement curves). Then, very near to the crack tip region, a significant density of microcracks initiate and begin to coalesce. The most likely cause of these microcracks is the large stress concentration that initiates near the edges of the contaminated region. This is also attributed to the intermixing of Frekote contaminant with the adhesive during cure and settling at the localized regions where microcracks evolve. Damage in the baseline specimen was confined to the crack tip region while damage in the contaminated specimen initiated at the composite adhesive interface and propagated throughout the adhesive as loading increased. 


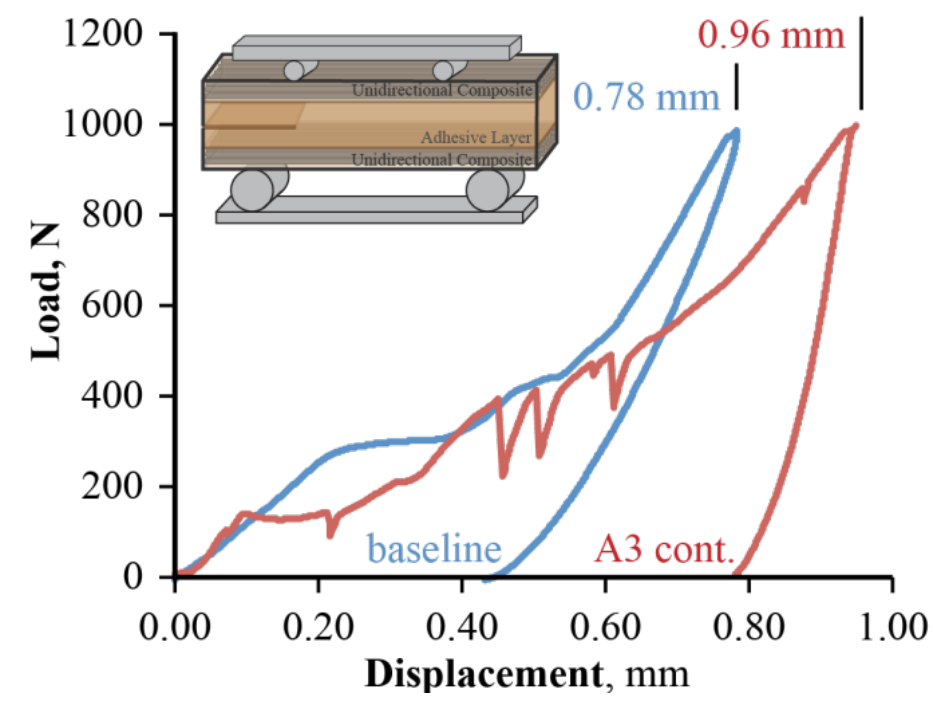

Figure 5.4. Load Displacement curves for baseline (non-contaminated) and A3 contaminated specimen using in situ ENF testing.

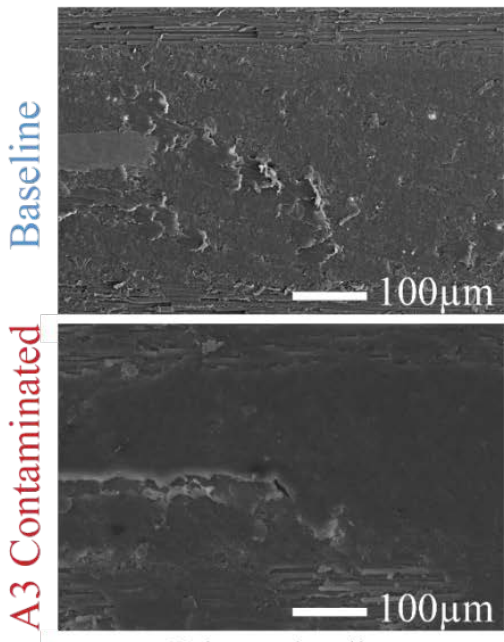

Prior to loading
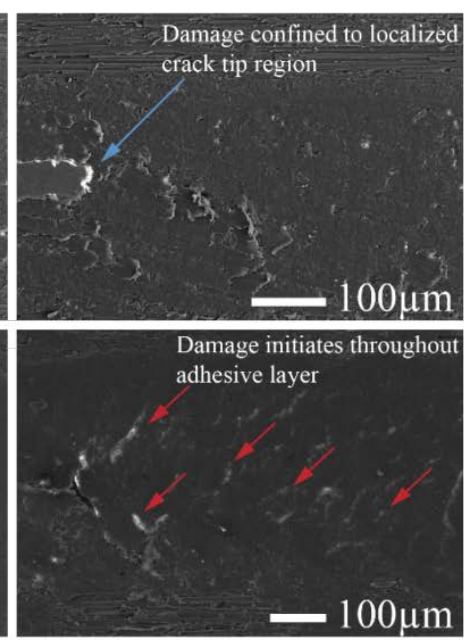

At peak load $(1000 \mathrm{~N})$

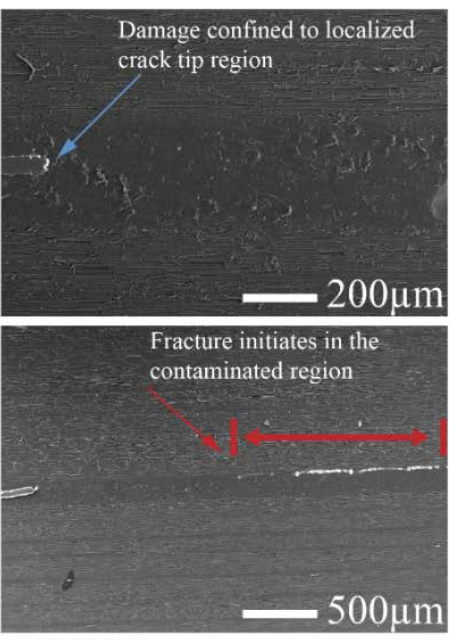
crack tip region

Fracture initiates in the contaminated region

Figure 5.5: Analysis of the damage initiation mechanisms for baseline and A3 contaminated specimens prior to loading and at peak load.

\subsubsection{Macro-Scale Testing (DCB Testing)}

The strain energy release rates associated with crack growth were calculated as per ASTM D5528 as mentioned in section 5.1.1. The $\mathrm{G}_{\mathrm{IC}}$ values between baseline aerosol (mesh and stamp) and Frekote are shown in Figure 5.6. The baseline bonded specimens (non-contaminated) exhibited an average $\mathrm{G}_{1 \mathrm{C}}$ of $0.74 \mathrm{~kJ} / \mathrm{m}^{2}$. The aerosol mesh contaminated set showed a marginal reduction at 
$0.65 \mathrm{~mJ} / \mathrm{m}^{2}$ and the stamp contaminated specimens exhibited an average value of $0.61 \mathrm{~kJ} / \mathrm{m}^{2}$. This indicates the contamination is weakening the interface causing reduction in the bond strength. The Frekote contaminated set exhibited a larger degree of reduction in the bond strength when compared to baseline specimens. The contaminated specimens suffered an average bond strength value of $0.45 \mathrm{~kJ} / \mathrm{m}^{2}$ to that of baseline specimens of $0.74 \mathrm{~kJ} / \mathrm{m}^{2}$. This ascertained the choice of contaminant in weakening the bond significantly.

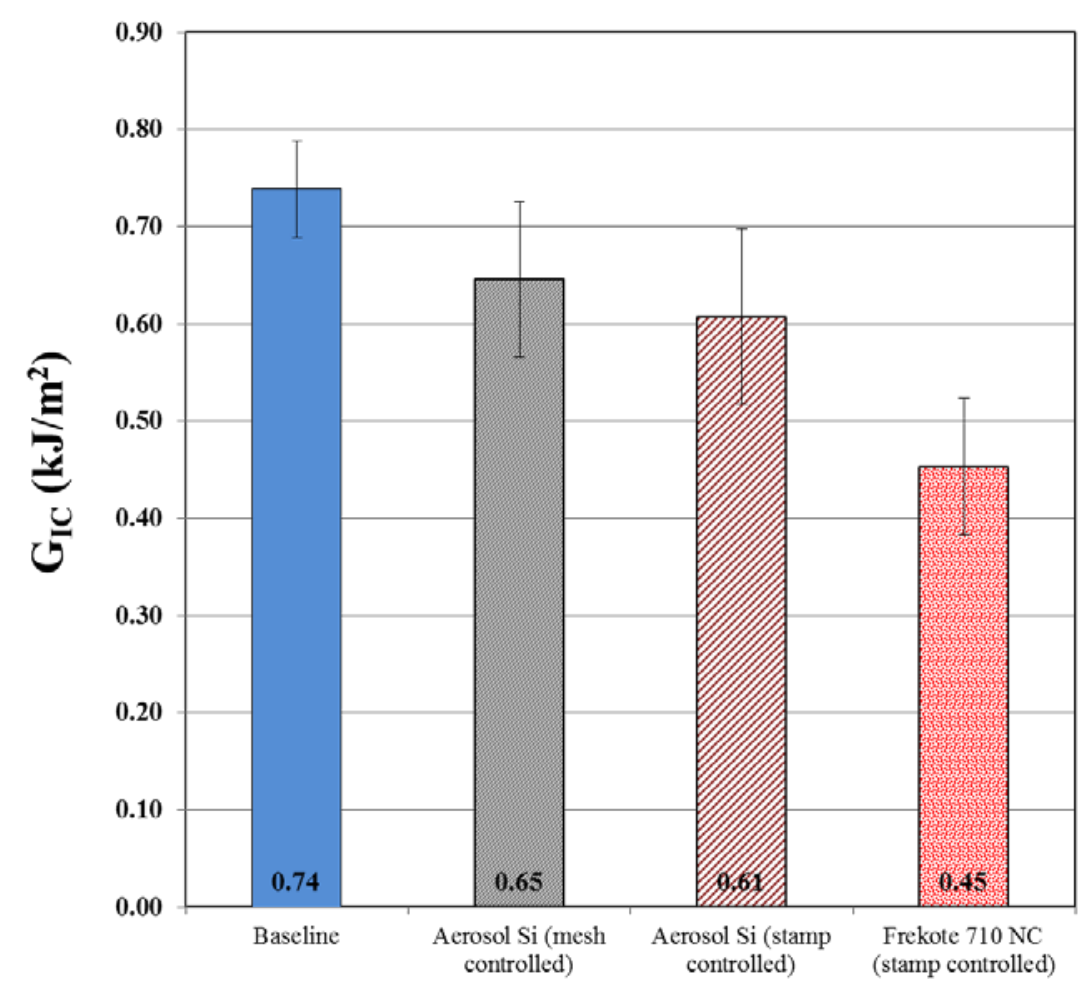

Figure 5.6: GIC values between baseline (non-contaminated), Aerosol (mesh and stamp) and Freekote (stamp).

The next steps was to investigate between the dual side contamination and the single side contamination and as mentioned in section 3.4.3. The results are shown in Figure 5.7 where single sided contaminated specimens (red dots on one side) of the interface showed similar reductions in bond strength when compared to the dual side contamination (red dots on both sides). Since the prime motivation was to drive the fracture towards the weakest link, single side contamination 
would help understand the crack propagation in an improved way than dual side contamination. Thus, single side contamination is adopted as the best approach for the remainder of this study.

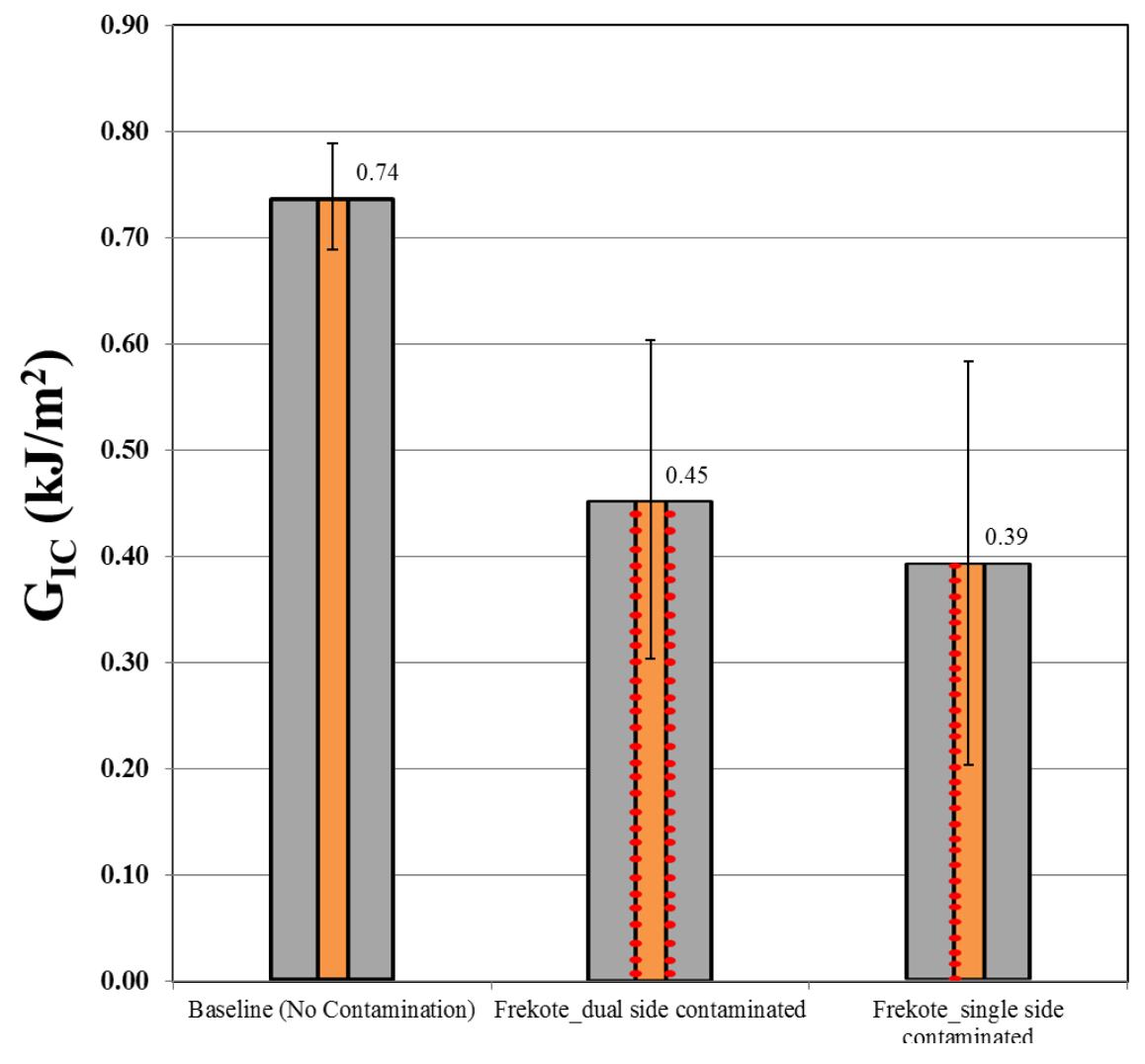

Figure 5.7: Comparison of $\mathrm{G}_{\mathrm{IC}}$ values between baseline (non-contaminated), dual side contamination and single side contamination with Frekote.

\subsubsection{Fracture Toughness Evaluations for Various Contamination Levels}

With the significant degradation results obtained for single side stamp contaminated specimens (see Figure 5.7), the contamination procedure was extended further by modifying the nature of stamp pattern i.e. increasing the level of contaminant as discussed in section 3.4.4. This will emulate UBC's criteria and provides substantial information on the influence of varying levels of contamination on fracture toughness. Using this approach, contaminant locations will create adhesion failure (failure at the composite-adhesive interface) that is intermixed with areas of cohesion failure (failure within the adhesive itself). By varying the size and application pressure of 
the stamp, an improved understanding of the influence of each type of failure on overall bond strength can be assessed.

Two stamps were used (see section 3.4.4) to investigate the effects of contaminated region size and total contaminated area on overall bond strength. To investigate the effects of contaminated region size, stamps A1 and A3 were used with the same application pressure (labeled as A1L and A3) and in this case the stamp was applied with only gravimetric force. Both stamps have equivalent starting coverage areas, therefore variations in bond strength can be attributed to contaminated region size (with variation in diameter of $1 \mathrm{~mm}$ vs. $3 \mathrm{~mm}$ ). As shown in Figure 5.8, it was found that the $\mathrm{G}_{1 \mathrm{C}}$ variability for $\mathrm{A} 3$ contaminated set was large. During the experimental runs, as the load increased, the instability of the crack in A3 specimens was high when compared to rest of the specimens. No fibre bridging was observed in any of the specimens.

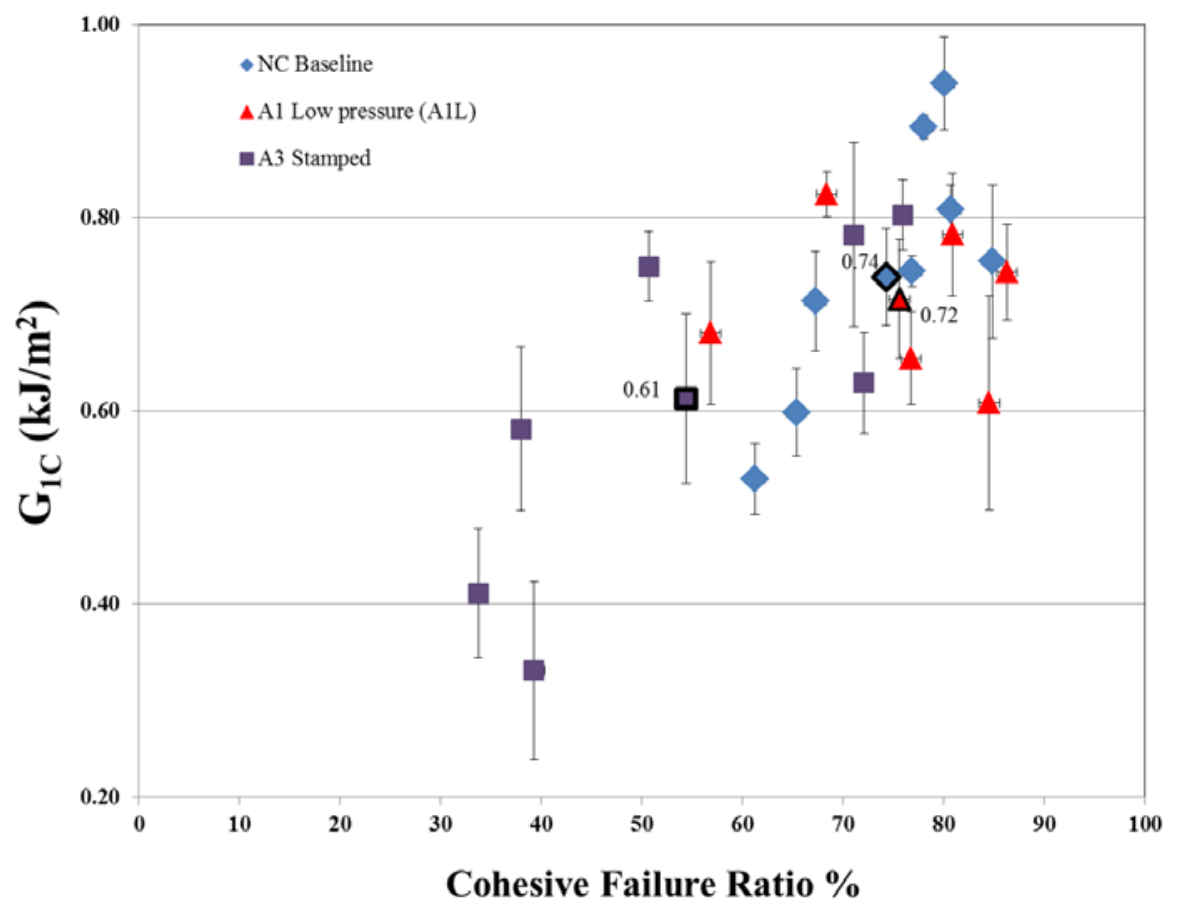

Figure 5.8: GIC vs Cohesive Failure Ratio of Baseline, A1 Stamp and A3 Stamp.

While this marginally showcases the effect of contamination on the bond strength of adhesively bonded joints, A3 contaminated set had a significant influence on fracture toughness with an 
average $\mathrm{G}_{\text {IC }}$ of $0.61 \mathrm{~kJ} / \mathrm{m}^{2}$, reducing it by $17.57 \%$ when compared to the baseline set that had an average of $0.74 \mathrm{~kJ} / \mathrm{m}^{2}$. This is because the amount of contaminant deposited via A3 stamp procedure is predominantly distributed than A1 stamp procedure. The higher the local contaminant concentration, the pronounced is its effect on the bond strength of a joint.

To investigate the effects of total contamination area, only the A1 stamp was used and application pressure was varied. One set of data was obtained using only gravimetric pressure on the stamp (labeled as A1L) and another with approximately $22 \mathrm{~kg}$ of weight placed on the stamp (labeled as A1H). In this case the shape of the applied area (stamp) was constant, but the applied area was increased with the addition of applied pressure on the stamp. As shown in Figure 5.9, similar trends were observed i.e. an increase in the application pressure led to a wider spread of the contaminant affecting larger areas. No fibre bridging was observed for any of the specimens.

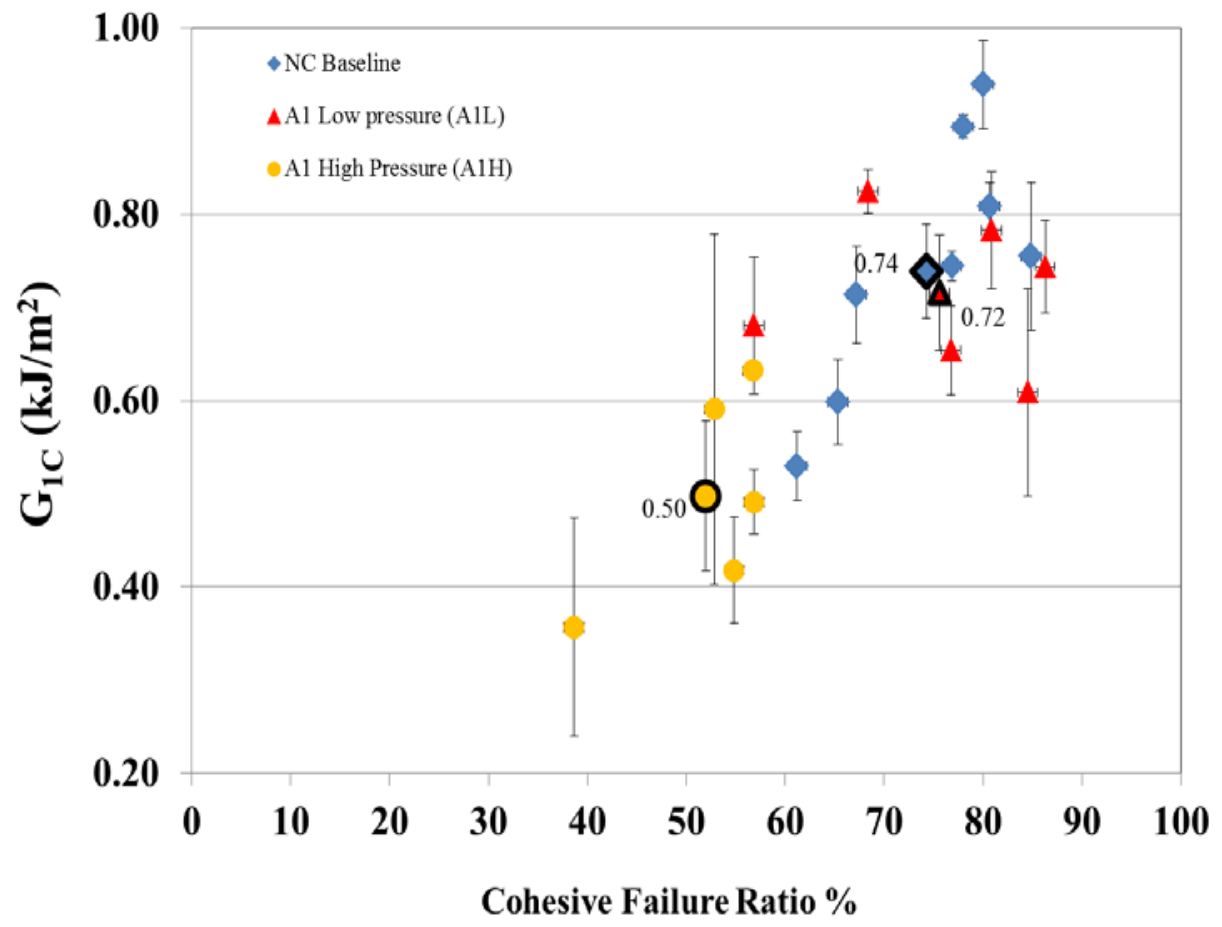

Figure 5.9: $\mathrm{G}_{\text {IC }}$ vs Cohesive Failure Ratio of Baseline, A1 Low Pressure and A1 High Pressure. 
The A1 high pressure specimens had a significant effect on bond strength while A1 low pressure exhibited a marginal degradation in the bond strength. While this marginally showcases the effect of application pressure on the bond strength of adhesively bonded joints, A1H contaminated set had a significant influence on fracture toughness with an average $\mathrm{G}_{\mathrm{IC}}$ of $0.50 \mathrm{~kJ} / \mathrm{m}^{2}$, reducing by $32.4 \%$ when compared to the baseline set with an average $G_{\text {IC }}$ of $0.74 \mathrm{~kJ} / \mathrm{m}^{2}$. The decrease in $G_{1 C}$ is more significant than the decrease observed for the A1L set i.e. $2.8 \%$ due to the contaminant spread as a function of pressure via $\mathrm{A} 1 \mathrm{H}$. The higher the contaminant spread, the more adverse the effect on the bond strength of a joint. Individual failure modes of the specimens were evaluated to further the understanding of fracture with undesirable bonding conditions.

\subsubsection{Modes of Failure}

The individual modes of failure for the all the test cases are examined and shown in Figure 5.10. For the baseline specimens in Figure 5.10a, the mode of failure was observed to be cohesive with minimal interlaminar failure at the edges. Specimens contaminated with the A1 and A3 stamps exhibited mixed mode failure with areas of cohesion failure at non-contaminated sites and adhesive failures at the contaminated sites as shown in Figure 5.10b and c. A1 and A3 category specimens are well in accordance with the average $G_{\text {IC }}$ values indicating an interfacial separation of the adhesive from the composite surface at the localized site which is a clear indication of reduced bond strength. The contaminated site is in-turn surrounded by the adhesive where bonding is strong and a pure cohesive failure is observed. The interlaminar failure at the edges is also observed which is likely due to manufacturing/ cutting effects. In the case of specimens contaminated with $\mathrm{A} 1 \mathrm{H}$, as shown in Figure 5.10d, the effects of pressure are clearly visible with a pure adhesive failure at precrack in 2 specimens while other specimens had adhesive failure at the localized regions with a large affected area. 


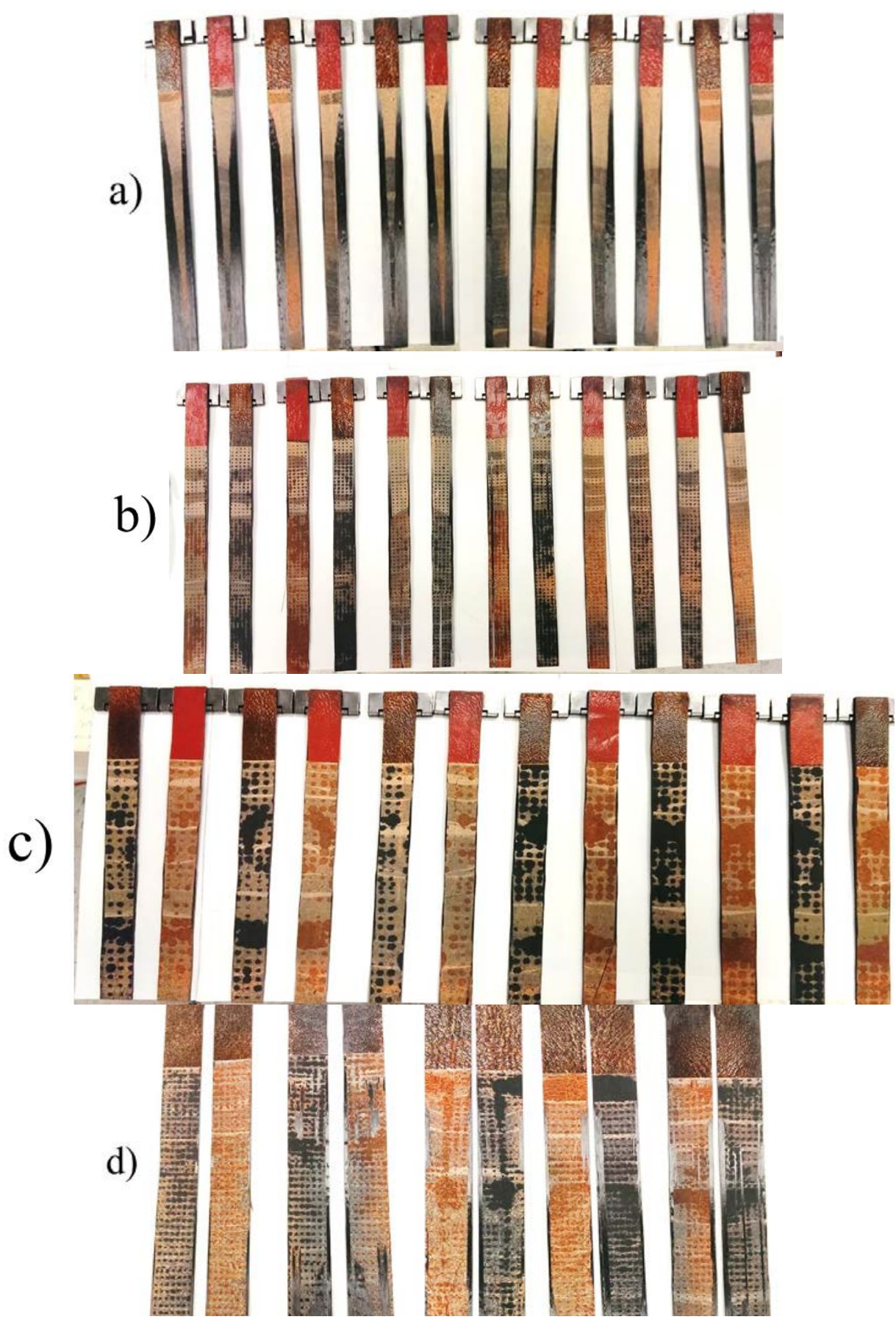

Figure 5.10: Failure Modes a) Baseline (non-contaminated); b) A1 Low Pressure; c) A3 Stamp and d) A1High Pressure 


\subsubsection{Quantification of Failure Modes}

As mentioned in section 5.2, post visual inspection of failure modes was followed by quantifying them. It should be noted that the fracture toughness values were evaluated for the first $5 \mathrm{~cm}$ of the failure region. To have a comparable area measure, quantification was conducted on the identical regions. This will assist in establishing the area correlations that are associated with fracture toughness. A total of 29 digital images were processed through Image J i.e. 8 each for baseline, A1L and A3 specimens while only 5 were manufactured for $\mathrm{A} 1 \mathrm{H}$ specimens due to inventory constraints. In order to avoid repetition of numerous figures, specimens that are a close representative of the average are shown in Figure 5.11.

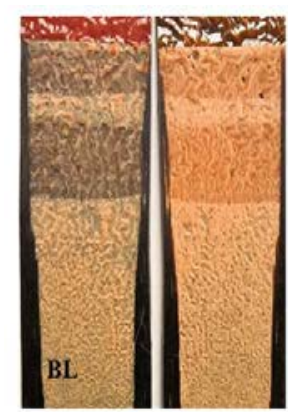

Baseline (no contamination)

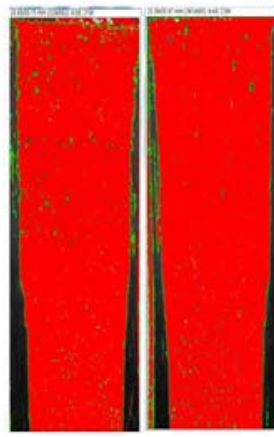

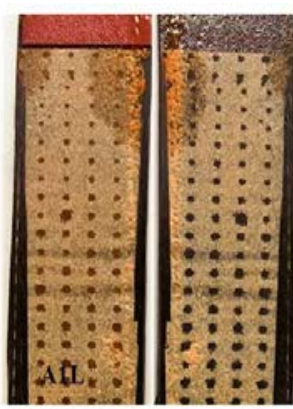

Al contaminated (low pressure)

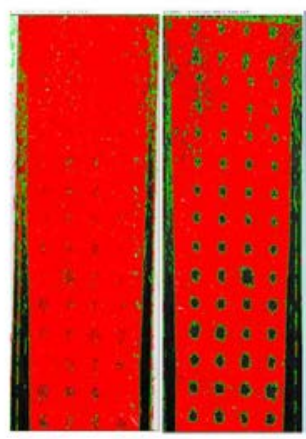

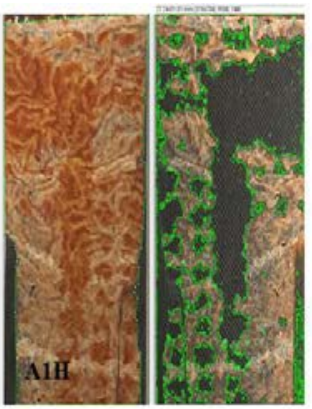

Al contaminated (high pressure)

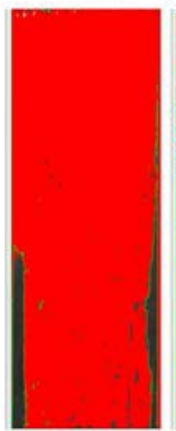

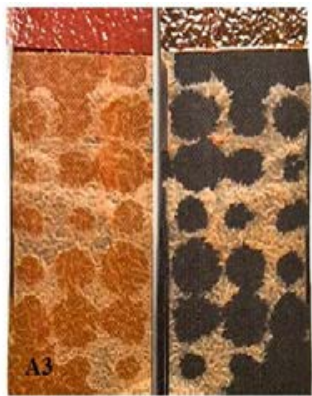

A3 contaminated
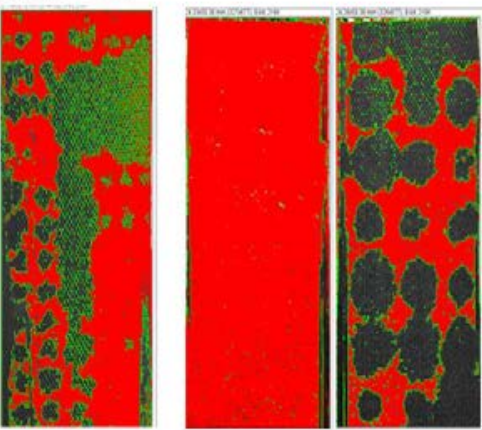

Figure 5.11: Quantified images determining cohesive and adhesive ratios. Top rowfractured surfaces for each of the four configurations. Bottom row- Illustrated computed areas images using ImageJ, red coloration indicates cohesive failure, black indicates adhesive/interlaminar failure, and green demarcates the boundaries between each.

Individual coupons from DCB testing were analyzed and compared to study the influence of overall contaminated area (keeping contaminated size constant) and the influence of contamination size (keeping contaminated area constant). The quantified data fully corroborated 
with the failure modes. Figure 5.12a provides a comparison of two samples contaminated using the A3 procedure (A3-05 vs A3-07), while initial stamp unit cell areas are similar, additional contaminate was applied in the case of A3-07 due to the cutting location on the panel as well as additional spreading during application. There is both a significant difference in cohesive failure ratio (39.3\% for A3-07 vs. $60 \%$ for A3-05) and critical energy release rate $(0.33 \mathrm{~kJ} / \mathrm{m} 2$ for A3-07 vs. $0.7 \mathrm{~kJ} / \mathrm{m} 2$ for A3-05) for these two specimen as a function of contaminate amount, a trend that was repeated throughout analysis as shown in Table 5.2. From these results, it is clear that the contamination procedure was successful in creating a weak bond at the adhesive-composite interface resulting in failure, and increasing levels of contamination resulted in decreased bond quality. Figure 5.12b shows an additional comparison of two samples, one contaminated using

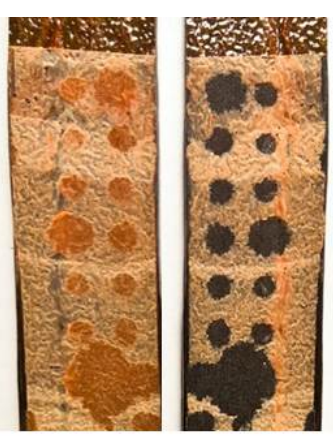

A3-05

$\mathrm{G}_{1 \mathrm{C}}-0.78 \mathrm{~kJ} / \mathrm{m}^{2}$ $\mathrm{COH} \%-71.07$

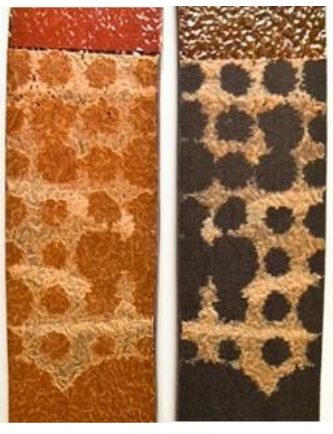

A3-07

$\mathrm{G}_{1 \mathrm{C}}-0.33 \mathrm{~kJ} / \mathrm{m}^{2}$

a) $\mathrm{COH} \%-39.30$

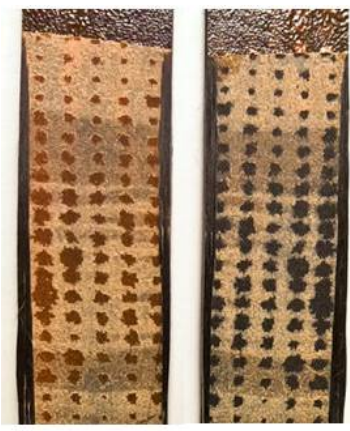

A1L-02

$\mathrm{G}_{1 \mathrm{C}}-0.60 \mathrm{~kJ} / \mathrm{m}^{2}$ $\mathrm{COH} \%-56.85$

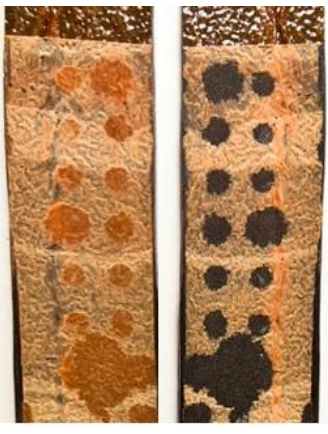

A3-05

b) $\mathrm{G}_{1 \mathrm{C}}-0.78 \mathrm{~kJ} / \mathrm{m}^{2}$

Figure 5.12: Example cases of the effect of (a) contamination area and (b) contamination size on cohesive failure ratio and critical energy release rate values. Contaminated area plays a major role in decreasing the bond quality whereas the effects of contamination size are negligible.

the A1L procedure and one using the A3 procedure (A1L-02 vs A3-05). In this case, the cohesive failure ratios (60\% for A1L-02 to $60 \%$ for A3-05) and critical energy release rates $(0.7 \mathrm{~kJ} / \mathrm{m} 2$ for A1L-02 to $0.67 \mathrm{~kJ} / \mathrm{m} 2$ for A3-05) are very similar, but the sizes of the contaminated regions vary significantly, from a few $\mathrm{mm}$ in A1L-02 to up to $10 \mathrm{~mm}$ in A3-05. These results suggest that the 
critical energy release rate is not significantly affected by a change in contaminate size (A1, A3 etc), but only by the total overall contaminated area.

Table 5.2: Bondline thickness, critical energy release rate (G1C), and cohesive failure ratio of macroscale specimen (DCB) from baseline and contaminated samples

\begin{tabular}{|c|c|c|c|c|c|c|c|c|c|c|}
\hline \multirow[b]{2}{*}{ ID } & \multirow{2}{*}{$\begin{array}{c}\text { Bondline } \\
\text { thickness } \\
\text { [mm] }\end{array}$} & \multirow{2}{*}{ Stamp } & \multirow{2}{*}{$\begin{array}{c}\text { Wt. on } \\
\text { stamp } \\
\text { [kg] }\end{array}$} & \multicolumn{6}{|c|}{$\mathbf{G}_{1 \mathrm{C}},\left[\mathrm{kJ} / \mathrm{m}^{2}\right]$} & \multirow{2}{*}{$\begin{array}{c}\text { Cohesive } \\
\text { Failure } \\
\text { Ratio, } \\
\text { [\%] }\end{array}$} \\
\hline & & & & 1 & 2 & 3 & 4 & 5 & Ave. & \\
\hline BL-01 & 0.24 & --- & --- & 0.79 & 0.69 & 0.66 & 0.69 & -- & 0.71 & 67.23 \\
\hline BL-02 & 0.23 & --- & -- & 0.55 & 0.56 & 0.53 & 0.46 & & 0.52 & 61.22 \\
\hline BL-03 & 0.31 & --- & --- & 0.53 & 0.63 & 0.65 & 0.61 & 0.55 & 0.59 & 80.68 \\
\hline BL-04 & 0.35 & --- & --- & 0.73 & 0.76 & 0.72 & 0.74 & -- & 0.74 & 76.86 \\
\hline BL-05 & 0.40 & --- & -- & 0.77 & 0.83 & 0.79 & 0.82 & -- & 0.80 & 65.34 \\
\hline BL-06 & 0.38 & --- & --- & 0.96 & 0.98 & 0.99 & 0.93 & 0.89 & 0.93 & 80.05 \\
\hline BL-07 & 0.40 & --- & --- & 0.88 & 0.89 & 0.88 & 0.89 & 0.88 & 0.89 & 78.01 \\
\hline BL-08 & 0.37 & --- & -- & 0.62 & 0.77 & 0.71 & 0.81 & 0.84 & 0.75 & 84.86 \\
\hline P-Ave. & 0.34 & --- & --- & & & & & & 0.74 & 74.28 \\
\hline A1L-01 & 0.24 & $1 \mathrm{~mm}$ & $\sim 0$ & 0.64 & 0.70 & 0.58 & 0.68 & -- & 0.65 & 76.76 \\
\hline A1L-02 & 0.25 & $1 \mathrm{~mm}$ & $\sim 0$ & 0.73 & 0.74 & 0.52 & 0.50 & 0.52 & 0.60 & 56.85 \\
\hline A1L-04 & 0.33 & $1 \mathrm{~mm}$ & $\sim 0$ & 0.85 & 0.83 & 0.78 & 0.81 & 0.81 & 0.82 & 86.28 \\
\hline A1L-05 & 0.29 & $1 \mathrm{~mm}$ & $\sim 0$ & 1.2 & 0.93 & 0.94 & -- & -- & 1.02 & 84.52 \\
\hline A1L-06 & 0.25 & $1 \mathrm{~mm}$ & $\sim 0$ & 0.82 & 0.88 & 0.75 & 0.71 & 0.73 & 0.78 & 68.38 \\
\hline A1L-07 & 0.26 & $1 \mathrm{~mm}$ & $\sim 0$ & 0.79 & 0.76 & 0.67 & -- & -- & 0.74 & 80.85 \\
\hline $\begin{array}{l}\text { A1L- } \\
\text { Ave. }\end{array}$ & 0.27 & $1 \mathrm{~mm}$ & $\sim 0$ & & & & & & 0.72 & 75.61 \\
\hline A1H-01 & 0.23 & $1 \mathrm{~mm}$ & $\sim 22$ & 0.39 & 0.49 & 0.44 & 0.72 & 0.89 & 0.59 & 52.83 \\
\hline A1H-02 & 0.35 & $1 \mathrm{~mm}$ & $\sim 22$ & 0.62 & 0.63 & -- & -- & -- & 0.63 & 56.71 \\
\hline A1H-03 & 0.41 & $1 \mathrm{~mm}$ & $\sim 22$ & 0.50 & 0.44 & 0.52 & -- & -- & 0.49 & 56.82 \\
\hline A1H-04 & 0.38 & $1 \mathrm{~mm}$ & $\sim 22$ & 0.19 & 0.46 & 0.41 & -- & -- & 0.35 & 38.65 \\
\hline A1H-05 & 0.26 & $1 \mathrm{~mm}$ & $\sim 22$ & 0.39 & 0.36 & 0.49 & -- & -- & 0.41 & 54.84 \\
\hline $\begin{array}{l}\text { A1H- } \\
\text { Ave. }\end{array}$ & 0.33 & $1 \mathrm{~mm}$ & $\sim 22$ & & & & & & 0.50 & 51.97 \\
\hline A3-01 & 0.18 & $3 \mathrm{~mm}$ & $\begin{array}{l}\sim 0 \\
\sim\end{array}$ & 0.64 & 0.68 & 0.56 & -- & -- & 0.62 & 72.07 \\
\hline A3-02 & 0.22 & $3 \mathrm{~mm}$ & $\sim 0$ & 0.42 & 0.62 & 0.56 & 0.63 & 0.66 & 0.58 & 37.96 \\
\hline A3-03 & 0.29 & $3 \mathrm{~mm}$ & $\sim 0$ & 0.75 & 0.81 & 0.83 & -- & -- & 0.80 & 75.93 \\
\hline A3-04 & 0.30 & $3 \mathrm{~mm}$ & $\sim 0$ & 0.78 & 0.71 & -- & -- & -- & 0.74 & 50.67 \\
\hline A3-05 & 0.33 & $3 \mathrm{~mm}$ & $\sim 0$ & 0.75 & 0.90 & 0.68 & -- & -- & 0.78 & 71.07 \\
\hline A3-06 & 0.34 & $3 \mathrm{~mm}$ & $\sim 0$ & 0.34 & 0.47 & -- & -- & -- & 0.41 & 33.72 \\
\hline A3-07 & 0.34 & $3 \mathrm{~mm}$ & $\sim 0$ & 0.24 & 0.45 & 0.29 & -- & -- & 0.33 & 39.30 \\
\hline $\begin{array}{l}\text { A3- } \\
\text { Ave. }\end{array}$ & 0.28 & $3 \mathrm{~mm}$ & $\sim 0$ & & & & & & 0.61 & 54.38 \\
\hline
\end{tabular}




\subsubsection{Fractographic Analysis}

An optical microscope equipped with a line profiling feature was utilized to investigate the failure modes obtained after mechanical testing. The ultimate aim is to understand the fracture/crack profile and its topography which assists us in determining the fracture path that occurred during fracture. The non-contaminated specimen and A1 and A3 contaminated specimen are shown in Figure 5.13a, b and c, respectively. A pure cohesive failure is observed in noncontaminated site as seen in Figure 5.13a while at the A1 and A3 contaminant sites as seen in Figure 5.13b, the adherend surface woven pattern is visible which can be attributed to reduced adhesiveadherend crosslinking during bonding process as explained in section 4.2.2.3. A pure adhesive failure is apparent at the contaminated site whereas a pure cohesive failure is apparent at the remaining regions (non-contaminated site).

A line profile is drawn on this failure surface to understand the crack propagation profile which is shown at the bottom of their respective datasets. The profile of the line if drawn on a noncontaminated site clearly indicates the cohesion path of the crack i.e. crack traverses within the adhesive. While in the contaminated set, the hilly regions correspond to the adhesive and the valleys correspond to the substrate or the prepreg (no adhesion region).

Spectral image mapping of the fracture profile was conducted post line profile analysis. A spectral map functions with the contrast of the line profile is shown at the bottom of the Figure 5.13. In non-contaminated specimens, the spectral distribution is even with grey regions (near white) indicating the adhesive alone. While the spectral map for the contaminated set, clearly discerns the white and black regions along the fracture profile which corresponds to the adhesive and the adherend, respectively. This shows a mixed mode type failure with cohesion and adhesion as intermediates for the crack traversing path. 

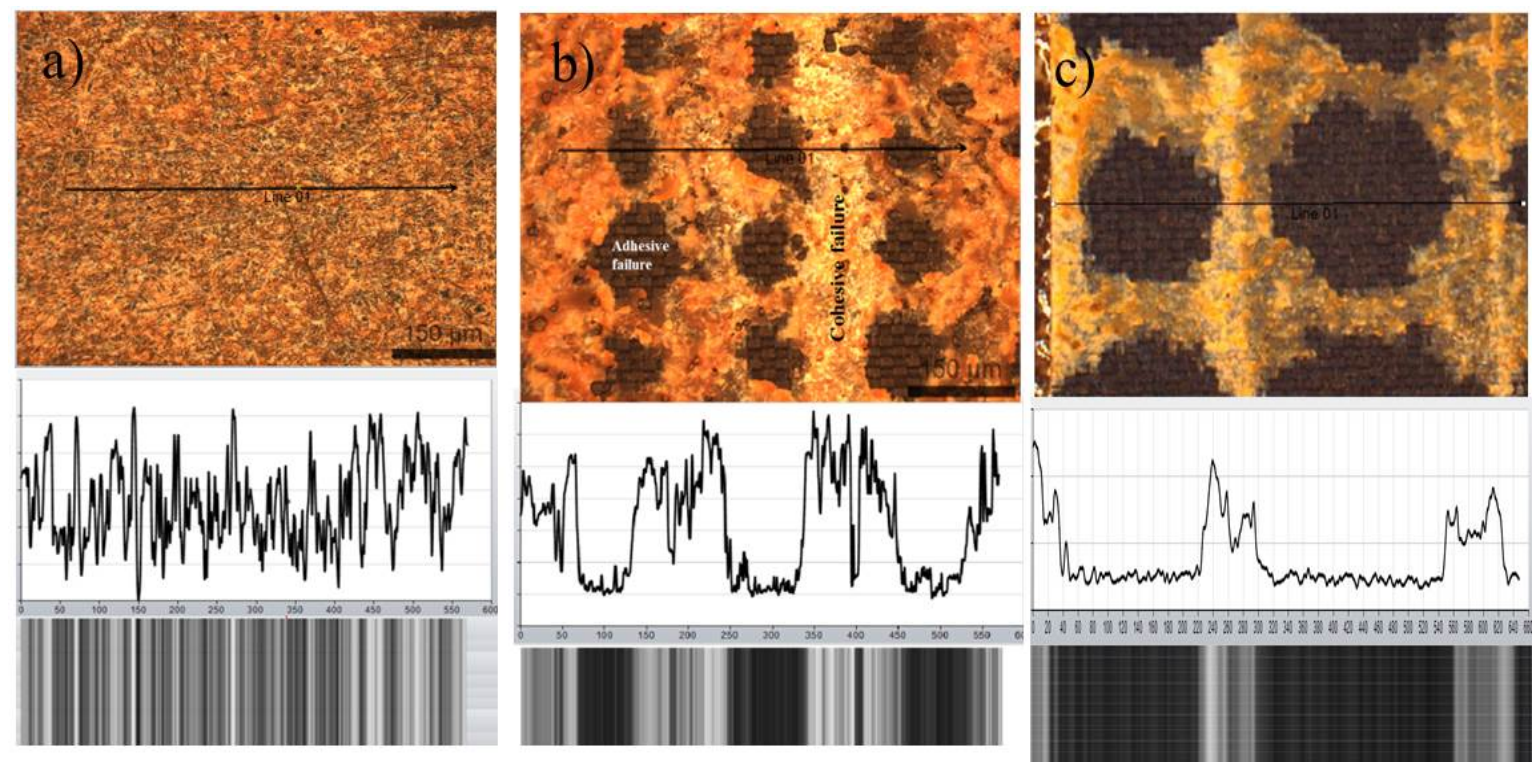

Figure 5.13: Line profile analysis showing a line drawn over the fractured surface a) baseline specimen; b) A1L stamp specimen and c) A3 stamp specimen. Line profile path is shown beneath the optical image follwed by their spectral mapping.

\subsection{Conclusions}

In this study the influence of surface preparation on composite-adhesive bond quality was investigated through the development of a repeatable and scalable surface contamination procedure. As the strength of adhesively bonded joints continue to increase with improved chemistry, possible new applications for adhesively bonded composite joints become viable, and as such it is important to understand the additional factors that may influence bond performance.

The macroscale DCB testing and in situ micro scale ENF analysis showed a direct correlation between locations of contaminate application and the initiation of damage. In addition, overall contaminated area plays a major role in decreasing the bond quality whereas the effects of contamination size were not pronounced. The line profile analysis revealed the nature of crack path and helped in understanding the role of contamination in governing the fracture behavior.

The results of this study can be used to improve the design of adhesively bonded composites and to design NDE techniques to assess bond quality prior to implementation. For instance, hand-held FTIR scans can be used at various stages of composite processing/composite 
bonding to determine the presence of contaminate locations and overall contaminated area. Standards for acceptable amount of contamination can focus solely on the total contaminated area and not on the shape or morphology of the contaminated region. In addition, future design can incorporate direct evidence of the influence of weak bonds on overall performance in design and modeling software, leading to enhanced confidence in predictions and a reduction in overall component weight and in the need for arbitrarily high safety factors or overdesigned mechanical fasteners. 


\subsection{References}

[1] Y. Zhang, A. P. Vassilopoulos and T. Keller, "Mode I and II fracture behavior of adhesively bonded pultruded composite joints," Engineering Fracture Mechanics, vol. 77, pp. 128-143, 2010 .

[2] ASTM D3433-99, "Standard test method for fracture strength in cleavage of adhesives in bonded metal joints," in Annual book of ASTM Standards;, 2005, p. in adhesive section 15.06 .

[3] ASTM D5528-01, "Standard test method for mode I interlaminar fracture toughness of unidirectional fiber-reinforced polymer matrix composites," in Annual book of ASTM Standards, 2007, p. in adhesive section 15.03.

[4] ISO 15024:2000(E), "Standard test method for the mode I interlaminar fracture toughness, GIIC, of unidirectional fibre-reinforced polymer matrix composites," 2000.

[5] ESIS Protocol, Determination of the mode II delamination resistance (Critical Energy Release Rate GIIC) of unidirectional fiber-reinforced polymer laminates using the endloaded-split specimen (ELS), 2006.

[6] A. Brunner, B. Blackman and G. Williams, "Calculation of a damage parameter and bridging stress from GIC delaminating tests on fibre composite.," Composite Science and Technology, vol. 66, no. 6, pp. 785-795, 2006.

[7] A. Hodzic, K. Kim, A. Lowe and Z. Stachurski, "The effects of water aging on the interphase region and interlaminar fracture toughness in polymer-glass composites," Composite Science and Technology, vol. 64, no. 13-14, pp. 2185-95, 2004.

[8] C. Thanomsilp and P. Hogg, "Interlaminar fracture toughness of hybrid composites based on commingled yarn fabrics," Composite Science and Technology, vol. 65, no. 10, pp. 15471563, 2005.

[9] M. Hecker, R. Hentschel, M. Hensel and M. U. Lehr, "Crack propagation and delamination analysis within the die by camera-assisted double cantilever beam technique," in Interconnect Technology, IEEE International Conference - IITC, 2011.

[10] S. Mall, "Characterization of debond growth mechanism in adhesively-bonded composites under mode II static and fatigue loadings," Engineering Fracture Mechanics, vol. 31, no. 5, pp. 747-58, 1988.

[11] R. Martin and B. Davidson, "Mode II fracture toughness evaluation using four point bend, end notched flexure test," Plastics, Rubber and Composites, vol. 28, no. 8, pp. 401-406, Aug 1999. 
[12] A. Pocius, D. Dillard and M. Chaudary, Adhesion Science and Engineering, Amsterdam: Elsevier, 2002.

[13] M. Davis and J. Tomblin, "Best Practice in Adhesive bOnded Structures and Repairs," U.S. DOT, Federal Aviaiton Administration (FAA), Seattlte WA., April 2007.

[14] M. Davis and A. McGregor, "Assessing Adhesive Bond Failure: Mixed Mode Bond Failures Explained," in ISASI Australian Safety Seminar, Canberra, June 2010. 


\section{DURABILITY OF ADHESIVELY BONDED COMPOSITE JOINTS}

Adhesive bond durability can be defined as bond's ability to preserve its initial strength upon subjecting it to various loading conditions or any external harsh environmental conditions over time. The dearth of knowledge on dependable test methodologies and accelerated aging procedures or predictable approaches for determining the durability of structural adhesive joints has impeded the universal acceptance of adhesive bonding [1]. This in conjunction with the remotely applied stresses can exacerbate the structural performance and failure. A combination of stress factors along with severe environmental conditions can generally result in overestimating the strength of the designed bonded joint. Therefore, the acceptable level of safety that is governed by the strength and durability of a bonded structure is of paramount importance to manufactures and consumers and needs to be defined/determined serving as a qualification level with regards to long term strength of an ABCJ. In this chapter, emphasis on developing a test methodology to assess durability is demonstrated. Non-contaminated and contaminated specimens were subjected to a developed durability procedure and their effects on bond strength were characterized. Moisture absorption studies were conducted simultaneously on composite laminates and on adhesively bonded composites to monitor the effects of environment on the material system.

\subsection{Development of Durability Test Procedure}

Understanding the long term performance of ABCJ's i.e. mechanical as well as other physical property effects, as a function of time is of prime importance in this study. This starts with developing a testing protocol that can reliably evaluate the durability of ABCJ's. Based on information obtained in the literature review, bond durability assessment would optimally utilize testing with specimens that have been preconditioned in harsh environmental conditions or mechanically loaded fatigue limits. The preconditioning of specimens prior to fracture tests, should emulate the conditioning of bonded components in service. The utilization of this type of conditioning presents challenges in establishing an appropriate conditioning protocol. The 
harmonization between the existing methods such as environmental exposure or mechanical loading at high temperatures is less than satisfactory for the current status of testing durability of ABCJ's.

The proposed methodology focuses on using DCB coupons that are conditioned in ways that are similar to service conditions. Four sets of conditions will be used and are listed below.

(a) baseline specimen (non-conditioned)

(b) environmentally conditioning for elapsed times

(c) fatiguing in ambient air

(d) combined environmental exposure and fatiguing simultaneously

After conditioning, the DCB coupons will be tested to evaluate fracture toughness. $G_{\text {IC values for }}$ each set will be determined and comparisons will be made with baseline specimens and

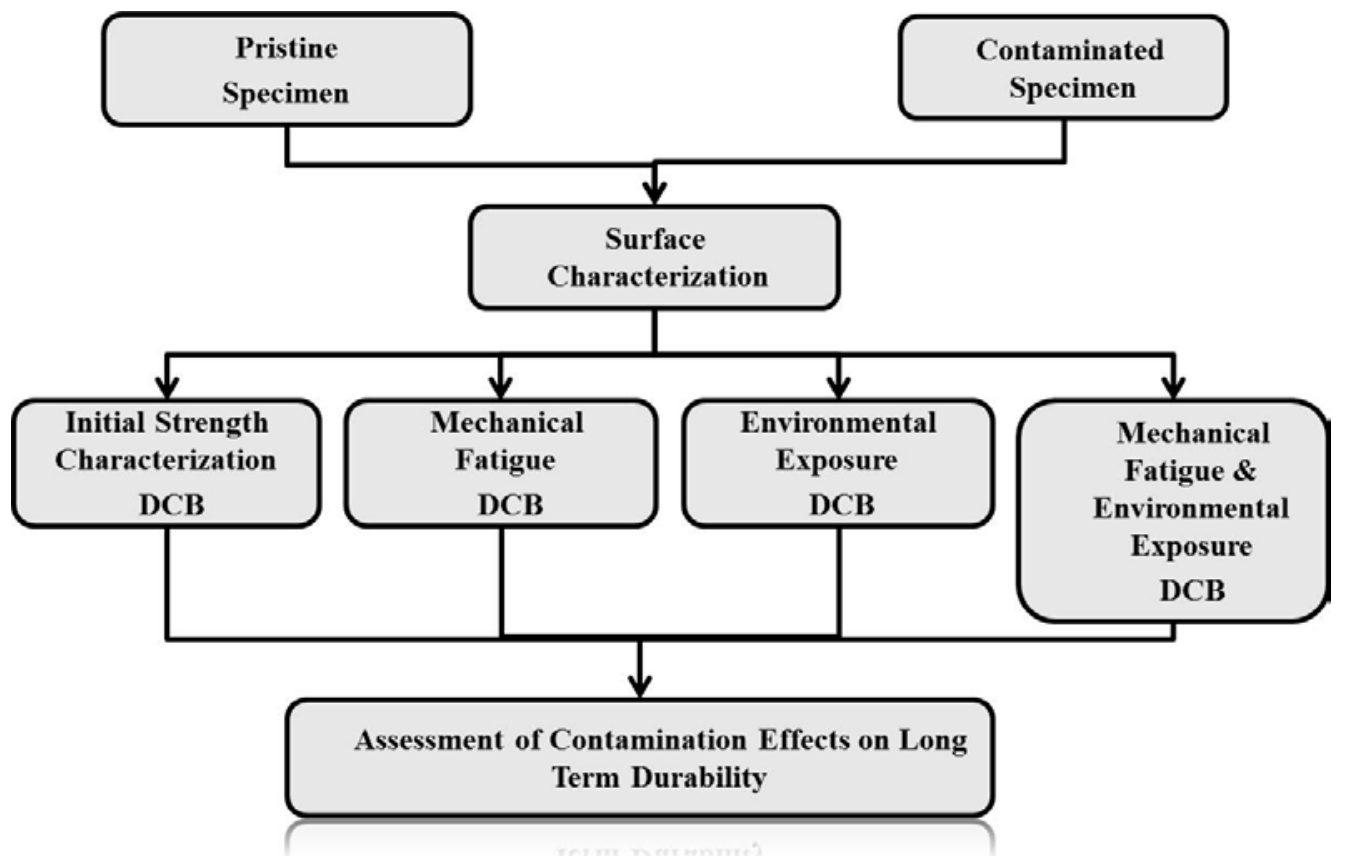

Figure 6.1: Durability Assessment Procedure 
contaminated specimens. Analysis of the test cases will be conducted and trends will be established. This type of approach allows for various surface preparation methods to be investigated as well as the effect of bonding with Undesirable Bonding Conditions (UBC) i.e. contamination on long-term durability. In order to minimize the variability of several influencing factors, exposure temperature, exposure time and relative humidity have been kept constant as the projects ultimate goal is to evaluate the effects of undesirable bonding conditions on durability. For the environmentally exposed specimens, the exposure time was initially chosen to be 2 months with an exposure temperature of $50^{\circ} \mathrm{C}$ and $95 \% \mathrm{RH}$. These aging conditions are more severe than those expected to occur in service as they are accelerated tests and are selected in order to quantify the changes within a reasonable exposure time. The schematic of the proposed methodology is shown above in Figure 6.1. The mechanical loading will utilize a composite bonded specimen geometrically similar to those found in ASTM D790. The specimens will be placed in a novel fixture that supplies support at the coupon ends and is cyclically loaded at the center using a pneumatic cylinder to control the force/displacement that is imposed. This will allow for the use of different test frequencies and amplitudes. The magnitude of the shear load distribution of the specimen will remain constant along the specimen and the tension or compression loads at the bondline approaches zero. This configuration challenges the interlaminar shear strength at the centroid of the specimen and can be adapted to fit within an environmental chamber.

On the other hand, it is noteworthy to investigate the effects of harsh environments for extended periods of time in an unstressed conditions. This assists in an improved understanding of the behavior of moisture over a period of time and is discussed in the section 6.1.1.

\subsubsection{Moisture Absorption Studies of Composite Laminates and ABCJ's}

For many years it has been known that epoxy bonded joints, upon exposure to humid environments, have a significant impact on its bond performance [4, 5, 6]. Orman and Kerr [7] reported significant joint strength degradation and harmful effects of environment on epoxy bonded 
aluminum joints. Epoxy based adhesives were dominantly utilized in joining metals for aerospace applications [6]. Many of the moisture uptake studies on composite laminate or only adhesives showed degradation in mechanical performance [8], Saponara [9] investigated in understanding the diffusion kinetics of structural adhesives and composite laminates where two models were developed to predict the durability of polymeric composite materials and neither models were capable to fit data for the adhesive mass uptake. Substantial swelling of the materials was reported yet marginal degradation behavior was noticed. While degradation behavior has generally been observed, there has been a very little agreement for moisture uptake within metal bonded joints itself. Bowditch [10] investigated the durability of aluminum adhesive joints in the presence of water and reported an initial increase in joint strength. This was attributed to plasticization within the adhesive. Similar responses with respect to ABCJ's were documented. Selzer and Friedrich [10] investigated the moisture absorption characteristics of bonded composites where the specimens were immersed in a water baths for various temperatures and found their $G_{1 C}$ values higher than the dry specimens (baseline). In other work of Selzer and Friedrich [11], fracture toughness increased with increasing moisture content. Similar observations were observed by Lucas and Zhou in their work on bonded composite joints [13]. Pantelakis and Tserpes [15] subjected bonded composite specimens to 4200 hours with an $\mathrm{RH} 85 \%$ at $70^{\circ} \mathrm{C}$ and found an increase in toughness of a bonded joint. Katsiropoulos et al. [16] found similar behaviors of increased toughness upon wet ageing with an adhesive bondline of $0.5 \mathrm{~mm}$. These articles suggest that the ABCJ's have very complex material systems and are difficult to understand when extrinsic factors such as moisture ingression or thermal effects are used as stressors.

Manufactured specimens selected for moisture absorption studies were placed in an environmental chamber and care was taken that no two specimens coexisted side by side. For this section the same environmental conditions mentioned in the previous section are used, 95\% RH and $50^{\circ} \mathrm{C}$. Subsequent weight measurements of the specimens were taken on a weekly basis to 
monitor their weight gain until the sorption equilibrium criterion has been reached. The percentage moisture uptake was calculated as:

$$
\% \text { Moisture Uptake }=\frac{\left(M_{i}-M_{O}\right)}{M_{O}} \times 100
$$

where, $M_{i}$ is the final weight of specimen after exposure, $M_{o}$ is the initial weight of specimen before exposure. A total of 16 specimens were selected, of which 8 specimens are bonded with the adhesive film and the other eight are joined without the adhesive film as shown in Figure 6.2. These specimen types were selected to determine the water sorption on the laminate alone and on the adhesive.

All specimens were stored in a dry and environment weighed to the nearest $0.0001 \mathrm{mg}$. Specimens where then placed in the environmental chamber as shown in Figure 6.2. Out of the 8 specimens, four were pulled out after two months to test for their fracture strength and observe the influence of moisture uptake on the non-bonded and bonded specimens. The absorption behavior as a function of time represents Fickian behavior of linear mass uptake [2, 3].

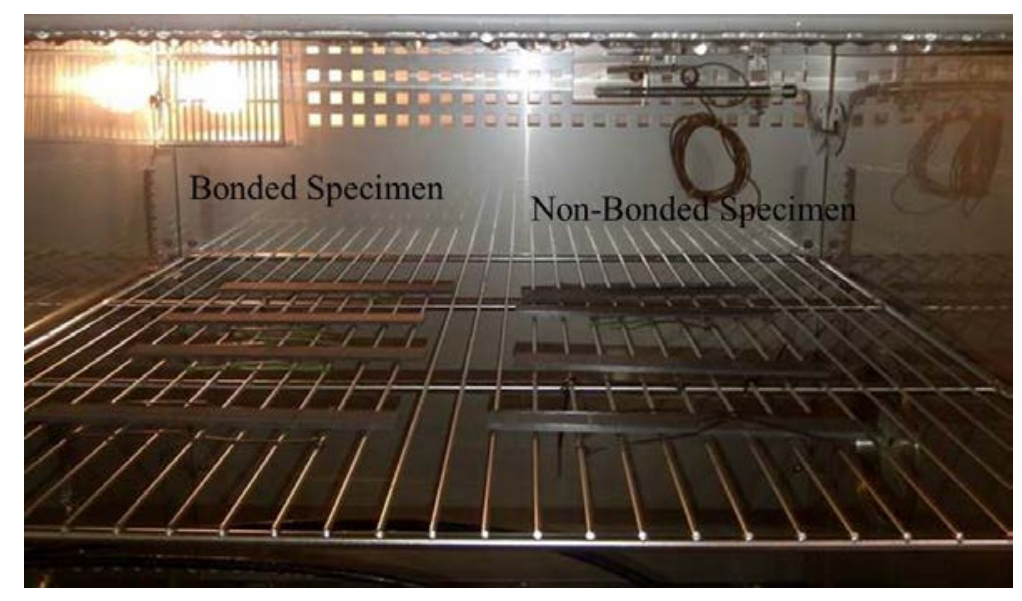

Figure 6.2: Bonded and Non-bonded specimen placed in an environmental chamber for moisture uptake analysis.

\subsection{Experimental Methodology}




\subsubsection{Environmental Aging}

Comparison of non-contaminated, A1 and A3 contaminated specimens on fracture toughness of ABCJ's using accelerated environmental conditions are being made in this section. In general, a tropical based supersonic aircraft, experiences a specific relative humidity $(\mathrm{RH})$ of around $81 \%$. A use of 95\% RH will assist in data extrapolations when test specimens are conditioned to equilibrium at that moisture level. This will aid in assessing the extreme boundary conditions and facilitates the need for an investigation on the effect of environmental aging on the bonded assembly. Specimens for this study are environmentally aged in a Thermotron 2800 Environmental chamber in an unstressed condition as shown in Figure 6.2 and Figure 6.3. A total of 8 specimens are subjected to harsh environments i.e. controlled temperature and humidity conditions at $50^{\circ} \mathrm{C}$ and $95 \% \mathrm{RH}$. The fluid selected for the aging process is di-ionized water.
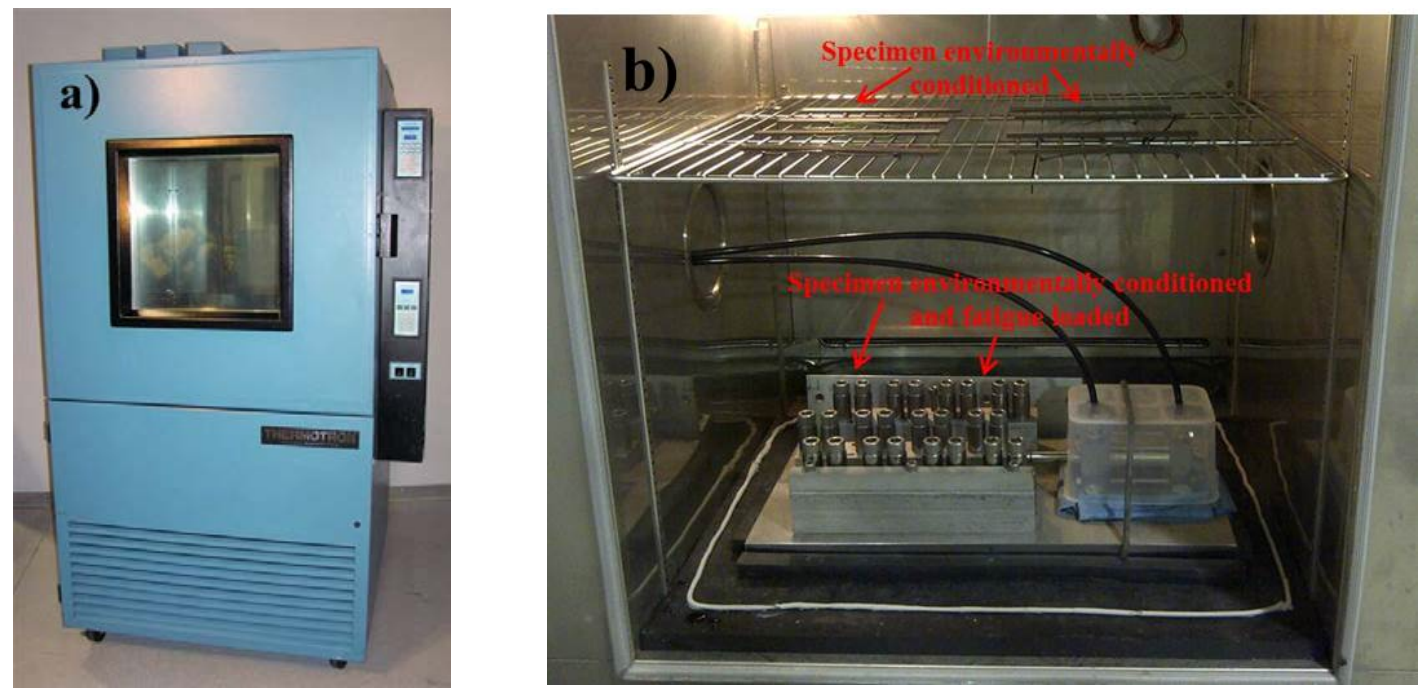

Figure 6.3: a) Environmetal chamber on left and b) Inside an environmental chamber

\subsubsection{Fatigue}

Fatigue of the specimens is accomplished using a cyclic three point bending load as shown in Figure 6.4 where a uniform shear can be created at the bondline. Several design considerations have been taken into account to minimize the surface stress effects, prior to fatiguing the bonded 
specimens. Numerous iterations have been conducted for the nominal thickness considering the properties as explained in section 3.3.2.

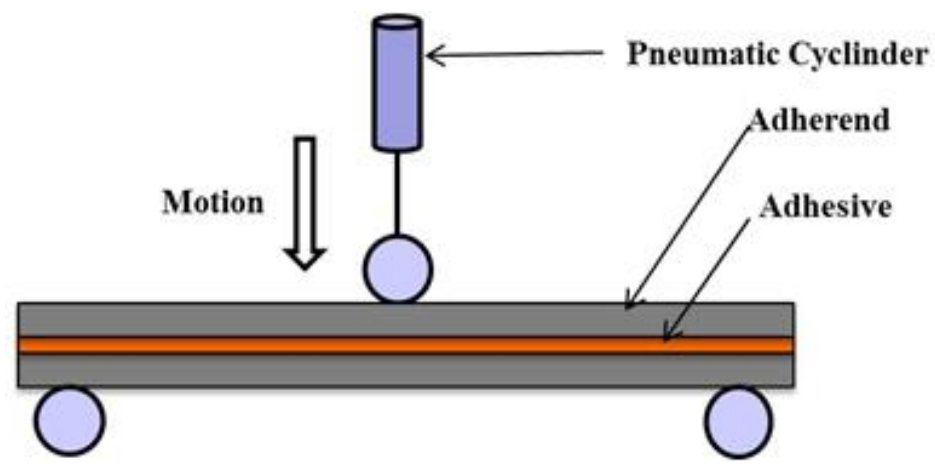

Figure 6.4: Schematic set-up of the fatigue fixture.

To obtain the uniform shear stress, a fatigue fixture was designed to mechanically load DCB specimens with a fully reversible three point bending type load (Figure 6.5). By orchestrating the design of the DCB specimen in conjunction with the design of the fixture, it was possible to determine the specimen dimensions and fixture displacement/load parameters that will provide a close-to-even shear load along the bondline without exceeding the specimens tensile and bending yield parameters. The bending of the specimens is caused by a hydraulic powered piston driven by computer controlled rapid acting valves. This allows the use of different loading frequencies, pressures and pressure profiles. The fatigue fixture was designed to provide a 1 inch maximum deflection at half the length of each specimen. It was determined that this configuration provides a relatively uniform shear load at the bondline of approximately $3000 \mathrm{psi}$, requiring $330 \mathrm{lb}$ of force per specimen. The fixture operates with a double amplitude fatigue loading frequency between 1 $\mathrm{Hz}$ and $0.25 \mathrm{~Hz}$. During the fatiguing process, the loading frequency is adjusted to produce a total of 2.8 million cycles on a 2 month period. The number of cycles is calculated in the specimen design table described in section 3.3.2. 

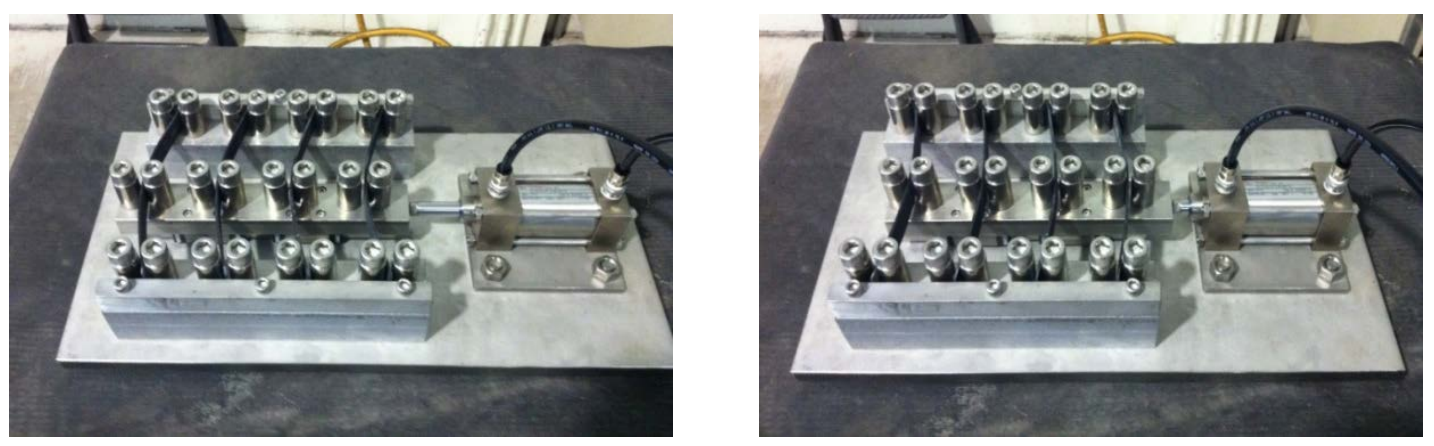

Figure 6.5: Fatigue fixture for DCB specimens- specimen loaded with 0.5 inch a) forward amplitude; and b) backward amplitude.

This fatigue loading will utilize a composite bonded specimen geometrically similar to those found in ASTM D790 (Standard Test Methods for Flexural Properties of Unreinforced and Reinforced Plastics and Electrical Insulating Materials). The magnitude of the shear load remains constant along the specimen length and the tension and compression loads at the bondline approach zero. This configuration challenges the interlaminar shear strength at the centroid of the specimen. A total of 4 specimens can be loaded into the device and fatigued simultaneously as shown in Figure 6.5. Fatigue conditioning is conducted for non-contaminated sets, A1 and A3 contaminated sets with the results provided in the results and discussion section.

\subsubsection{Combined Environmental Aging and Fatigue}

In addition to exposing specimens to an accelerated aging environment to, other specimens were environmentally aged and cyclically loaded simultaneously. A total of four specimens were conditioned for a period of two months at $50^{\circ} \mathrm{C}$ and $95 \% \mathrm{RH}$. The fatigue fixture was developed to fit inside the environmental chamber in order to conduct accelerated aging and fatiguing simultaneously as shown in Figure 6.6. To create the fatigue load, the cylinder pressure is oscillated between 0 and 120 psi at the pre-stated frequency. This pressure produces a total specimen deflection of 0.5 in (total amplitude of 1.0 in at the center of the specimens). The Toray 800 (P2362W-19U-304) prepreg has an ultimate strength of 212×10³ psi which theoretically should be able to sustain a 1 inch deflection with the current specimen design. The 1 inch deflection during 
fatigue loading keeps the specimen loads below the nominal failure limits for the desired number of cycles (see section 3.3.2).

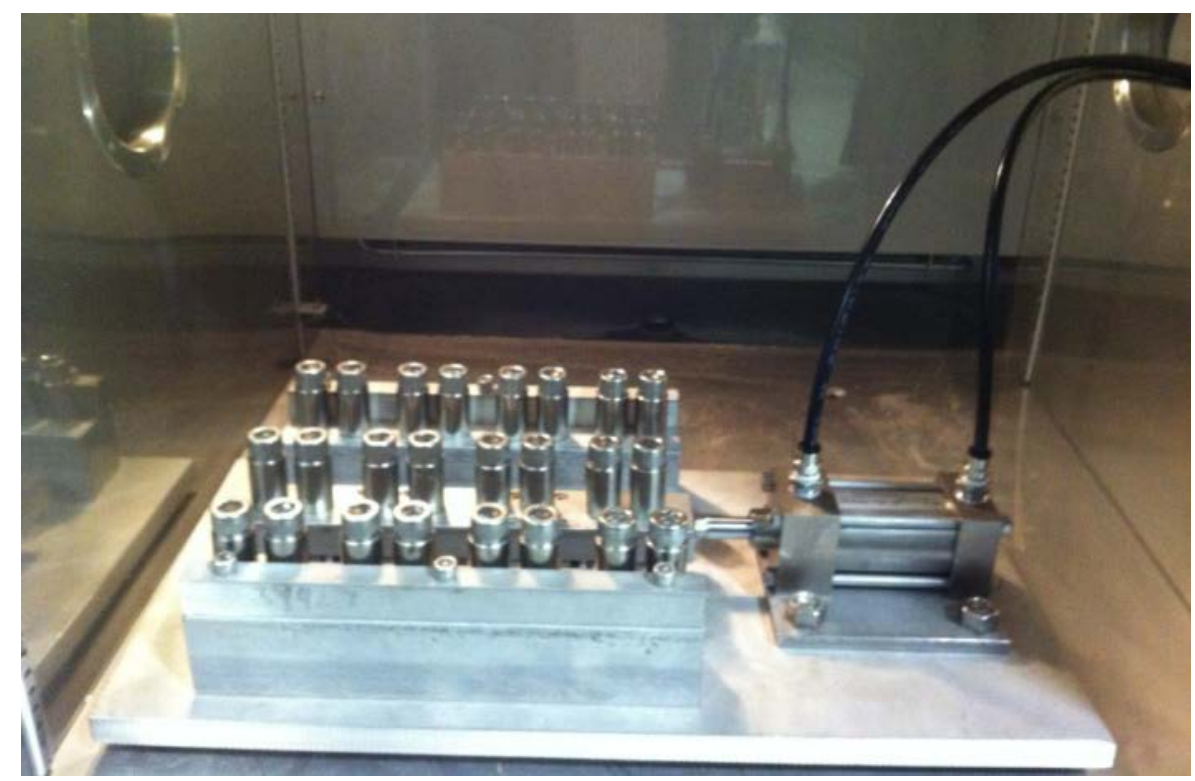

Figure 6.6: Fatigue fixture inside an environmental chamber.

\subsection{Results and Discussions}

\subsubsection{Mechanical Strength Characterization for Durability Specimens}

\subsubsection{Environmental Aging}

Non-contaminated and contaminated specimens conditioned in the environmental chamber for 2 months in an unstressed condition and changes in their mechanical strength was characterized via changes in fracture toughness. Strain energy release rates associated with crack growth were calculated as per ASTM D5528 and as mentioned in section 5.1.1. The $\mathrm{G}_{\text {IC }}$ values for the environmentally conditioned non-contaminated and contaminated specimens are shown in Figure 6.7. For a 2 month exposure time, marginal variability in $\mathrm{G}_{\mathrm{IC}}$ was observed for the environmentally exposed specimens. For the A1 contaminated specimens, only a small reduction in fracture toughness ( $10 \%)$ was observed when compared to the non-contaminated specimens. The average $\mathrm{G}_{\text {IC }}$ value associated with non-contaminated specimens was $0.74 \mathrm{~kJ} / \mathrm{m}^{2}$, while the A1 contaminated specimens exhibited $0.67 \mathrm{~kJ} / \mathrm{m}^{2}$. This demonstrates marginal effects of contamination on 
environmental aging, A3 contaminated specimens exhibited an inverse effect with an increase in their fracture toughness. The average $\mathrm{G}_{\mathrm{IC}}$ value was $0.85 \mathrm{~kJ} / \mathrm{m}^{2}$, which showed an increase $(\sim 13 \%)$ when compared to the baseline set. when compared to the baseline set. This is possibly attributed to the crack bluntness of A3 contaminated specimens.

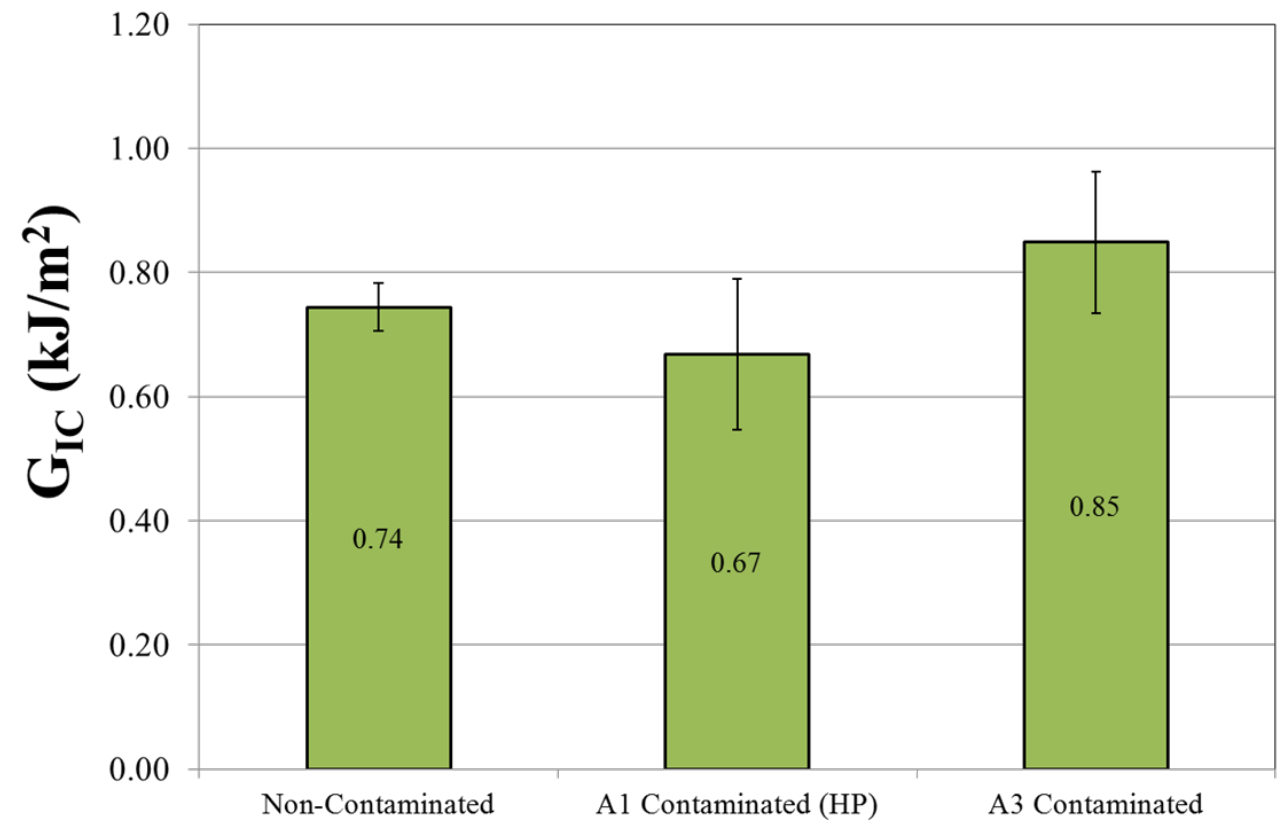

Figure 6.7: Non-Contaminated and Contaminated specimen subjected to Environmental Exposure

A larger localized contaminated site can accumulate larger stresses at the crack tip before a failure occurs or crack propagates. This could also translate a ductile fracture to a brittle fracture. Joshi et al. [17] found that a certain amount of water within a bond actually increases the amount of load it can withstand prior to fracture due to plasticization of the adhesive. This suggests that although contamination can degrade the bond, upon environmentally ageing with water for the exposure time adopted i.e. 2 months had no effect on the fracture toughness of the joint.

\subsubsection{Fatigue in Ambient Air}

Non-contaminated and contaminated specimens were fatigued in ambient air to understand the effect of fatigue alone. The parameters that control cyclic loading (see section 6.2.2) were kept under the failure limit and the desired number of cycles was adjusted to reach the near failure limit 
for a time period of 2 months. This emulates the conditioning for mechanical fatiguing. The DCB coupons post-fatigue were characterized for their mechanical strength by determining their fracture toughness. The strain energy release rates associated with crack growth were calculated as per ASTM D5528 and as mentioned in section 5.1.1. The $\mathrm{G}_{\mathrm{IC}}$ values for the mechanically fatigued non-contaminated and contaminated specimens are shown in Figure 6.8. For the fatigue of noncontaminated and A1 contaminated set, contamination did not have any effect on the bond performance and no loss in strength was observed. The scatter in the data was within the variability of the baseline non contaminated set. However, A3 contaminated specimens exhibited a significant loss in the fracture toughness with a $\mathrm{G}_{\mathrm{IC}}$ value of $0.57 \mathrm{~kJ} / \mathrm{m}^{2}$, reducing it by $31.3 \%$ when compared to the non-contaminated set. This is attributed to the fact that amount of contaminant deposited via A3 is largely distributed than A1.

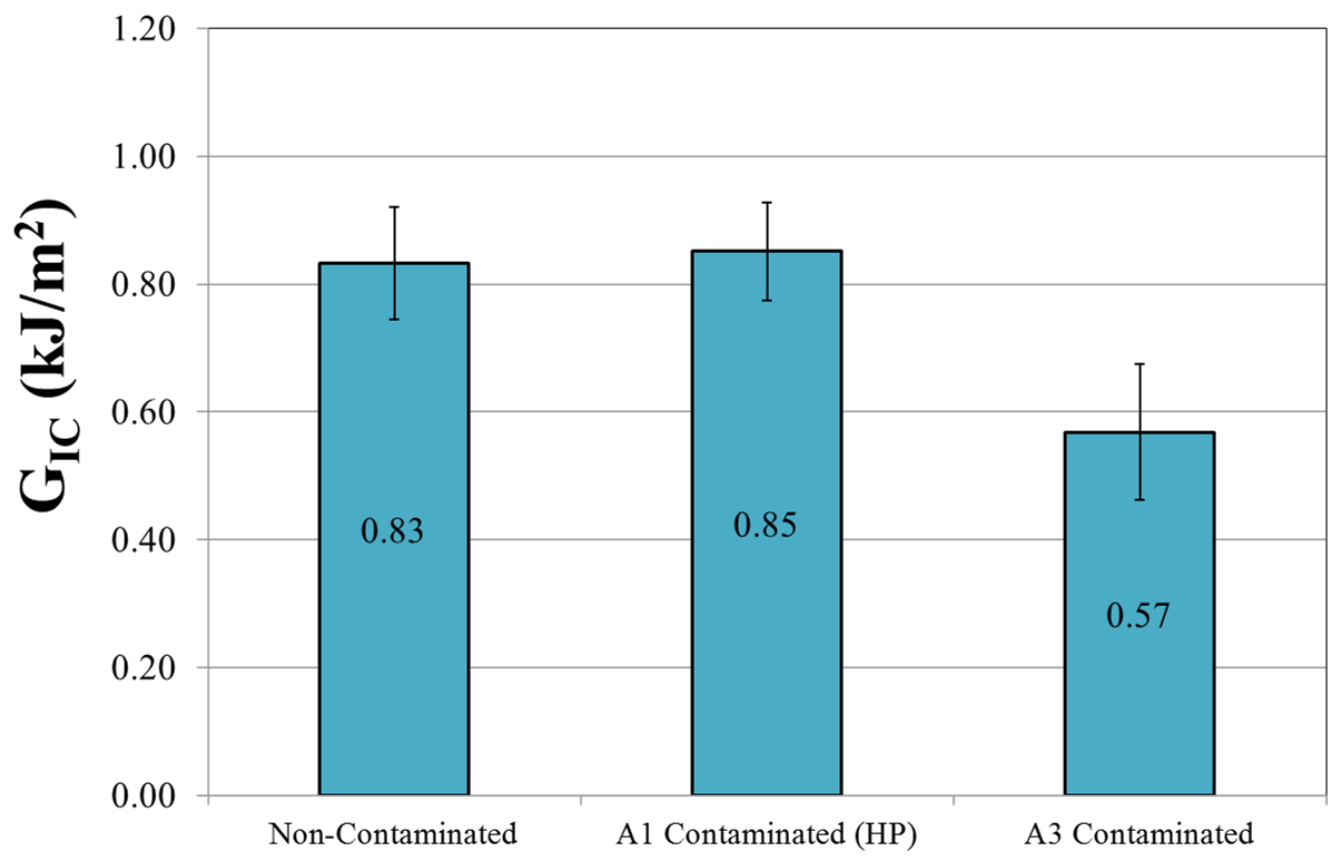

Figure 6.8: Non-Contaminated and Contaminated specimen subjected to mechanical fatigue.

\subsubsection{Combined Environmental Aging and Fatigue}

As part of this study, non-contaminated and contaminated specimens were also subjected environmental exposure and mechanical loading simultaneously. The fatigue loading frequency 
was the same as the fatigue alone conditioning and allowed for the cycles to reach the desired limit in 2 months. After conditioning, the specimens were characterized for their mechanical strength by determining the fracture toughness. The fracture toughness, $\mathrm{G}_{\text {IC }}$ values, for the combined environmental aging and fatiguing for non-contaminated and contaminated specimens are shown in Figure 6.9. For the combined aging, contaminated A1 and A3 specimen sets had a significant reduction in fracture toughness with $\mathrm{G}_{\mathrm{IC}}$ values of $0.70 \mathrm{~kJ} / \mathrm{m}^{2}$ and $0.72 \mathrm{~kJ} / \mathrm{m}^{2}$ (approximately 30\%).This data set is a combination of two aggravating factor i.e. environmental exposure and stressing the bondline via fatiguing; the fracture toughness response is significant in this case affecting the bonded joint. Contamination in addition to the durability testing showed significant adverse effects on the bond performance.

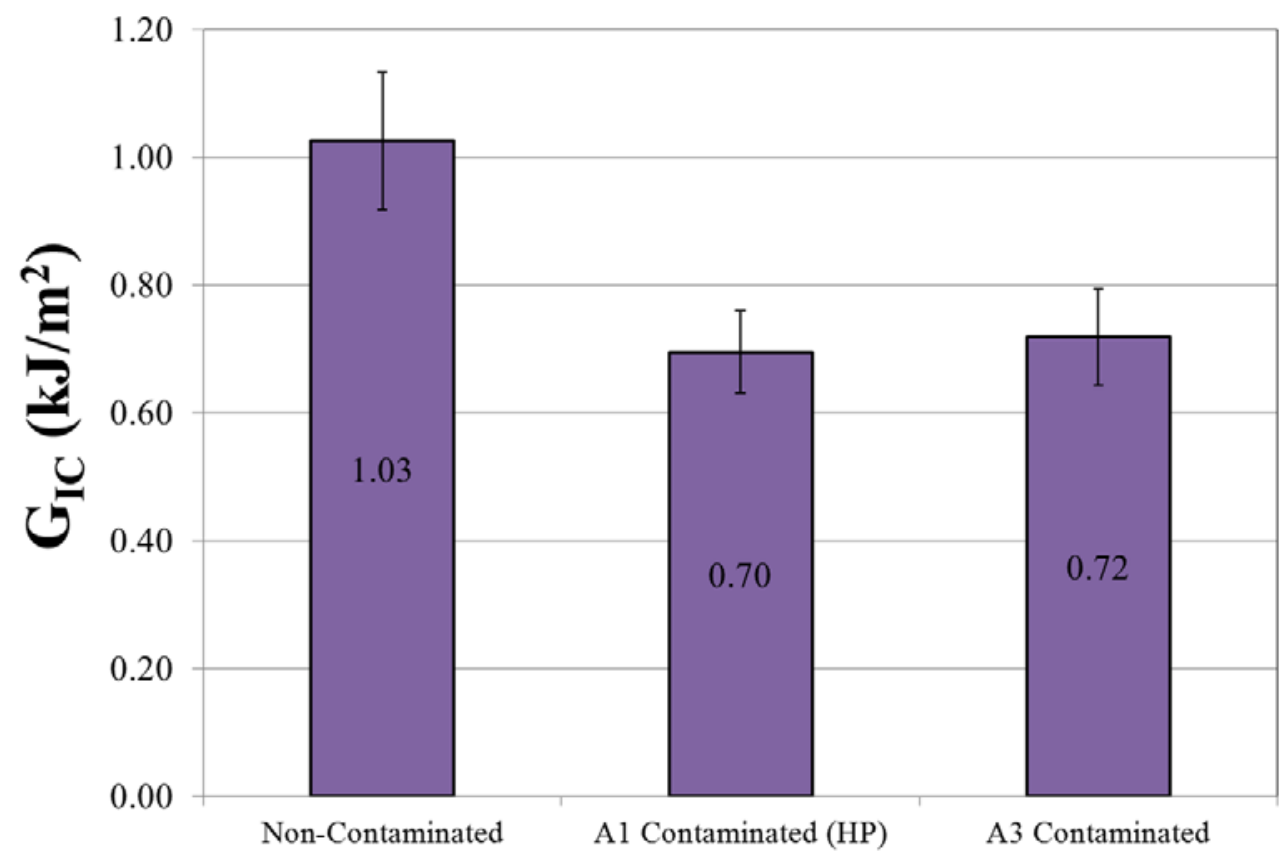

Figure 6.9: Non-Contaminated and Contaminated specimen subjected to combined loading (environmental exposure and mechanical fatigue simultaneously).

Some general conclusions are worth noting here. Although, the specimens are subjected to harsh environmental conditions, the slight tendency to have a constant or rising toughness characteristic is a common trend that was observed by an number of researchers $[17,16,12,13$, 
15]. This behavior was attributed to the plasticization of the adhesive layer upon aging. Additionally Joshi et al. [17] reported that epoxies tend to show a larger scatter during the initial stages of ageing. These results also demonstrate variability with our epoxy based adhesive. Longer aging times for some specimen sets show decreasing trends and will be discussed in later sections. Additional information will also be provided for the modes of failure in the following sections.

\subsubsection{Assessing the Mode of Failure for Conditioned Specimens}

In order to understand the failure modes of the aged specimens, all fracture surfaces were evaluated where the first $5 \mathrm{~cm}$ of the bonded joint was investigated as per ASTMD5528 standards.

\subsubsection{Non-Contaminated specimens subjected to Durability}

The failure modes for non-contaminated specimens that were subjected to three aging conditions are shown in Figure 6.10. For the baseline specimens shown in Figure 6.10a, no pure cohesion failures were observed with specimens having adhesive on both sides of the adherends. Small sections of interlaminar failures were observed at the edges of each specimens. Fracture surfaces for the specimens that were aged in the environmental chamber for 2 months are shown in Figure 6.10b. For this set, we observed a larger amount of interlaminar failures emanating form the edges. This can be attributed to the cutting of the specimens and the creation of pathways for water ingression emanating from the edges. The interlaminar failure dominating at the edges is clear evidence that the water ingressed into the laminate plies through the edges of the coupon. Due to the fact that exposure time is not sufficient for the water to ingress into the center of the specimen (midplane) and that the coupons are unstressed, there was no reduction in the fracture toughness. 


\section{Non Contaminated}

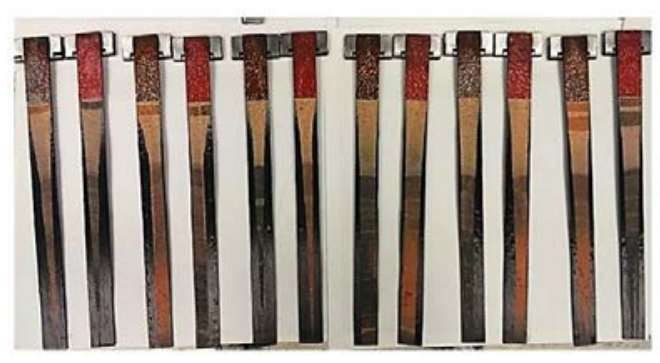

a) Baseline

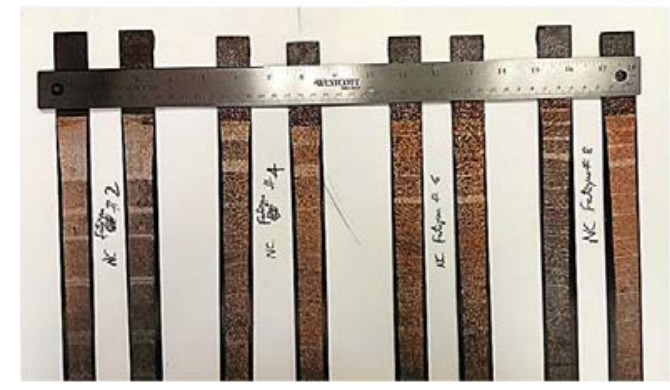

c) Fatigue

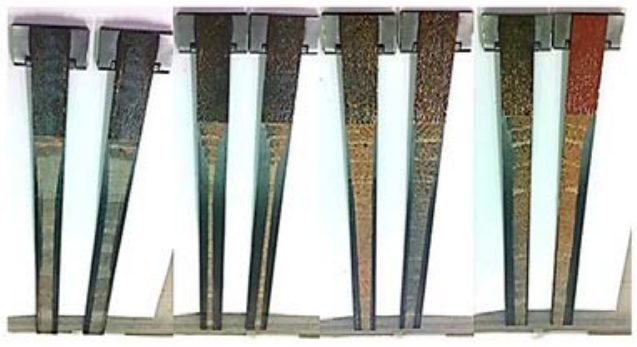

b) Environmentally Exposed
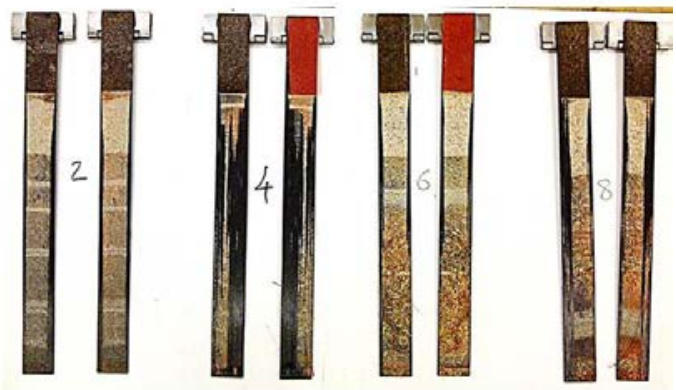

d) Combined Fatigue and Environmental Exposure

Figure 6.10: Comparison of mode of failures for non-contaminated specimens that underwent durability route.

For the specimens that were aged via fatigue in ambient air for 2 months, no crack propagation was observed within the specimens during the fatigue test. It should be noted that a precrack is present for all the specimens and and are been stressed. The coupons sustained the fatigue cycle limit succesfully. $G_{1 C}$ values increased by $~ 10 \%$ for fatigue specimens when compared to their baseline set. Visual observation shows primarily cohesion cohesion failure in all the specimens (Figure 6.10c). The large cohesive area dominance is attributed to increased fracture toughness when fatigued in ambient air for all the specimens. Additionally, specimens that were subject to fatigue, induce strain within the bondline due to compression and tension. The entanglement that is associated with the polymeric chains will try to orient themselves when under compression and tension. This orientation (increase in entanglement) within the chain structure causes more plastic rearrangements as the chain stretches i.e. under compression and tension 
leading to strain hardening mechanism. This is verified by the crack tip opening displacements (CTOD) values for all the specimens that underwent durability as shown in Figure 6.11. For the specimens that are subjected to fatigue and environmental exposure simultaneously, cohesion failures are observed with minor interlaminar failure. The fatiguing induces strain hardening while the moisture is plasticizing the adhesive. This plasticization will further cause the polymeric chain rearrangement to intensify and hence the resistance to deformation within the adhesive increases. The material bears a capacity to carry higher loads in a small contact area within the adhesive/bondline causing strain hardening [18]. This is further verified by the CTOD values as shown in Figure 6.11 where it is the largest for combined fatigue loaded and environmental exposed specimens.

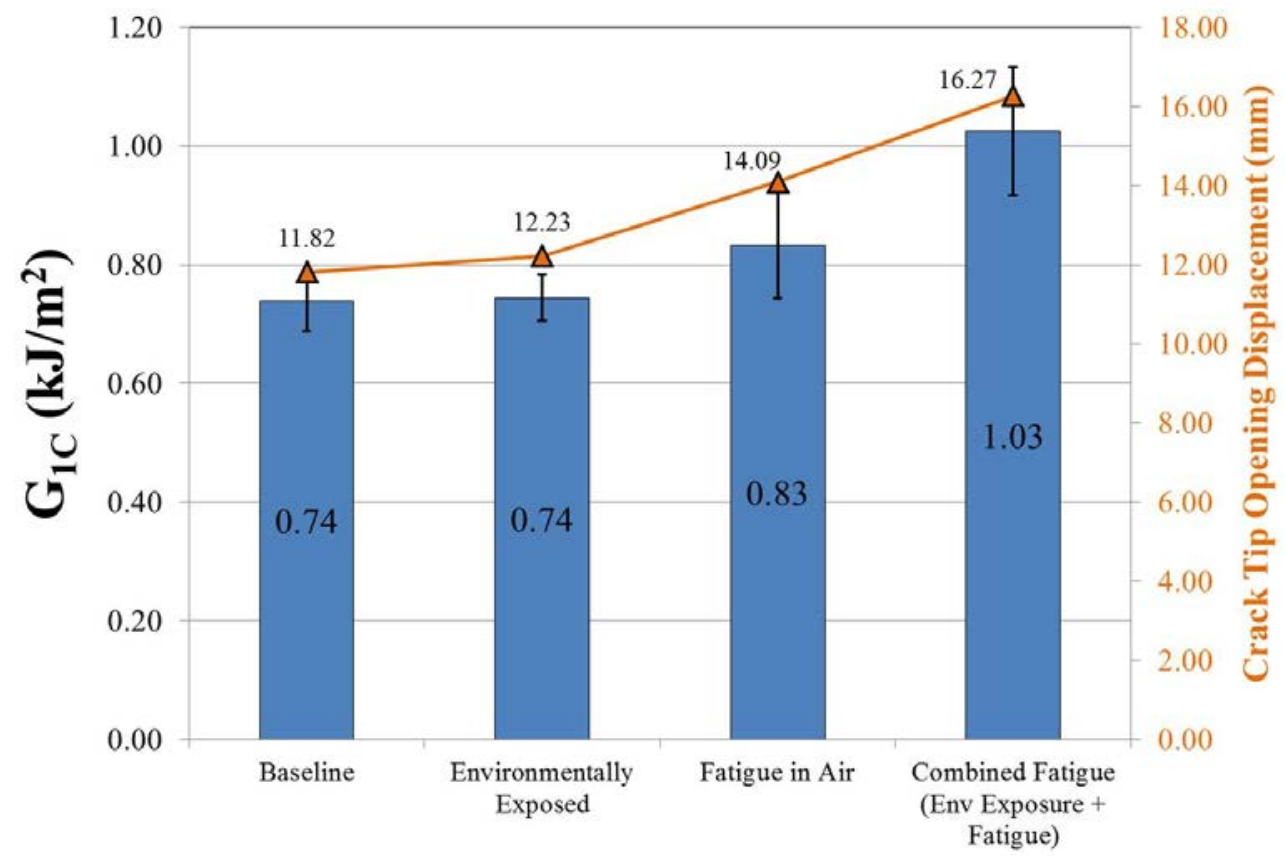

Figure 6.11: Effect of Durability on CTOD for non-contaminated specimens

\subsubsection{Contaminated specimens subjected to Durability}

The failure modes for both contaminated sets subjected to aging were investigated to understand their fracture mechanisms. For both contaminated sets, the dominant failure mode was 
a pure mixed mode with adhesion failures patterned within cohesion failures. Pure adhesion failures were not observed.

\subsubsection{1. $\quad$ A1 contaminated specimens subjected to durability}

For the specimens contaminated with the A1 stamp, pure cohesion failures were observed at the site where no contamination was present. Adhesion failures were observed at the contaminated sites. This showed that the contaminant is highly effective in release at the site and does not allow bonding with the adherend. With the obtained fracture pattern, it can be understood that if a joint is loaded, due to the distribution of ordered contaminant sites, the joint will always fail at the contaminated site but overloads the adhesive at the non-contaminated site. This will typically generate a mixed mode failure pattern which is seen in Figure 6.12. Very marginal interlaminar failure was observed unlike the baseline specimens likely due to stress re-distribution between the localized contaminant sites. In the case of non-contaminated set (see section 6.3.2.1), the fracture always progressed towards the weakest link which was the edge of the specimen due to cutting effects and hence an interlaminar failure was observed. For the contaminated sets, the weakest link is the contaminated site where the fracture primarily progresses within the site and later progresses towards the edge of the specimen. The adhesive is constantly loaded and unloaded in this context where stress re-distribution occurs and hence very marginal interlaminar failures are observed in all the A1 contaminated specimens. All the specimens subjected to durability exhibited a similar behavior and identical failure modes were observed. The CTOD values were plotted to explain the increase in fracture toughness as shown in Figure 6.13. 


\section{A1 Contaminated}

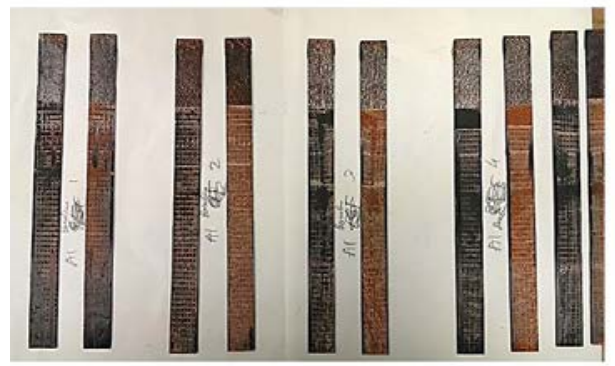

a) Baseline

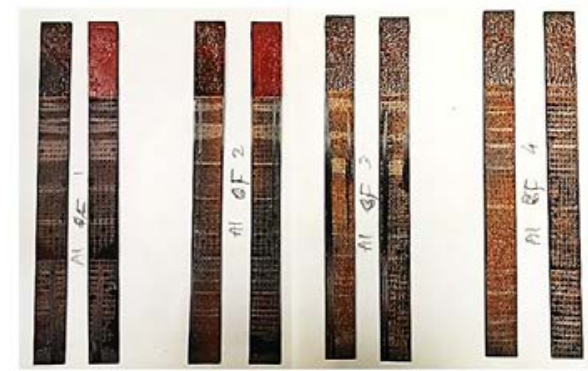

c) Fatigue

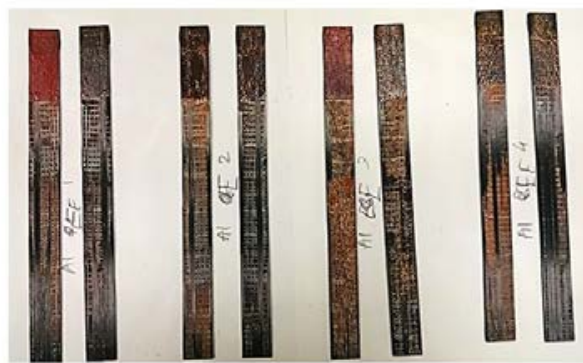

b) Environmentally Exposed

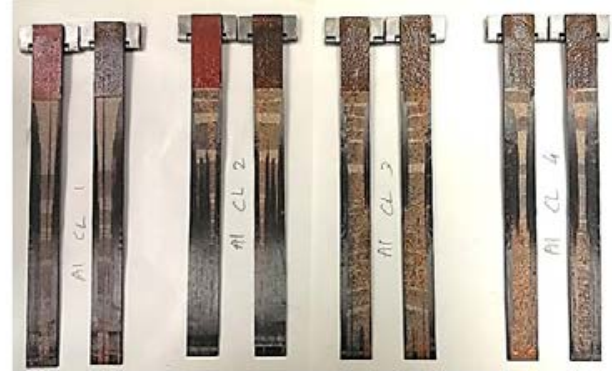

d) Combined Fatigue and Environmental Exposure

Figure 6.12: Comparison of mode of failures for A1 contaminated specimens that are subjected to durability.

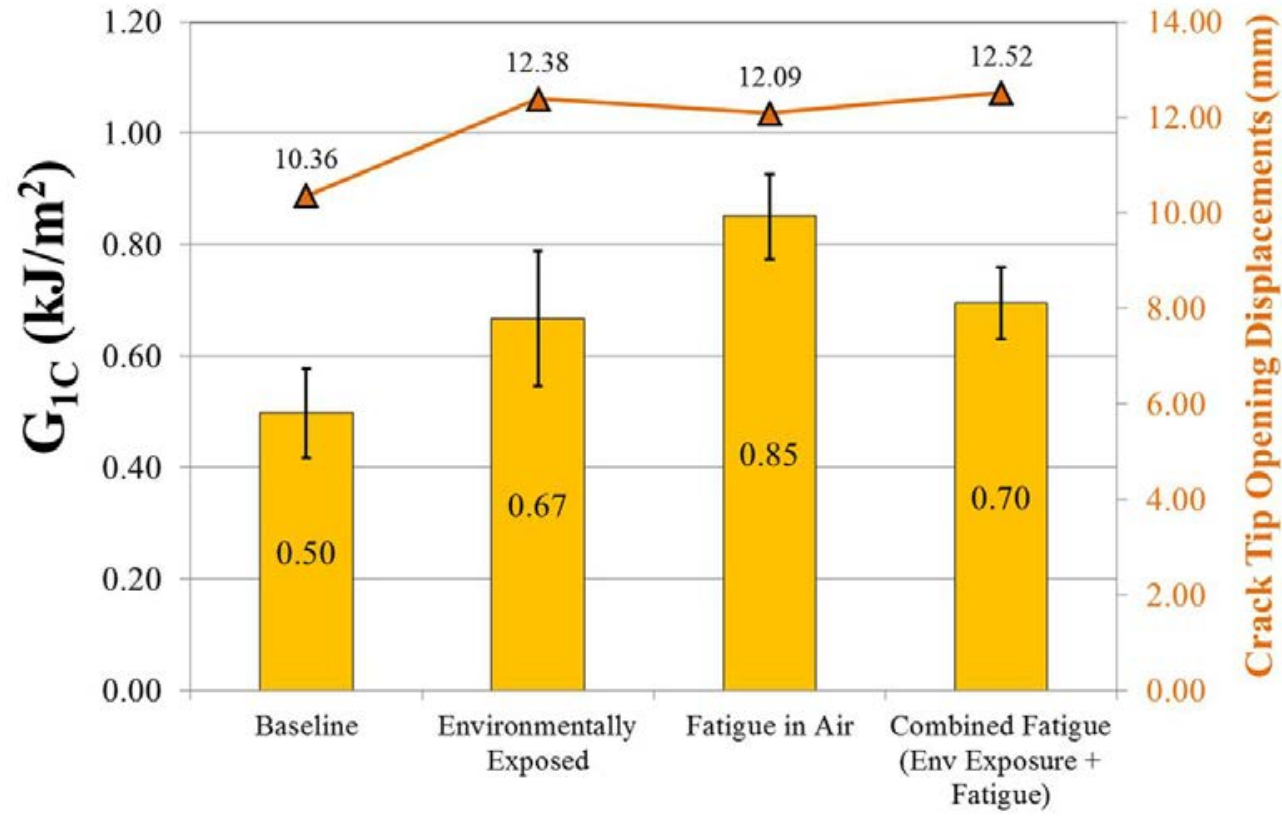

Figure 6.13: Effect of Durability on CTOD for A1contaminated specimens 
In the case of environmentally exposed specimens, the increase in the fracture toughness value is attributed to the plasticization of the adhesive which is correlated with the corresponding increase in the CTOD value. It should to be noted that the environmentally exposed specimens are unstressed and hence strain hardening does not occur. However, the plastic deformation at the crack tip is increased with a CTOD value $(12.38 \mathrm{~mm})$ which is a $10.8 \%$ increase over the baseline set. For the fatigue specimens, the increase in fracture toughness is primarily attributed to area of the contaminant covered which was marginal i.e. large cohesive failures were seen on both sides of the specimens with minimal adhesive failure regions indicating that the contaminant distribution was minimal. When subjected to fatigue aging, the specimens exhibited an identical behavior as of the non-contaminated specimens where strain hardening occurred and increased the fracture toughness. A1 contaminated specimens upon subjected to combined loading exhibited a different mode of failure where mixed mode pattern was not visible. This was attributed to the manufacturing defect where air channels were clearly visible at the bondline. However, fatiguing and environmental exposure affected the CTOD value and was found to be the highest where the induced strain showed no effect with the presence of contaminant.

\subsubsection{2. $\quad$ A3 contaminated specimens subjected to durability}

The A3 contaminated specimens exhibited pure cohesion failures at the sites where no contamination was present and adhesion failures at the contaminated sites. The A3 mode of failure replicates the pattern created by the stamp. Due to the low viscosity of the contaminant, maximum care was taken to avoid the spreading by not applying any gravimetric pressure on the stamp and ensuring that the contaminant pattern is just transferred. A3 stamp procedure is an additional level of contamination with increasing contamination size, the failure mode can be easily seen with adhesion failures surrounded by cohesion failures. The redistribution of load with the largely nonbonded contaminant area is pronounced with this level of contamination. These typically generate a mixed mode failure pattern which is seen in Figure 6.14. 


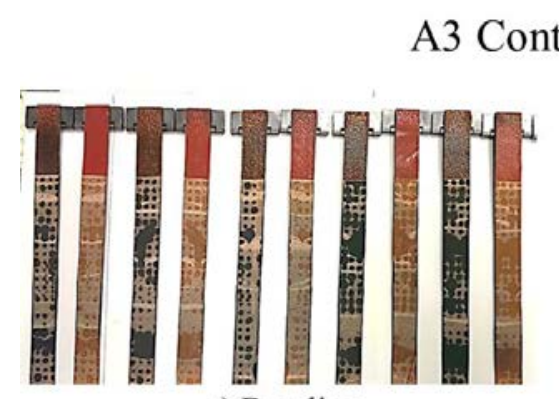

a) Baseline

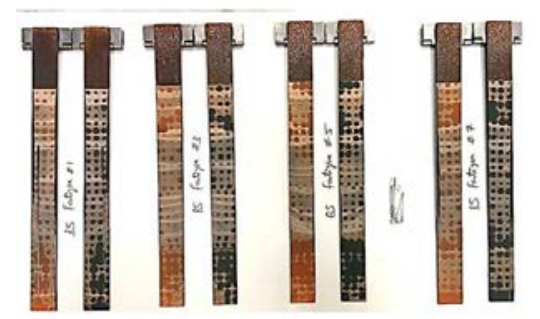

c) Fatigue

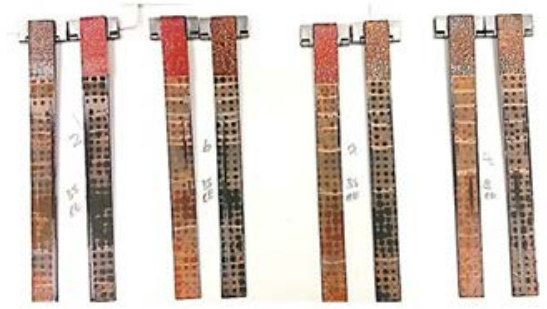

b) Environmentally Exposed

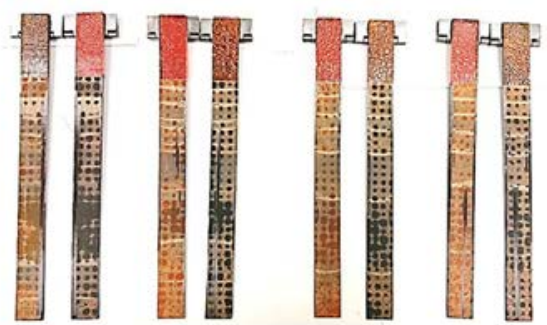

d) Combined Fatigue and Environmental Exposure

Figure 6.14: Comparison of mode of failures for A3 contaminated specimens that are subjected to durability.

In each of the 3 conditioned sets that were contaminated with the A3 stamp, there was no interlaminar failure. This is likely due to the stress re-distribution as explained in section 6.3.2.2.1. Additionally crack bluntness that is caused due to the large dotted pattern will be a factor that influences the fracture toughness. Plasticization again corroborates with the CTOD values i.e. upon environmental ageing, the crack tip plasticity will increase causing the CTOD values to increase. This plasticizing ability is expected due to the unstressed condition of specimen, the localized plasticity of adhesive dominates upon moisture ingression. With fatiguing, although the adhesive strain hardens, the CTOD values will tend to decrease due to the large size of the contaminant influencing the crack opening and is clearly seen in the failure modes as well as in Figure 6.15. In the case of combined loading, the CTOD values tend to decrease further due to the combination effects of strain hardening, moisture ingression and contamination. The CTOD is never large where there are intermediate voids even after strain hardening and plasticization unlike with the non- 
contaminated specimens where CTOD values increased with combined loading due to absence of voids i.e contamination.

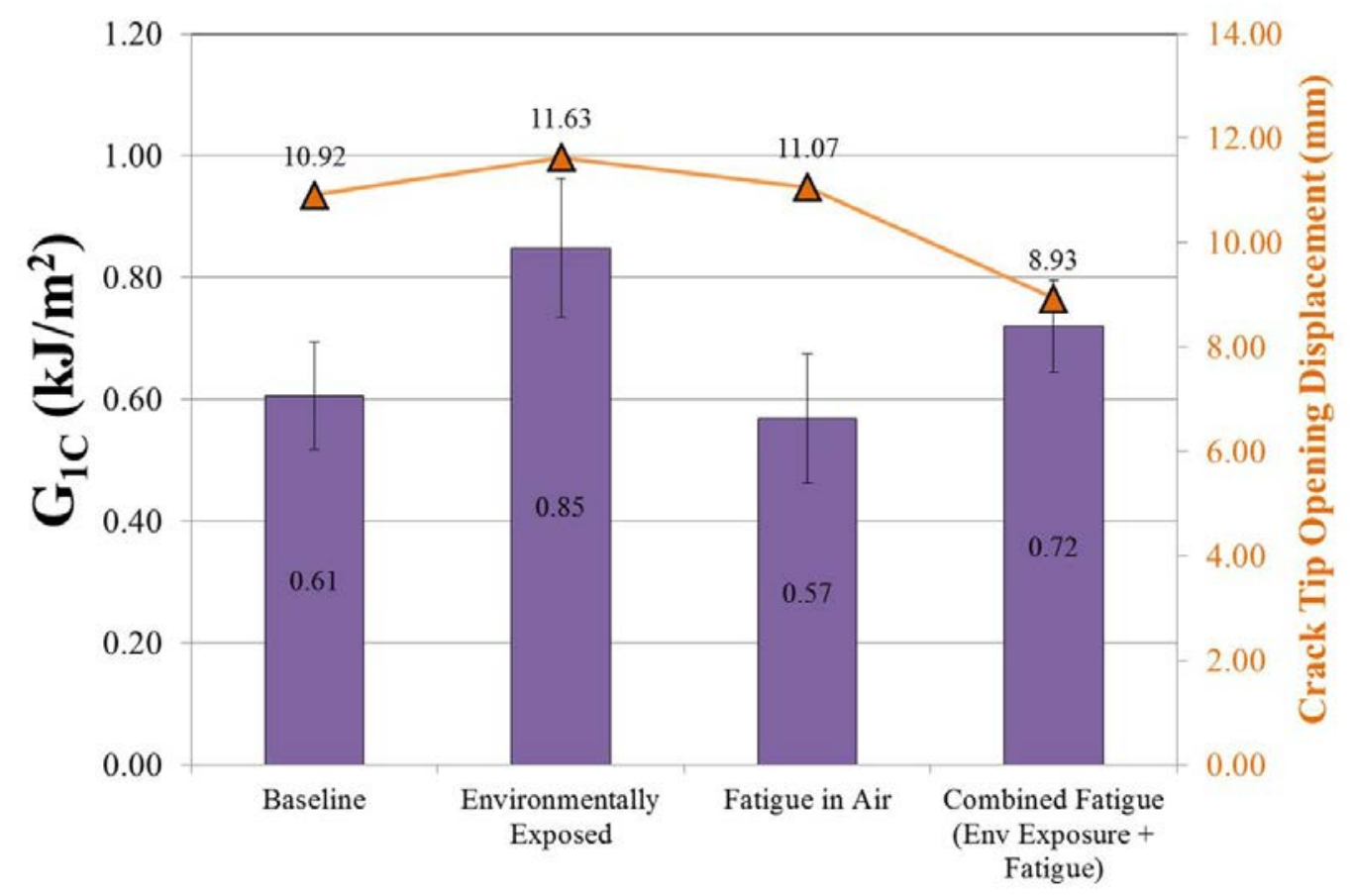

Figure 6.15: Effect of Durability on CTOD for A1contaminated specimens

\subsubsection{Moisture Absorption Study Results}

Epoxy polymers can absorb moisture when subjected/exposed to humid environments.

This process occurs through diffusion and the moisture uptake percentage can be plotted as a function of time to determine saturation levels. For this study, moisture uptake information was obtained for specimens that were laminates only and bonded laminates over a period of 78 weeks. Figure 6.16 shows a plot of the moisture uptake data for both the specimen sets. Asymptotic absorption behavior is observed. The percentage uptake increased rapidly during the first few weeks but slowed in the following weeks. The composite laminate absorbed $0.848 \%$ of moisture and the adhesive by itself $0.104 \%$ when compared to their initial weights prior to exposure. This 
aids in understanding the time required for the specimens to saturate and the \% saturation can dictate the effect on the bond strength.

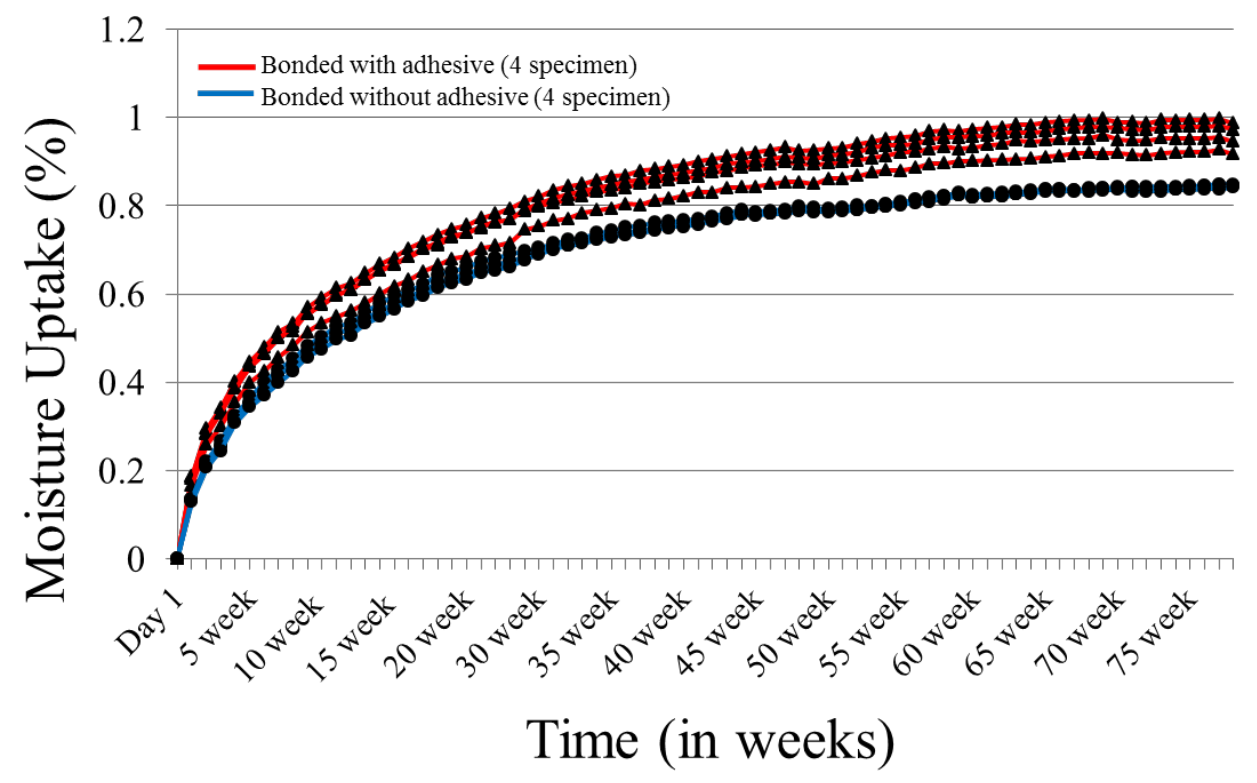

Figure 6.16: Moisture uptake \% plot for bonded composite laminate and laminate only.

\subsubsection{Fracture Toughness evaluations for Long Exposure specimens}

After the bonded specimens were exposed to 80 weeks of elevated temperatures and humidity, they were removed to evaluate their change in fracture toughness. DCB Results from long term exposure samples are shown in Figure 6.17 where they are compared with samples exposed for 8 weeks. As discussed earlier in section 6.3.2.1 (see Figure 6.11), 8 weeks of exposure did not affect the specimens fracture toughness. The specimens that are exposed for longer duration (80 weeks) show a significant degradation in the bond strength by $~ 19.65 \%$ when compared to the baseline set. The loss in the strength is attributed to the weakening of the interface at the bondline via diffusion of water molecules into the specimens which can be seen in Figure 6.17. The moisture content of baseline specimens start at zero increasing to $0.55 \%$ for bonded specimens and $0.46 \%$ for non-bonded specimens. It can be concluded that $0.5 \%$ of moisture uptake for both ABCJ's and the laminate does not influence their mechanical properties. 
While on the other hand, moisture uptake $\%$ when reached upto $0.944 \%$ exhibited a significant loss in the bond strength of adhesively bonded specimens and the laminate alone with $0.85 \%$ of water uptake. The failure modes of adhesively bonded specimens are shown in Figure 6.18 where a significant interlaminar failure is observed. The adhesive bonds prepared sustained the durability of harsh environmental conditions for 60 days without loss in their strength while those exposed to 480 days suffered a reduction in their bond strengths by $19.65 \%$. The failure mode progressed from cohesive to interlaminar with hygrothermal ageing. It is of prime importance to note that there were no adhesion failures observed for the accelerated ageing procedure adopted to characterize the long term durability of bonded composite joint suggesting that the bonds produced were durable to overcome the interfacial strength between adhesive and adherend.

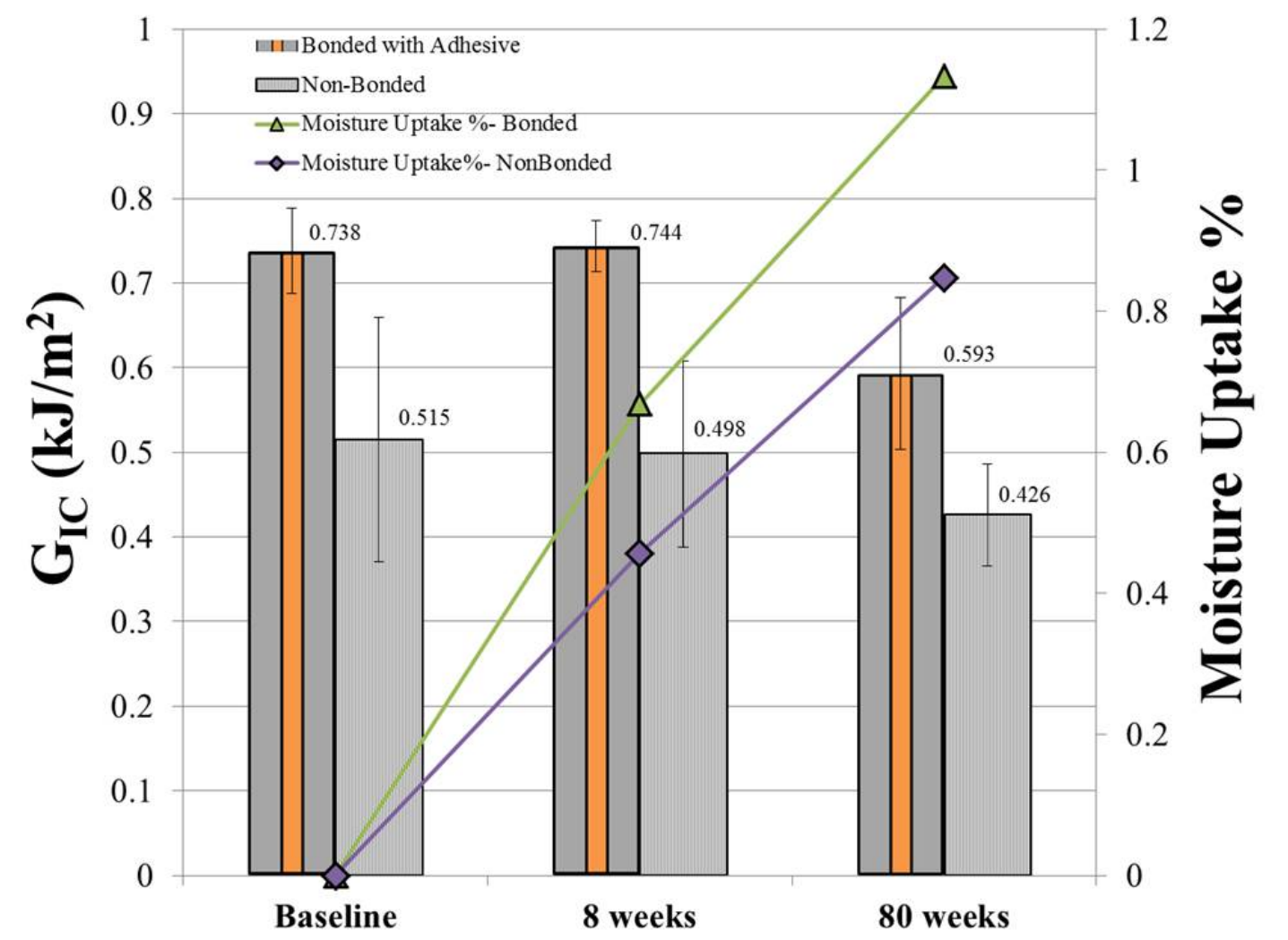

Figure 6.17: Effect of moisture ingression on bond strength of bonded and non-bonded specimens with varying exposure times. 


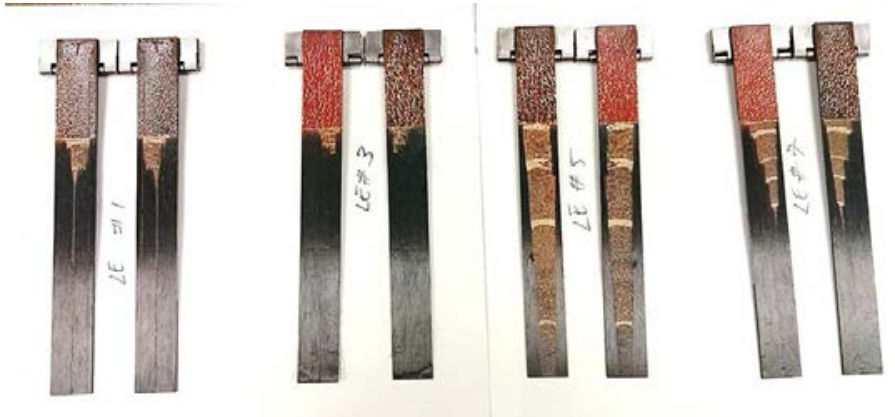

Figure 6.18: Mode of Failure for long exposure specimens bonded with adhesive.

\subsection{Conclusions}

A test procedure was successfully demonstrated in order to evaluate durability of adhesively bonded composite joints. The durability route adopted was environmental conditioning, fatiguing in ambient air and combined loading i.e. fatiguing and environmental exposure simultaneously. This was successfully conducted on pristine specimens and specimens that were manufactured with undesirable bonding conditions. The failure modes were largely cohesive with interlaminar failure at the edges for pristine specimen while the specimens that were contaminated exhibited a pure mixed mode failure i.e. an adhesive failure surrounded by a cohesive failure. The specimens that were showing an increased fracture toughness was related to plasticization that corroborated with CTOD measurements. The specimens that were fatigued experienced a higher toughness values attributing it to the strain hardening that were in agreement with CTOD displacement measurements. Contaminated specimens showed a lower CTOD values when compared to pristine specimen due to the presence of flaws within the adhesive-adherend interface. Interlaminar edges which are attributed to the stresses generated in the laminate plies needs further refinement in the cutting procedure that can minimize the edge effects. Moisture uptake levels upto $0.5 \%$ did not have any deleterious effect on the bond performance which can serve as a preliminary durability dataset for ABCJ's utilized. While a 0.9\% moisture uptake had exhibited a 20\% reduction in the bond performance. No adhesion failures were observed in any of the bonded composite joints. 


\subsection{References}

[1] W. Broughton and R. Mera, "Review of Durability Test Methods and Standards fro Assessing Long Term Performance of Adhesive Joints," National Physical Laboratory, Middlesex, UK, May 1997.

[2] J. Crank, Mathematics of Diffusion, 2nd Edition, Oxford : University Press, 1975.

[3] J. Crank and G. Park, Diffusion in Polymers, London: Academic Press, 1968.

[4] I. Ashcroft, D. Hughes and S. Shaw, "Adhesive Bonding of Fibre Reinforced Polymer Composite Materials," Assembly Automation, vol. 20, no. 2, pp. 150-161, 2000.

[5] I. Ashcroft, R. Digby and S. Shaw, "Accelerated ageing and life prediction of adhesively bonded joints," in EURADH 4th European Conference on Adhesion and WCARP 1, 1st World COngress on Adhesion and related phenomenon., Garmish-Partenkirchen, 1998.

[6] G. Doyle and R. A. Pethrick, "Environmental effects on the ageing of epoxy adhesive joints," International Journal of Adhesion \& Adhesives, vol. 29, pp. 77-90, 2009.

[7] S. Orman and C. Kerr, Aspects of Adhesion, London: University of London Press, 1971.

[8] A. Crocombe and A. Kinloch, "Review of Adhesive Bond Failure Criteria," MTS Adhesives Project 2, August 1994.

[9] S. Zainuddin, M. Hosur, Y. Zhou, A. Kumar and S. Jeelani, "Durability studies of montmorillonite clay filled epoxy composites under different environmental conditions," Material Science and Engineering A, vol. 507, pp. 117-123, 2009.

[10] V. L. Saponara, "Environmental and chemical degradation of carbon/epoxy and structural adhesive for aerospace applications: Fickian and anomalous diffusion, Arrhenius kinetics," Composite Structures, vol. 93, pp. 2180-2195, 2011.

[11] M. Bowditch, "The durability of adhesive joints in the presence of water," International Journal of Adhesion and Adhesives, vol. 16, pp. 73-79, 1996.

[12] R. Selzer and K. Friedrich, "Influence of water up-take on interlaminar fracture properties of carbon fibre-reinforced polymer composites," Journal of Materials Science, vol. 30, pp. 334-338, 1995.

[13] R. Selzer and K. Friedrich, "Mechanical properties and failure behavior of carbon fibrereinforced polymer composites under the influence of moisture," Composites: Part A, vol. 28A, pp. 595-604, 1997.

[14] J. Lucas, J. Zhou and Y. Wai, "Micromechanics Analysis of Fibre Fragmentation Test," in Proceedings of ICCM 9 in COMPOSITES: Properties and Applications, Madrid, 1993.

[15] S. Pantelakis and K. Tserpes, "Adhesive bonding of composite aircraft structures: Challenges and recent developments," Science China Physics, Mechanics and Astronomy, vol. 57, no. 1, pp. 2-11, Jan 2014. 
[16] C. V. Katsiropoulos, A. Chamos, K. Tserpes and S. G. Pantelakis, "Fracture toughness and shear behavior of composite bonded joints based on a novel aerospace adhesive," Composites: Part B, vol. 43, no. 2, pp. 240-248, 2012.

[17] S. B. Joshi, T. F. Gray, W. M. Banks, D. Hayward, R. Gilmore, L. W. Yates and R. A. Pethrick, "Environmental Ageing of Adhesively Bonded Joints. II. Mechanical Studies," The Journal of Adhesion, vol. 62, pp. 317-335, 1997.

[18] R. S. Hoy and M. O. Robbins, "Strain Hardening of Polymer Glasses: Effect of Entanglement Density, Temperature and Rate," Journal of Polymer Science Part B: Polymer Physics, vol. 44, no. 24, pp. 3487-3500, Nov 2006.

[19] N. Brack and A. Rider, "The influence of mechanical and chemical treatments on the environmental resistance of epoxy adhesive bonds to titanium," International Journal of Adhesion and Adhesives, vol. 48, pp. 20-27, 2014. 


\section{PREDICTION APPROACH}

This chapter describes a fracture mechanistic based model that is fully utilized to predict the fracture toughness of ABCJ's containing undesirable bonding conditions. Undesirable bonding conditions were created using the stamp approach as described in Chapter 3. Fracture toughness was predicted based on the model criteria and assumptions pertaining to adhesive material that were comparably close. The fracture toughness values evaluated from the model is compared with the experimental values.

\subsection{Development of Predictive Model Criteria}

The conventional methods for prediction of a fracture in any material utilizes two basic approaches i.e. 1) Stress or Strain based approach where a material reaches a critical value and failure occurs or 2) Stress intensity of the existing cracks or flaw possessing a stress intensity factor associated with the crack/flaw reaching a critical value [1]. Various equation intensive approaches have been constructed as predictive models that often result in a large number of undetermined parameters. This can potentially limit the predictive power and complicate its comparison with experimental data. Here a simplistic fracture model is discussed that demonstrated how stress intensity factors can be utilized in fracture studies of ABCJ's.

Evolution of microcracks can coalesce to form a macro crack and catastrophically fail in load bearing structures. Simulating these microcracks and predicting the overall mechanical property of a structure can be very time consuming. Simulations at microscale and their integration at a macroscale can be a complex task for a computer. To address this problem, the representative volume element (RVE) approach was followed for the prediction of fracture toughness. Fang et.al [2] defined the RVE theory as the global length scale in a global coordinate system is proportional to the local length scale of the unit cell in the local coordinate system and is expressed as

$$
Y_{g}=\frac{y_{l}}{\epsilon}
$$


where $Y_{g}$ is the length scale in global coordinate system;

$y_{l}$ is the length scale in local coordinate system;

$\epsilon$ is the scaling parameter that is always equal to the ratio of unit cell size to the macroscopic region of the global coordinate system in which it belongs.

This theory served as motivation to construct an RVE model on ABCJ's as mentioned below. Considering a circular crack flaw at the interface of a material having radius, $\mathrm{a}_{\mathrm{f}}$ and height, $\mathrm{d}$ at the adhesive-adherend interface. For convenience, the flaw is assumed to be small when compared to the width of the plate. Figure 7.1 shows the schematic of a true representation of the contaminated site at the interface between an adherend and adhesive in an ABCJ.

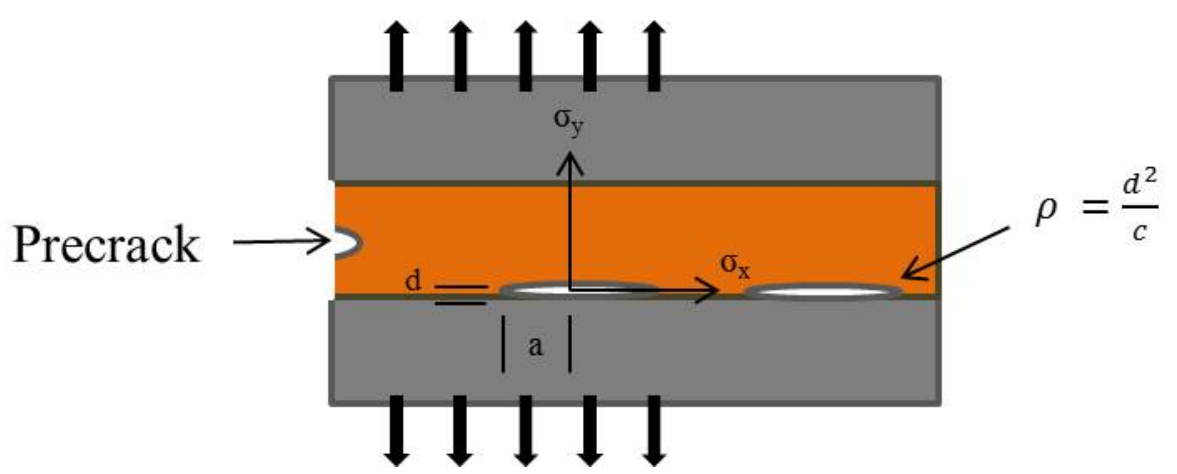

Figure 7.1: Schematic of the true representation of the contamination link at the bonded interface.

A remotely applied uniform stress, $\mathrm{S}$ is applied on the adherends. The most influential effect on the flaw is the stress field $\sigma_{\mathrm{y}}$ acting parallel to $\mathrm{S}$. The top view of the schematic can be constructed as RVE model as shown in Figure 7.2. For the prediction approach, an RVE unit cell was considered which is shown in red. In our experimental methodology, the contaminant is at the interface between the adhesive and adherend. In this model, an identical criteria is assumed by considering the flaw to be present at the interface. The grey region represents adherend and the localized contaminated sites with a diameter of $2 \mathrm{a}$ and the orange represents the adhesive. As 
explained in Chapter 5 (see section 5.4.3-Figure 5.10), the modes of failure occur in a mixed mode i.e. areas of localized adhesion surrounded by area of cohesion. This served as a motivation in considering RVE as a dotted array pattern distributed across the entire width of the specimen. Assuming that the crack propagates from top to bottom within the within a unit cell as shown in Figure 7.2, the stress intensity factor, $\mathrm{K}_{\mathrm{c}}$ is given as

$$
K_{c}=\sigma_{y} \sqrt{\pi a}
$$

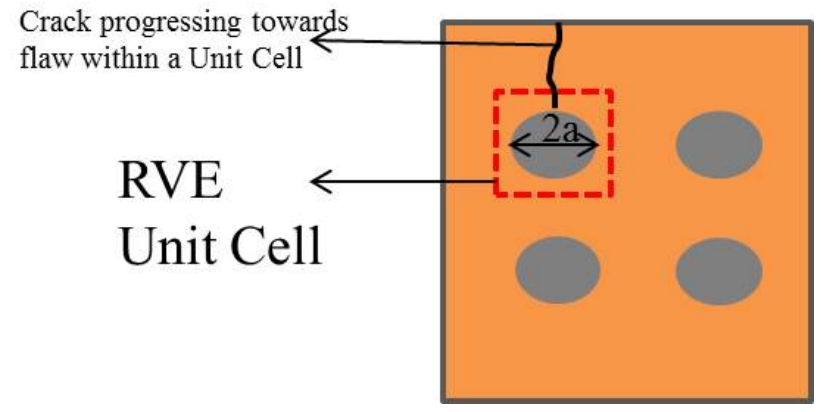

Figure 7.2: RVE Unit cell drawn in red with a local coordinate.

In this case, $\mathrm{K}$ depends on the remotely applied stress $\sigma_{\mathrm{s}}$ and the circular flaw size, a. The applied stress $\sigma_{\mathrm{s}}$ is the force acting per unit area

$$
\sigma_{s}=\frac{\sigma_{y}}{A}
$$

where the force translates to tensile strength of the adhesive, $\sigma_{y}$ which is unknown and A is the unit cell area, $A_{\text {UnitCell }}$

$$
A * \sigma_{s}=\sigma_{y}
$$

Substituting $\sigma_{y}$ in $K_{c}$,

$$
K_{c}=A * \sigma_{s} \sqrt{\pi a}
$$

As mentioned, Unit cell area, $A_{\text {Unitcell }}$ is the sum of the areas of cohesive element and adhesive element.

$$
A_{\text {Unitcell }}=A_{\text {cohesive }}+A_{\text {adhesive }}
$$


As mentioned in section 5.4.2.1, the effect of area played a crucial role in governing the fracture toughness, GIC of a joint and hence areas were quantified and plotted against GIC’s.

Therefore,

$$
A_{\text {cohesive }}=A_{\text {UnitCell }}-A_{\text {adhesive }}
$$

Hence Kc can be rewritten as

$$
K_{c}=A_{\text {cohesive }} * \sigma_{s} \sqrt{\pi a}
$$

This is the empirical relation between stress intensity factor, $K_{c}$ and cohesive area, $A_{\text {cohesive }}$. A linear relationship should be estimated based on the equation above. The RVE unit cell with the Stress Intensity factor $K_{c}$, is calculated based on various flaw sizes ranging as low as $2 \mathrm{~nm}$ to 6 $\mathrm{mm}$. The unit cell assumed was to be $1 \mathrm{~cm} \mathrm{x} 1 \mathrm{~cm}$ square. Since the tensile strength of the adhesive is unknown, a close comparable range (3 $7 \mathrm{MPa}$ ) was chosen to observe the effect of varying flaw sizes with varying tensile strength on $K_{c}$. The stress intensity factors are then converted to their respective fracture toughness values as follows:

$$
K_{c}=\sqrt{E G_{C}}
$$

where $\mathrm{E}$ is the Young's Modulus and $G_{C}$ is the fracture toughness. The processed equation can now be written as

$$
G_{C}=\frac{K_{C}^{2}}{E}
$$

In general, young's modulus of typical structural adhesives are in the range of 2000-3000 MPa.

\subsection{Results and Discussions}

The dependency of unit cell cohesion area on $K_{c}$ is plotted in Figure 7.3. A linear relationship was observed between the cohesive area and stress intensity factors. With the young's modulus range mentioned above, it would be a good estimate if the values are chosen in the midrange so that a dependency on the trend can be easily estimated. Young’s Modulus was chosen to be $2500 \mathrm{MPa}$ and the dependency of fracture toughness $G_{C}$ on an RVE unit cell cohesive area 
was plotted as seen in Figure 7.4. The $G_{C}$ values thus obtained are compared with the experimental values.

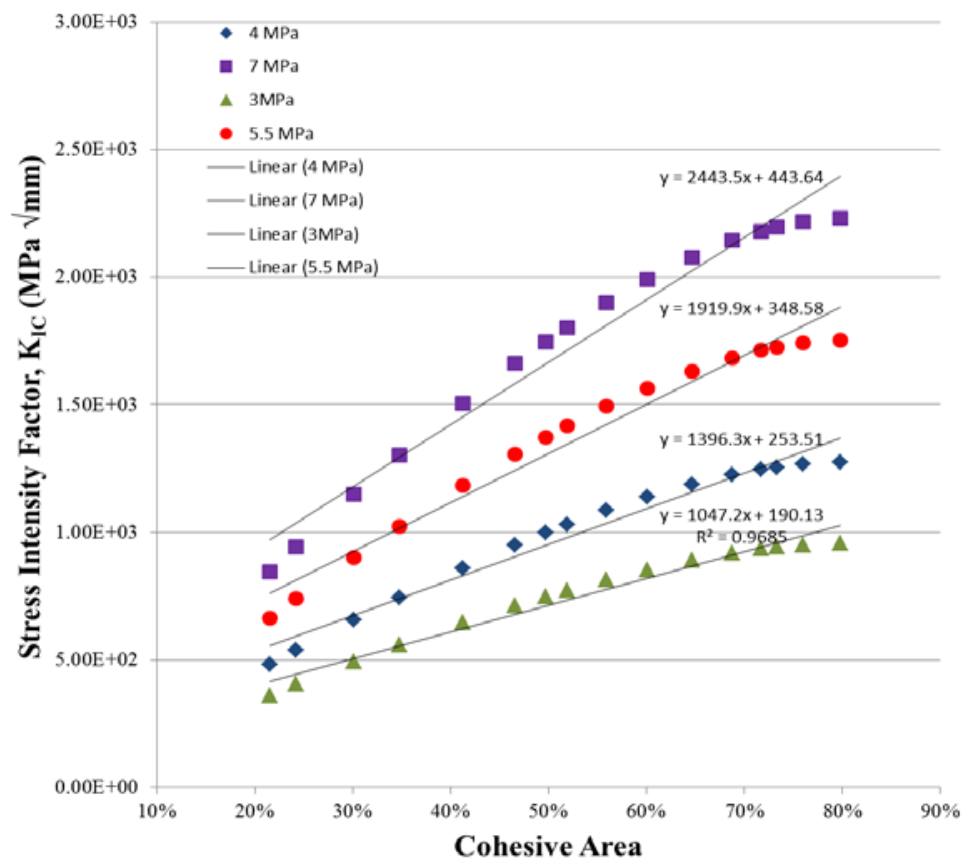

Figure 7.3: Dependency of unit cell cohesion area on $\mathrm{K}_{\mathrm{c}}$

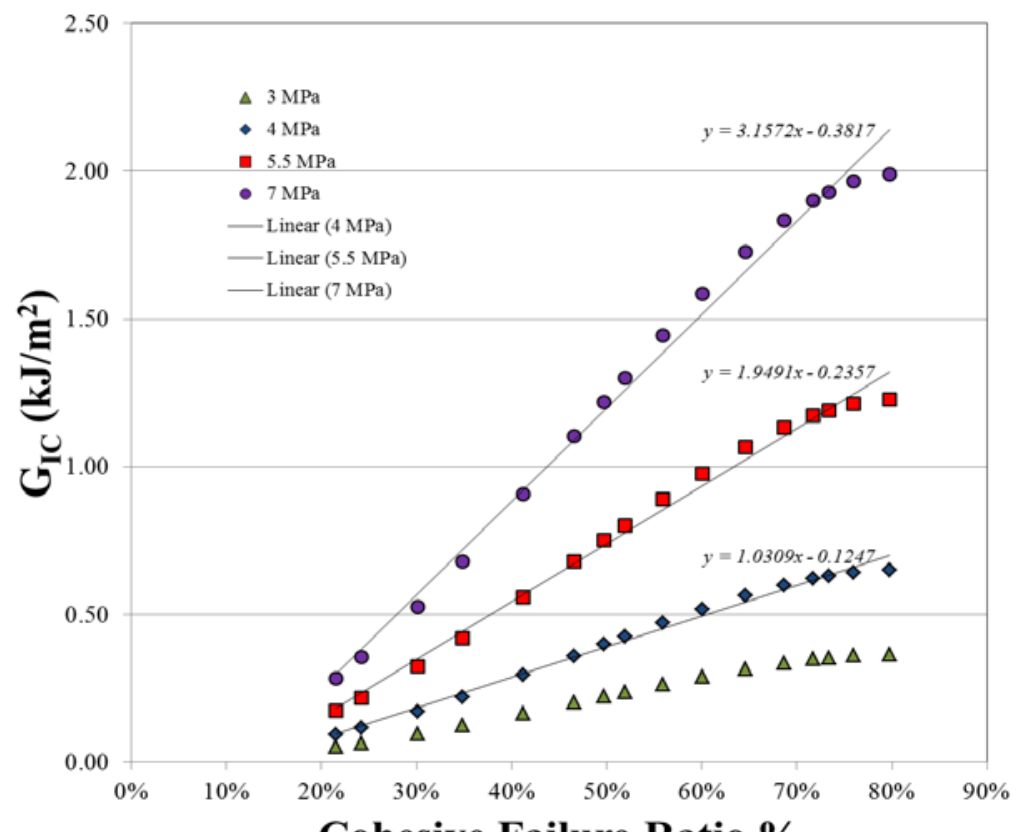

Figure 7.4: $\mathrm{G}_{\mathrm{C}}$ dependency on RVE Unit cell cohesive area 
The predicted fracture toughness $\mathrm{G}_{\mathrm{IC}}$ values increased linearly with cohesive area within an RVE unit cell. As shown in Figure 7.5, the experimental dataset has a slope of 0.0127 and the predicted plot generated a similar slope, with a value of 0.0103 . It is evident that the trend in the predicted plot for RVE unit cell correlates well with the experimental dataset. Having the information of the cohesive area or the area of contamination causing the adhesion failure can assist in predicting the fracture toughness values of the specimens. The predicted results are on a good agreement with the experimental results. The chosen range of flaw sizes that were assumed fits appropriately with fracture toughness values with an average error $\%$ of less than $12.2 \%$ as shown in Figure 7.6. It can also be inferred that for every $10 \%$ increase in the cohesive area ratio, nearly $20 \%$ increase in the $\mathrm{G}_{\text {IC }}$ is affected. This suggests that $10 \%$ increase in contamination could degrade the $\mathrm{G}_{\mathrm{IC}}$ values by nearly $20 \%$.

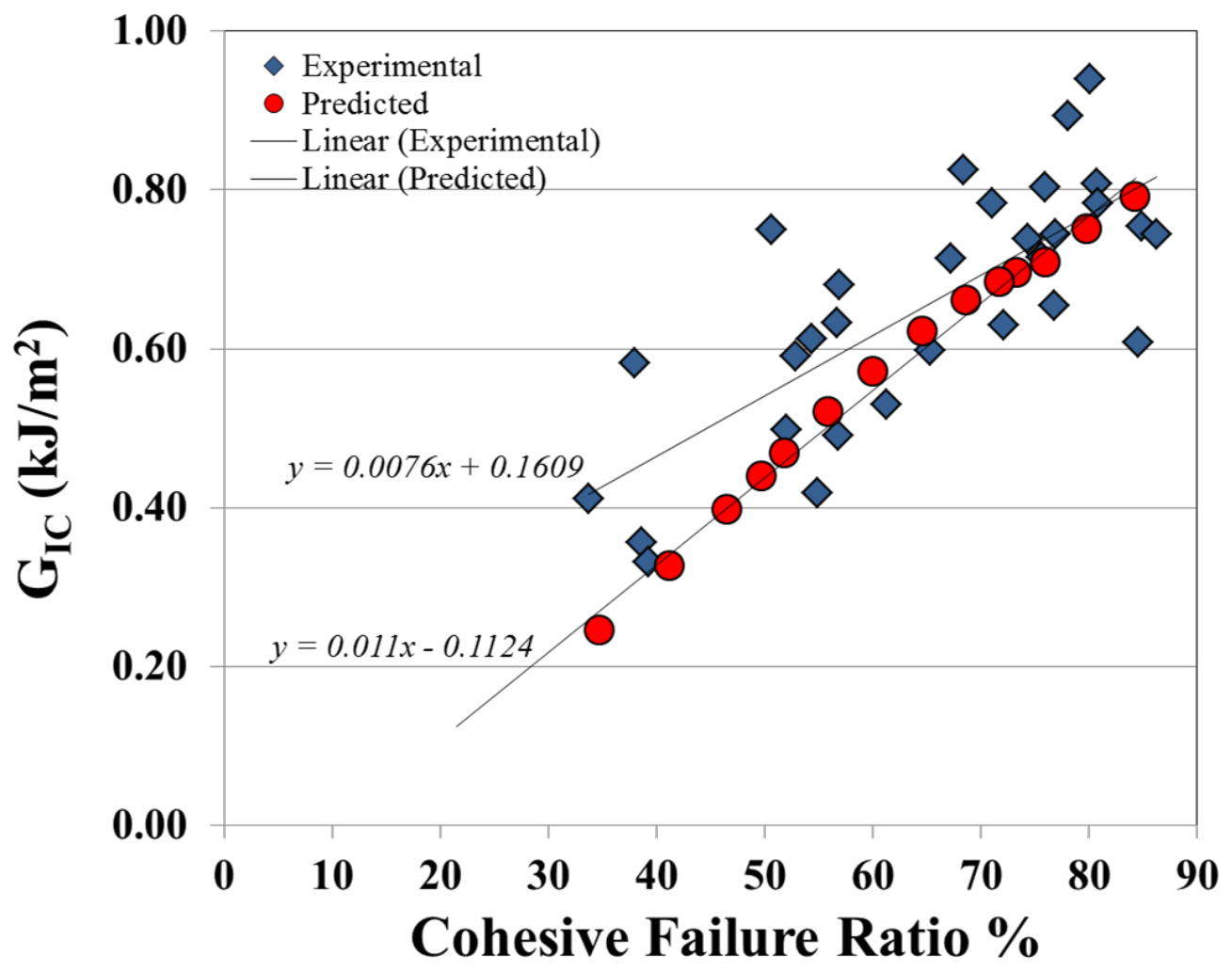

Figure 7.5: $G_{1 C}$ Experimental vs Predicted Slopes 
The experimental and predicted equations are given as,

Experimental, $\mathrm{G}_{\mathrm{IC}}=0.0076\left(\mathrm{~A}_{\mathrm{coh}}\right)+0.1609$

Predicted, $\mathrm{G}_{\mathrm{IC}}=0.011\left(\mathrm{~A}_{\text {coh }}\right)-0.1124$

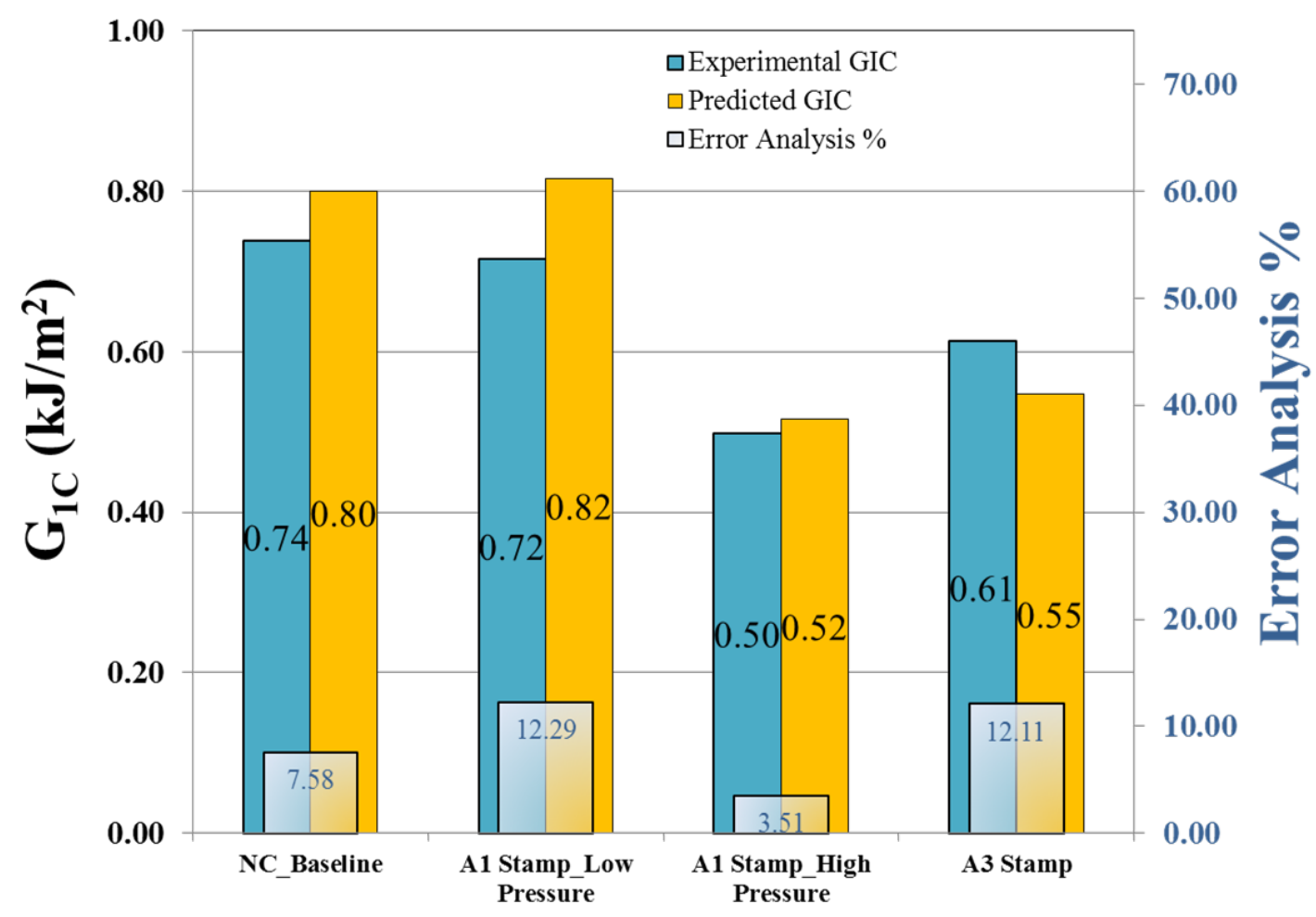

Figure 7.6: Predicted vs Experimental Fracture Toughness plots

\subsection{Conclusions}

The use of RVE unit cell model has been effectively modified to predict the fracture toughness of adhesively bonded composite joints using undesirable bonding conditions. In this study, a theoretical formula was developed for predicting fracture toughness that was compared with the experimental dataset and was found to be in good agreement. The developed model was validated by varying the flaw size from as low as $2 \mathrm{~nm}$ to $6 \mathrm{~mm}$ and the predictions corroborated well. It has been demonstrated that this approach has much potential for use in predicting the fracture toughness of ABCJ's having concerns of contamination. Nevertheless, RVE based 
approaches needs further investigations for an effective prediction of fracture toughness using multiple scales of undesirable bonding conditions.

\subsection{References}

[1] D. Taylor, "Applications of the Theory of Critical Distances to the Prediction of Crittle Fracture in Metals and Non-Metals," in ECF15, Stockholm, 2015.

[2] Z. Fang, B. Starly and W. Sun, "Computer-aided characterization for effective mechanical properties of porous tissue scaffolds," Journal of Computer-Aided Design, vol. 37, pp. 6572, 2005. 


\section{CONCLUSIONS}

The broad applicability of composite materials provides an avenue to replace existing metallic materials in aircraft structures. To maximize the efficiency of composites, the joining of structures need to be accomplished using adhesive bonding as opposed to conventional joining methods. An increased use in adhesively bonded composites will lead to significantly lighter structures. The difficulty in the acceptance of bonded structures centers around the validation of the service life of the bond i.e. its long term durability. Intrinsic factors such as contamination and their effects on bond performance needs to be significantly understood, before general acceptance in the aerospace community. Nevertheless, numerous research efforts have been initiated in aircraft community to improve their structural efficiency by leveraging adhesive bonding technologies.

As the strength of adhesively bonded joints continue to increase with improved materials and manufacturing processes, new applications for adhesively bonded composite joints evolve. As such, it is important to understand the additional factors from these applications that may influence bond performance. In this study, the influence of surface preparation on composite-adhesive bond quality was investigated through the development of a repeatable and scalable surface contamination procedure. Contaminant application and wetting characteristics were verified using FTIR, contact angle and surface free energy measurements. Both macroscale DCB testing and in situ micro scale ENF analysis showed a direct correlation between locations of contaminant application (macroscale) and the damage mechanims (microscale). Damage in the baseline specimen was confined to the crack tip region whereas the damage in the contaminated specimen initiated at the composite adhesive interface and propagated through the adhesive as loading increased. The increased compliance for contaminated specimens is due to the coalescence of microcracks that resulted in interlaminar failure. Validation of the contaminated and noncontaminated sites was verified using EDS results. In addition, the overall contaminated area played 
a major role in decreasing the bond quality, whereas the effects of application size (stamp size) were negligible.

The new test methodology developed in this study, can be an additional tool to the current quality management system for developing a reliable adhesively bonded structure. Pristine bonded joints and joints with undesirable bonding conditions were used to generate durability datasets that where conditioned at $95 \% \mathrm{RH}$ and $50^{\circ} \mathrm{C}$ for eight weeks. Results indicated that the length of exposure time was not significant enough to affect the bond performance. This information can assist future design engineers in setting test parameters for evaluating the long term durability of the joints. Conditioning for longer periods of time (80 weeks), did demonstrate some degradation in the bond performance due to absorption of moisture. The RVE unit cell approach adopted in this study, assists in predicting the fracture toughness of ABCJ's using undesirable bonding conditions. The developed model was successfully validated by varying flaw size from the nanometer to millimeter range and the predictions correlated well with the data obtained.

The overall results of this study can be used to improve the design of adhesively bonded composites and to evaluate the ability of NDE techniques to assess bond quality prior to implementation. For instance, hand-held FTIR scans can be used at various stages of composite processing/composite bonding to determine the presence of potential contaminant locations and overall affected area. Based on this study, standards for acceptable amounts of contamination can likely focus on the total contaminated area and not on the application size or the process adopted. In addition, future designs can incorporate direct evidence of the influence of weak bonds on overall performance in design and modeling software. This will lead to an enhanced confidence in predictions and a reduction in overall component weight and in the need for arbitrarily high safety factors or overdesigned mechanical fasteners. 


\section{VITA}

\section{VISHAL MUSARAMTHOTA}

EDUCATION AND EXPERIENCE

Aug 2011 - Present

Spring 2016

Aug 2011 - Dec 2015

Summer-Fall 2016

Aug 2011- Dec 2015

Fall 2014

2011-2013

Aug 2009 - May 2010

May 2008 - Aug 2009

Jan 2008 - May 2009

August 2003 - May 2007
Research Assistant, Applied Research Center, Miami, Florida, USA

Presidential Award in 2016 Outstanding Student Life Awards

Doctoral Candidate (Ph.D.) in Material Science and Engineering, Florida International University, Miami, Florida, USA

Dissertation Year Fellowship: University of Graduate School, Florida International University

Research Engineer, Advanced Biomaterials Laboratory, Miami, Florida, USA.

Doctoral Evidence Acquisition Fellowship: University of Graduate School, Florida International University

Secretary \& Treasurer, Material Advantage Chapter at FIU (Chapter won "Chapter of Excellence" all the years of service)

Materials Research Engineer, Plasma Forming Laboratory, Miami, Florida, USA

Graduate Research Assistant, Department of Mechanical and Materials Engineering, Miami, Florida, USA.

Master of Science (M.S.) in Material Science and Engineering, Florida International University, Miami, Florida, USA

Bachelor of Technology (B.Tech.) in Mechanical Engineering, PRRM College of Engineering and Technology, Hyderabad, India

Student Government Association Travel Grant Scholarships to present a research work at various national and international conferences. 


\section{PUBLICATIONS AND PRESENTATIONS}

1. Ping Liang, Rakesh Guduru, Ali Hadjikhani, Emmanuel Stimphil, Vishal Musaramthota, Alexander Franco Hernandez and Sakrat Khizroev, "Online Control of Rechargeable Batteries Using Multiferroic Nanoparticles”, Scientific Reports, Nature. (under review)

2. Vishal Musaramthota, Dwayne McDaniel, Benjamin Boesl and Norman Munroe, "Multi-Scale Fracture of Adhesively Bonded Composite Joints with Undesirable Bonding Conditions", Composites Part B: Engineering. (submitted)

3. Vishal Musaramthota, Dwayne McDaniel, Tomas Pribanic, Benjamin Boesl and Norman Munroe, “Assessing Durability for Adhesively Bonded Composite Joints using Undesirable Bonding Conditions”. (manuscript under preparation)

4. Norman Munroe, Vishal Musaramthota, Jose Gonzalvez and Kinzy Jones, "Influence on Material Composition in Modular Hip Prostheses", SurFACTS in Biomaterials, Volume 20, Issue 2, Spring 2016.

5. Puneet Gill, Vishal Musaramthota, Norman Munroe, Amit Datye, Rupak Dua, Waseem Haider, Anthony McGoron and Ryszard Rokicki, "Surface modification of Ni-Ti alloys for stent application after magnetoelectropolishing”, Materials Science and Engineering C, 50 (2015) 37-44.

6. Paola Sepulveda-Medina, Yelena Katsenovich, Vishal Musaramthota, Michelle Lee, Brady Lee, Rupak Dua and Leonel Lagos, "The Effect of Uranium on the Bacterial Viability and Cell Surface Morphology Using Atomic Force Microscopy in the Presence of Bicarbonate Ions”, Journal of Research in Microbiology, 166 (5), 2015, pp 419-427.

7. Vishal Musaramthota, Sushma Amruthaluri, Amit Datye and Norman Munroe, "Dual Scale Microstructure and Surface Analysis of Ti-Mo-Zr-Fe and Ti-Mo-Nb-Fe alloys for Orthopedic Implants”, proceedings at 29th Southern Biomedical Engineering Conference, Miami, May 13, 2013.

8. Vishal Musaramthota, Tomas Pribanic, Dwayne McDaniel, Norman Munroe, Xiangyang Zhou, Juanjuan Zhou and Sirui Cai, "A Study on the Contamination Effects and Durability Assessment of Adhesively Bonded Composite Joints”, International SAMPE Symposium and Exhibition (Proceedings), Wichita, Kansas, Oct 22-24, 2013.

9. V. Musaramthota, T. Pribanic, D. McDaniel, X. Zhou, J. Zhou and Z. Wang, S. Cai, "Effect of Surface Contamination on Composite Bond Integrity and Durability", Proceedings of the 2013 Joint Advanced Materials Structures Center of Excellence 9th Annual Technical Meeting, Seattle, WA, April 9-10, 2013.

10. Srinivasa R. Bakshi, V. Musaramthota, D. Lahiri, V. Singh, S. Seal and A. Agarwal. "Spark Plasma Sintered Tantalum Carbide: Effect of Pressure and nano-Boron Carbide Addition on Microstructure and Mechanical Properties”, Materials Science and Engineering A, 528 (2011) 1287-1295.

11. Srinivasa R Bakshi, Vishal Musaramthota, David.A. Virzi, Anup K. Keshri, Debrupa Lahiri, Virendra Singh, Sudipta Seal and Arvind Agarwal,“Carbon Nanotube Reinforced Tantalum Carbide Composites by Spark Plasma Sintering: Effect of pressure, Carbon Nanotube Length and Dispersion Technique,” Materials Science and Engineering A, 528 (2011) 2538-2547.

12. Vishal Musaramthota, Dwayne McDaniel and Benjamin Boesl, "Fracture Behavior on Adhesively Bonded Composite Joints with Undesirable Bonding Conditions: A Multiscale Approach”, International SAMPE Symposium and Exhibition (Proceedings), Long Beach, CA, May 23-26, 2016.

13. Dwayne McDaniel, Vishal Musaramthota, Benjamin Boesl and Xiangyang Zhou, "Effect of Surface Contamination on Composite Bond Integrity and Durability", Advanced Materials in Transport Aircraft Structures (AMTAS 2015), Seattle, Washington, November 04, 2015. 